\title{
NOVEL METHODOLOGY FOR SAFETY AND ECONOMIC EVALUATION OF RED-LIGHT CAMERAS AT SIGNALIZED INTERSECTIONS
}

\author{
by \\ Amal Abdulsalam \\ A thesis submitted to the Faculty of Graduate and Postdoctoral Affairs \\ in partial fulfillment of the requirements for the degree of \\ Doctor of Philosophy \\ in \\ Civil and Environmental Engineering \\ Carleton University \\ Ottawa, Ontario
}

The Doctor of Philosophy in Civil and Environmental Engineering Program is a joint program with the University of Ottawa, administered by the Ottawa Carleton Institute for Civil Engineering

(C) 2017, Amal Abdulsalam 


\begin{abstract}
Red-light running is a phenomenon that has led to frequent collisions causing fatalities, injuries, and property damage. Red-light cameras (RLCs) have been used in an attempt to reduce the frequency of these collisions. The main objectives achieved in this thesis are: (1) The site-selection bias of traditional methodologies were corrected by using a novel methodology (propensity score matching); (2) The fixed and random-effect panel regressions were utilized to account for the spatial and temporal correlations in the data; (3) The identification of how RLC effectiveness varies by site characteristics through the use of interaction terms; (4) The determination of spillover distances and times resulting from the presence of RLCs; (5) The benefit-cost methodology supported by economic analysis and sensitivity analysis using the novel methodology; and (6) The establishment of guidelines for effective implementation of the RLC treatment.

The research used field data from the City of Ottawa (Canada) involving 34 RLC intersections and 14 control intersections observed for the period 1999-2012. The results from this thesis indicate a consistent significant reduction in angle and turning movement collisions of $19 \%$ and $21 \%$, respectively, and an increase in rear-end and sideswipe collisions of $12 \%$ and $6.7 \%$, respectively. The magnitude and the direction of these effects are comparable to results of previous studies in the literature. The benefit-cost analysis, based on social costs of collisions avoided, the spillover effects and fine revenue, yielded an overall annual cost savings to the community of over $\$ 4.4$ million CAD across the 34 RLC intersections; with a benefit-cost ratio of 4.50. An extended cost sensitivity analysis was incorporated to quantify the robustness of the base-case conclusions.
\end{abstract}


RLC implementation decisions represent a major policy action, with serious repercussions for public safety. This thesis enhances such decisions by establishing effective implementation guidelines using rigorous, novel statistical analysis methods aiming to assist in this key policy effort. Furthermore, this thesis contributes to quantifying the RLC spillover effects as well as identifying critical road factors that the RLC treatment is likely to affect and benefit from. These contributions are all critical components of the traffic safety management process. 


\section{Acknowledgements}

First and foremost, I would like to express my deepest gratitude to my supervisors Professors Said Easa, A.O. Abd El Halim and Dane Rowlands, for their continuous encouragement, support and optimism. My sincere thanks and appreciation to all three of them for being part of this journey, making this thesis possible and for always helping me keep things in perspective.

A special thanks to Professors Yasser Hassan and Dan Patterson for being an inspiration to my thesis topic and for their support throughout this journey. I also want to express my gratitude to all my colleagues and friends in the department of Civil and Environmental Engineering and outside the department for sharing their expertise and experiences, and for providing valuable feedback throughout this research. I would also like to thank Carleton University for providing the working environment for my research study. Also, a very special gratitude goes out to my workplace in Kuwait, Pan Arab Consulting Engineers, for their support and understanding in allowing me to pursue higher levels of education and knowledge.

This thesis could not have been completed without the collaboration and cooperation of the people who provided data from localities; staff at Customer and Traffic Information Services at the City of Ottawa along with Mr. Stuart A. Edison and his team at Traffic Engineering \& Control, Traffic Operations, City of Ottawa. This research is financially supported in part by a Discovery Grant from the Natural Sciences and Engineering Research Council of Canada (NSERC) and by Carleton University.

Finally, my deep and sincere gratitude goes out to my entire family in Kuwait for their continuous and unparalleled love, help and support. I am forever indebted to my 
parents for giving me the opportunities and experiences that have made me who I am. This journey would not have been possible if not for them, and I dedicate this milestone to them. 


\section{Table of Contents}

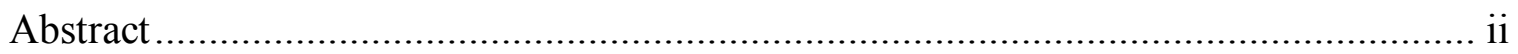

Acknowledgements …………………............................................................... iv

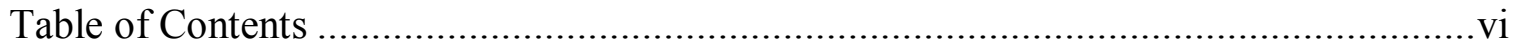

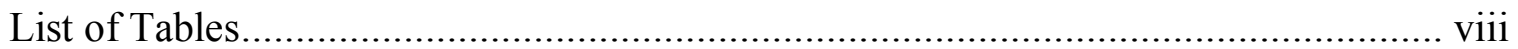

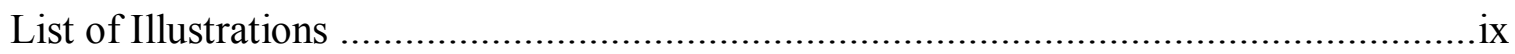

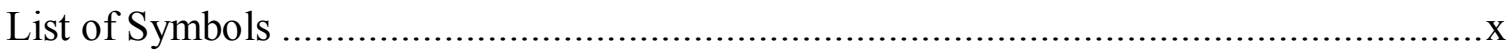

List of Appendices ..................................................................................................

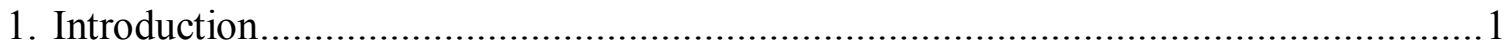

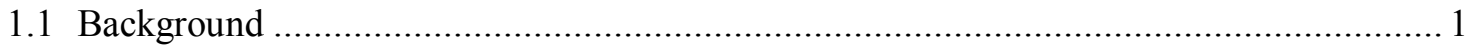

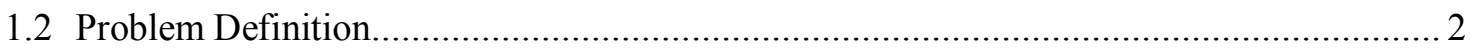

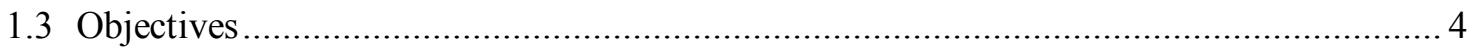

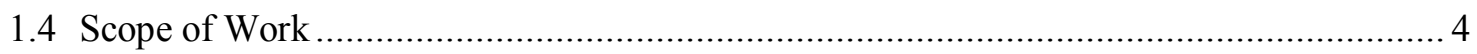

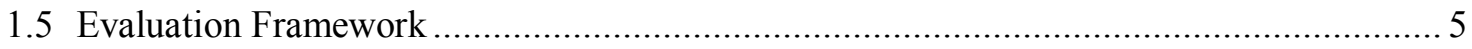

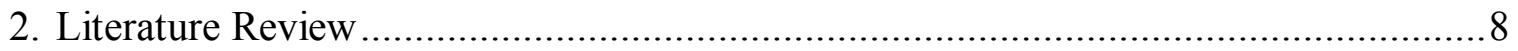

2.1 Safety at Signalized Intersections ……………………...................................... 8

2.2 RLC Enforcement Against RLR ………………………..................................... 12

2.2.1 Impact of RLC Programs from Previous Research...…………………………………. 14

2.2.2 Spillover Effect and Collision Migration …………………………………………..... 23

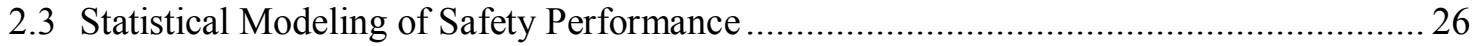

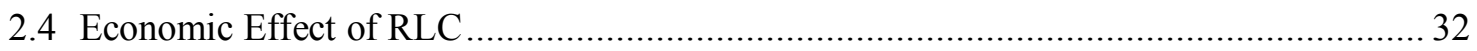

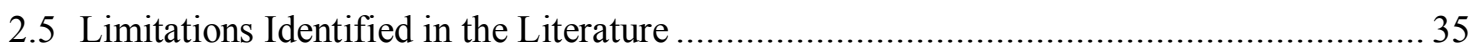

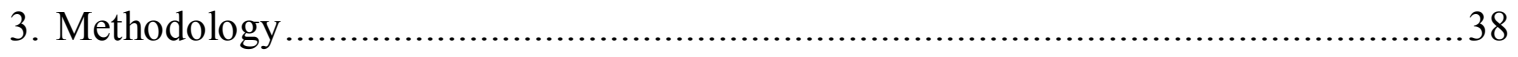

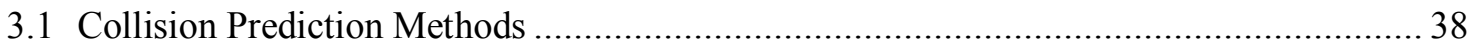

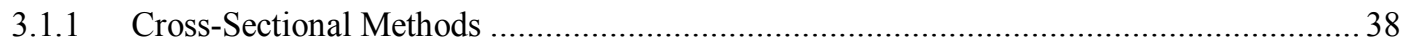

3.1.2 Empirical Bayes Method ............................................................................ 43

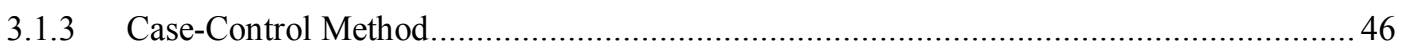

3.1.4 Incorporating Interaction Terms ................................................................................ 50

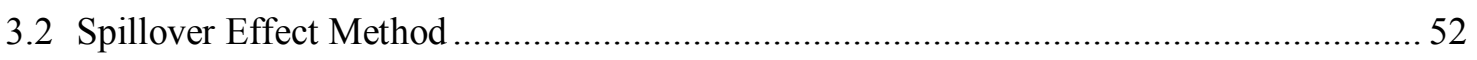

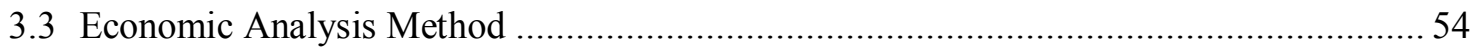

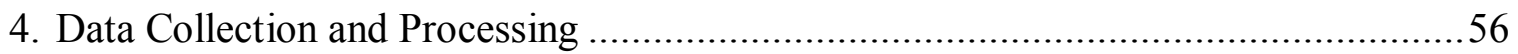

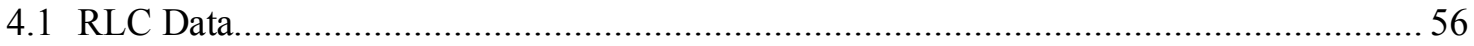




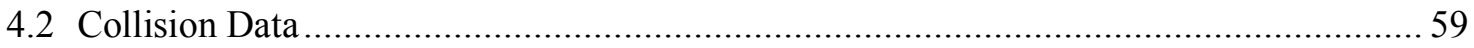

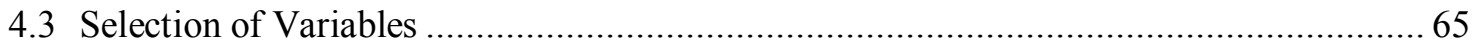

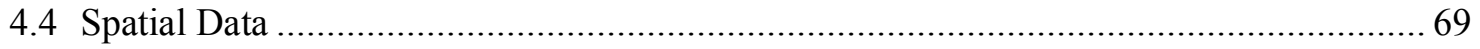

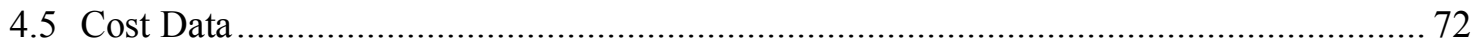

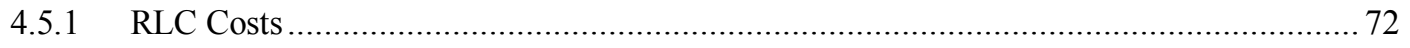

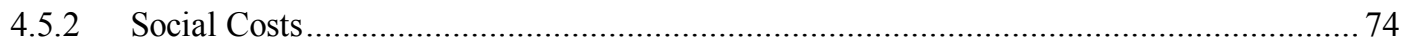

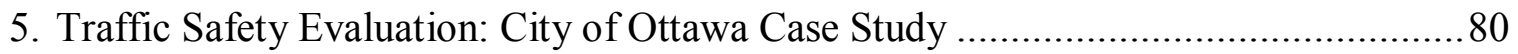

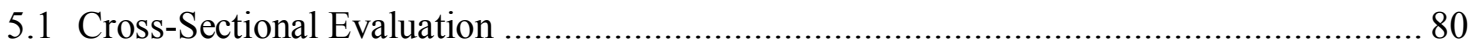

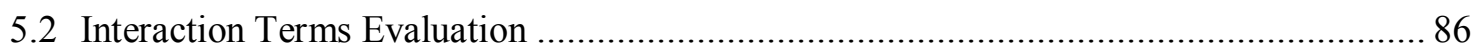

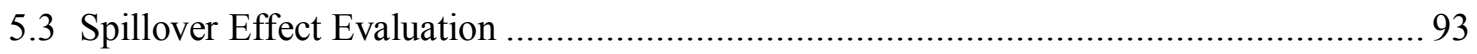

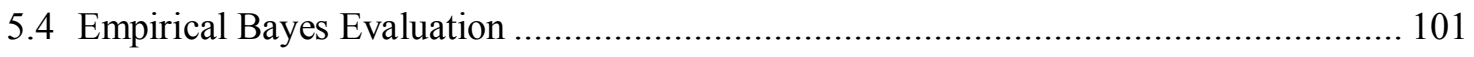

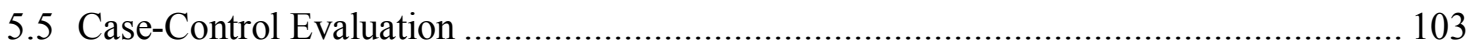

5.6 Comparison of Results from the Statistical Methods ............................................... 109

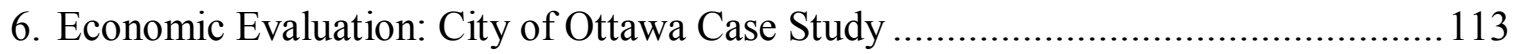

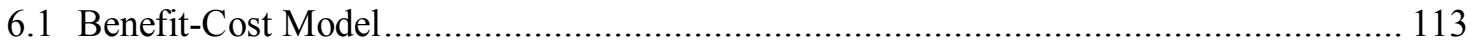

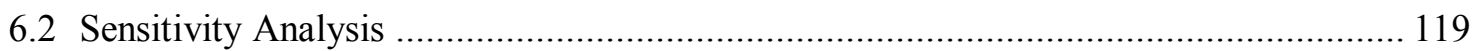

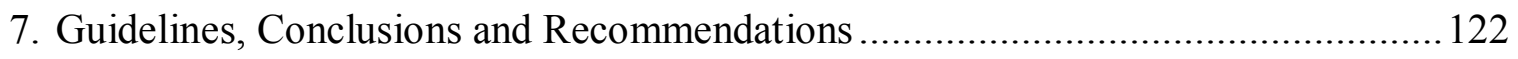

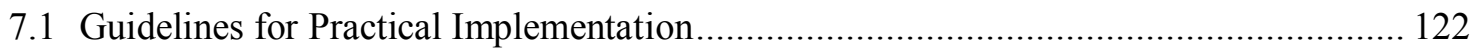

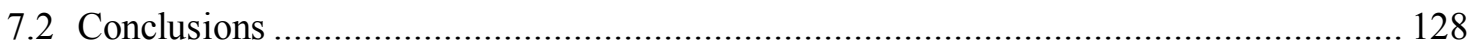

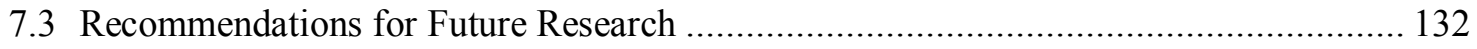

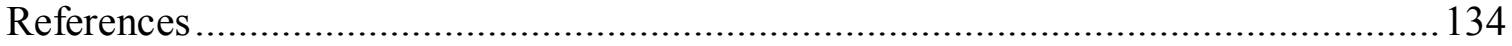




\section{List of Tables}

Table 2.1 - Ottawa's RLC site selection criteria and process (Adapted from Table 2 of Leclair, $2004)$

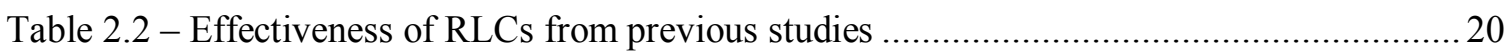

Table 2.3 - Methods used to analyze observational data in traffic safety .................................2

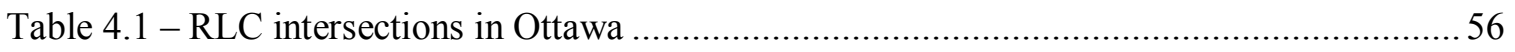

Table 4.2 - Summary of collision database by classification and impact type for RLC and non-

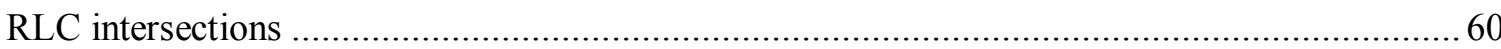

Table 4.3 - Average number of collisions (per intersection per year) by classification and type for

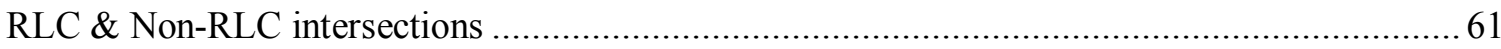

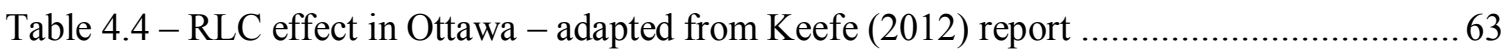

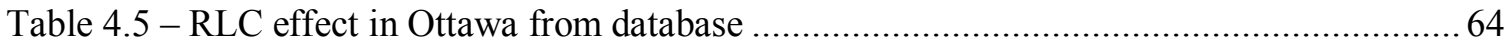

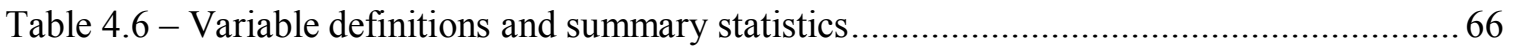

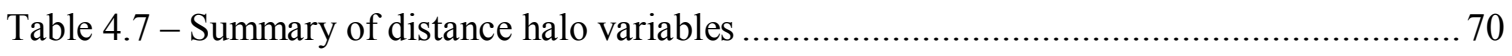

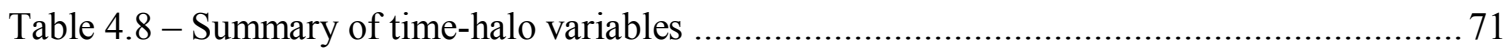

Table 4.9 - Estimated net revenue (from fines) of the RLC program in Ottawa .......................... 74

Table 4.10 - Ontario's estimated social costs of traffic collisions ................................................ 76

Table 4.11 -Top 3 RLC intersections that benefited after the RLC installation ........................... 77

Table 4.12 -Top 3 RLC intersections that hindered after the RLC installation .......................... 77

Table 5.1 - NB regression results based on best fit models (IRR coefficients) ........................... 82

Table 5.2 - FE and RE NB regression results for RLC effect .............................................. 84

Table 5.3 - Marginal effect of the RLC covariate (xtnbreg* estimates) .................................... 87

Table 5.4 - Impact of RLC in terms of percentage of collision reduction (xtnbreg) .................... 88

Table 5.5 - Significant interactions of RLC with different covariates according to collision type

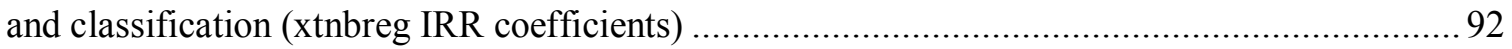

Table 5.6 - Marginal effect of the RLC covariate (nbreg* estimates) ...................................... 94

Table 5.7 - Impact of RLC in terms of percentage of collision reduction (nbreg) ...................... 95

Table 5.8 - Best fit regression models with time and distance halo variables (IRR coefficients) 96

Table 5.9 - Significant interactions from spillover models (nbreg) ....................................... 100

Table 5.10 - SPF models (calculated from full set of non-RLC intersections) .......................... 101

Table 5.11 - Expected number of collisions from the calculated SPFs .................................. 102

Table 5.12 - Impact of RLC (ATT) from PSM method on different collision impact types ...... 107

Table 5.13 - Impact of RLC (PSM ATT) in terms of percentage of collision reduction............ 109

Table 5.14 - Summary of the results from the different statistical approaches .......................... 109

Table 5.15 - What the different statistical methods adjust for .............................................. 111

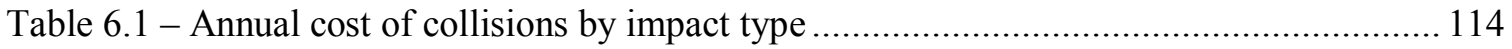

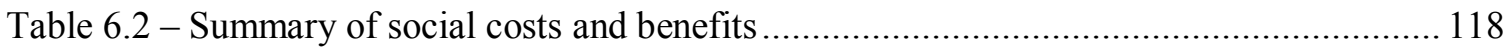

Table 6.3 - Annual monetary benefits associated with the RLC treatment for each of the five

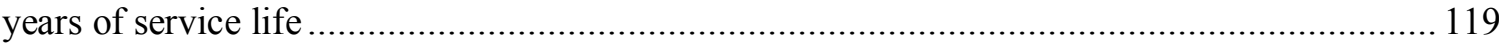

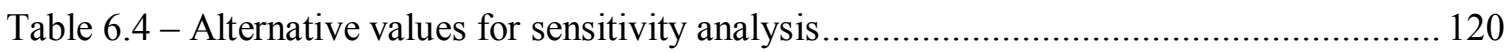

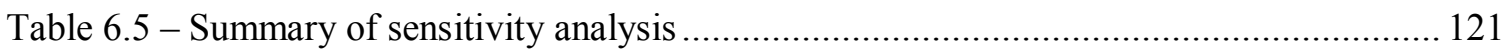




\section{List of Illustrations}

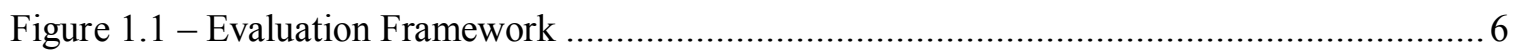

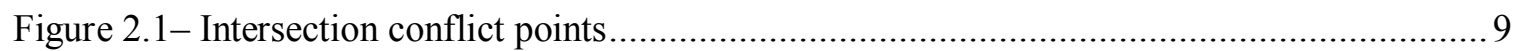

Figure 2.2 - Common collision types at signalized intersections ........................................ 9

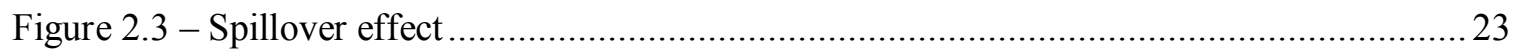

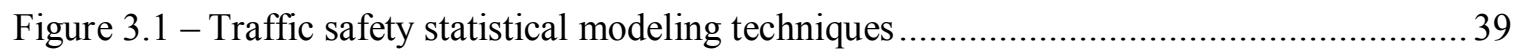

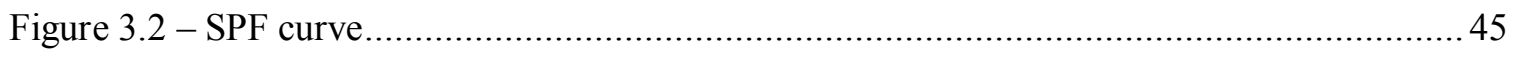

Figure 3.3 - Identifying the three closest facilities to each incident ....................................... 53

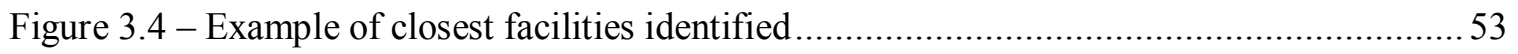

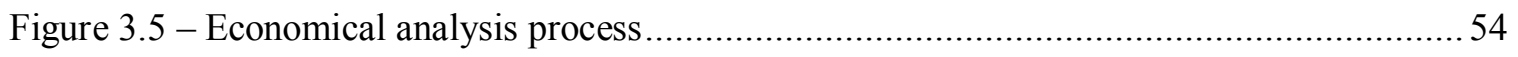

Figure 4.1 - Location of the 34 RLC intersections and comparison intersections in Ottawa .......57

Figure 4.2 - A typical RLC set up in Ottawa (photos by: Amal Abdulsalam) ...........................58

Figure 4.3 - A typical warning sign of an approaching RLC intersection (photo by: Amal

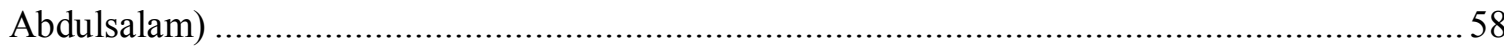

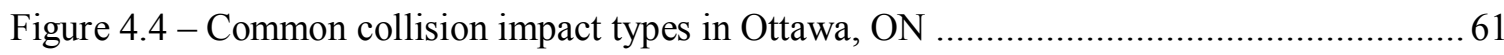

Figure 4.5 - RLC intersections with fatal collisions ........................................................... 79

Figure 5.1 - Significant interaction terms for total collisions ............................................ 90

Figure 5.2 - Non-significant interaction terms for total collisions ......................................... 91

Figure 5.3 - Distribution and matching of the propensity scores estimation between RLC and

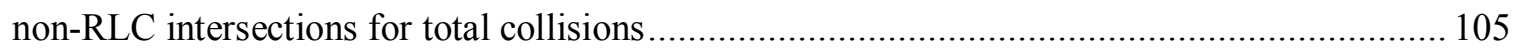

Figure 5.4 - Percent standardized bias of covariates before and after matching ....................... 106 


\section{List of Symbols}

$\beta_{i}=$ Coefficients quantifying the relationship between $X$ and $\lambda$

$\varepsilon=$ Random error term

$\lambda=$ Expected number of collisions per year on a roadway segment

$\tau=$ Impact of treatment

$\Gamma=\mathrm{Gamma}$

$\omega=$ Intercept

AADT $=$ Average annual daily traffic

$\mathrm{ATE}=$ Average treatment effect

ATT $=$ Average treatment effect on the treated

$\mathrm{BCR}=$ Benefit-cost ratio

$D=$ Indicator of exposure to treatment

$E\left(y_{i}\right)=$ Expected collision frequency at location $i$

$\mathrm{EB}=$ Empirical Bayes

$\mathrm{FE}=$ Fixed-effects

FENB $=$ Fixed-effects negative binomial

GIS $=$ Geographic information system

IPWRA = Inverse-probability-weighted regression adjustment

$L_{i}=$ A nominal (dummy) variable

$\mathrm{MTO}=$ Ministry of Transportation Ontario

$\mathrm{NB}=$ Negative binomial

$\mathrm{NPV}=$ Net present value

ZINB $=$ Zero-inflated negative binomial

$\widehat{p}=$ Propensity score

$\mathrm{PSM}=$ Propensity score matching

$\mathrm{RE}=$ Random-effects

RENB $=$ Random-effects negative binomial

$\mathrm{RLC}=$ Red-light camera

$\mathrm{RLR}=$ Red-light running

RTM $=$ Regression to mean

$\mathrm{SPF}=$ Safety performance function

$x_{\mathrm{i}}=$ Explanatory variable

$Y_{1 i}=$ Potential outcome in case of treatment

$Y_{0 i}=$ The potential outcome in absence of treatment

ZINB $=$ Zero-inflated negative binomial

$\mathrm{ZIP}=$ Zero-inflated Poisson 


\section{List of Appendices}

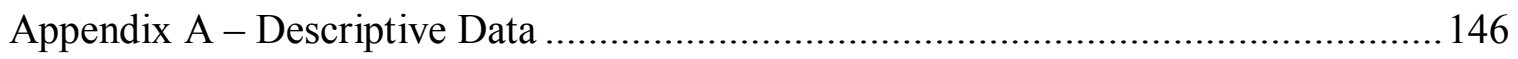

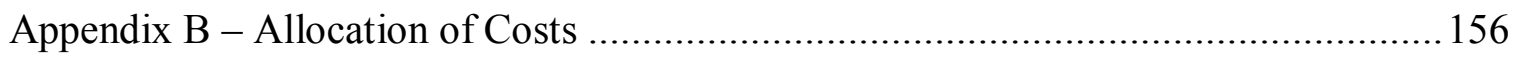

Appendix C - Histogram of collision frequency by collision impact type .................... 157

Appendix D - Means and Variances ....................................................................... 160

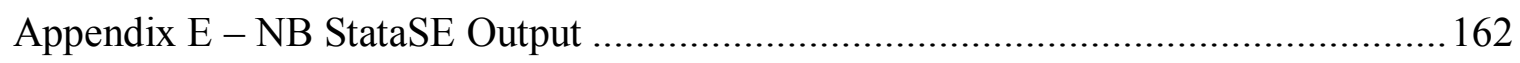

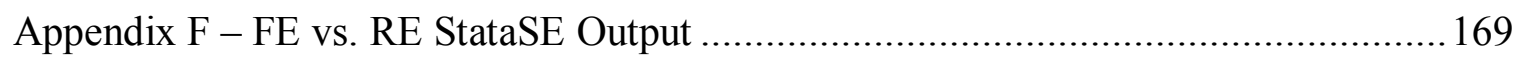

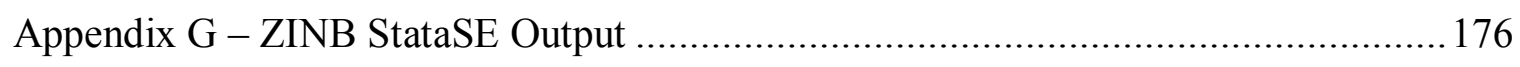

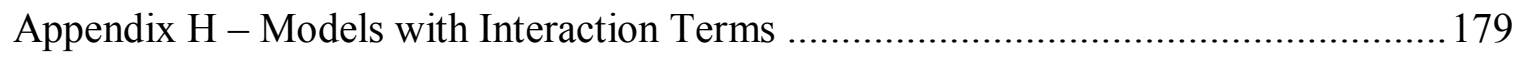

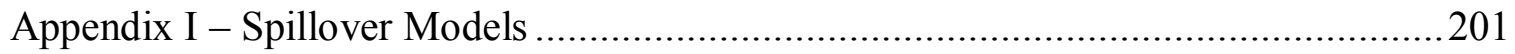

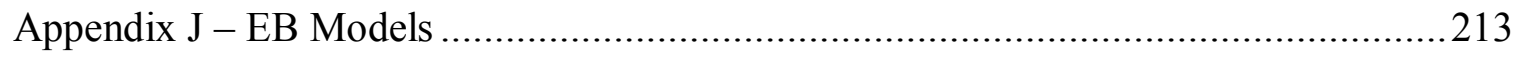

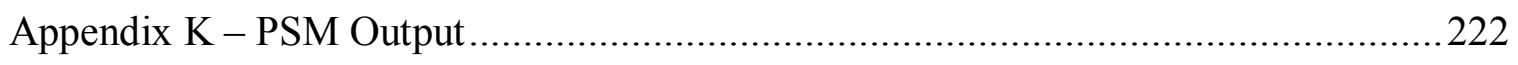

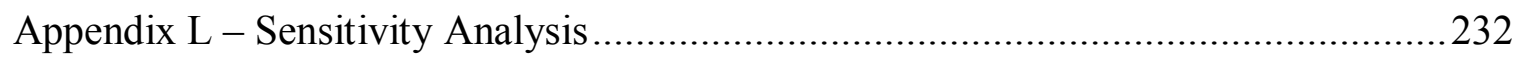




\section{Introduction}

\subsection{Background}

Traffic signals are intended to promote safe and efficient traffic flow at busy intersections. However, the level of safety achieved largely depends on drivers' compliance with the traffic signals. Red-light running (RLR) is a behavioural phenomenon that occurs when a motorist enters an intersection after the traffic signal light has turned red (FHWA, 2009). Research shows that many drivers routinely violate the red-light signals, placing themselves and other road users at risk for serious collisions (Retting et al., 2007). In 2009, RLR caused $30 \%$ of the fatalities and $40 \%$ of the serious injuries at signalized intersections across Canada (Transport Canada, 2011). The problem of RLR is not limited to Canada, it is a worldwide phenomenon. In the United States, RLR resulted in 714 fatalities and an estimated 144,000 injuries in 2011 (IIHS, 2013a,b).

The social costs caused by traffic collisions are massive, and include property damage, insurance claims, hospital and health care costs, first responder costs (i.e. police, fire, and ambulance services) and traffic delays. The social cost of motor vehicle collisions in Canada in 2004 was estimated to be $\$ 63$ billion (Vodden et al., 2007). According to the Ministry of Transportation Ontario (2007), fatal collisions account for less than $1 \%$ of reportable collisions in Ontario in 2004, but they account for 64\% ( $\$ 11$ billion) of the total social cost.

Implementing an effective safety countermeasure is critical in the overall safety management process. Several factors are considered when selecting a countermeasure, including the cost and its effectiveness in reducing specific collision types (e.g. rear-end, angle, night time, etc.) or severities. One of the key enforcement initiatives which has 
been used increasingly over the past decade to assist and facilitate enforcement against RLR is red-light cameras (RLCs). A RLC is a type of traffic enforcement camera that captures an image of a vehicle which has entered an intersection in spite of a red-signal phase. Camera enforcement is intended to modify driver behaviour through both general deterrence and punishment of individual violators. Determining the effectiveness of a countermeasure is based on an accurate assessment of the cause-effect relationship between the countermeasure and the outcome. Therefore, it is important to: (1) utilize appropriate statistical methods, (2) identify factors that contribute to the collision occurrence and (3) assess the effectiveness of the countermeasure in preventing collisions.

\subsection{Problem Definition}

Important data and methodological issues have been identified in the literature regarding countermeasure effectiveness assessment. Studies have utilized different methodologies, different populations and different data sources which may lead to flawed collision frequency predictions and incorrect inferences relating to the factors that determine the frequency of collisions. Conducting a simple before-after study to assess effectiveness has an underlying limitation in that it is not possible to distinguish the regional effects from the intervention. Another limitation is failure to account for regression to the mean (RTM). RTM occurs when the extreme cases chosen in one period are likely to experience lower collision frequencies in the next period, and vice versa, because of random variation (FHWA, 2011). The presence of RTM can exaggerate the positive effects of a countermeasure. Issues of selecting control groups, which can be used to 
address the RTM phenomena, and how the treatment and control groups are matched are not clearly outlined in the literature.

At the present time, there is a lack of clear guidelines to use when evaluating the decision to install RLCs at specific intersections. A series of issues related to RLCs addressed in this research have not been investigated in depth before, such as how effectiveness varies by site characteristics and under what conditions RLCs perform most effectively. The absence of important interactions of control variables can affect the accuracy and interpretations of estimations. In addition, ignoring possible spillover effects from the RLC to intersections without the countermeasure can lead to an underestimation of the true benefits. A comparison of results from the different statistical safety evaluation methods is needed in order to establish a accurate estimations for assessing and evaluating the regional conditions necessary to make proper decisions on using RLCs as a safety countermeasure.

There is a need to analyze how the intersection characteristics influence the effects of RLCs, and identify the locations which have the most benefit from treatment. Most previous studies on RLCs usually report an average treatment effect, which neglects the fact that the effects of RLCs may differ systematically by intersection characteristics. It is desirable that the treatment is implemented in a way that maximises effectiveness. By revealing patterns of the RLC treatment effect, policy makers can assign RLC treatments to intersections most likely to benefit from the treatment, so as to improve the costeffectiveness of intervention.

In light of these limitations, this thesis contributes to the field by developing a reliable assessment methodology using novel statistical methods to estimate the treatment 
effect of RLCs at signalized intersections on road safety to help set guidelines for practical implementation.

\subsection{Objectives}

The main objectives of this thesis are: (1) to correct for the site-selection bias of traditional methodologies by using a novel methodology (propensity score matching); (2) to use the fixed-effect and random-effect panel regressions to account for the spatial and temporal correlations in the data; (3) to identify how RLC effectiveness varies by site characteristics by using interaction terms; (4) to measure spillover distances and times resulting from the presence of RLCs; (5) to apply benefit-cost methodology supported by economic analysis and sensitivity analysis using these new estimation methods; and (6) to establish guidelines for effective implementation of the RLC treatment.

RLC implementation decisions represent a major policy action, with serious repercussions for public safety. This thesis enhances such decisions by using rigorous statistical analysis to assist in this key policy effort. This thesis will contribute to more accurate estimation of the direct effectiveness of RLCs, quantifying their spillover effects, and identifying critical road factors which the RLC treatment is likely to have the largest effect on and would benefit the most. These contributions are all critical components of the traffic safety management process.

\subsection{Scope of Work}

The framework developed in this thesis addresses evaluation of safety and economic aspects of RLCs. To support this evaluation, collision data were collected from the City of Ottawa in Ontario, Canada for 34 signalized intersections as part of the RLC treatment group and for 14 non-RLC signalized intersections as part of the control group. The study 
duration involved collision data from 1999 to 2013. Examining site selection criteria and its effects on safety was beyond the scope of this thesis. This thesis analyzes vehicle collisions only, pedestrian and cyclist collisions are beyond the scope of this research. The effectiveness of the RLCs were analysed in terms of reduction in the frequency of reported collisions by impact type and their associated collision costs by classification (severity). This thesis will not provide information on evaluating the effect of RLCs on RLR violation reduction due to the lack of detailed violations data from City of Ottawa localities.

\subsection{Evaluation Framework}

An evaluation framework was developed to guide the work towards achieving the objectives of this thesis. Figure 1.1 illustrates the different tasks of the framework. The first step was to conduct a comprehensive literature review to study safety at signalized intersections and the different evaluation methods conducted in assessing the effectiveness of the RLC programs. Based on the inconsistencies and deficiencies found in the literature review, the motivation and objectives of this thesis were established. Subsequently, data and information have been collected to develop a statistically based framework consisting of two streams: traffic safety and economic assessment.

As part of the analytical evaluation of the traffic safety assessment, data for control variables were obtained from different sources and aggregated at the same level to explore the various factors affecting the collisions and the RLC intervention. Some of the various factors collected included: a collision database, traffic characteristics, geometric characteristics, and cost data. 
- Intersection safety

- Impact of RLC programs

- Statistical techniques for RLC safety evaluation

- Economic effect of RLC

- Identify limitations in the literature

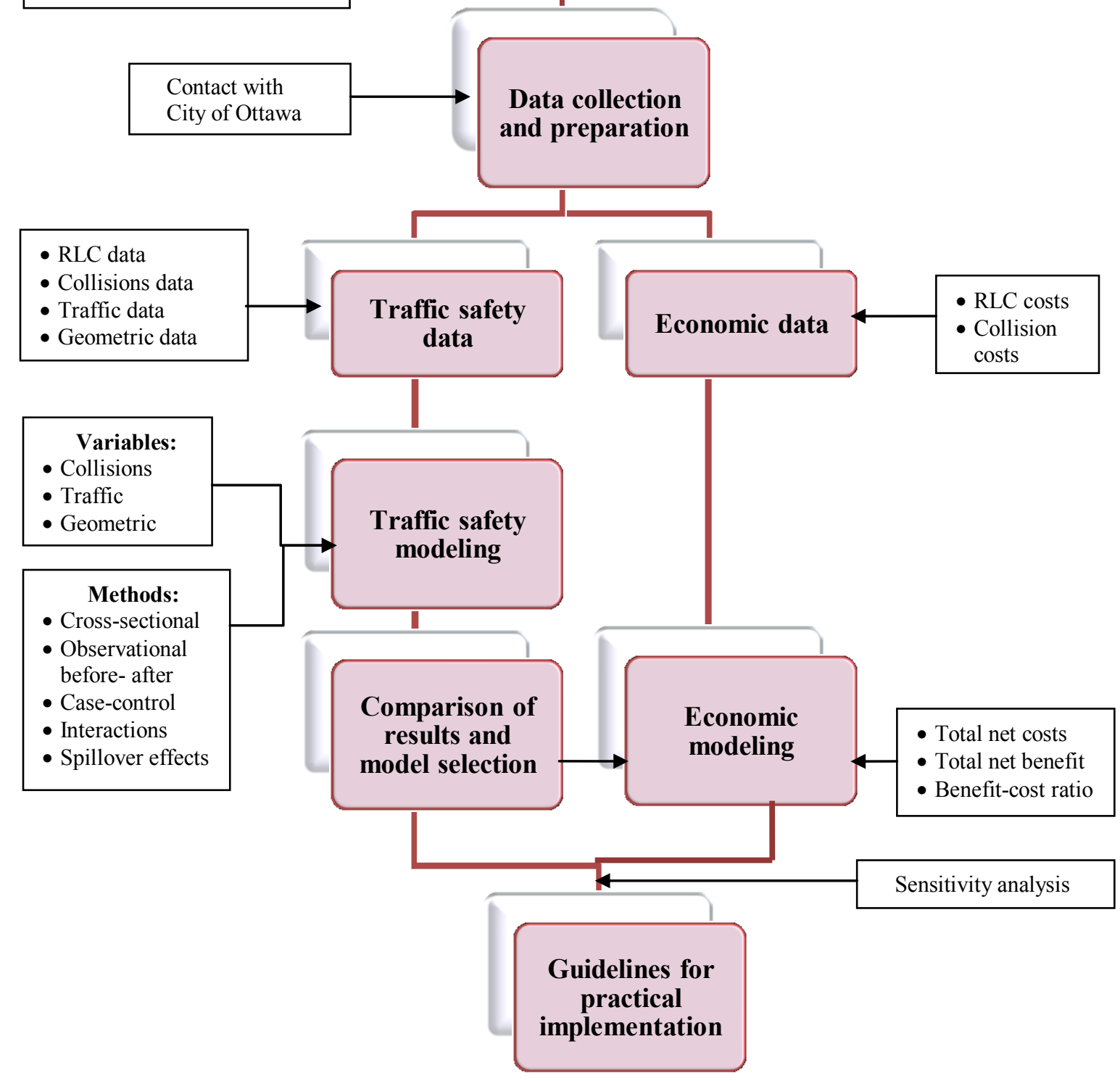

Figure 1.1 - Evaluation Framework 
Next, observational analytical methods were utilized to examine the causal relationship between traffic interventions and traffic collisions. The three statistical observational methods applied were: cross-sectional studies, observational before-andafter studies, and case-control studies.

Results of the different evaluation methods were compared in order to evaluate the difference in the results when assessing the effectiveness of the RLC intervention. This comparison is done by quantifying the expected number of collisions has the intervention not been applied and comparing these results to the observed number of collisions in the post-intervention period. The number of collisions avoided were used in the economic evaluation.

The economic evaluation involved collecting data and information relevant to RLCs costs and social costs of collisions to assess the costs and performance of the RLC treatment. A monetary value was assigned to the cost of a collision based on its classification and a comparison between the expected number of collisions had the treatment not been implemented (i.e. derived from the statistical methods). The observed number of collisions that actually occurred with the treatment implemented provided the basis for the benefit-cost analysis of safety effectiveness. The purpose of the benefit-cost analysis was to determine the net societal benefits of the RLC intervention through an assessment of all benefits and costs associated with the operation of RLCs.

The ultimate goal was to provide a better understanding of the causal effects of the RLC intervention on road safety by utilizing appropriate evaluation methods. This led to developing guidelines for policy-makers when evaluating the safety outcomes of RLCs and hence improving the prevention of RLR collisions. 


\section{Literature Review}

A comprehensive literature review was carried out to study research work performed in the field of RLC assessment related to the objectives of this thesis. The studies reviewed in this chapter covered safety at signalized intersections, enforcement against RLR, the impact of RLC programs, collision prediction modeling techniques, and economic effects of RLCs. The primary sources of literature reviewed were Accident Analysis and Prevention publications, Transport Canada reports, Traffic Injury Prevention publications, Institute of Transportation Engineers' publications, conference proceedings of the Transportation Research Board, the National Center for Biotechnology Information and City of Ottawa reports. This chapter is intended to highlight the latest available statistical methods used in treatment assessment and to identify the gaps and limitations in the literature related to the RLC safety assessment.

\subsection{Safety at Signalized Intersections}

Traffic signals are used to assign the right-of-way to vehicles passing through intersections so conflicting movements do not occur. There are 32 conflict points on a 4legged intersection, as shown in Figure 2.1. Conflict points are locations in the mid-block or on the approaches to an intersection where vehicle paths merge, diverge, or cross and can lead to collisions.

While installing traffic signals at intersections can often improve safety and traffic flow, such intersections are often studied because they are the scenes of many devastating collisions. Some drivers run red-lights at intersections knowing that there is an all-red light phase before the new green light appears, and other drivers jump the green light or complete a left-turn on the yellow light, resulting in an intersection collision, often at 


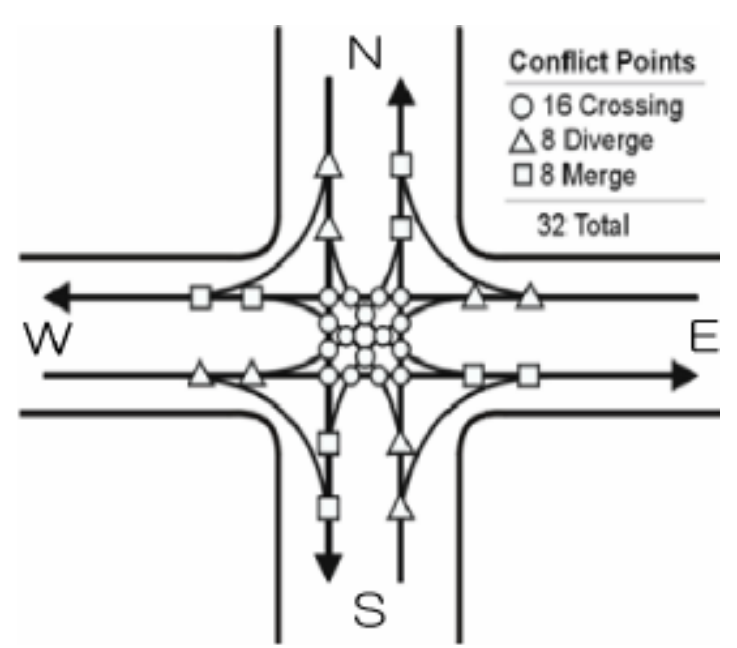

Figure 2.1- Intersection conflict points

high speed (Transport Canada, 2011). Figure 2.2 displays a visualization of some of the more commonly discussed collision impact types at signalized intersections due to their repeated occurrences and severities; angle collisions, turning movement collisions, rearend collisions and sideswipe collisions.

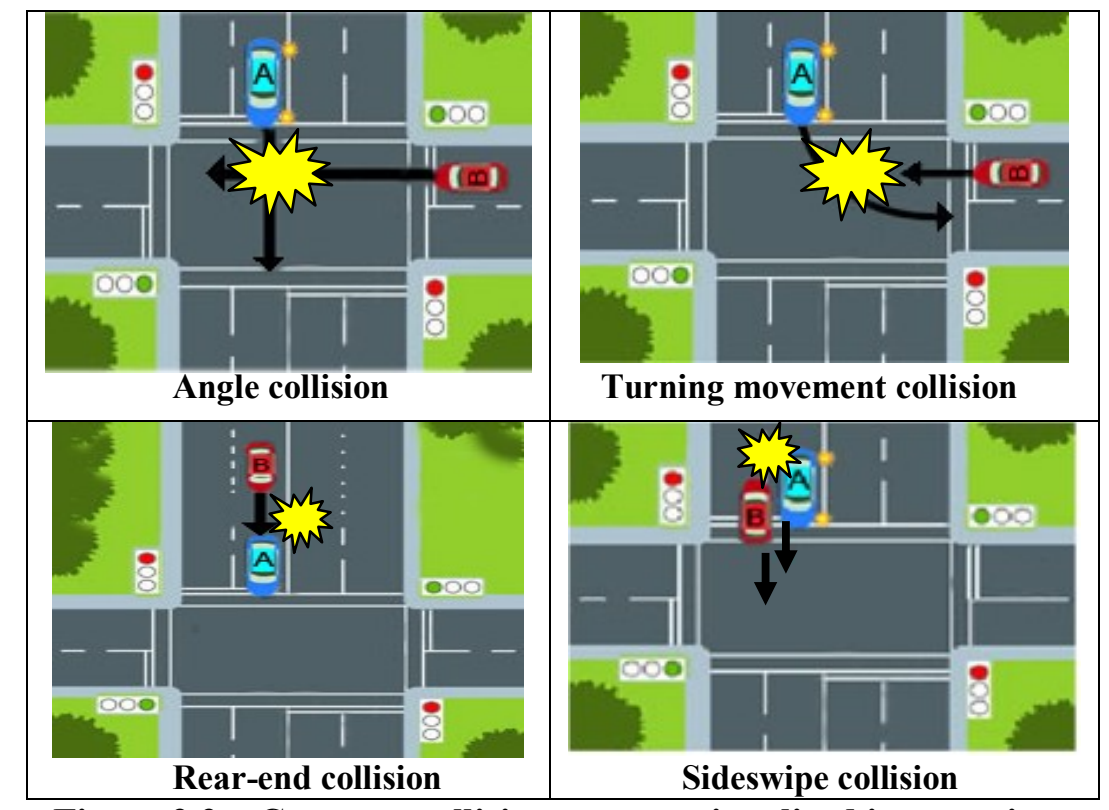

Figure 2.2 - Common collision types at signalized intersections

Angle collisions at signalized intersections are seen as most relevant to RLR. They involve two vehicles traveling in perpendicular directions (two adjacent approaches) that collide with each other. Since traffic signals are intended to separate 
conflicting movements, one of the vehicles involved in the collision would have had to have proceeded through on a red-signal. The frequency of angle collisions is therefore an indicator of a RLR problem occurring at an intersection. In 2014, there were 655 reported angle collisions at signalized intersections in Ottawa (City of Ottawa, 2016).

A turning movement collision occurs when a left-turning vehicle collides with an on-coming vehicle from the opposite direction; either vehicle may be the red-light violator. A RLR related collision may also occur when a left-turning vehicle strikes a vehicle proceeding through the intersection on an adjacent approach.

Rear-end collisions occur when a vehicle following the vehicle ahead collides into the back of the vehicle. Rear-end collisions are not the direct result of RLR but rather the result of vehicles stopping for a red-signal at an intersection while others behind them do not. By taking action to avoid a rear-end collision, a vehicle may sideswipe a second, adjacent vehicle.

Many sideswipe collisions happen when a driver overreacts to a road hazard. The initial sideswiping contact is normally just the beginning of a more serious vehicle collision. Most people assume that a sideswipe collision usually results in a minor collision involving minor damages to the vehicles and little to no physical injuries. However, they can lead to loss of vehicle control leading to a rollover or other potentially tragic results including severe physical injuries and even death. Seeing the different nature of collision impact types, it is more valuable to focus on target collisions than simply examining the total collisions when conducting causal analysis.

A report published by City of Ottawa (2012), identified that the peak times for angle, turning movement and rear-end collisions are all generally during daylight. 
Another important finding is that the peak collisions for these three collision impact types all happen during clear weather conditions. Therefore, bad weather conditions and night time does not seem to be critical factors triggering collisions.

As for collision classifications, a study conducted by the Ministry of Transportation Ontario (2011) found that $19 \%$ of fatal collisions and $28 \%$ of personal injury collisions occur at intersections. Disobeying the traffic signal is involved in $41 \%$ of the fatal collisions and $28 \%$ of the injury collisions. This means that approximately 36 fatal collisions and 3,500 injury collisions occur in Ontario each year because of drivers running red-lights.

According to a comparable study in Quebec, RLR caused slightly more than 25\% of traffic injuries at signalized intersections (Brault et al., 2007). Another study in Alberta indicates that intersections on urban streets, where the speed limit is $60 \mathrm{~km} / \mathrm{hr}$ or less, are particularly dangerous; accounting for $47 \%$ of all fatalities and $57 \%$ of those seriously injured (Alberta Justice and Solicitor General, 2014).

Legislation in Ontario requires that a traffic collision, which results in death, injury, or property damage to an apparent extent of $\$ 1,000$ or more, be reported immediately to the Registrar of Motor Vehicles as required under the Highway Traffic Act (Ontario Regulation 596) (Ontario's Regulatory Registry, 2015). An officer completes a standardized collision report form which provides information on various aspects of the traffic collision. This report is based on the data collected from these report forms and summarized by City of Ottawa staff. The collision database includes information regarding the date, time, type, classification, road surface condition, vehicle 
type, vehicle maneuver, apparent driver action, road alignment and initial direction of travel date of each incident.

\subsection{RLC Enforcement Against RLR}

The purpose of the RLC is to increase safety at signalized intersections by reducing redlight violations and the resultant collisions attributable to RLR. When selecting a site for a potential RLC treatment, consideration is usually given to the potential of collision reduction (especially fatal and injury collisions), the geographic distribution, and consideration of alternative engineering countermeasures. Staff at the City of Ottawa has identified a set of site selection criteria, listed in Table 2.1, to be used in the selection of additional locations for the RLC program (Leclair, 2004). The City's first objective is to place RLCs at intersections where high numbers of angle collisions are taking place, since they result in severe type of collisions.

Once a site gets selected, the RLCs are connected to the traffic signal and to sensors that are installed in the pavement to detect vehicles. The sensors are designed to be activated only when the red-light signal is displayed for traffic proceeding in that direction. By having the loop detectors used only for the automated enforcement system, interference and conflicts with other detectors used for the traffic control system are avoided.

When the system is activated by a vehicle running a red light, two pictures are taken by the camera. The first picture shows that the front of the vehicle has just entered the intersection when the traffic signal is red. This picture must show the pavement marking defining the intersection (usually the stop bar or crosswalk), the traffic signal displaying a red-light, and the license plate of the vehicle in question (FHWA, 2009). The 
Table 2.1 - Ottawa's RLC site selection criteria and process (Adapted from Table 2 of Leclair, 2004)

\begin{tabular}{|c|l|}
\hline Step 1. & $\begin{array}{l}\text { a) Review collision data for signalized intersections. } \\
\text { b) List signalized intersections with highest frequency of serious angle type collisions. } \\
\text { c) List signalized intersections with highest frequency of serious turn type collisions. } \\
\text { d) Identify signalized intersections with highest frequency of collisions involving pedestrians } \\
\text { and cyclists. } \\
\text { e) List signalized intersections with highest frequency of rear-end type collisions. }\end{array}$ \\
\hline Step 2. & $\begin{array}{l}\text { Consult with Ottawa Police Services to determine intersections with highest issuance of Red- } \\
\text { light running infractions and highest number of requests from public for Police Enforcement. }\end{array}$ \\
\hline Step 3. & $\begin{array}{l}\text { Review short-listed locations for possible alternate solutions involving intersection } \\
\text { geometry, signal operation, signage prior to installing Red-light Cameras. }\end{array}$ \\
\hline Step 4. & $\begin{array}{l}\text { Review short listed intersections for physical suitability of Red-light Camera installation } \\
\text { (presence of above and below ground plant in addition to driveways can preclude } \\
\text { installation). }\end{array}$ \\
\hline Step 5. & $\begin{array}{l}\text { Review short listed intersections not deemed suitable for Red-light Camera enforcement for } \\
\text { possible additional Police Enforcement. }\end{array}$ \\
\hline Step 6. & Conduct 'before" Red Light running studies at short-listed intersections. \\
\hline Step 7. & $\begin{array}{l}\text { Select final locations for Red Light enforcement based on highest potential for collision } \\
\text { reduction while attempting to achieve even distribution across the City of Ottawa. }\end{array}$ \\
\hline
\end{tabular}

second picture then shows the vehicle in the intersection a short time later ( 0.5 to 1.5 seconds). To be counted as a red-light violation, a vehicle's rear tires must have been positioned behind the crosswalk or stop line prior to entering on red (Retting et al., 2007). Vehicles already in the intersection when the signal changes to red (i.e. when waiting to turn) are not counted as red-light violators. From the pictures taken, the license plate gets magnified to allow for identification. Therefore, the placement of the sensors is critical to the integrity of the RLC system and the citations developed from the photographic data.

At each camera location, only one of the typical four approaches to an intersection is usually monitored. Analysis is usually undertaken to determine the best intersection approach for RLC installation by using collision data supplemented with overall traffic volume data (Auckland Transport, 2011). If the numbers of collisions on each approach of an individual intersection are equal, then the RLC approach site is chosen based on the highest traffic volume during peak traffic times. Studies have found that collision risk 
increases on high traffic volume roads as a result of fewer gaps where subsequently drivers take more risks (Auckland Transport, 2011).

Automated RLC enforcement has the advantage of being active $24 / 7$ and therefore has a much greater deterrent effect than the occasional presence of a police officer. Manual enforcement methods are resource intensive and high risk, whereas RLCs can operate 24 hours a day and do not involve high-speed pursuits. However, one downside to automated enforcement is that a manned officer is often able to identify other serious violations at the scene of the collision, such as driving with a suspended license or drug/alcohol impairment.

RLCs are used extensively in many countries around the world including Australia, Austria, Belgium, Canada, Germany, Kuwait, New Zealand, the Netherlands, Singapore, South Africa, Switzerland, United Kingdom and the United States. In Canada, cameras are used in the Provinces of Alberta, British Columbia, Manitoba, Ontario, Quebec, and Saskatchewan (Shimizu and Desrochers, 2015).

Seven Ontario municipalities (including Ottawa) have an existing joint contract with the vendor Traffipax for the installation, operation and maintenance of every RLC in the province. The last contract expired at the end of 2016 but through a recent procurement process, Traffipax was again chosen as the successful vendor for the next contract that began on January 1, 2017. This new contract is for a 5 year-period with an optional extension to 10 years (Keech, 2016).

\subsubsection{Impact of RLC Programs from Previous Research}

The success of an RLC program in a district is measured by the reduction in the number of fatalities and serious injury related collisions. Since it is not economically feasible nor 
technically necessary to install RLCs at all signalized intersections, careful consideration must be taken when assessing the effectiveness of the installed RLCs and their effect on surrounding non-RLC intersections.

Numerous evaluations have been conducted to examine the various effects of RLCs on traffic safety. There is a large debate over whether RLCs actually make the roads safer, and whether the methods used to analyze their safety are suitable. Synectics Transportation Consultants (2003) evaluated the RLC enforcement program along with stepped-up police enforcement in Ontario, Canada. Forty-eight sites distributed throughout six municipalities were selected for the safety evaluation and benefit-cost analysis. An additional set of 12 local control intersections were selected. Collision data at the 48 sites were reviewed for a five-year period prior to the implementation of the RLCs and compared to collisions occurring during the first two years of the pilot project, using the empirical Bayes (EB) method. The study indicated that the two RLR countermeasures were effective at reducing angle collisions at the expense of rear-end collisions. Fatal and injury angle collisions decreased $25.3 \%$ while property damage only angle collisions decreased $17.9 \%$. However, there was an increase in rear-end collisions. Fatal and injury rear-end collisions increased $4.9 \%$ while property damage only rear-end collisions increased $49.9 \%$. Despite the increase in rear-end collisions, it was felt that as a whole the pilot project had been a success. It was estimated that a total of 47 fatal and injury collisions had been avoided as a result of the pilot project among the study sites.

However, one limitation is that this evaluation study cannot make any claim to the effectiveness of the RLCs on their own. This study involved an evaluation of the effectiveness of two treatments combined (RLCs and stepped-up police enforcement), as 
well as a publicity campaign. It is not possible to isolate the effect of the RLCs from the stepped-up police enforcement deployments and the publicity campaign. All the results presented in this study represented the combined effect of the treatments.

More recent statistics from several cities across Canada present results indicating that the number of collisions caused by RLR has been on the decline after installation of RLCs. Toronto has 77 active RLCs and the number of angle collisions causing fatalities, personal injuries, or property damage has reduced more than $60 \%$ (City of Toronto, 2017). The Region of Waterloo has 16 active RLCs and the numbers of angle collisions and turning movement collisions caused by disobeying traffic signals have decreased by $27 \%$ and $60 \%$, respectively (Region of Waterloo, 2010). However, the Region of Waterloo also saw a 23\% increase in rear-end collisions. Saskatoon has four active RLCs in the city and there has been a $9 \%$ reduction in injury and fatality rates at intersections with cameras, as well as a $12 \%$ decrease in right-angle collisions (City of Saskatoon, 2014). Calgary has nearly 40 intersection safety cameras (which track both RLR and a driver's speed at green lights). Between 2000 and 2013, total collisions at camera intersections decreased by 7\% and fatal collisions decreased by 100\% (Calgary Police Service, 2013).

Two meta-analysis reviews of before-after implementation of RLC studies have been conducted for intersections from around the USA (Aeron-Thomas \& Hess, 2005; Erke, 2009). Both reviews highlighted the failure of most studies to control for RTM and spillover effects, which are likely to overestimate and underestimate the collision reduction effects of RLCs respectively (Erke, 2009). Erke concluded that "RLCs may reduce collisions under some conditions, but on the whole RLCs do not seem to be a 
successful safety measure" and suggests longer yellow phases should be investigated. Aeron-Thomas \& Hess (2005) concluded that the RLCs are effective in reducing total casualty collisions at signalised intersections.

Erke (2009) found that RLCs do not seem to be a successful safety measure. This study found that the installation of RLCs had non-significant effects on the number of collisions by conducting a meta-analysis of 21 studies from mostly the USA and Australia over the period of 1996 to 2007. Results of the analysis found that overall collisions increased by $15 \%$, rear-end collisions increased by about $40 \%$ and right angle collisions reduced by about $10 \%$. It concluded that poor handling of RTM and spill-over effects was a key bias present in each previous study where collision reductions associated with the RLCs were identified.

Obeng and Burkley (2008) considered only rear-end collisions in a study and found that changes in driver behaviour when drivers see RLCs indeed offsets the advantages of RLCs by increasing rear-end collisions. Rear-end collisions result from non-uniform changes in driver behavior. Authors caution that limitations of the study design fail to account for changes in other types of collisions (notably decreases in right angle collisions) and that the generalization of these results to other cities may not be appropriate. Also, this study did not investigate whether such offsetting behaviour is time dependent and dissolves as time goes by and drivers become accustomed to the presence of RLCs.

In Queensland, Australia, a study of combined speed and RLC enforcement at intersections stated that there was an overall statistically significant collision reduction of $42 \%$ (Newstead \& Cameron, 2012). In contrast, changes in rear-end collisions and all 
other collision types were not statistically significant. Another study of combined speed and RLC enforcement by Budd et al. (2011) studied the effect of RLCs at 77 intersections in Victoria, Australia and quantified their effect on specific intersection approaches. The results showed that the estimated collision reduction was $47 \%$ when only the collisions involving vehicles travelling from the approach intersection leg where the camera was placed are considered. When collisions involving vehicles from all approaches are compared, the estimated collision reduction was $26 \%$. A distinction according to the collision type resulted in a significant decrease of $44 \%$ in angle and turning movement collisions and a $37 \%$ reduction in severe injury/fatal collisions of all types was estimated. There was no statistically significant estimated change in rear-end collisions suggesting the addition of speed enforcement to the RLR enforcement could be responsible for the lack of observed increase in rear-end collisions.

A study by Garber et al. (2007) specifically analyzed collisions caused by RLR, where most of the other studies used the broader category of all angle collisions, regardless of the cause. Using EB analysis, Garber et al. found no significant change in RLR collisions at RLC sites. A possible explanation is that the majority of RLR collisions result from unintentional, rather than intentional, RLR. For example, when intentional RLR occurs immediately after the signal changing to red, cross traffic has not been released and the likelihood of a collision is low. In contrast, unintentional RLR is hazardous because cross traffic can be in the intersection when the infraction occurs. The failure of RLCs to reduce RLR collisions is consistent with collisions occurring from unintentional RLR. Further, the Garber et al. (2007) study reveals that angle collisions 
are not a good proxy for RLR collisions since they found that RLR collisions did not significantly change at RLC sites, whereas angle collisions increased.

Estimates of the safety effect of RLCs varied considerably according to the research design and study methodology. A summary of further relevant study findings is provided in Table 2.2. The bulk of the results supports a conclusion that RLCs reduce angle collisions and could increase rear-end collisions; however, most studies are tainted by methodological difficulties that raise questions about any conclusions obtained.

Failure to account for RTM can exaggerate the positive effects, while ignoring possible spillover effects at intersections without RLC will lead to an underestimation of RLC benefits, even more so if sites with these effects are used as a comparison group. The spillover effect is the expected effect of RLCs on intersections other than the ones actually treated, resulting from jurisdiction-wide publicity and the general public's lack of knowledge of where RLCs are installed (AECOM, 2014). Almost all studies had one or the other of these flaws and many had both, in addition to other flaws such as not accounting for changes in traffic volume. 
Table 2.2 - Effectiveness of RLCs from previous studies

\begin{tabular}{|c|c|c|c|c|c|}
\hline No. & Author \& year & $\begin{array}{l}\text { Location of } \\
\text { study }\end{array}$ & Data & Methods & Main Findings \\
\hline 1 & $\begin{array}{l}\text { Retting \& } \\
\text { Kyrychenko } \\
\text { (2002) }\end{array}$ & California, USA & $\begin{array}{l}11 \text { RLC sites } \\
29 \text { months before and } 29 \\
\text { months after } \\
\text { Years: } 1995 \text { to } 1999\end{array}$ & $\begin{array}{l}\text { Generalized } \\
\text { linear regression } \\
\text { model }\end{array}$ & $\begin{array}{l}7 \% \text { reduction in total collisions } \\
32 \% \text { reduction in total right angle collisions } \\
3 \% \text { increase in total rear-end collisions } \\
29 \% \text { reduction in injury collisions } \\
68 \% \text { reduction in injury right angle collisions }\end{array}$ \\
\hline 2 & Synectics (2003) & Ontario, Canada & $\begin{array}{l}19 \text { red light camera sites } \\
17 \text { stepped-up police } \\
\text { enforcement sites } \\
12 \text { local comparison sites. } \\
\text { Years: } 2000 \text { to } 2002\end{array}$ & Empirical Bayes & $\begin{array}{l}6.8 \% \text { decrease in total injury and fatal collisions } \\
18.5 \% \text { increase in total PDO collisions } \\
25.3 \% \text { decrease in fatal and injury angle collisions } \\
4.9 \% \text { increase in injury and fatality RE collisions } \\
49.9 \% \text { increase PDO RE collisions }\end{array}$ \\
\hline 3 & $\begin{array}{l}\text { Burkey \& Obeng } \\
\qquad(2004)\end{array}$ & USA & $\begin{array}{l}303 \text { intersections } \\
57-\text { month period }\end{array}$ & $\begin{array}{l}\text { Poisson and NB } \\
\text { regression } \\
\text { models }\end{array}$ & $\begin{array}{l}40 \% \text { increase in total collisions } \\
40 \text { to } 50 \% \text { increase in PDO and injury collisions }\end{array}$ \\
\hline 4 & $\begin{array}{l}\text { Council et al. } \\
\quad(2005)\end{array}$ & USA & $\begin{array}{l}132 \text { sites ( } 7 \text { jurisdictions) } \\
\text { Years: } 3 \text { before and } 1.5 \text { after }\end{array}$ & Empirical Bayes & $\begin{array}{l}25 \% \text { reduction in angle collisions } \\
15 \% \text { increase in rear-end collisions }\end{array}$ \\
\hline 5 & $\begin{array}{l}\text { Garber et al. } \\
\quad(2007)\end{array}$ & Virginia, USA & $\begin{array}{l}28 \text { intersections with cameras } \\
\text { and } 44 \text { without } \\
\text { Several jurisdictions. } \\
\text { Years: } 1998 \text { to } 2004\end{array}$ & $\begin{array}{l}\text { The paired t-test, } \\
\text { ANOVA, GLM, } \\
\text { Empirical Bayes } \\
\text { method }\end{array}$ & $\begin{array}{l}8 \% \text { to } 42 \% \text { reduction in red light running collisions } \\
27 \% \text { to } 42 \% \text { increase in rear-end collisions }\end{array}$ \\
\hline 6 & $\begin{array}{l}\text { Sayed \& de Leur } \\
\qquad(2007),\end{array}$ & Alberta, Canada & $\begin{array}{l}25 \text { intersections } \\
\text { Before Period: } 3 \text { years After } \\
\text { Years: } 2-3 \text { years }\end{array}$ & Empirical Bayes & $\begin{array}{l}11 \% \text { reduction in total collisions } \\
17 \% \text { reduction in angle collisions } \\
12 \% \text { reduction in rear end collisions } \\
6 \% \text { reduction in severe collisions } \\
14 \% \text { reduction in PDO collisions }\end{array}$ \\
\hline 7 & $\begin{array}{l}\text { Shin \& } \\
\text { Washington } \\
\text { (2007) }\end{array}$ & Arizona, USA & 24 RLC intersections & Empirical Bayes & $\begin{array}{l}20 \%-42 \% \text { reduction in angle collisions } \\
10 \%-45 \% \text { reduction in left turn collisions } \\
41 \%-51 \% \text { increase in rear-end collisions }\end{array}$ \\
\hline
\end{tabular}




\begin{tabular}{|c|c|c|c|c|c|}
\hline 8 & Guerin (2010) & $\begin{array}{l}\text { New Mexico, } \\
\text { USA }\end{array}$ & $\begin{array}{l}20 \text { RLC intersections } \\
\text { Years: } 2004 \text { to } 2010\end{array}$ & $\begin{array}{l}\text { Before and after } \\
\text { with comparison } \\
\text { group and EB } \\
\text { method }\end{array}$ & $\begin{array}{l}3.1 \% \text { increase in total collisions } \\
5.1 \% \text { reduction in injury collisions } \\
8.3 \% \text { increase in PDO collisions }\end{array}$ \\
\hline 9 & $\begin{array}{c}\text { Auckland } \\
\text { Transport (2011) }\end{array}$ & New Zealand & $\begin{array}{l}20 \text { RLC intersections } \\
\text { Years: less than } 1 \text { year }\end{array}$ & $\begin{array}{l}\text { Before- and after } \\
\text { study }\end{array}$ & $\begin{array}{l}69 \% \text { reduction in total RLR collisions } \\
43 \% \text { reduction in RLR collisions } \\
32 \% \text { reduction in rear-end collisions } \\
93 \% \text { reduction in the social cost of collisions }\end{array}$ \\
\hline 10 & $\begin{array}{l}\text { Budd et al. } \\
\quad \text { (2011) }\end{array}$ & $\begin{array}{l}\text { Victoria, } \\
\text { Australia }\end{array}$ & $\begin{array}{l}87 \text { RLCs } 10,245 \text { collisions on } \\
1189 \text { intersections } \\
\text { Years: } 2000 \text { to } 2009\end{array}$ & $\begin{array}{l}\text { Before-after and } \\
\text { Poisson } \\
\text { regression }\end{array}$ & $\begin{array}{l}47 \% \text { reduction in total collisions RLC approach } \\
26 \% \text { reduction in total collisions all approaches } \\
37 \% \text { reduction in severe injury/fatal collisions } \\
44 \% \text { reduction in angle collisions }\end{array}$ \\
\hline 11 & De Leur (2011) & BC, Canada & $\begin{array}{l}120 \text { RLC intersections } \\
\text { Years: } 3 \text { years after }\end{array}$ & $\begin{array}{l}\text { Regression \& } \\
\text { Empirical Bayes }\end{array}$ & $\begin{array}{l}14 \% \text { reduction in injury collisions } 18 \text { months after } \\
\text { program } \\
6.4 \% \text { reduction in injury collisions } 36 \text { months after } \\
\text { program }\end{array}$ \\
\hline 12 & Hu et al. (2011) & USA & $\begin{array}{l}14 \text { RLC intersections } \\
\text { Years: } 1992 \text { to } 2008\end{array}$ & $\begin{array}{l}\text { Before and after } \\
\text { with comparison } \\
\text { group }\end{array}$ & $24 \%$ reduction in fatality collisions \\
\hline 15 & $\begin{array}{c}\text { Newstead \& } \\
\text { Cameron (2012) }\end{array}$ & $\begin{array}{l}\text { Queensland, } \\
\text { Australia }\end{array}$ & $\begin{array}{l}142 \text { RLC intersections } \\
\text { Years: } 2008\end{array}$ & $\begin{array}{l}\text { Poisson, NB \& } \\
\text { Empirical Bayes } \\
\text { models }\end{array}$ & $\begin{array}{l}23 \% \text { reduction in all collisions } \\
24 \% \text { reduction in fatal and hospitalisation }\end{array}$ \\
\hline 16 & Нøуе (2013) & USA & 28 studies & $\begin{array}{l}\text { Various (meta- } \\
\text { analysis) }\end{array}$ & $\begin{array}{l}33 \% \text { reduction in angle collisions } \\
19 \% \text { increase in rear-end injury collisions } \\
39 \% \text { increase in rear-end collisions }\end{array}$ \\
\hline 17 & Ko et al. (2013) & Texas, USA & 245 intersections & Empirical Bayes & $\begin{array}{l}20 \% \text { reduction in total collisions } \\
24 \% \text { reduction in angle collisions } \\
37 \% \text { increase in rear-end collisions }\end{array}$ \\
\hline
\end{tabular}




\begin{tabular}{|c|c|c|c|c|c|}
\hline 18 & AECOM (2014) & Alberta, Canada & $\begin{array}{l}76 \text { RLC intersections } \\
141 \text { non-RLC signalized } \\
\text { intersections } 37 \text { un-signalized } \\
\text { sites }\end{array}$ & Empirical Bayes & $\begin{array}{l}8.4 \% \text { reduction in total collisions } \\
37.7 \% \text { reduction in right-angle collision } \\
32.4 \% \text { reduction in severe collisions } \\
7.7 \% \text { increase in rear-end collisions } \\
1.4 \% \text { increase in PDO collisions }\end{array}$ \\
\hline 19 & Boudreaux (2014) & Florida, USA & Years: 2010 to 2011 & $\begin{array}{l}\text { Poisson and NB } \\
\text { regression }\end{array}$ & $\begin{array}{l}1.3 \% \text { reduction in total collisions } \\
46 \% \text { decrease in fatal collision }\end{array}$ \\
\hline 20 & $\begin{array}{l}\text { De Pauw et al. } \\
\quad \text { (2014) }\end{array}$ & Belgium & $\begin{array}{l}\text { Combined speed and red light } \\
\text { cameras at } 253 \text { intersections } \\
\text { Years: } 2002 \text { to } 2007\end{array}$ & $\begin{array}{l}\text { Before- and after } \\
\text { study with } \\
\text { control for the } \\
\text { trend }\end{array}$ & $\begin{array}{l}5 \% \text { increase in injury collisions } \\
14 \% \text { reduction in severe collisions } \\
6 \% \text { reduction in side collisions } \\
44 \% \text { increase in rear-end collisions } \\
12 \% \text { reduction in right-angle collision }\end{array}$ \\
\hline 21 & $\begin{array}{c}\text { Lord \& } \\
\text { Geedipally (2014) }\end{array}$ & Chicago, USA & $\begin{array}{l}90 \text { RLC intersections } \\
56 \text { non-RLC intersections } \\
\text { Years: } 3 \text { yrs before } 3 \text { yrs after }\end{array}$ & Empirical Bayes & $\begin{array}{l}5 \% \text { increase in total injury collisions } \\
15 \% \text { reduction in angle and turning injury collisions } \\
22 \% \text { increase in rear-end injury collisions }\end{array}$ \\
\hline 22 & $\begin{array}{l}\text { Currie \& Basak } \\
\qquad(2015)\end{array}$ & Toronto, Ontario & $\begin{array}{l}77 \text { RLC intersections } \\
\text { Years: } 2007 \text { to } 2014\end{array}$ & $\begin{array}{l}\text { Before- and after } \\
\text { study }\end{array}$ & $\begin{array}{l}40 \% \text { reduction in angle (fatal) collisions } \\
27 \% \text { increase in rear-end (PDO) collisions } \\
14 \% \text { reduction in rear-end (injury) collisions } \\
19 \% \text { reduction in PDO collisions } \\
37 \% \text { reduction in injury collisions }\end{array}$ \\
\hline 23 & Keech (2016) & Kingston, Ontario & $\begin{array}{l}\text { 10 RLC intersections } \\
\text { Years: } 2016\end{array}$ & $\begin{array}{l}\text { Before- and after } \\
\text { study }\end{array}$ & $\begin{array}{l}25 \% \text { reduction in angle collisions } \\
15 \% \text { increase in rear-end collisions }\end{array}$ \\
\hline 24 & Cohan (2017) & Delaware, USA & $\begin{array}{l}30 \text { RLCs intersections } \\
\text { Years: } 3 \text { yrs before } 10 \text { yrs } \\
\text { after }\end{array}$ & $\begin{array}{l}\text { Before- and after } \\
\text { study }\end{array}$ & $\begin{array}{l}6 \% \text { increase in total collisions } \\
47 \% \text { reduction in angle collisions } \\
9 \% \text { increase in rear-end collisions } \\
24 \% \text { decrease in red-light running collisions }\end{array}$ \\
\hline 25 & $\begin{array}{l}\text { Mahmassani et al. } \\
\text { (2017) }\end{array}$ & Chicago, USA & $\begin{array}{l}151 \text { RLC intersections } \\
\text { Years: } 2008 \text { to } 2009\end{array}$ & Empirical Bayes & $\begin{array}{l}10 \% \text { reduction in total collisions } \\
19 \% \text { reduction in angle collisions } \\
14 \% \text { increase in rear-end collisions }\end{array}$ \\
\hline
\end{tabular}




\subsubsection{Spillover Effect and Collision Migration}

It is important to find out whether RLCs affect the safety performance only at the specific intersections at which RLCs are installed, or if their presence influences the behaviour of drivers in general. RLCs can improve safety at other signalized intersections, not equipped with RLCs, by creating a general deterrence of signal violation leading to collision reductions (Christie et al., 2003). Figure 2.3 illustrates this type of behavioural influence, where drivers carry over their careful behaviour from RLC intersections to non-RLC intersections, referred to as spillover or halo effect.

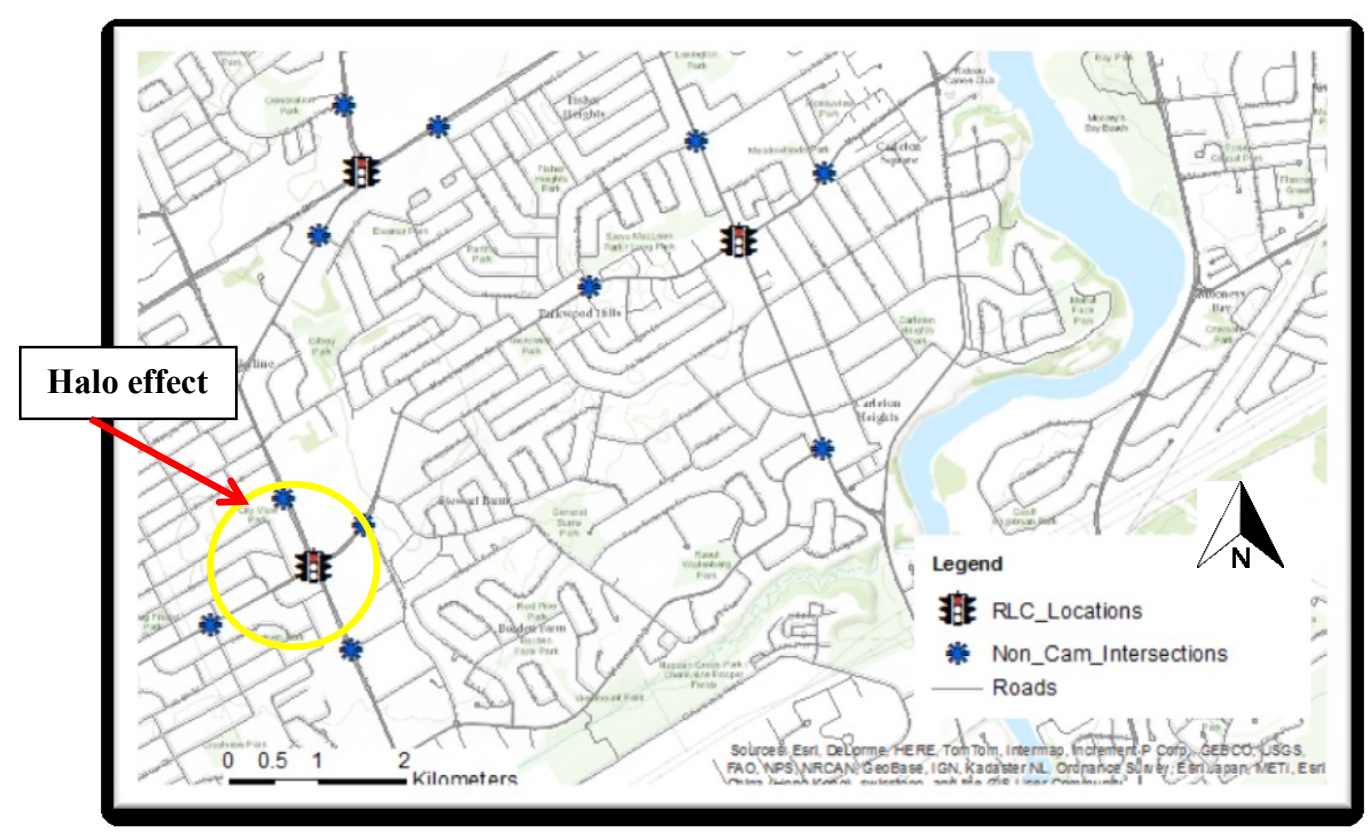

Figure 2.3 - Spillover effect

On the other hand, RLCs implemented at a particular location may be followed by a migration of collisions to adjacent intersections. Crash migration is the phenomenon that the collision frequency or collision severity apparently rises at the untreated sites adjacent to treated sites as a result of the treatment (Shen \& Gan, 2003). There are two mechanisms speculated by which collisions might increase at other locations due to sitespecific RLC enforcement: (1) If the enforcement zone is widely known, motorists may 
decrease speeds near the intersection sites, perhaps abruptly, and increase their speeds before or after the treated intersections to make up for lost time. (2) Drivers may choose alternate routes to avoid treated intersections, and therefore, collision frequencies at treated sites may decline and move to the surrounding intersections. Safety assessments should be performed to individual intersections within a roadwork to help avoid missing potential system-wide effects.

Therefore, the examination of collisions at surrounding intersections can help determine the true effect of RLCs. The spillover effect does not happen immediately, as the connection to the public consciousness through violation notices, signage at RLC sites, word-of-mouth and through formal and informal media takes a significant period of time (Solomon, 2011). Wilson et al. (2006) defines a time-halo as the length of time an effect of enforcement is present after detection devices have been installed. The distancehalo is the distance from the point of enforcement that the decrease in RLR continues. Time and distance-halos should be computed to investigate the local and widespread effects of an enforcement.

Studies reporting halo effects varied in terms of location, duration of intervention, data collection and measurement. The distance halo of stationary policing appears to be in the range of 1.5 miles $(2.4 \mathrm{~km})$ to 5 miles $(8 \mathrm{~km})$ from the enforcement site (Elliott and Broughton, 2005).

Distance-halos of speed cameras have been reviewed in the literature, yet no research has been conducted related to RLCs. Studies indicate that $500 \mathrm{~m}$ is about the maximum distance halo of a speed camera (Elliott and Broughton, 2005; Champness and Folkman, 2005). Mountain and Fawaz (1992) used $500 \mathrm{~m}$ as a limit for selecting the sites 
surrounding automated speed cameras and concluded that crash migration did occur because of treatment and that the area of influence varied with time and distance from the treated site. However, there was no explanation in the report as to why the distance of $500 \mathrm{~m}$ was chosen. Keenan (2002) also looked at $500 \mathrm{~m}$ before automated speed cameras and $500 \mathrm{~m}$ after. The study found that by $500 \mathrm{~m}$ after passing a speed camera mean speeds increased to the same levels they were $500 \mathrm{~m}$ before the camera. At these points, around $80 \%$ of drivers were exceeding the speed limit. This finding is consistent with previous research by Nilsson (1992) who also found that the distance halo effect due to speed cameras was $500 \mathrm{~m}$. However, no published studies evaluating distance halos for RLCs were found.

A study by Malone et al. (2010) found that the possible spillover effects of the RLC program in Alberta, Canada demonstrated a moderate, yet significant, effect on angle collisions based on trend analysis. Collisions at non-RLC equipped signalized intersections were found to significantly decrease by $8.6 \%$ in the after-period, representing a reduction of 25 right angle incidents. Another study in Alberta, Canada (AECOM, 2014) separated the spillover effect of RLCs and determined that the effect was significant across the entire series of jurisdictions studied. That is, there was a significant safety impact on intersections without RLCs. However, no specific spillover distances were mentioned in the report.

Studies on the effectiveness of speed camera enforcement in Fairfax, Virginia, Oxnard California, Scottsdale, Arizona, and Cambridgeshire, UK also found evidence of spillover effects (Retting \& Kyrychenko 2002; Shin \& Washington 2007; Hess 2004; Erke, 2009). Although there is some evidence of a spillover effect, some studies indicate 
no such effect was found (Hillier et al., 1993, Garber et al., 2007). Specific time and distance-halos resulting from the RLC treatment have not been discussed in the literature and need further investigation.

\subsection{Statistical Modeling of Safety Performance}

Accurate estimation of countermeasure effectiveness is critical in transportation safety. Analytic methods are usually utilized to attempt to quantify the effect of an intervention on an outcome. This thesis focuses on observational methods, since there was no involvement from the researcher on the use or change of the RLC intervention. Three commonly used observational methods to determine the effectiveness of traffic safety countermeasure are cross-sectional regression studies (e.g. negative binomial (NB)), observational before-after studies (i.e. EB), and case-control studies (i.e. propensity score matching) (Sasidharan \& Donnell, 2013).

Traditional cross-sectional studies are known as prevalence studies and do not have an inherent temporal dimension. These studies evaluate subjects at one point in time. Observational before-after are usually retrospective studies where they look back in time to examine causal associations. Case-control study designs are also retrospective and assess the history of the subject for the presence or absence of an exposure. It is important to recognize the strengths and limitations associated with the various methods to help identify a suitable method given the constraints of each specific evaluation. Table 2.3 summarizes the strengths and limitations of each method used to analyze observational data in traffic safety. The application of these methods will be discussed further in the next chapter. 
Table 2.3 - Methods used to analyze observational data in traffic safety

\begin{tabular}{|c|c|c|c|}
\hline $\begin{array}{l}\text { Analytic } \\
\text { approach }\end{array}$ & About the method & Strengths & Limitations \\
\hline $\begin{array}{c}\text { Cross- } \\
\text { sectional }\end{array}$ & $\begin{array}{l}\text { Is a type of regression in which } \\
\text { the explained and explanatory } \\
\text { variables are associated with } \\
\text { one point in time. }\end{array}$ & $\begin{array}{l}\text { 1. Produces specific } \\
\text { coefficients for each } \\
\text { individual confounder which } \\
\text { allows examination of these } \\
\text { specific effects on the } \\
\text { outcome. } \\
\text { 2. Useful when the before } \\
\text { period data are not available; } \\
\text { data exhibits over-dispersion; } \\
\text { or, the countermeasure } \\
\text { installation date is unknown. }\end{array}$ & $\begin{array}{l}\text { 1. Not useful for rare events } \\
\text { because in a one-time snapshot } \\
\text { (rare outcomes or rare exposures } \\
\text { may not be captured) } \\
\text { 2. Does not permit distinction } \\
\text { between cause and effect. }\end{array}$ \\
\hline EB & $\begin{array}{l}\text { Uses a control group to estimate } \\
\text { a safety performance function } \\
\text { (SPF). A SPF is used in } \\
\text { combination with observed } \\
\text { "before" collisions at the treated } \\
\text { sites to predict the number of } \\
\text { collisions that would have been } \\
\text { expected in the after period for } \\
\text { the treated entities had the } \\
\text { treatment not been } \\
\text { implemented. This prediction is } \\
\text { then compared to the expected } \\
\text { number of collisions in the after } \\
\text { period with the treatment. }\end{array}$ & $\begin{array}{l}\text { 2. Accounts for differences in } \\
\text { traffic volume and collision } \\
\text { trends between the before and } \\
\text { after periods at the treatment } \\
\text { sites. }\end{array}$ & $\begin{array}{l}\text { 1. Large sample sizes and long } \\
\text { follow-up periods (years of data) } \\
\text { are necessary. } \\
\text { 2. No guidelines exist concerning } \\
\text { how to most effectively select the } \\
\text { group of reference sites for } \\
\text { developing SPFs. } \\
\text { 3. The statistical significance of } \\
\text { observed changes cannot be } \\
\text { evaluated. }\end{array}$ \\
\hline $\begin{array}{c}\text { Case- } \\
\text { control }\end{array}$ & $\begin{array}{l}\text { Match an untreated group to the } \\
\text { treated group such that the } \\
\text { matched group is comparable to } \\
\text { the treated group in all aspects } \\
\text { of characteristics except the } \\
\text { treatment. The observed } \\
\text { outcome for the matched group } \\
\text { can then be used as the } \\
\text { counterfactual, and the } \\
\text { treatment effect is estimated } \\
\text { simply as the difference } \\
\text { between the average outcomes } \\
\text { of the two groups. }\end{array}$ & $\begin{array}{l}\text { 1. Feasible method for } \\
\text { studying rare outcomes or } \\
\text { those with long lag between } \\
\text { exposure and outcome. }\end{array}$ & $\begin{array}{l}\text { 1. Only adjusts for measured } \\
\text { confounders. }\end{array}$ \\
\hline
\end{tabular}

With traditional cross-sectional data not much can be inferred about intraindividual change over time. When all variables are measured at the same time point, it is frequently not possible to establish whether causal influence occurs. In addition to the various statistical models and estimation tools available for count data, such as Poisson estimation and NB regressions, further advancements have been made to improve the statistical models by using panel count data (Yaacoub et al., 2011). 
When dealing with panel type data of traffic collision counts, for example, both collision frequency and severity often have observations that are in close spatial or temporal proximity (data from the same geographic region or data collected from the same observational unit over successive time periods). All data are likely to have unobserved factors that may influence the frequency and/or severity of collisions and, because these unobserved factors are likely to be correlated over space and time (Mannering and Bhat, 2014). Ignoring the spatial and temporal correlation of data will most likely result in inefficient and possibly inconsistent parameter estimates. Examples of such unobserved factors could be pavement irregularities that may not be observed but may extend over time or space, climate effects that may result in reduced pavement friction over time and space, local sight-distance restrictions that again may extend over time and space.

To account for these spatial and temporal correlations, random-effects (RE) and fixed-effects (FE) regression models can be considered. These models were first examined by Hausman et al. (1984). Random-effect models are used where the common unobserved effects are assumed to be distributed over the spatial/temporal units according to some distribution and the shared unobserved effects are assumed to be uncorrelated with explanatory variables. With count data, a suitable model distribution may be a random-effects negative binomial (RENB) regression.

Fixed-effect models are used where common unobserved effects are accounted for by indicator variables and the shared unobserved effects are assumed to be correlated with independent variables. A FE model examines if intercepts vary across group or time 
period, whereas a RE model explores differences in error variance components across individual or time period (Park, 2011).

These modeling approaches have several advantages over cross-sectional models, particularly, for their ability to account for heterogeneity in the data (Yaacoub et al., 2011). It is important to determine whether there are genuine differences underlying the results of the studies (heterogeneity), or whether the variation in findings is due to random error or chance alone (homogeneity) (Higgins et al., 2003).

Previous work on panel count models in traffic collision analysis applied both FE and RE models, as suggested by Hausman et al. (1984). Fixed-effect models have been studied by a number of researchers including Eisenberg (2004) who examined the relationship between precipitation and traffic collisions in 48 contiguous states in the US using a FENB model for the period of 1975 to 2000. Karlaftis and Tarko (1998) employed FE Poisson and FENB to develop a collision model based on 6-year collision data set for urban and suburban arterials of 92 counties in Indiana. Noland (2003) also used the FENB models to study the impacts of roadway infrastructure improvements on fatal and injury collision frequencies based on the aggregate state-wide and county-level data.

Shankar et al. (1998) first developed a random-effects negative binomial (RENB) model to investigate the impacts of geometric and traffic factors on median crossover collision frequency in Washington State. Random-effects models have also been studied by a number of other researchers including Johansson (1996) who studied the effect of a lowered speed limit on the number of crashes on roadways in Sweden. Miaou et al. (2003) used RE in the development of collision-risk maps in Texas. However, no 
research has been conducted utilizing the FE and RE models to assess the treatment effects of RLCs on the different traffic collision impact types.

Many studies that aim to estimate the effect of a treatment using the basic NB method and the EB method suffer from selection bias, where the units who receive the treatment may have different characteristics from those in the control group. These preexisting differences between the groups must be controlled to obtain approximately unbiased estimates of the effects of interest. It is crucial to try to separate out the causal effect of the treatment from the effect of these pre-existing differences between the treated and control groups. However, in previous research, not only is there insufficient justification of the selection of control groups, how the treatment and control groups are matched is also unclear.

Given the limitations associated with the EB and cross-sectional methods, alternative evaluation methods are needed to estimate the safety effectiveness of the RLCs. The propensity score matching (PSM) method has become a popular approach to estimate causal treatment effects by modeling the relationship between the covariates and the treatment assignment. The PSM approach is used to tackle the critical issue of choosing an appropriate control group to evaluate the effect of RLCs on the reduction of road collisions. What makes the PSM method attractive is that it gives a clear criterion, the propensity score, by which to select the control group. The PSM method has been widely used in the multiple fields, such as medicine (Stone et al. 1995; Gum et al. 2001; and Akematsu et al. 2012), communications (Kincaid 2009), economics (Lechner 2002), and policy evaluation (Harknett 2006). 
PSM is a relatively new method that has had few applications in traffic safety. One of first applications of propensity scores in traffic safety research (Davis, 2000) argued that the selection mechanism for the treatment should be included as a part of a traffic safety evaluation. It suggested that, when this does not occur, both EB and crosssectional analysis methods become inconsistent with the true outcome. Other applications of propensity scores in traffic safety include estimating child safety restraint effectiveness (Elliott et al., 2009), estimation of design exception safety impacts (Wood \& Porter, 2013), safety effects of intersection lighting (Sasidharan \& Donnell, 2013) and assessing the impacts of speed cameras on safety (Li et al., 2013). Though widely implemented in diverse disciplines and in many contexts, to the best of the authors' knowledge, it remains untried in RLC safety research by traffic management scholars. It should be considered as an additional tool available to investigators when estimating treatment effects in studies where potential bias may exist.

The overall objective of the PSM method is to replicate a randomized experiment in two ways: (1) by comparing treated and control units that look as if they could have been randomly assigned to treatment or control groups; and (2) by forming the comparison groups without the use of the outcome, thus preventing intentional or unintentional bias in selecting a particular sample to achieve a desired result.

The primary objective of a treatment evaluation is to confirm and quantify the treatment effect. Although important, the final result is usually a conclusion with respect to the average treatment effect on the entire study population. However, treated units may not be different from the untreated units when examining the overall study population, but treatment may still be better for a subset of the population. Identifying the treatment 
and covariate interactions may provide valuable information for determining this subgroup of the population. In the traffic studies reviewed in the literature review, interaction terms were not explored related to the RLC effectiveness. Therefore, adding interaction terms to the statistical models can greatly expand understanding of the relationships among the treatment and road factors. Interaction terms are used to test how a dependent variable changes when an independent variable changes conditional on the presence or magnitude of another independent variable (Drichoutis, 2011).

\subsection{Economic Effect of RLC}

The annual operating financial cost of the RLCs in Ottawa is estimated at around $\$ 30,000$ to $\$ 35,000$ per camera (Foote, 2016). This cost includes monthly payments to the vendor (Traffipax) for the installation, operation and maintenance of the cameras, and payments to the City of Toronto processing centre (once per year) and the Ministry of Transportation Ontario (four times per year) for vehicle ownership information.

No current benefit-cost reports were published assessing the effectiveness of RLCs in Ottawa. The only public report found analyzing Ottawa's RLC program was from 2003 (Synectics, 2003), which estimated a benefit-cost ratio of 1.57, where the net benefits in collision reductions exceeded the operating costs. This study, however, cannot make any claim to the effectiveness of the RLCs on their own as this evaluation study involved an evaluation of the effectiveness of RLCs with stepped-up police enforcement as well as this was a combined result from other cities from around Ontario.

A review was conducted of the economic effects of RLCs from other cities in Canada with a similar RLC programs to Ottawa. The RLC program in the city of Toronto also started in 2000 and currently comprises 77 RLC intersections. Since 2007, angle 
collisions resulting in fatality/injury and property-damage have decreased between $19 \%$ and $40 \%$. Net revenues generated through the program are approximately $\$ 11,500,000$ and associated societal cost savings have been estimated at over $\$ 58$ million (City of Toronto, 2015).

Several other studies have shown a reduction in both the injury severity and cost of collisions when comparing the results of the before and after installation periods for RLCs. A study of 132 RLC intersections from across seven jurisdictions in the USA showed a total reduction in right angle collisions of $24.6 \%$, a reduction in right angle injury collisions of $15.7 \%$, a total increase in rear-end collisions of $14.9 \%$, an increase in rear-end injury collisions of $24.0 \%$, and a total crash cost savings of $\$ 38,000$ per RLC intersection per year (Council et al., 2005). The jurisdictions chosen for this study were El Cajon, San Diego, and San Francisco, CA; Howard County, Montgomery County, and Baltimore, MD; and Charlotte, NC.

A study in Phoenix, AZ by Washington and Shin (2005) found the installation of RLCs effectively reduced the total amount of angle collisions by $14 \%$ and increased the rear-end collisions by $20 \%$ at 10 RLC intersections. The total number of collisions was essentially unchanged as a result of the RLCs. The expected safety net benefit was calculated at $\$ 4,504$ per year (for the 10 target approaches). There was a relatively large variability in the benefit from RLCs across Phoenix intersections, with several intersections that revealed a negative expected benefit.

The Washington and Shin (2005) report also studied the effectiveness of RLCs in Scottsdale, AZ which showed a reduction in angle collisions by $20 \%$ and increased the rear-end collisions by $41 \%$ with RLCs for 14 sites. The total number of collisions were 
slightly reduced by about $11 \%$. The expected net safety benefit at the 14 target approaches was estimated at $\$ 684,134$ per year where the RLCs in Scottsdale contributed more to decreasing fatal and injury angle and left-turn crashes than to decreasing the PDO crashes of those crash types.

The implementation of RLCs in Missouri, USA reduced overall angle collisions by $11.6 \%$, whereas rear-end crashes increased by $16.5 \%$. The net economic collision cost benefit of the implementation of RLCs was $\$ 35,269$ per site per year in 2001 dollars (approximately $\$ 47,000$ in 2016 dollars). Thus, RLCs produced a sizable net positive safety benefit that is consistent with previous statistical studies (Boris \& Carlos, 2016).

An evaluation of RLC enforcement in Fairfax County, Virginia conducted by Ruby (2001) concluded that the reduction of collisions saved $\$ 12.8$ million over the eight-year life cycle of the system.

A report by Chen et al. (2012) presents results of an evaluation of RLCs conducted between July 7 and August 8, 2010 in the Perth, Western Australia. The results found that the RLCs significantly reduced all reported collisions; angle, turning movement, rear-end and serious injury collisions by $19 \%, 41 \%, 20 \%$ and $72 \%$, respectively. After accounting for the initial project costs of $\$ 1.65$ million $(\$ 150,000$ per site) and operating and maintenance costs, the net cost savings to the community were estimated as $\$ 2.29$ million. This is the equivalent of a benefit-cost ratio of 1.8 .

In addition to collision reductions, other possible benefits or costs of implementing safety programs are changes in delays at intersections, resulting in effective increases or reductions in road capacity. These changes affect travel times for 
roadway users and should be counted properly in benefit-cost ratios. Other benefits include reduced fuel use as well as reduced pollution from vehicle emissions.

\subsection{Limitations Identified in the Literature}

The methodologies used to assess the effectiveness of RLC programs have varied, as have the conclusions drawn from the different reviewed studies. Many safety-related factors such as traffic volumes and approach speeds are uncontrolled during the periods of observation. It is important to identify the geometric and operational characteristics of intersections that could adversely affect the safety impacts of RLCs. The existence of a causal link between the RLC enforcement and road collisions remains unclear.

The lack of formal causal models makes it difficult fully to address issues such as confounding effects and RTM bias. RTM is a statistical phenomenon that may occur when the extreme cases chosen in one period are very likely to experience lower collision frequencies in the next period, and vice versa, because of random variation (FHWA, 2010). Simply stated; the highest get lower and the lowest get higher. Two key factors that together may allow this to occur are that the initial data set must be small, and that the data within this sample must be unrepresentative of the population by chance (Auckland Transport, 2011).

While the EB method has been used to account for the RTM effect, the validity of the EB approach relies heavily on the availability of an appropriate reference group. There is insufficient justification of the selection criterion and how the treatment and control groups are matched in the literature, thereby making the estimator inconsistent (Davis, 2000). Also, the EB approach requires a large sample size of data pertaining to all 
types of intersections for developing SPFs required for the EB-adjusted collision frequency estimation.

Traditional cross-sectional data and methods applied in the literature ignore the spatial and temporal correlation of data which can result in inefficient and possibly inconsistent parameter estimates when assessing the RLC effectiveness. This thesis is an attempt to overcome these limitations by setting up spatial panel data and applying statistical methods to accommodate the nature of the data.

Another limitation in the literature is that most studies that have assessed the effectiveness of RLCs have conducted their analysis for an after period which is usually fairly close to installation of the cameras. It is not well understood if the cameras have the same impact over time. In some cases, countermeasures become less effective over a long period time because drivers become accustomed to the treatment. On the other hand, enforcement may be more effective over time. Drivers who speed or run red-lights may change their behavior when they or someone they know receives a ticket. Therefore, the evaluation of traffic safety interventions often requires the collection of time series data so that a meaningful change in the series will likely be detected.

Furthermore, the existing literature is vague about the existence of spillover effects, as it fails to provide a range of magnitudes regarding distance and time-halos to help determine the local and widespread effects of the RLC enforcement. More research is needed to shed light on spillover effects of RLC programs.

Another limitation observed in most studies was that evaluations were performed city wide, hence it is not possible to evaluate or comment on the effectiveness of 
treatments at specific intersections. There is a need to more clearly evaluate the program on an individual RLC site basis.

As part of the economic analysis assessments, due to the wide variety of the collision costing methods, data accuracy, availability and the interests of the agency examining collision costs, there is a wide range in the values that have been generated for collision costs. Values can vary from a low of approximately $\$ 1$ million to a high of nearly \$20 million for a fatal collision (De Leur, 2010). Estimated collision social costs have not been challenged in the economics literature. Conducting sensitivity analysis will present the effect of varying the assumptions relating to the calculated collision reduction frequencies and the estimated social costs from previous studies.

Many researchers and journals place a strong emphasis on the sign and statistical significance of effects, but often there is very little emphasis on the substantive and practical significance of the findings. It should he recognized that no two intersections will be the same in terms of all road environment factors. Therefore, the introduction of interaction terms with the RLC presence is valuable and not found in the literature. It is important to identify the subgroups of intersections which the RLC treatment is likely to have the largest effect on and would benefit the most. It is also very important to identify those intersections for which treatment may be harmful and refrain from treating such intersections. 


\section{Methodology}

One of the most important elements of any evaluation process is the methodology that will be followed. It consists of the study design and the type of statistical analysis employed. The evaluation methodology is selected in this section is based on the available data and the resources available to conduct the evaluation. Section 3.1 discusses the collision prediction methodologies, Section 3.2 reviews the spillover effect methodology and Section 3.3 discusses the economic analysis methodology.

\subsection{Collision Prediction Methods}

A collision prediction model (CPM) is a mathematical formula describing the relationship between the safety level of existing roads or intersections and various traffic and geometric variables that explain this level (Sayed \& de Leur, 2008). The aim is to build a reliable model that fits the data well, simple enough to be interpretable and useful in practice. As with all statistical methods, the reliability of the model is partially a function of how well the model fits the original data and partially a function of how well the model has been calibrated to local data (HSM, 2010). Figure 3.1 presents a flow chart of the traffic safety modeling techniques that will be examined in this thesis to estimate the RLC effectiveness, where the results obtained will be compared.

\subsubsection{Cross-Sectional Methods}

Generalized linear models (GLMs), such as Poisson and NB regression models, have become a popular approach in investigating the relationship between collision frequency and various covariates in cross-sectional studies. The GLMs accommodates for nonnegative discrete data. The Poisson model assumes that the conditional variance 


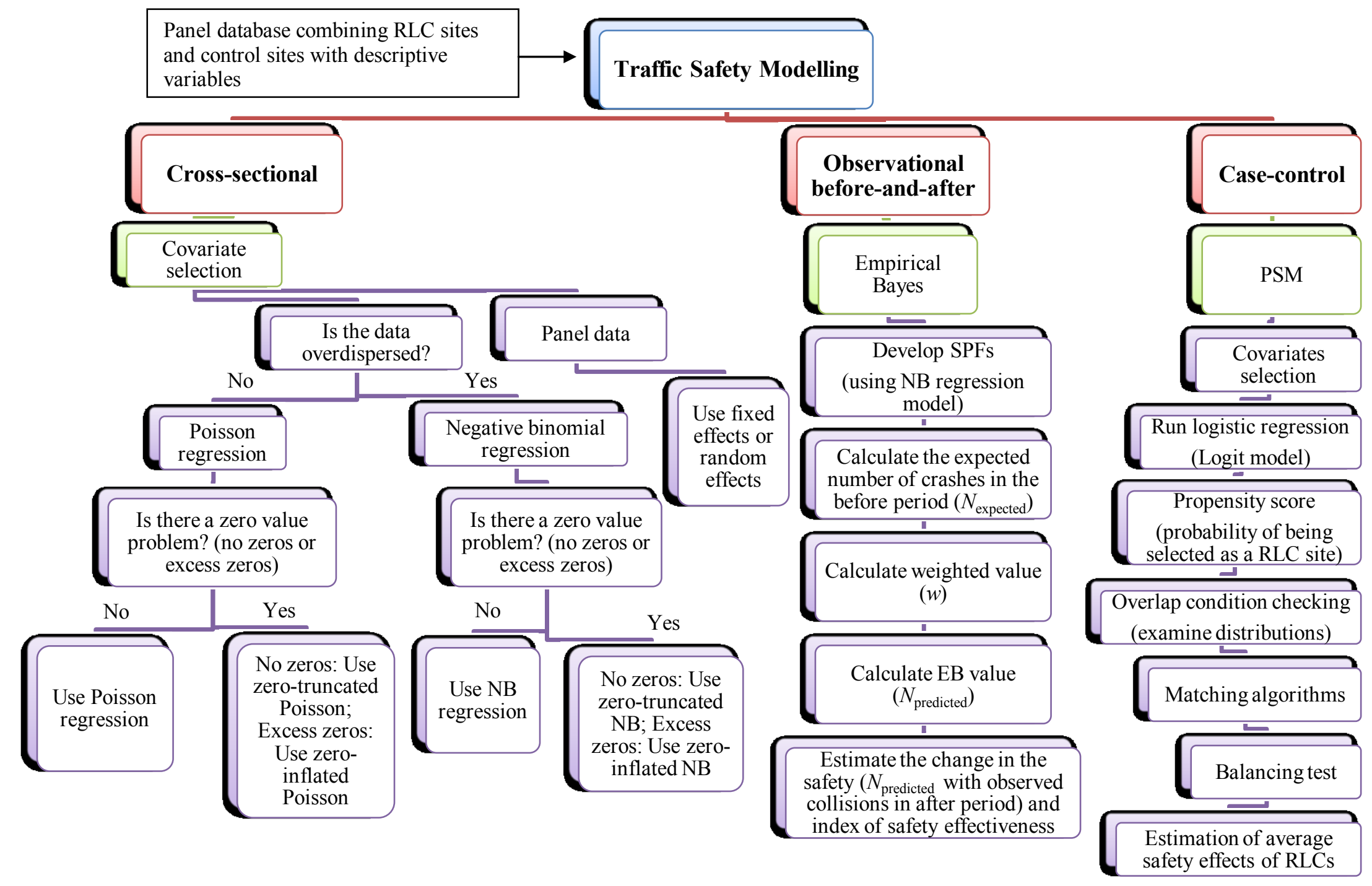

Figure 3.1 - Traffic safety statistical modeling techniques 
of the dependent variable is equal to the conditional mean. In most count data sets, however, the conditional variance is greater than the conditional mean, a phenomenon known as over-dispersion (Lord and Mannering, 2010). Therefore, the Poisson regression model often fits count data poorly, as indicated by a deviance or Pearson chi ${ }^{2}$ test. The NB regression model can be used to overcome problems associated with over-dispersed data by adding an extra term to the variance that accommodates over-dispersion. The negative binomial regression model is assumed to take the general form presented in eq. 3.1 adapted from (Donnell et al., 2009):

$$
\lambda=\exp \left(\omega+\sum X_{i} \beta_{i}+\sum L_{i} \theta_{i}+\varepsilon_{i}\right)
$$

where $\lambda=$ expected number of collisions on a roadway segment/intersection; $\omega=$ intercept; $X_{i}=$ variables influencing $\lambda$ (e.g., traffic, road geometry, etc.); $\beta_{i}=$ coefficients quantifying the relationship between $X$ and $\lambda ; L_{i}=$ a binary (dummy) variable indicating the presence of the treatment; $\theta_{i}=$ coefficient quantifying the relationship between $L$ and $\lambda ; \varepsilon_{i}=$ is a random error that is assumed to be uncorrelated with $x$ and $L$. The dependent variable, $\lambda$, considered in the model is the frequency of traffic collisions. The regression coefficients (i.e. $\beta_{i}$ and $\theta_{i}$ ) are estimated using the method of maximum likelihood.

When the dependent variable is a count variable of a rare event and is overdispersed, the use of the NB distribution is a good way of answering the question of how often does the event occur. By itself, however, it does not answer the question of what predicts whether the event occurs at all or not. Using the ZINB regression will address this issue. 
The idea is that there are two ways to think of the dependent variables: (1) that one set of variables predicts whether an event occurs at all or does not, and (2) another set of variables predicts how often the behavior occurs. In other words, what predicts what intersections will have more collisions rather than less may be different from what predicts what intersections will have collisions at all or not at all. Therefore, it is imperative to test whether using the zero-inflated model is necessary. This can be achieved by conducting the Vuong test (Vuong 1989).

Zero-inflated models operate on the principle that the excess zero density that cannot be accommodated by a traditional count structure is accounted for by a splitting regime that models a collision-free versus a collision prone propensity of a intersection (Lord and Mannering, 2010). Such models assume that the data are a mixture of two separate data generation processes: one is governed by a binary distribution that generates only zeros, and the other is either a Poisson or a NB data-generating process that generates counts, some of which may be zero (Allison, 2012 \& Lord and Mannering, 2010). The two model components of the zero-inflated Poisson (ZIP) model are described as follows (Lord et al., 2005):

$$
\begin{aligned}
& P\left(y_{j}=0\right)=\alpha+(1-\alpha) e^{-\lambda} ; n=0 \\
& P\left(y_{j}=n_{i}\right)=(1-\alpha) \frac{e^{-\lambda} \lambda^{\mathrm{n}}}{n !} ; n \geq 0
\end{aligned}
$$

where the outcome variable $y_{j}$ has any non-negative integer value, $\lambda_{i}$ is the expected Poisson count for the $i$ th individual; $\alpha$ is the probability of extra zeros. The first part of the equation has a mean of zero and the second part of the equation has a mean that depends on the covariates. The Vuong statistic (Vuong, 1989) is used to measure whether the ZIP or ZINB is appropriate for fitting the datasets. A likelihood ratio test is conducted 
to compare the NB model and ZINB model fits (by taking twice the positive difference in the log-likelihoods) (Allison, 2012).

Traditional cross-sectional collision frequency models with large temporal scales and aggregated variables are not sufficient to capture the time-varying nature of some of the road factors, causing significant loss of critical information on collision frequency modeling (Chen et al., 2016). Therefore, FE and RE models will be also examined as an extension of the NB method. The functional forms of FE and RE models are (TorresReyna, 2007):

Fixed-effect model: $\quad \lambda_{i t}=\exp \left(\omega+\sum X_{i t} \beta_{i t}+\sum L_{i t} \theta_{i t}+u_{i t}\right)$

Random-effect model: $\quad \lambda_{\mathrm{it}}=\exp \left(\omega+\sum \mathrm{X}_{\mathrm{it}} \beta_{\mathrm{it}}+\sum \mathrm{L}_{\mathrm{it}} \theta_{\mathrm{it}}+\mathrm{u}_{\mathrm{it}}+\varepsilon_{\mathrm{it}}\right)$ where $i=$ entity, $t=$ time, $u_{i t}=$ the between-entity error term specific to individual (group) or time period that is not included in the regression and the $\varepsilon_{i t}=$ the within-entity error term. Fixed-effects regression model is used to control for omitted variables that differ between cases but are constant over time. They accomplish this by introducing an additional parameter $\left(u_{i t}\right)$ for each individual in the sample. Since an individual specific effect is time invariant and considered a part of the intercept, $u_{i t}$ is allowed to be correlated with other regressors (Park, 2011). The idea is that whatever effects the omitted variables have on the subject at one time, they will also have the same effect at a later time; hence their effects will be constant, or "fixed." The omitted variables must have time-invariant values with time-invariant effects, meaning that the value of the variable does not change across time (Williams, 2016). Gender, race and the educational level of the respondent's father are some examples in social studies. 
If some omitted variables are constant over time but vary between cases, and others are fixed between cases but vary over time, then both types can be included by using RE. If it is believed that the omitted variables are uncorrelated with the explanatory variables that are in the model then a RE model is probably best (Williams, 2016). Random-effects models can potentially capture unobserved heterogeneity by allowing parameters to vary across observations (Torres-Reyna, 2007).

The generally accepted way of choosing between a FE and a RE model is running a Hausman's test for each set of models where the null hypothesis is that the preferred model is RE vs. the FE (Green, 2008). It basically tests whether the unique errors $u_{i t}$ are correlated with the regressors, the null hypothesis is they are not. In the presence of such correlation, the RE slope estimator is inconsistent; consequently, the FE model should be chosen over the RE model. If there is no correlation, then both the FE and RE estimators are unbiased, but the RE estimator is preferred because it is more efficient.

Since traditional cross-section data ignore the dynamics of the changes and the heterogeneity of spatial units, this thesis is an attempt to overcome these limitations by using a spatial panel method. FE and RE regressions constitute an analytically powerful instrument to do so, which, to the authors' knowledge, has not yet been implemented in estimating the effect of RLCs on traffic collisions.

\subsubsection{Empirical Bayes Method}

The EB method is a statistical method that combines the observed collision frequency with the predicted collision frequency using safety performance functions (SPFs) to calculate the expected collision frequency for a site of interest. SPFs are regression equations that are calibrated using data from a sample of intersections, called comparison 
sites, with characteristics similar to the characteristics of the treated intersections. These characteristics typically include traffic volume and may include other variables such as traffic control and geometric characteristics. These comparable sites are used to develop an estimate of the collision frequency before the cameras were installed, which in turn is used in the calculation of the expected collision frequency (AECOM, 2014).

The number of collisions predicted from the SPF model serves as a baseline in the local environment. In the model, the predicted yearly average number of collisions is the dependent variable, while traffic volume is the independent variable. The model takes the form as specified by Hauer (1997):

$$
E(\kappa)=\beta_{0} \times V^{\beta 1}
$$

where $\mathrm{E}(\kappa)=$ mean annual expected collision frequency for the prior distribution; $V=$ annual average daily traffic (AADT); $\beta_{0}, \beta_{1}=$ model parameters. The regression parameters of the SPFs are determined by assuming that collision frequencies follow a NB distribution.

SPFs are constructed by plotting the collision and exposure data and then fitting a curve through the data using the negative binomial regression formula. The resulting curve (or equation) is the SPF. The EB method is portrayed in Figure 3.2 which illustrates how the observed collision frequency is combined with the predicted collision frequency based on the SPF model. The EB method is applied to calculate an expected collision frequency or corrected value, which lies somewhere between the observed value and the predicted value from the SPF. 


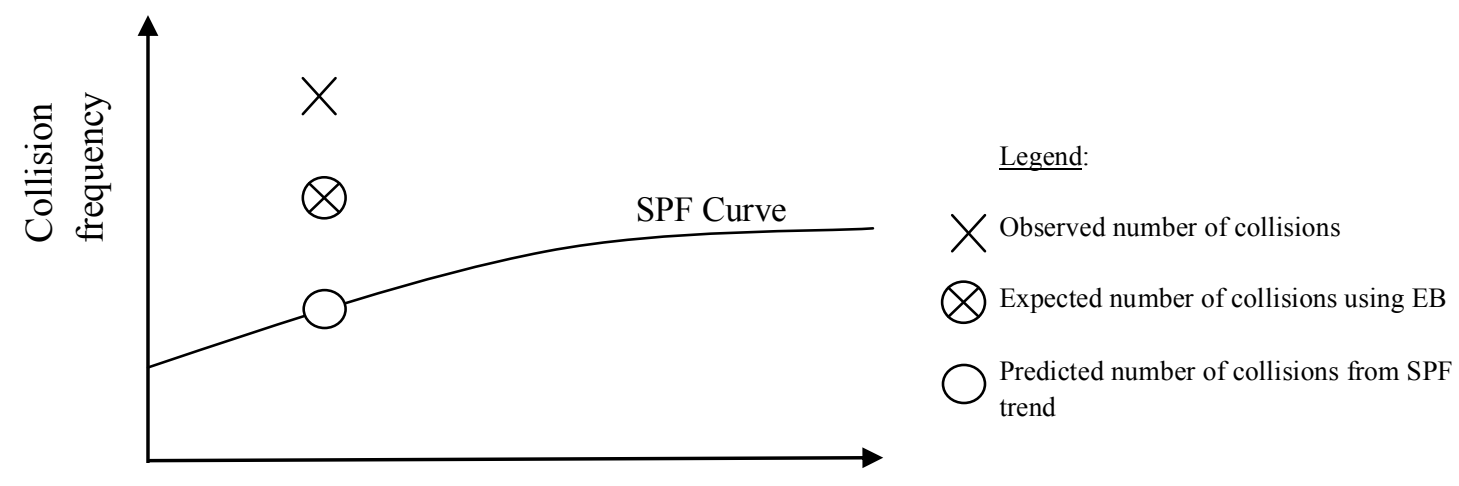

AADT

Figure 3.2 - SPF curve

The EB analysis requires AADT and collision data for the treatment site for both before and after the treatment was implemented to calculate a site's expected collision

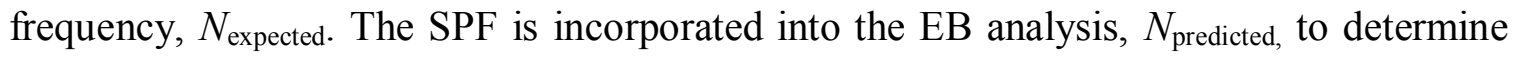
the average collision frequency at similar sites. The sites expected collision frequency can be calculated is as follows (AASHTO, 2010):

$$
N_{\text {expected }}=w \times N_{\text {predicted }}+(1-w) \times N_{\text {observed }}
$$

where

$$
w=\frac{1}{1+k \times\left(\sum_{\substack{\text { all } \\ \text { study } \\ \text { years }}}^{\text {Npredicted })}\right)}
$$

where $N_{\text {expected }}$ is an estimate of expected average collisions frequency for the study period. The $N_{\text {predicted }}$ is a predictive model estimate of average collision frequency predicted for the study period under the given conditions. The $N_{\text {observed }}$ is the observed collision frequency at the site over the study period. The $w$ is the weighted adjustment to be placed on the predictive model estimate. The $k$ is the over-dispersion parameter of the associated SPF used to estimate $N_{\text {predicted. }}$ The over-dispersion parameter is used to determine the value of a weight factor for use in the EB method. The weight factor is applied to reflect the model's statistical reliability. 
In summary, the EB method uses a reference group to estimate a SPF. The SPF is then used in combination with observed collisions pre-treatment at the treated sites to predict the number of collisions that would have been expected in the after period for the treated entities had the treatment not been implemented. This prediction is then compared to the expected number of collisions in the after period with the treatment.

\subsubsection{Case-Control Method}

Most studies in the literature review simply compared regression models to the observed results, and they stopped there. This thesis will go further into matching, which is an analytical method widely implemented in social science fields when evaluating the impact of a program. The PSM is a technique by Rosenbaum and Ruben (1983; 1985; 2002) that allows researchers to reconstruct counterfactuals using observational data by adjusting covariates between the treated and control groups. Regression analysis cannot tell researchers the distribution overlap between two samples, which is a major source of bias. Therefore, the PSM method should be used as a robust test to justify the findings estimated.

A key objective is to include variables in the models that are likely to affect both the participation and the outcome of interest so that, conditional on these measured variables, there are no unmeasured factors affecting either participation or the relevant nonparticipation outcome. These covariates are used to estimate a propensity score with a probit or logit model.

Treated units are matched to similar untreated units based on the proximity of their propensity scores. At this point, a matching algorithm has to be chosen among the 
different alternatives (e.g. nearest neighbor, radius, kernel, etc.) considering data issues (such as sample sizes) and the bias/efficiency trade-off.

Once each treated unit has been matched with one or more untreated units, the impact of the program is estimated as a weighted average of the difference in outcomes between treated and untreated. These results need to be complemented with evidence of covariate balancing between groups.

The impact of a treatment for an individual $i$, noted $\tau_{i}$, is defined as the difference between the potential outcome in case of treatment and the potential outcome in absence of treatment (Caliendo \& Kopeinig, 2005 \& Heinrich et al., 2010):

$$
\tau_{i}=Y_{1 i}-Y_{0 i}
$$

The fundamental evaluation problem arises because only one of the potential outcomes is observed for each individual $i$. The unobserved outcome is called the counterfactual outcome. Hence, estimating the individual treatment effects, $\tau_{i}$, is not possible. An evaluation seeks to estimate the mean impact of the treatment by measuring the impact of the treatment on the population and on those individuals who participated. These two parameters are known as Average Treatment Effect (ATE) and Average Treatment Effect on the Treated (ATT), respectively.

The ATE can be defined as the average effect that would be observed if everyone in the treated and the control groups received treatment, compared with if no one in both groups received treatment (Harder et al., 2010). In contrast to the ATE, the ATT refers to the average difference that would be found if everyone in the treated group received treatment compared with if none of these individuals in the treated group received treatment. 
The parameter that will be discussed here is the Average Treatment Effect on the Treated (ATT), which is defined as (Caliendo \& Kopeinig, 2005 \& Heinrich et al., 2010):

$$
\tau_{A T T}=E\left[Y_{1} \mid D=1\right]-E\left[Y_{0} \mid D=1\right]
$$

where $E$ represents the expected value and $D$ is the indicator of exposure to treatment. The second term, $E\left(Y_{0} \mid D=1\right)$, is the average outcome that the treated individuals would have obtained in absence of treatment, which is not observed. On the other hand, the term $E\left[Y_{0} \mid D=0\right]$, the value of $Y_{0}$ for the untreated individuals, is observed. However, using the mean outcome of untreated individuals $E\left[Y_{0} \mid D=0\right]$ in non-experimental studies is usually not a good idea, because it is most likely that components which determine the treatment decision also determine the outcome variable of interest. Thus, the outcomes of individuals from treatment and comparison group would differ even in the absence of treatment leading to a selection bias. The selection bias is the difference between the counterfactual for treated individuals and the observed outcome for the untreated individuals. For ATT it can be noted as (Caliendo \& Kopeinig, 2005):

$$
E\left[Y_{1} \mid D=1\right]-E\left[Y_{0} \mid D=0\right]=\tau_{A T T}+\overbrace{E\left[Y_{0} \mid D=1\right]-E\left[Y_{0} \mid D=0\right]}^{\text {selection bias }}
$$

The difference between the left hand side of equation (3.5) and $\tau_{A T T}$ is the selection bias. If this term is equal to 0 , then the $\tau_{A T T}$ can be estimated by the difference between the mean observed outcomes for treated and untreated (Heinrich et al., 2010):

$$
\tau_{A T T}=E(Y \mid D=1)-E(Y \mid D=0)
$$

The conditional probability of an entity receiving treatment is given by the covariates $(X)$ and outcomes $(Y)$. When the treatment assignment mechanism is 
unconfounded, the propensity score $(\hat{p})$ is represented as shown in the following equation (Sasidharan \& Donnell, 2013):

$$
\widehat{p}=E(D=1 \mid X)
$$

where $D=$ treatment status $(D=1$, treated and $D=0$, untreated $) ; X=$ covariates influencing treatment selection. The $\hat{p}$ is unknown in observational studies and must be estimated from the sample based on important covariates influencing the treatment selection. The $\hat{p}$ is commonly estimated using logistic regression. The propensity scores help to balance the covariates in the treated and untreated groups when the data are nonrandomized. Given a population of units denoted by $i$, if the propensity score $\hat{p}_{i}$ is known, then the ATT can be estimated as follows (Sasidharan \& Donnell, 2013):

$$
\mathrm{ATT}=E\left[E\left(Y_{1 \mathrm{i}} \mid D_{\mathrm{i}}=1, \widehat{p}_{i}\right)-E\left(Y_{0 \mathrm{i}} \mid D_{\mathrm{i}}=0, \widehat{p}_{i}\right)\right.
$$

where the outer expectation is over the distribution of $\left(\widehat{p}_{i} \mid D_{\mathrm{i}}=1\right)$ and $Y_{I i}$ and $Y_{0 i}$ are the potential outcomes in the two counterfactual situations of treatment and no treatment. The ATT is the effect of the treatment on the outcome among those who received the treatment. The treatment effect on the outcome is estimated using the propensity score matching command teffects in StataSE software.

The treatment-effects estimator that will be used in this thesis is the inverseprobability-weighted regression adjustment (IPWRA). IPWRA estimator models both the outcome and the treatment to account for the non-random treatment assignment. The covariates in the outcome model and the treatment model do not have to be the same, and they often are not because the variables that influence a subject's selection of treatment group are often different from the variables associated with the outcome. IPWRA estimators use weighted regression coefficients to compute averages of treatment-level 
predicted outcomes, where the weights are the estimated inverse probabilities of treatment (Huber, 2015).

In the first step, the probability of treatment is estimated, and in the second step regression methods are used, with weights provided by the inverse of the probability of treatment (Wooldridge, 2010). The IPWRA is called double-robust estimator as it allows for potential misspecifications in either the propensity model or the regression model predicting the two potential outcomes (Wooldridge, 2007, 2010). If one of the two models is specified incorrectly, the IPWRA estimator still produces a consistent estimate of the treatment effect (Bördős et al., 2015).

An essential aim of the analysis phases of this method is to prevent, reduce, and assess bias, confounding, and chance by matching so that a causal unbiased association between exposure and outcome is estimated (Jespen et al., 2004).

\subsubsection{Incorporating Interaction Terms}

The way interaction terms work is by running a regression analysis first, including both independent variables (an independent variable and moderator) and their interaction (product) term (Dawson, 2015). The product term should be significant in the regression equation in order for the interaction to be interpretable. The interpretation of an interaction is the change in the slope of one variable as the other variable itself changes. Using the general functional form of Eq.3.1, the conditional expected value of $y$ is a general function of the linear index function (Karaca-Mandic et al., 2012):

$$
E\left[y \mid x_{1} \mathrm{x}_{2}\right]=\exp \left(\beta_{0}+\beta_{1} \mathrm{x}_{1}+\beta_{2} \mathrm{x}_{2}+\beta_{12}\left(\mathrm{x}_{1} \mathrm{x}_{2}\right)\right)
$$

where $y$ is an outcome (dependent) variable, $x_{1}$ and $x_{2}$ are explanatory variable, and $\beta_{\mathrm{x}}$ are

parameters to be estimated. The interaction term is the product of $x_{1}$ and $x_{2}$. The effect of 
a one-unit change on the dependent variable is the marginal effect of the explanatory variable on the dependent variable. The marginal effect of an independent variable is the derivative (slope) of a given function of the covariates and coefficients of the preceding estimation (Data Analysis and Statistical Software, 2015). The derivative is evaluated at the values of the independent variables specified; if no values are specified, it is evaluated at the default values, which are the means of the independent variables.

Results can often be made more tangible by computing predicted or expected values for hypothetical cases. Such predictions are sometimes referred to as margins, predictive margins, or (StataSE 's preferred terminology) adjusted predictions (Williams, 2012). StataSE has a number of handy commands such as margins and marginsplot for making sense of regression results. A margin is a statistic computed from predictions from a model while manipulating the values of the covariates. It is typically used to estimate cell means of an effect (often an interaction), averaged over the other covariates in the population (IDRE, 2017).

These commands estimate and graphically plot predicted probabilities and are potentially more informative than regression tables when examining interaction effects, or any model which returns non-intuitive coefficients. Despite their advantages, these commands are still somewhat underutilized. It may be the case that since margins is only a few years young, many researchers simply don't know about it or don't fully understand how useful it can be at clarifying regression results. Therefore, the margins command will be used in StataSE to calculate the predicted collision counts for models with interaction terms, holding all other variables in the model at their mean values. 
In summary, the motivation for including an interaction is to get a better idea of how the RLC treatment effect varies with different intersection elements. Also, one of the motivations for investigating interactions is to identify which subgroups would benefit most from RLC treatment when resources are limited.

\subsection{Spillover Effect Method}

The distance-halo and a time-halo will be investigated to examine the local and widespread effects of the RLC enforcement. In order to assess the distance-halo, the Geographic Information System (GIS) ArcMAP 10.4.1 software was used to determine the distances of the three closest RLC intersections (referred to as "facilities" in the GIS software) to each of the non-RLC intersections (referred to as "incidents" in the GIS software). This will determine if the proximity of RLCs impact the traffic safety effectiveness; i.e. whether or not when there are two or more RLCs within a specified distance the collisions reductions were higher. The geo-processing tool utilized to accomplish this was the Network Analyst, using the closest facility tool. The closest facility solver finds and displays the shortest routes between incidents and facilities. Figure 3.3 presents an overview of the routes created, with a close up of one of the incidents and the three routes to the closest three facilities.

The Network Analyst can find multiple closest facilities from an incident by setting the "TargetFacilityCount" under the "Load Locations" of the incidents to the number of facilities wanting to search, three in this research. An example is highlighted in cyan in Figure 3.4 where the three closest facilities were ordered based on shortest travel distance to the incidents. The yellow road highlighted in each figure is the selected route which has the travel distance displayed in the table. The three closest RLC 


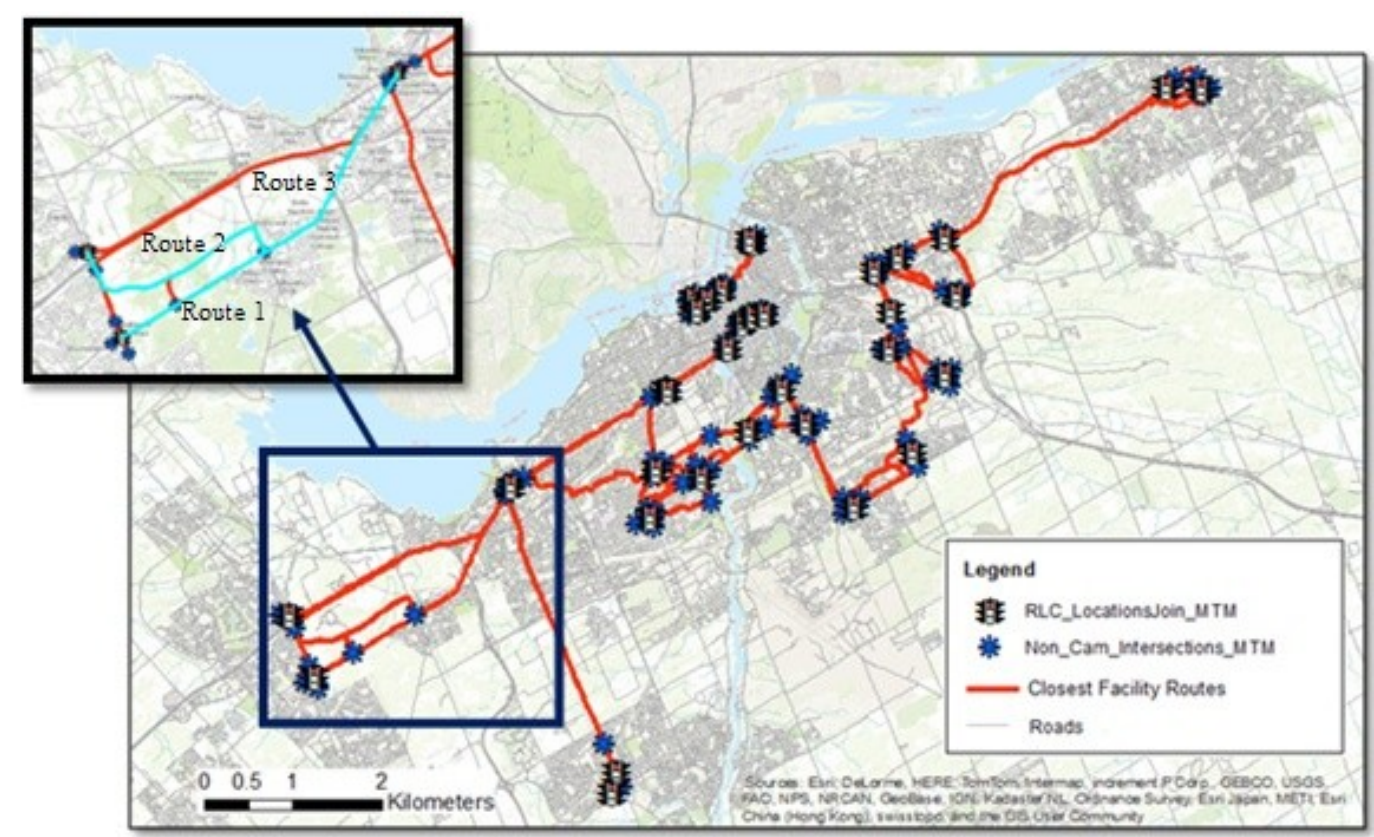

Figure 3.3 - Identifying the three closest facilities to each incident

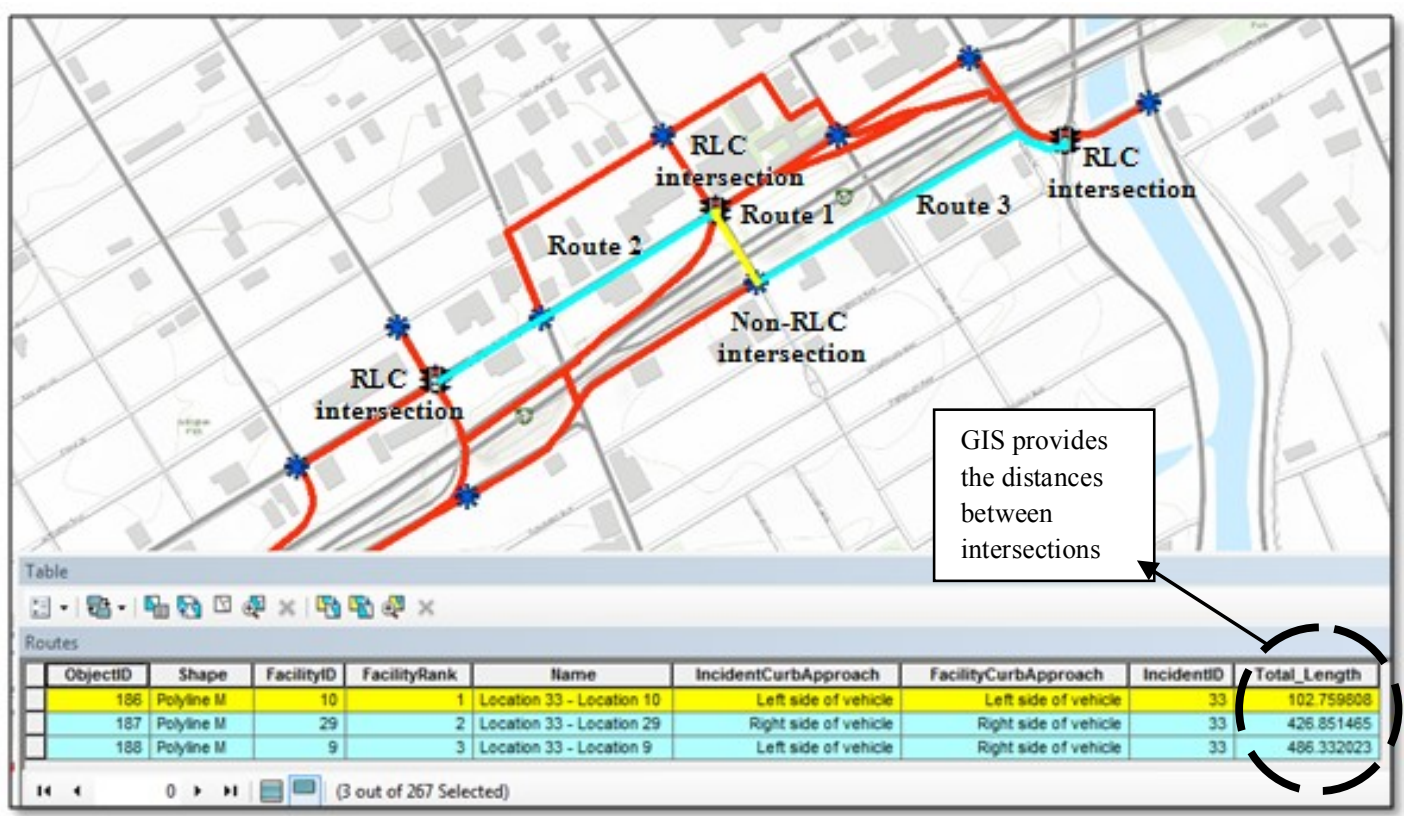

Figure 3.4 - Example of closest facilities identified

intersections to each RLC intersection were also identified. A summary of the descriptive statistics of the time and distance variables created are discussed further in Chapter 4. 


\subsection{Economic Analysis Method}

The general analysis methodology used to define the economic effects of the RLC program is evaluating the difference between the net economic costs expected without treatment and observed with treatment in the post-treatment period. The objective of a benefit-cost analysis is to estimate the net impact of a policy. The benefit-cost analysis involves the cost of the RLCs in terms capital costs, maintenance, operations, processing, etc. and the cost savings from a reduction in the number of collisions following installation of the RLCs. Two indicators of the economic worth of the RLCs are the net present value (NPV) and the benefit-cost ratio (BCR) (Chen et al., 2012). A summary of the economic analysis work flow is presented in Figure 3.5.

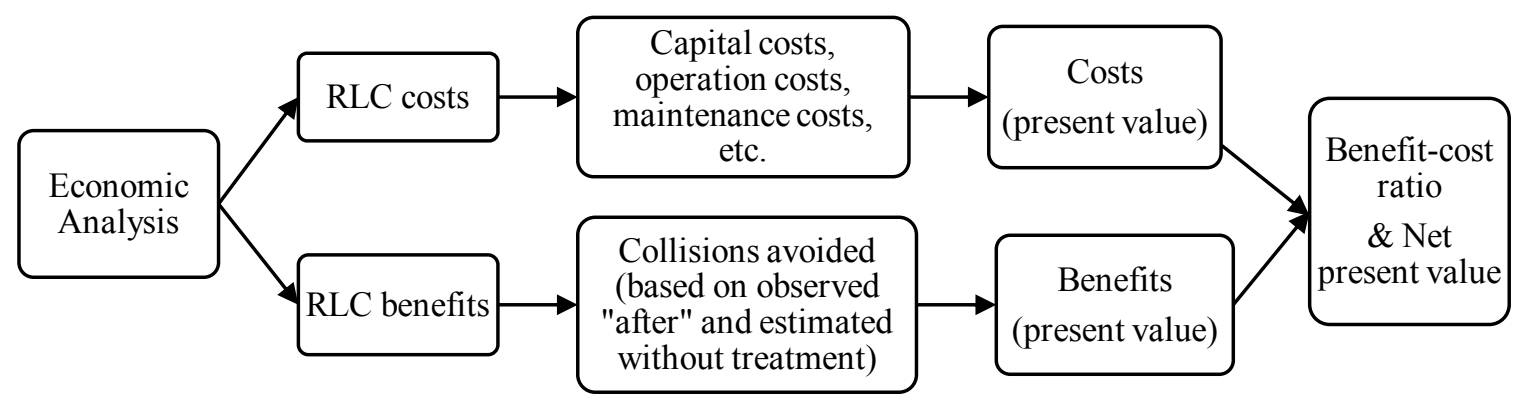

Figure 3.5 - Economical analysis process

When assessing the costs and benefits of a project, it is necessary to take into account the time value of money by converting the costs and benefits that take place in different years into a common year (Chen et al., 2012). This process is known as discounting. Discounting converts future costs and benefits that occur in different years into a value for a common year (present value) in order to make a comparison.

Since the period in which the RLCs have been in effect at various intersections is different, and also for consistency purposes, all cost amounts will be converted into present value and then compared among intersections on an average annual basis (i.e, 
annual net revenue). The future stream of discounted costs is subtracted from the future stream of discounted benefits to estimate the Net Present Value (NPV). The formulas for calculating the NPV is as follows (Chen et al., 2012):

$$
\mathrm{NPV}=\sum_{i=0}^{n}\left(\frac{B_{i}}{(1+r)^{i}}\right)-\sum_{i=0}^{n}\left(\frac{C_{i}}{(1+r)^{i}}\right)
$$

where $B_{i}=$ benefits in year $i$ resulting from savings in road collision costs, $C_{i}=$ costs of installing the RLC treatments in year 0 and the operating and maintenance costs in subsequent years $r=$ discount rate $(5 \%$ used in the analysis, consistent with the discount being used by Ministry of Transportation for Ontario (MTO) to evaluate transit projects (MKI, 2011)) and $n=$ the expected life of the project ( 5 years will be used for the RLC program in Ottawa). The duration of project life was chosen based on the 5 year contract with the RLC vendor.

The BCR ratio is calculated by dividing the total benefit amount by the total cost amount (both in NPV terms) for the entire study period. The formula for calculating the BCR is as follows (Chen et al., 2012):

$$
\mathrm{BCR}=\left[\sum_{i=0}^{n}\left(\frac{B_{i}}{(1+r)^{i}}\right)\right] /\left[\sum_{i=0}^{n}\left(\frac{C_{i}}{(1+r)^{i}}\right)\right]
$$

The BCR has no units, since it is a ratio of monetary values. A BCR significantly greater than one indicates a project is worthwhile, or if the economic worth of two or more projects are being compared then the project with the highest $\mathrm{BCR}$ is the most worthwhile. 


\section{Data Collection and Processing}

\subsection{RLC Data}

There are 34 intersections equipped with RLC units at intersections in the City of Ottawa.

A list of the RLC intersections along with their installation date and on which approach they are located is found in Table 4.1 A list of the non-RLC locations chosen is located in Appendix A (Table A).

Table 4.1 - RLC intersections in Ottawa

\begin{tabular}{|c|c|c|c|}
\hline No. & RLC Intersection Name & RLC Approach & Installation Date \\
\hline 1 & BRONSON AVE \& CARLING AVE & SB & 20-Nov-00 \\
\hline 2 & CARLING AVE \& RICHMOND RD & WB & 01-Dec-00 \\
\hline 3 & COVENTRY RD \& ST. LAURENT BLVD & NB & 01-Feb-01 \\
\hline 4 & EAGLESON RD \& HAZELDEAN RD & SB & 01-Apr-01 \\
\hline 5 & HERON RD \& RIVERSIDE DR & EB & 01-Jul-01 \\
\hline 6 & MEADOWLANDS DR \& MERIVALE RD & NB & 01-Dec-01 \\
\hline 7 & ALBERT ST \& KENT ST (OTTAWA) & NB & 01-Dec-01 \\
\hline 8 & BANK ST \& HUNT CLUB RD & EB & 13-Jun-08 \\
\hline 9 & BANK ST \& RIVERSIDE DR N & WB & 13-Jun-08 \\
\hline 10 & BASELINE RD \& MERIVALE RD & WB & 13-Jun-08 \\
\hline 11 & BELFAST RD \& ST. LAURENT BLVD & EB & 13-Jun-08 \\
\hline 12 & BOOTH ST \& OTTAWA RIVER PKWY & EB & 13-Jun-08 \\
\hline 13 & CAMPEAU DR \& MARCH RD & WB & 13-Jun-08 \\
\hline 14 & CARLING AVE \& ISLAND PARK DR & WB & 13-Jun-08 \\
\hline 15 & CATHERINE ST \& HWY 417 O'CONN & SB & 13-Jun-08 \\
\hline 16 & CATHERINE ST \& KENT ST & NB & 13-Jun-08 \\
\hline 17 & CHARLEMAGNE BLVD N \& TENTH LINE RD & SB & 13-Jun-08 \\
\hline 18 & RUSSELL RD \& WALKLEY RD & EB & 30-Sep-08 \\
\hline 19 & ALBERT ST \& BOOTH ST & WB & 07-Sep-10 \\
\hline 20 & AVIATION PKWY \& OGILVIE RD & SB & 07-Sep-10 \\
\hline 21 & BANK ST \& HERON RD & EB & 07-Sep-10 \\
\hline 22 & BERRIGAN DR \& GREENBANK RD & NB & 07-Sep-10 \\
\hline 23 & BLAIR RD \& BLAIR RD RAMP 36 & WB & 07-Sep-10 \\
\hline 24 & BRONSON AVE \& COMMISSIONER ST & SB & 07-Sep-10 \\
\hline 25 & CONROY RD \& LORRY GREENBERG DR & SB & 07-Sep-10 \\
\hline 26 & CYRVILLE RD \& INNES RD & SB & 07-Sep-10 \\
\hline 27 & DRIVEWAY (THE) \& ISLAND PARK DR & WB & 07-Sep-10 \\
\hline 28 & DUFORD DR \& ST. JOSEPH BLVD & EB & 07-Sep-10 \\
\hline 29 & FISHER AVE \& MEADOWLANDS DR & SB & 07-Sep-10 \\
\hline 30 & HAWTHORNE AVE \& QUEEN ELIZABETH DRWY & EB & 07-Sep-10 \\
\hline 31 & JOCKVALE RD \& STRANDHERD DR & SB & 07-Sep-10 \\
\hline 32 & KING EDWARD AVE \& ST. ANDREW ST & SB & $01-$ Feb-11 \\
\hline 33 & KING EDWARD AVE \& ST. PATRICK ST & EB & $01-$ Feb-11 \\
\hline 34 & SAUNDERSON DR \& SMYTH RD & NB & 01-Feb-11 \\
\hline
\end{tabular}


A map of the locations of the RLC and non-RLC intersections, illustrated in Figure 4.1, was created using the geographic information system (GIS) software ArcMap 10.4.1. The Ontario roads network layer was obtained from the Carleton University Library database GeoPortal Scholars, which included attributes about each road. The majority of the 34 RLC intersections are located in the urban areas, with a few in the suburbs.

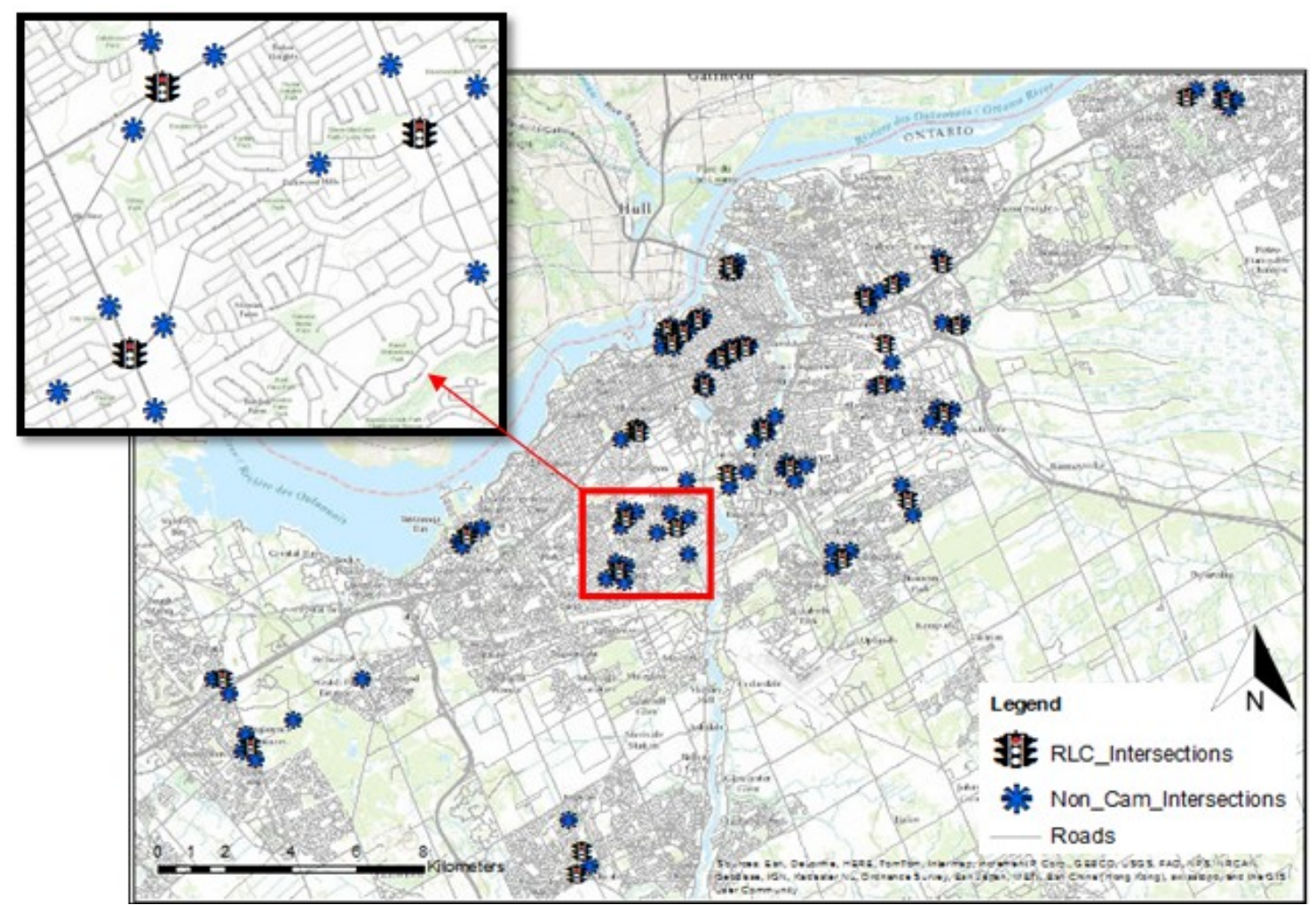

Figure 4.1 - Location of the 34 RLC intersections and comparison intersections in Ottawa

Photos of a typical RLC set up in Ottawa, taken at the Meadowlands Drive and Merivale Road intersection, are presented in Figure 4.2. The photos exhibit the camera units and their location relative to the intersection. Figure 4.3 shows a posted warning sign for RLC enforcement, which at this specific intersection was placed approximately $80 \mathrm{~m}$ away in the direction of travel from the RLC box and $125 \mathrm{~m}$ away from the midblock 


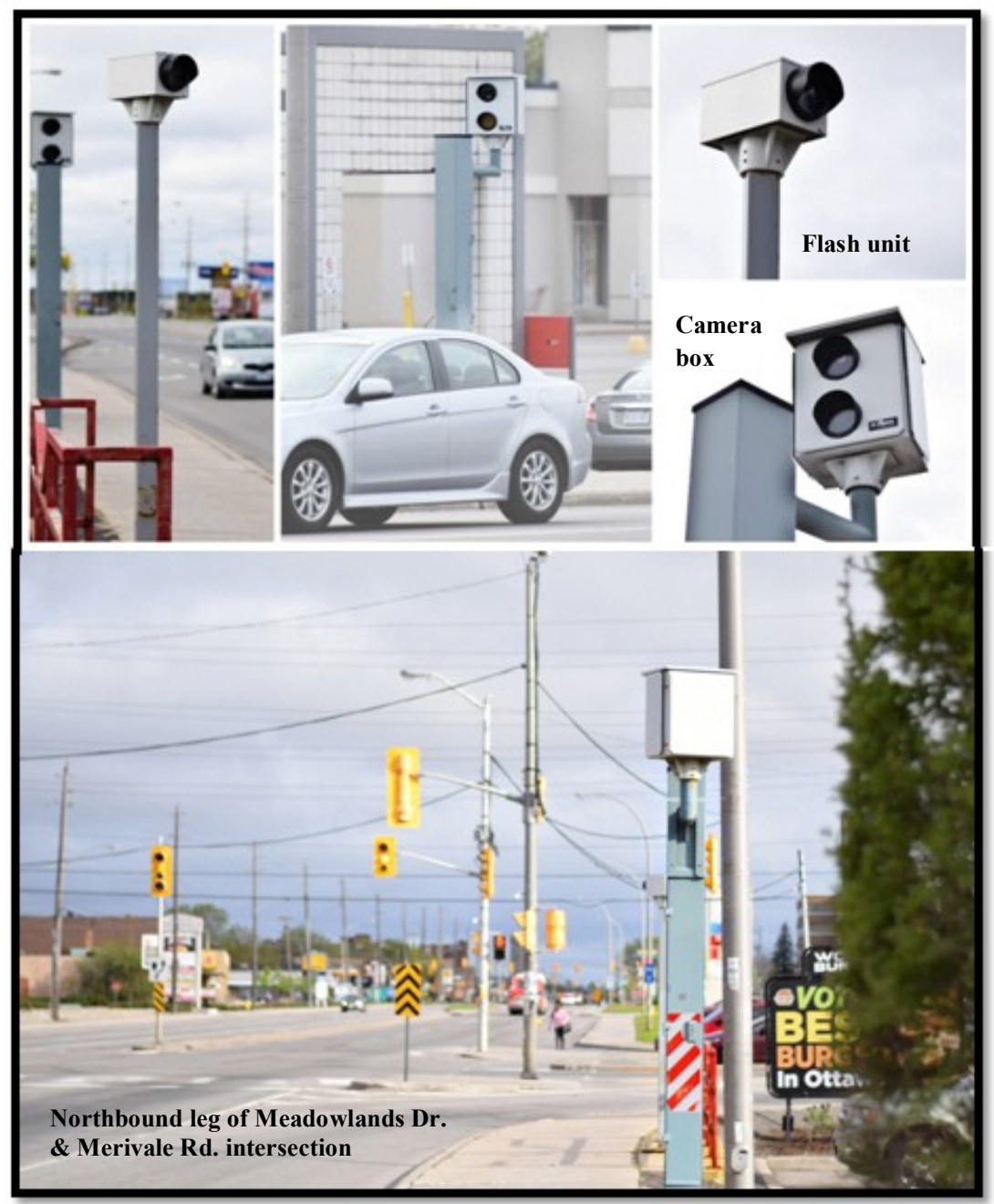

Figure 4.2 - A typical RLC set up in Ottawa (photos by: Amal Abdulsalam)

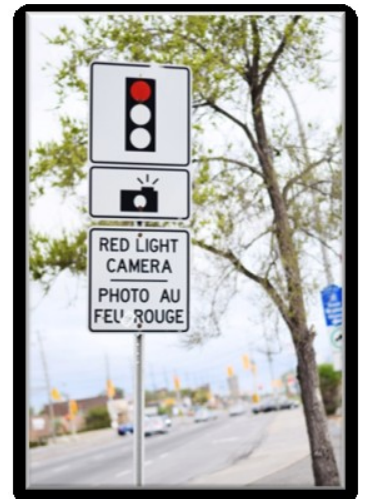

Figure 4.3 - A typical warning sign of an approaching RLC intersection (photo by: Amal Abdulsalam) 
of the intersection. The advance distance at which these signs should be located is generally dependent on the speed limit and stopping sight distances.

\subsection{Collision Data}

The City of Ottawa provided detailed traffic collision data for 34 RLC intersections and 14 non-RLC intersections for a period from January 1, 1999 to December 31, 2012. Data for the year 2013 was provided for RLC intersections installed in 2010 and 2011 to have at least three years of data for the post-treatment period. The 14 non-RLC intersections were chosen from a pool of 82 intersections based on an average total number of collisions of over 10, which is an overall average just under the RLC intersections' average. The chosen intersections have similar total collision frequencies as the RLC intersections. This selection process represents a preliminary attempt to control for selection bias by using data from non-RLC intersections that on one key variable (i.e. average number of annual collisions) are similar to the RLC intersections. Additional supplementary data collected from City of Ottawa's Annual Road Safety Reports on population demographics, number of licensed drivers, and number of vehicle registrations are presented in Appendix A (Table B) for future city-to-city comparison purposes (City of Ottawa, 2017).

The statistical spreadsheet software Microsoft Excel 2007 was used to manage data and export a dataset for the statistical analysis software StataSE 14.0. The collision database consisted of 10,267 records, of which 7,441 occurred at RLC intersections. Of the 10,267 collision cases (48 intersections), 10 were classified as fatal $(0.1 \%$ of all collisions), 2,585 were classified as serious injury (25.2\% of all collisions) and 7,671 as property-damage only (PDO) (74.7\% of all collisions), as listed in Table 4.2. 
Table 4.2 - Summary of collision database by classification and impact type for RLC and non-RLC intersections

\begin{tabular}{|c|r|r|r|r|r|r|r|r|r|}
\hline $\begin{array}{c}\text { Collision } \\
\text { Classification }\end{array}$ & \multicolumn{1}{|c|}{ Angle } & $\begin{array}{c}\text { App- } \\
\text { roa- } \\
\text { ching }\end{array}$ & $\begin{array}{c}\text { Rear- } \\
\text { end }\end{array}$ & \multicolumn{1}{c|}{$\begin{array}{c}\text { Side- } \\
\text { swipe }\end{array}$} & $\begin{array}{c}\text { Single } \\
\text { vehicle }\end{array}$ & $\begin{array}{c}\text { Turning } \\
\text { move- } \\
\text { ment }\end{array}$ & $\begin{array}{c}\text { Other/ } \\
\text { unknown }\end{array}$ & $\begin{array}{c}\text { Grand } \\
\text { Total }\end{array}$ & Percent \\
\hline Fatal injury & 8 & - & - & - & - & 2 & - & 10 & $0.10 \%$ \\
\hline $\begin{array}{c}\text { Non-fatal } \\
\text { injury }\end{array}$ & 712 & 7 & 977 & 60 & 216 & 602 & 12 & 2,586 & $25.19 \%$ \\
\hline PDO & 1,434 & 16 & 3,359 & 1,023 & 320 & 1,358 & 161 & 7,671 & $74.72 \%$ \\
\hline $\begin{array}{c}\text { Grand } \\
\text { Total }\end{array}$ & $\mathbf{2 , 1 5 4}$ & $\mathbf{2 3}$ & $\mathbf{4 , 3 3 6}$ & $\mathbf{1 , 0 8 3}$ & $\mathbf{5 3 6}$ & $\mathbf{1 , 9 6 2}$ & $\mathbf{1 7 3}$ & $\mathbf{1 0 , 2 6 7}$ & $100 \%$ \\
\hline
\end{tabular}

The traffic collision data collected reflected a period before and after the RLCs were installed. Previous work found that at least three, and preferably five years of data is the preferred before and after time period to smooth out any random fluctuations, as well as providing sufficient evidence of any trend or change in an established pattern of collisions (Chen et al., 2012).

A cumulative graph of the collisions by impact types which occurred during the study period was created in Figure 4.4. The corresponding table of the calculations is found in Appendix A (Table C). The graph identifies the most prevalent collision impact types per intersection per year being the rear-end, angle and the turning movement collisions. Not surprisingly, these three collision impact types are also part of Ottawa's RLC site selection criteria (presented earlier in Table 2.1). Sideswipe collisions account for the fourth highest impact type and accounts for over $10 \%$ of the total collisions so it will be investigated further. Therefore, this thesis will focus on these four impact types (representing nearly $93 \%$ of all collisions) in the statistical analysis performed in the next chapters.

Of the 7,441 collisions at the 34 RLC intersections, 8 were classified as fatal ( $0.11 \%$ of all collisions), 1,844 were classified as serious injury ( $24.8 \%$ of all collisions) 


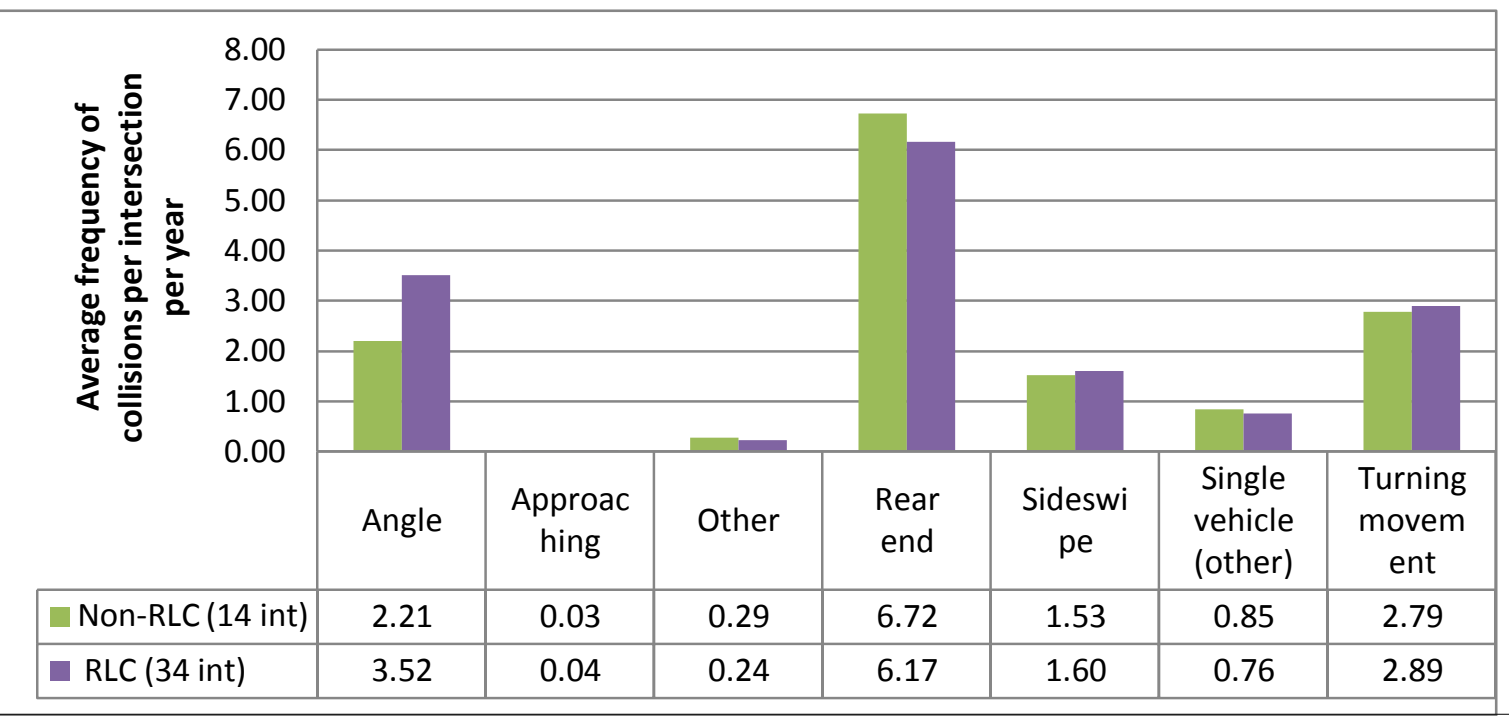

Figure 4.4 - Common collision impact types in Ottawa, ON

and 5,589 as property-damage only (PDO) (75.1\% of all collisions). The number of collisions was converted to an average annual number of collisions per intersection for the periods before and after the installation of RLCs. Results for the RLC and non-RLC intersections are presented in Table 4.3.

Table 4.3 - Average number of collisions (per intersection per year) by classification and type for RLC \& Non-RLC intersections

\begin{tabular}{|c|c|c|c|c|c|c|c|c|c|c|}
\hline \multirow{3}{*}{$\begin{array}{c}\text { Collision } \\
\text { Impact } \\
\text { Type }\end{array}$} & \multicolumn{10}{|c|}{ RLC INTERSECTIONS } \\
\hline & \multicolumn{2}{|c|}{ Total collisions } & \multicolumn{2}{|c|}{ Rear-end } & \multicolumn{2}{|c|}{ Angle } & \multicolumn{2}{|c|}{$\begin{array}{c}\text { Turning } \\
\text { movement }\end{array}$} & \multicolumn{2}{|c|}{ Sideswipe } \\
\hline & Before & After & Before & After & Before & After & Before & After & Before & After \\
\hline Total & 14.18 & 16.72 & 5.00 & 7.88 & 3.82 & 3.08 & 3.03 & 2.69 & 1.35 & 1.96 \\
\hline Fatal & 0.0172 & 0.0151 & & - & 0.0138 & 0.0151 & 0.0034 & - & - & \\
\hline Injury & 3.59 & 4.04 & 1.14 & 1.73 & 1.24 & 1.01 & 0.83 & 0.83 & 0.09 & 0.10 \\
\hline PDO & 10.58 & 12.67 & 3.86 & 6.15 & 2.57 & 2.06 & 2.20 & 1.86 & 1.27 & 1.87 \\
\hline \multirow{2}{*}{$\begin{array}{c}\text { Collision } \\
\text { Impact } \\
\text { Type } \\
\end{array}$} & \multicolumn{10}{|c|}{ NON-RLC INTERSECTIONS } \\
\hline & \multicolumn{2}{|c|}{ Total collisions } & \multicolumn{2}{|c|}{ Rear-end } & \multicolumn{2}{|c|}{ Angle } & \multicolumn{2}{|c|}{$\begin{array}{c}\text { Turning } \\
\text { movement }\end{array}$} & \multicolumn{2}{|c|}{ Sideswipe } \\
\hline Total & & 14.42 & & 6.72 & & 2.21 & & 2.79 & & 1.53 \\
\hline Fatal & & 0.0102 & & - & & 0.0051 & & .0051 & & 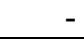 \\
\hline Injury & & 3.79 & & 1.54 & & 0.78 & & 0.99 & & 0.08 \\
\hline PDO & & 10.62 & & 5.18 & & 1.43 & & 1.79 & & 1.45 \\
\hline
\end{tabular}

As can be seen from the results, the chosen non-RLC intersections have similar frequencies to the RLC intersections to be comparable when analyzing in the next 
chapters. Detailed counts of the number of before and after collisions per intersection for both the RLC intersections and non-RLC intersections can be found in Appendix A.

Two sample t-tests were performed as simple, initial tests to determine if there was a statistically significant change in the mean of collisions between RLC intersections and non-RLC intersections chosen as the control sites. The two-sample t-test still works when the assumption of normality is violated, but only if the underlying distribution is not highly skewed (Minitab Statistical Software, 2017). A $p$-value of 0.05 or less indicates that any observed differences are unlikely to be due to random variation alone (Kloeden et al., 2009). The full output of the results is presented in Appendix A. A summary of the results is as follows:

- The total collisions' t-test was statistically significant concluding that collision frequencies differed and are unlikely due to random variation alone (an increase from 14.18 to 16.72$)$.

- The traffic volumes' t-test concluded that the before and after differed (an increase in traffic volume which is probably trend related, from 10.31 to 10.48 ; this could mean that accident frequencies caused by RLC installation will be slightly overstated).

- The rear-end collisions' t-test was statistically significant concluding that collision frequencies differed and are unlikely due to random variation alone (an increase from 5.00 to 7.88 ).

- The angle collisions' t-test was statistically significant concluding that collision frequencies differed and are unlikely due to random variation alone (a decrease from 3.82 to 3.08 ). 
- The turning movement collisions' t-test was not statistically significant concluding that collision frequencies do not differ significantly and is likely due to random variation (turning movement collisions decrease marginally from 3.03 to 2.69 but not significant ).

- The sideswipe collisions' t-test was statistically significant concluding that collision frequencies differed and are unlikely due to random variation alone (an increase from 1.35 to 1.96$)$.

However, the standard t-test does not consider relationships among the coefficients. Therefore, further statistical tests will be carried out and presented in Chapter 5.

Only one public report was found, published in 2012 by the City of Ottawa, assessing the overall RLCs performance in the city (Keefe, 2012). The report simply conducted a naïve before-after study and based their policy decisions on those estimations. A naïve before-after study is a simple before-after comparison where the safety effect of a treatment is assessed by directly comparing the collision frequency in the before period with the collision frequency in the after period (Hauer, 1997). This is also referred to as a trend analysis. A summary of results from the City of Ottawa report are presented in Table 4.4. The actual number of collisions used in the counts when calculating the percent difference was not mentioned in the report and number of intersections used in the counts was not mentioned for each year.

Table 4.4 - RLC effect in Ottawa - adapted from Keefe (2012) report

\begin{tabular}{|l|c|c|c|c|}
\hline \multicolumn{1}{|c|}{ Collision Impact Type } & $\mathbf{1}$ yr after & 3 yrs after & 5yrs after & 10 yrs after \\
\hline \hline Total & $-2 \%$ & $+3 \%$ & $-8 \%$ & $-12 \%$ \\
\hline Angle & $-3 \%$ & $-12 \%$ & $-33 \%$ & $-29 \%$ \\
\hline Rear-end & $+1 \%$ & $+11 \%$ & $+8 \%$ & $+20 \%$ \\
\hline
\end{tabular}


A comparison set of naïve before-after estimates was conducted using the set of data of the RLC intersections from this thesis, presented in Table 4.5. The way the number of collisions was counted was from the exact date each RLC was installed until a year after, which is more accurate than by a simple fiscal year count.

\section{Table 4.5 - RLC effect in Ottawa from database}

\begin{tabular}{|c|c|c|c|c|c|c|c|c|c|c|c|c|}
\hline \multirow{3}{*}{$\begin{array}{c}\text { Collision } \\
\text { Impact } \\
\text { Type }\end{array}$} & \multicolumn{3}{|c|}{ (34 intersections) } & \multicolumn{3}{|c|}{ (31 intersections) } & \multicolumn{3}{|c|}{ (18 intersections) } & \multicolumn{3}{|c|}{ (7 intersections) } \\
\hline & \multicolumn{2}{|c|}{$\begin{array}{l}\text { average \# of } \\
\text { collisions }\end{array}$} & \multirow{2}{*}{$\begin{array}{c}\% \\
\text { change* }\end{array}$} & \multicolumn{2}{|c|}{$\begin{array}{l}\text { average \# of } \\
\text { collisions }\end{array}$} & \multirow{2}{*}{$\begin{array}{c}\% \\
\text { change* }\end{array}$} & \multicolumn{2}{|c|}{$\begin{array}{l}\text { average \# of } \\
\text { collisions }\end{array}$} & \multirow{2}{*}{$\begin{array}{c}\% \\
\text { change* }\end{array}$} & \multicolumn{2}{|c|}{$\begin{array}{l}\text { average \# of } \\
\text { collisions }\end{array}$} & \multirow{2}{*}{$\begin{array}{c}\% \\
\text { change* }\end{array}$} \\
\hline & $\begin{array}{l}\text { Base- } \\
\text { year }\end{array}$ & $\begin{array}{l}1 \mathrm{yr} \\
\text { after }\end{array}$ & & $\begin{array}{l}\text { Base- } \\
\text { year }\end{array}$ & $\begin{array}{l}3 \mathrm{yrs} \\
\text { after }\end{array}$ & & $\begin{array}{l}\text { Base- } \\
\text { year }\end{array}$ & $\begin{array}{l}5 \mathrm{yrs} \\
\text { after }\end{array}$ & & $\begin{array}{l}\text { Base- } \\
\text { year }\end{array}$ & $\begin{array}{l}10 \mathrm{yrs} \\
\text { after }\end{array}$ & \\
\hline Total & 15.71 & 15.00 & $-4 \%$ & 16.52 & 15.74 & $-5 \%$ & 18.06 & 17.83 & $-1 \%$ & 17.71 & 19.39 & $9 \%$ \\
\hline Angle & 3.35 & 3.15 & $-6 \%$ & 3.58 & 3.42 & $-5 \%$ & 4.39 & 3.47 & $-21 \%$ & 4.57 & 3.61 & $-21 \%$ \\
\hline $\begin{array}{l}\text { Rear- } \\
\text { end }\end{array}$ & 6.68 & 6.38 & $-4 \%$ & 6.94 & 6.96 & $0 \%$ & 7.61 & 8.08 & $6 \%$ & 7.29 & 9.80 & $35 \%$ \\
\hline
\end{tabular}

It is important to note that the average number of collisions, i.e. for "5 years after", is the average number of collisions from year 1, 2, 3, 4 and 5 after the RLC was installed. The year before the RLC was installed at an intersection was used as the base year for all counts. In the Keefe (2012) report, the base year was the year the RLC was installed. Since the RLC locations have been in place for different lengths of time, only some of the locations can be used for the longer term evaluation. For example, all RLC locations were used to calculate the one year before and after data, but only the seven locations where the red light camera has been in place for over 10 years were used to calculate the 10 year percentage.

There are a few noted differences between the estimations conducted by City of Ottawa and from the data collected in this thesis. The main difference will be from the number of intersections used for each calculation, which was not identified in the City of 
Ottawa report. The results in Table 4.5 show that the magnitude of the effect becomes more apparent as the results of more years get analyzed. After a 10 year assessment, results indicate that the angle collisions decrease by $21 \%$ and the rear-end collisions rise by about $35 \%$, which in-turn cause the total collisions to increase by $9 \%$.

However, this naïve before-after analysis does not account for possible bias due to RTM and does not account for temporal effects or trends, which are changes taking place independent of the RLC treatment. It is not known what part of the changes can be attributed to the RLC treatment and part due to other influences. Without a control group, it is difficult to establish the cause and effect relationship between the RLC treatment and collisions as an outcome. Therefore, it is not acceptable to base decisions based on the results obtained from this method and more reliable statistical prediction methods will be assessed.

Although the collision summaries in this Section provide some insight on the data, the next step is to gather spatial and geometric data related to the intersections to better understand the trends and patterns. Knowing more about the conditions when collisions happen helps policy makers identify areas for review and create targeted approaches to reducing and ultimately preventing dangerous traffic collisions.

\subsection{Selection of Variables}

One important goal in model building is to find the best fitting model to describe the relationship between the outcome and a set of independent variables. Data for 11 independent variables (including the treatment variable) were gathered based on sitespecific local factors. Descriptive statistics of the explanatory variables are presented in Table 4.6. The sample size should be at least 10 observations per variable, since selection 
Table 4.6 - Variable definitions and summary statistics

\begin{tabular}{|c|c|c|c|c|c|}
\hline \multicolumn{6}{|c|}{ Dependent Variables } \\
\hline Description (variable name) & Observations & Mean & Std.Dev. & Min. & Max. \\
\hline Number of total collisions (totalcollisions) & 685 & 14.99 & 7.74 & 0 & 39 \\
\hline Number of angle collisions (angle) & 685 & 6.33 & 5.39 & 0 & 30 \\
\hline Number of rear-end collisions (rearend) & 685 & 3.14 & 3.00 & 0 & 24 \\
\hline $\begin{array}{l}\text { Number of turning movement collisions } \\
\text { (turningmovement) }\end{array}$ & 685 & 2.86 & 3.02 & 0 & 17 \\
\hline Number of sideswipe collisions (sideswipe) & 685 & 1.58 & 1.89 & 0 & 10 \\
\hline \multicolumn{6}{|c|}{ Independent Variables } \\
\hline Description (variable name) & Observations & Mean & Std.Dev. & Min. & Max. \\
\hline Presence of RLC (rlcinstalled2) & 685 & 0.29 & 0.45 & 0 & 1 \\
\hline Natural log of total AADT (lnaadt) & 685 & 10.42 & 0.40 & 8.96 & 11.20 \\
\hline Presence of a median (median) & 685 & 0.67 & 0.47 & 0 & 1 \\
\hline $\begin{array}{l}\begin{array}{l}\text { Presence of a right-turn channelization } \\
\text { (channelization) }\end{array} \\
\end{array}$ & 685 & 0.42 & 0.49 & 0 & 1 \\
\hline Number of thru lanes (thrulanes) & 685 & 2.26 & 0.70 & 1 & 4 \\
\hline Number of left-turn lanes (leftturn) & 685 & 0.69 & 0.62 & 0 & 2 \\
\hline Fully-protected left-turn signal $(f p l t)$ & 685 & 0.37 & 0.48 & 0 & 1 \\
\hline Permitted-protected left-turn signal ( $p p l t)$ & 685 & 0.27 & 0.45 & 0 & 1 \\
\hline Permitted left-turn signal (plt) & 685 & 0.46 & 0.50 & 0 & 1 \\
\hline Posted speed limit on Road 1 (speed1) & 685 & 54.41 & 8.38 & 40 & 70 \\
\hline Posted speed limit on Road 2 (speed2) & 685 & 54.73 & 7.34 & 40 & 80 \\
\hline
\end{tabular}

bias and model instability become major issues in smaller sample sizes (Sauerbrei et al., 2007).

Drawings of the signalized intersections were not available at the City of Ottawa's traffic engineering department to record the road characteristics. Therefore, a geometry database was created manually using Google Earth images to record road characteristics of the intersections including the number of through lanes, number of left-turn lanes, presence of median, and right-turn channelization. Additional signal timings and posted speed limits of the cross roads of each studied intersection were obtained from the Traffic Operations department at the City of Ottawa. 
Average annual daily traffic (AADT) volumes were obtained from the City of Ottawa and used to attempt to adjust for exposure. The entering AADT data for all years in the study period are required in order to conduct a before-and-after study using the EB method. However, the collected data lacked some AADT values for the collected study period. As a result, the missing AADTs were estimated using an annual growth rate of $5 \%$, as used for other projects in Ottawa (Date, 2014).

Since AADT does not necessarily follow a linear distribution, a better approach was to introduce the natural $\log$ as the independent variable. Count models need some sort of mechanism to deal with the fact that counts can be made over different observation periods (IDRE, 2001). For example, the number of collisions is recorded for 50 different intersections, however, the vehicles that pass through the intersections can vary greatly. Fifteen collisions for 30,000 vehicles is very different from 15 collisions for 1,500 vehicles. Count models account for these differences by including the log of the exposure variable, which reduces the skew in the AADT data (Statistical Consulting Group , 2007).

When building regression models, the aim is identify influential variables and determine the functional form. The models are fitted using the log-link function in the StataSE Software. The first approach will be including all variables in the model (all-in model) to provide as complete control of confounding as possible within the given data set. This approach is based on the fact that it is possible for individual variables not to reveal strong confounding, but when taken collectively, substantial confounding can be present in the data set (Miettinen, 1976). 
However, it may be cautioned that an all-in model may be over-fitted and thus produce numerically unstable parameter estimates. An over-fitted model can cause the regression coefficients, $p$-values, and R-squared to be misleading (Frost, 2015). When this happens, the regression model becomes tailored to fit the flukes and random noise in the specific sample rather than reflecting the overall population. If another sample gets used, it would have its own flukes, and the original over-fitted model would not likely fit the new data. Instead, the model should approximate the true model for the entire population.

Therefore, the next step is to explore the variables manually using a stepwise backward procedure. The procedure starts from an all-in model, and variables are deleted stepwise starting with the least significant. In case a variable is significant at the $95 \%$ confidence interval $(p \leq 0.05)$, the variable is kept in the model. If the variable is not significant, the variable is omitted from the model. When this procedure is completed, any variables that have become insignificant $(p>0.05)$ throughout the process are omitted from the model. Variables that reach $p \leq 0.05$ are considered as potentially important.

The Akaike Information Criterion (AIC) will be used to compare the models. The AIC is a measure of model goodness-of-fit adjusted for the number of parameters that can be compared across models (Akaike, 1987; Washington et al., 2003). The criterion provides an estimate of the trade-off between model accuracy and complexity. The model with the lowest AIC model is considered to be the best model. The only restriction is that the traffic volume variable (lnaadt) is always added to the model, irrespective of its impact to the AIC value, because a model that does not incorporate any measure of exposure cannot be credible from a theoretical point of view (Reurings et al., 2006). 
In the context of PSM models, the aim is to find a combination of variables that gets balanced between treated and untreated subjects in the matched sample. Brookhart et al. (2006) and Pearl (2010) suggested that variables that do not affect exposure but that affect the outcome should always be included in the propensity score model. Therefore, the inclusion of covariates in the model is more liberal than in most traditional settings. For instance, covariates with $p$-values larger than 0.05 can be included in the propensity score model (D'Agostino, 2007). The inclusion of non-significant variables will not bias the estimates or make them inconsistent, it can, however, increase their variance (Caliendo \& Kopeinig, 2005). Although it is not possible to know for sure whether there is an unmeasured confounder or not, the more measured confounders that have been included, the more plausible the assumption.

Correlation of variables in the propensity score model are not considered to be a problem since the goal of the model is to balance the covariates, not to find statistically significant relationships between the covariates and the treatment (Conniffe et al., 2000). In order to have balanced data, the treatment and control groups need to look substantially similar.

\subsection{Spatial Data}

Distance variables obtained from the ArcMAP 10.4.1 software, based on the three closest RLC intersection distances, were added to the panel database. Summary statistics of those three variables (disthalo1, disthalo2, and disthalo3) are presented in Table 4.7. Additional sets of variables were also created to see if any would have an apparent trend on the number of collisions. The first set of variables consists of two variables of a binary response of whether or not the closest RLC intersection is within $0 \mathrm{~m}$ to $500 \mathrm{~m}$ or $500 \mathrm{~m}$ 
to $1000 \mathrm{~m}$. The second set of variables also consists of two variables of a binary response of whether or not there are more than two RLCs between $0 \mathrm{~m}$ to $500 \mathrm{~m}$ and between 500 $\mathrm{m}$ to $1000 \mathrm{~m}$. The last variable in Table 4.7 consists of a binary response variable determining whether or not there are three RLCs within a $1 \mathrm{~km}$ zone. These variables were created to determine if there is a significant distance where the RLC sites would spillover to non-RLC intersections.

Table 4.7 - Summary of distance halo variables

\begin{tabular}{|c|c|c|c|c|c|}
\hline Description (variable name) & Observations & Mean & Std. Dev. & Min. & Max. \\
\hline $\begin{array}{l}\text { Distance halo to closest RLC int. - } \\
\text { measure 1-(disthalo1) }\end{array}$ & 685 & 690.46 & 886.04 & 74.98 & 6117.09 \\
\hline $\begin{array}{l}\text { Distance halo to 2nd closest RLC int. } \\
\text {-measure 2- (disthalo2) }\end{array}$ & 685 & 2201.17 & 2262.52 & 127.78 & 11214.45 \\
\hline $\begin{array}{l}\text { Distance halo to 3rd closest RLC int. } \\
\text {-measure 3-(disthalo3) }\end{array}$ & 685 & 3554.78 & 3251.39 & 486.33 & 13823.69 \\
\hline $\begin{array}{l}\text { Binary where "1" if closest RLC is } \\
\text { within } 0 \text { to } 500 \mathrm{~m} \text { (closestrlc0to500) }\end{array}$ & 685 & 0.4350 & 0.4961 & 0 & 1 \\
\hline $\begin{array}{l}\text { Binary where "1" if closest RLC is } \\
\text { within } 500 \text { to } 1000 \mathrm{~m} \\
\text { (closestrlc500to1000) }\end{array}$ & 685 & 0.1679 & 0.3740 & 0 & 1 \\
\hline $\begin{array}{l}\text { Binary where "1" if two or more } \\
\text { RLCs installed within } 500-1000 \mathrm{~m} \\
\text { Binary where "1" if two or more } \\
\text { RLCs installed within } 0 \text { to } 500 \mathrm{~m} \\
\text { (twoormorerlc0to500) }\end{array}$ & 685 & 0.0628 & 0.2427 & 0 & 1 \\
\hline $\begin{array}{l}\text { Binary where "1" if two or more } \\
\text { RLCs installed within } 500 \text { to } 1000 \mathrm{~m} \\
\text { (twoormorerlc500to1000) }\end{array}$ & 685 & 0.0204 & 0.1416 & 0 & 1 \\
\hline $\begin{array}{l}\text { Binary where "1" if three RLCs are } \\
\text { installed within } 1000 \mathrm{~m} \\
\text { (Threewithin1000) }\end{array}$ & 685 & 0.0832 & 0.2764 & 0 & 1 \\
\hline
\end{tabular}

Next, time-halo variables were created and added to the database. Variables with several different timing sequences were created and examined to see if any have an effect on the frequency of collisions. A summary of the time-halo descriptive statistics are found in Table 4.8 .

The first count variable created (timehaloyr) was a simple time trend by year from installation year $(1,2,3,4$, etc.) to determine if the number of collisions change as the 
Table 4.8 - Summary of time-halo variables

\begin{tabular}{|l|r|r|r|r|r|}
\hline \multicolumn{1}{|c|}{ Description (variable name) } & Observations & \multicolumn{1}{c|}{ Mean } & \multicolumn{1}{c|}{ Std. Dev. } & \multicolumn{1}{c|}{ Min. } & Max. \\
\hline \hline Time trend (1,2,3,4..) (timehaloyr) & 685 & 6.5596 & 4.0711 & 0 & 14 \\
\hline $\begin{array}{l}\text { Cumulative count of RLCs installed } \\
\text { (numcam) }\end{array}$ & 685 & 13.4502 & 11.4363 & 0 & 34 \\
\hline $\begin{array}{l}\text { Binary variable where a "1" being a year } \\
\text { where a RLC was installed (timehalo) }\end{array}$ & 685 & 0.4252 & 0.4945 & 0 & 1 \\
\hline $\begin{array}{l}\text { Time trend that resets when new RLCs get } \\
\text { installed (timehalo2) }\end{array}$ & 685 & 2.0321 & 2.3998 & 0 & 7 \\
\hline
\end{tabular}

years pass by. This is to examine whether drivers are more aware or indifferent to the presence on RLCs with the progression of years. The second count variable created (numcams) was a cumulative count of the number of RLCs as they were installed by year to answer the question of whether the increase in number of RLCs have an impact on the frequency of collisions. A third binary variable (timehalo) was created having "1" for the years where RLCs were installed (year 2000, 2001, 2008, 2010, and 2011) and "0" for the years with no cameras installed. This variable would examine if the years the RLCs get installed are any different than other years with no new installations. The fourth count variable created (timehalo2) resets at "0" for the years that RLCs get installed and is a continuous count adding one up for every year with no new installations. These different time-halo variables will be tested to find out if the presence of RLCs is substantial in both the short and long-term, as these types of variables have not been examined before related to RLCs.

It is important to understand spillovers and the overall effect on the entire population. This analysis will examine whether the RLC treatment creates benefits to surrounding intersections or by diverting the dangers to them. It will also shed light on how intersections respond to the treatment over time. 


\subsection{Cost Data}

Two types of cost data were used in the economic evaluation of the RLCs: the RLC costs and the social costs of collisions. The RLC costs consist of the capital, maintenance and operational costs of the RLCs. The social costs of collisions are the direct and indirect social cost savings from a reduction in the number of vehicle collisions following the installations of the RLCs.

\subsubsection{RLC Costs}

Currently the RLC sites in the city of Ottawa are on a lease/operate/maintain basis from the vendor Traffipax under contract. The exact RLC vendor cost is confidential, but according to Mayor Jim Watson "the city contracts the cameras' operation and monitoring to an outside company at a cost of about \$35,000 annually" (Dodge, 2016).

This cost estimate includes the installation, operation and maintenance of RLC equipment, providing access to vehicle ownership information from MTO, and the agreement with the City of Toronto for processing infraction notices within the Red Light Camera Enforcement Program's Joint Processing Centre.

The City of Ottawa has an annual budget of $\$ 1.25 \mathrm{M}$ for its RLC program (City of Ottawa, 2016). According to Mr. Start Edison, Program Manager of Ottawa's Traffic Engineering and Control department, the total costs for the RLC program in Ottawa for 2014 was $\$ 1,189,053$ and for 2015 was $\$ 1,251,783$ (S. Edison (email communication, Mon 2016-03-14 8:32 AM)). The estimated total RLC cost for the year 2016 is $\$ 1,267,641$, based on a Consumer Price Index of 1.27\% (Statistics Canada, 2017).

The total number of fines that have been issued on a yearly basis since the program began in 2000 until 2011 were obtained from the City of Ottawa (2012) "Red 
Light Camera/Roadside Speed Indicator Update" report. For the years 2012 and 2013, an estimated average of 9,200 fines was issued each year (CTV News, 2015). Data obtained from the City of Ottawa shows that almost twice as many vehicles were caught RLR in 2014 compared to 2013. In 2014, 17,186 tickets were issued at intersections with RLCs. The spike coincides with a decision by the City to operate all 34 RLCs every day, and at the same time. Prior to 2014, the number of cameras in operation rotated from site-to-site (CTV News, 2015). In 2015, 17,658 drivers received RLR tickets. During the first three months of 2016, the City mailed 5,266 red-light camera violations (Willing, 2016). The complete number of violations is not out yet for the year 2016, therefore, the same number of tickets from 2015 will be used in the analysis for 2016 to avoid overestimation.

When the program began in 2000 , the fine amount for a RLR violation was $\$ 190$ and was then increased in January 2010. The current fine amount set by the province is $\$ 325$, including a $\$ 5$ service fee and a $\$ 60$ victim's surcharge (City of Ottawa, 2012). This results in net revenue of $\$ 260$ per red-light violation being returned to the municipality. A summary of the annual number of RLR violations, costs of violations and the annual estimated net revenues is presented in Table 4.9.

The City of Ottawa does not keep track of how many tickets are traceable to each RLC. Krista Tanaka, program manager of road safety and traffic investigations in Ottawa, said, "The City does not track tickets on an intersection-by-intersection basis because collisions are deemed to be much more important. We don't use tickets as our measure of effectiveness for the cameras" (Tumilty, 2017). 
Table 4.9 - Estimated net revenue (from fines) of the RLC program in Ottawa

\begin{tabular}{|r|r|r|r|r|r|}
\hline Year & $\begin{array}{c}\text { Number of } \\
\text { RLCs } \\
\text { installed }\end{array}$ & \multicolumn{1}{c|}{$\begin{array}{c}\text { Total Number of } \\
\text { RLR Fines } \\
\text { Issued }\end{array}$} & $\begin{array}{c}\text { Fine amount } \\
\text { per RLR } \\
\text { violation }\end{array}$ & $\begin{array}{c}\text { Net revenue } \\
\text { from fines* }\end{array}$ & $\begin{array}{c}\text { Annual net } \\
\text { estimate of RLR } \\
\text { fine revenue }\end{array}$ \\
\hline \hline $\mathbf{2 0 0 1}$ & 7 & 4,737 & $\$ 190$ & $\$ 155$ & $\$ 734,235$ \\
\hline $\mathbf{2 0 0 2}$ & 7 & 1,762 & $\$ 190$ & $\$ 155$ & $\$ 273,110$ \\
\hline $\mathbf{2 0 0 3}$ & 7 & 3,253 & $\$ 190$ & $\$ 155$ & $\$ 504,215$ \\
\hline $\mathbf{2 0 0 4}$ & 7 & 2,861 & $\$ 190$ & $\$ 155$ & $\$ 443,455$ \\
\hline $\mathbf{2 0 0 5}$ & 7 & 3,212 & $\$ 190$ & $\$ 155$ & $\$ 497,860$ \\
\hline $\mathbf{2 0 0 6}$ & 7 & 2,572 & $\$ 190$ & $\$ 155$ & $\$ 398,660$ \\
\hline $\mathbf{2 0 0 7}$ & 7 & 5,809 & $\$ 190$ & $\$ 155$ & $\$ 900,395$ \\
\hline $\mathbf{2 0 0 8}$ & 18 & 5,974 & $\$ 190$ & $\$ 155$ & $\$ 925,970$ \\
\hline $\mathbf{2 0 0 9}$ & 18 & 8,873 & $\$ 190$ & $\$ 155$ & $\$ 1,375,315$ \\
\hline $\mathbf{2 0 1 0}$ & 31 & 12,674 & $\$ 325$ & $\$ 260$ & $\$ 3,295,240$ \\
\hline $\mathbf{2 0 1 1}$ & 34 & 9,631 & $\$ 325$ & $\$ 260$ & $\$ 2,504,060$ \\
\hline $\mathbf{2 0 1 2}$ & 34 & 9,200 & $\$ 325$ & $\$ 260$ & $\$ 2,392,000$ \\
\hline $\mathbf{2 0 1 3}$ & 34 & 9,200 & $\$ 325$ & $\$ 260$ & $\$ 2,392,000$ \\
\hline $\mathbf{2 0 1 4}$ & 34 & 17,186 & $\$ 325$ & $\$ 260$ & $\$ 4,468,360$ \\
\hline $\mathbf{2 0 1 5}$ & 34 & 17,658 & $\$ 325$ & $\$ 260$ & $\$ 4,591,080$ \\
\hline $\mathbf{2 0 1 6}$ & 34 & 17,658 & $\$ 325$ & $\$ 260$ & $\$ 4,591,080$ \\
\hline$*=$ cost per fine minus the $\$ 60$ victim surcharge and minus the $\$ 5$ service fee & \\
\hline
\end{tabular}

\subsubsection{Social Costs}

Collision victims and the society as a whole are affected by traffic collisions. The social cost of traffic collisions consists of two components used to assign monetary costs, direct and indirect costs (Hanley, 2004). The first component is quite definitive direct costs like property damage, emergency response costs, and material expended in dealing with collisions. The second component is indirect costs which are very abstract and nontangible estimating the quality of life costs. Quality of life costs includes aspects like physical and mental suffering and permanent cosmetic damage (Hanley, 2004; De Leur, 2010).

The human consequences of collisions are measured by how much society is willing-to-pay to avoid these consequences (Vodden et al., 2007). These are costs that a society is willing to pay to prevent or reduce the risks associated with the occurrence of 
collisions, particularly collisions that involve injury and death (i.e. property damage costs, emergency response costs, health services costs, courts and legal costs, tow truck and traffic delay costs).

In 2007, the Transportation Association of Canada published a report entitled Analysis and Estimation of the Social Cost of Motor Vehicle Collisions in Ontario (Vodden et al., 2007) which has the most recent social costs available for Ontario. This report advanced an earlier social cost model from Simpson et al. (1994) by adding cost components related to traffic delay (i.e. extra time, fuel, and pollution). The average willingness-to-pay social cost is estimated at $\$ 15,700,000$ per fatal collision, $\$ 82,000$ per injury collision, and $\$ 8,000$ per PDO collision (2004 CAD). The details of cost allocation based on the different components are found in Appendix B, adapted from Vodden et al. (2007).

Since these values are from 2004, the Consumer Price Index (inflation rates) from 2004 to 2016 were obtained from Statistics Canada (2017) to adjust the costs. The estimated 2016 average willingness-to-pay social cost is estimated at $\$ 19,500,000$ per fatal collision, $\$ 101,000$ per injury collision, and $\$ 10,000$ per PDO collision. A summary of the estimated social costs are found in Table 4.10 .

The monetary costs used in Chapter 6 will be based on these base-case costs (2016 CAD). It is important to note, however, that these estimated social collision costs have not been challenged in the economics literature. Therefore, presenting a sensitivity analysis in Chapter 6 will give an impression of the dynamics of the situation.

These social costs of collisions can shed a light on the effect of RLCs as to whether they are reducing the number of collisions, hence the costs to society. The 
Table 4.10 - Ontario's estimated social costs of traffic collisions

\begin{tabular}{|r|r|r|r|r|}
\hline \multirow{2}{*}{ Year } & \multirow{2}{*}{ Inflation Factor* } & \multicolumn{2}{|c|}{ Base-case values from (Vodden et al., 2007) } \\
\cline { 3 - 5 } & & \multicolumn{1}{c|}{ PDO } & \multicolumn{1}{c|}{ Injury } & \multicolumn{1}{c|}{ Fatal } \\
\hline \hline 2004 & $1.9 \%$ & $\$ 8,000$ & $\$ 82,000$ & $\$ 15,700,000$ \\
\hline 2005 & $2.2 \%$ & $\$ 8,172$ & $\$ 83,766$ & $\$ 16,038,109$ \\
\hline 2006 & $1.7 \%$ & $\$ 8,308$ & $\$ 85,154$ & $\$ 16,303,934$ \\
\hline 2007 & $1.9 \%$ & $\$ 8,465$ & $\$ 86,770$ & $\$ 16,613,222$ \\
\hline 2008 & $2.1 \%$ & $\$ 8,645$ & $\$ 88,611$ & $\$ 16,965,758$ \\
\hline 2009 & $0.5 \%$ & $\$ 8,691$ & $\$ 89,079$ & $\$ 17,055,287$ \\
\hline 2010 & $2.5 \%$ & $\$ 8,907$ & $\$ 91,294$ & $\$ 17,479,475$ \\
\hline 2011 & $2.9 \%$ & $\$ 9,166$ & $\$ 93,955$ & $\$ 17,988,868$ \\
\hline 2012 & $1.3 \%$ & $\$ 9,287$ & $\$ 95,190$ & $\$ 18,225,369$ \\
\hline 2013 & $1.0 \%$ & $\$ 9,377$ & $\$ 96,119$ & $\$ 18,403,323$ \\
\hline 2014 & $2.4 \%$ & $\$ 9,603$ & $\$ 98,426$ & $\$ 18,845,002$ \\
\hline 2015 & $1.2 \%$ & $\$ 9,718$ & $\$ 99,607$ & $\$ 19,071,142$ \\
\hline 2016 & $1.8 \%$ & $\$ \mathbf{8 9 3}$ & $\$ \mathbf{1 0 1 , 4 0 0}$ & $\mathbf{\$ 1 9 , 4 1 4 , 4 2 3}$ \\
\hline
\end{tabular}

*Factors obtained from Statistics Canada (2017)

observed number of collisions before and after the RLCs were installed, presented earlier in Table 4.3 of Sec. 4.2 , indicated a reduction in angle and turning movement collisions with an increase in rear-end and sideswipe collisions. Since the severities of the collision types differ, it was important to combine both frequency and severity related effects into one cost analysis to determine the overall observed effect of RLCs (i.e. angle and fatal, angle and injury, angle and PDO). Finally, the difference between the social costs of collisions observed before and after-period at the treated intersections were computed as an initial assessment of costs based on the trend.

Table 4.11 and Table 4.12 present the top three intersections that have benefited from the RLC treatment and the top three intersections that have been compromised based on the social costs of the average annual number of observed total collisions before and after the RLC treatment. Results indicate that the intersection that has benefited the most from the RLC treatment in Ottawa is King Edward Ave. and St. Patrick St., by a reduction in cost of an estimated $\$ 3.7$ million. 
Table 4.11 -Top 3 RLC intersections that benefited after the RLC installation

\begin{tabular}{|c|c|c|c|}
\hline \multicolumn{4}{|c|}{ Benefited } \\
\hline Intersection Name & $\begin{array}{l}\text { Average Annual } \\
\text { Total Cost } \\
\text { BEFORE RLC }\end{array}$ & $\begin{array}{c}\text { Average } \\
\text { Annual Total } \\
\text { Cost AFTER } \\
\text { RLC } \\
\end{array}$ & $\begin{array}{c}\text { Difference in } \\
\text { Cost }\end{array}$ \\
\hline KING EDWARD AVE \& ST. PATRICK ST & $\$ 3,951,520$ & $\$ 180,544$ & $-\$ 3,770,976$ \\
\hline CONROY RD \& LORRY GREENBERG DR & $\$ 3,632,645$ & $\$ 139,116$ & $-\$ 3,493,528$ \\
\hline BOOTH ST \& OTTAWA RIVER PKWY & $\$ 2,567,981$ & $\$ 414,011$ & $-\$ 2,153,970$ \\
\hline
\end{tabular}

Table 4.12 -Top 3 RLC intersections that hindered after the RLC installation

\begin{tabular}{|l|r|c|c|}
\hline \multicolumn{1}{|c|}{ Require re-location consideration } \\
\hline Intersection Name & $\begin{array}{c}\text { Average Annual } \\
\text { Total Cost } \\
\text { BEFORE RLC }\end{array}$ & $\begin{array}{c}\text { Average } \\
\text { Annual Total } \\
\text { Cost AFTER } \\
\text { RLC }\end{array}$ & $\begin{array}{c}\text { Difference in } \\
\text { Cost }\end{array}$ \\
\hline \hline CYRVILLE RD \& INNES RD & $\$ 449,671$ & $\mathbf{\$ 5 , 3 1 4 , 8 5 6}$ & $\$ 4,865,184$ \\
\hline HERON RD \& RIVERSIDE DR & $\$ 1,187,128$ & $\mathbf{\$ 4 , 2 7 1 , 5 9 0}$ & $\$ 3,084,463$ \\
\hline BELFAST RD \& ST. LAURENT BLVD & $\$ 663,914$ & $\mathbf{\$ 1 , 1 0 0 , 5 6 7}$ & $\$ 436,653$ \\
\hline
\end{tabular}

The intersection that is estimated to have had the least success in reducing the social costs after the RLC installation was the Cyrville Road and Innes Road intersection with an increase of $\$ 4.8$ million. The major increase in cost is due to a fatal collision in the post-treatment period. Relocation of RLCs from Table 4.12 need to be studied and considered when evaluation assessments prove that they no longer serve their original purpose.

It is important to note that one of the technical challenges in estimating the benefits and social costs of RLCs at intersections is the extremely rare occurrence of fatal collisions, which account for less than 1 percent of all the collisions but has an most impact on society. The comprehensive cost assigned to a fatality is around 30 times the injury collision costs within the same collision type; thus, one or two fatalities in either 
the pre or post-treatment period for a given intersection could greatly affect the costrelated analysis. While this could be legitimate if one could expect repeated similar sites to have the same number of fatalities, this is not likely, due to the rare and random occurrence of fatal collisions.

In a collision-cost research conducted for the State of New Mexico by Hall (1998), the author noted that the main factors determining whether an injury is a fatality rather than a severe injury is probably not affected by roadway-related treatments. They are more likely to be related to factors such as occupant age, restraint use, and type and size of vehicles involved. The author argues that such small numbers of fatalities should not be allowed to affect decisions on roadway-based treatments such as RLCs.

A total of eight fatal collisions took place in the 15 year period for the 34 RLC intersections in Ottawa. A visual representation of the geographical dispersion of the fatal collisions is presented in Figure 4.5. Given that the collision occurrence is random with significant variation over time and space, it is hard to judge whether a specific intersection is safe or safer than other intersections solely based on the RLC presence and frequency of fatal collisions in a given year. A further examination of some potential factors is performed in the next chapter. 


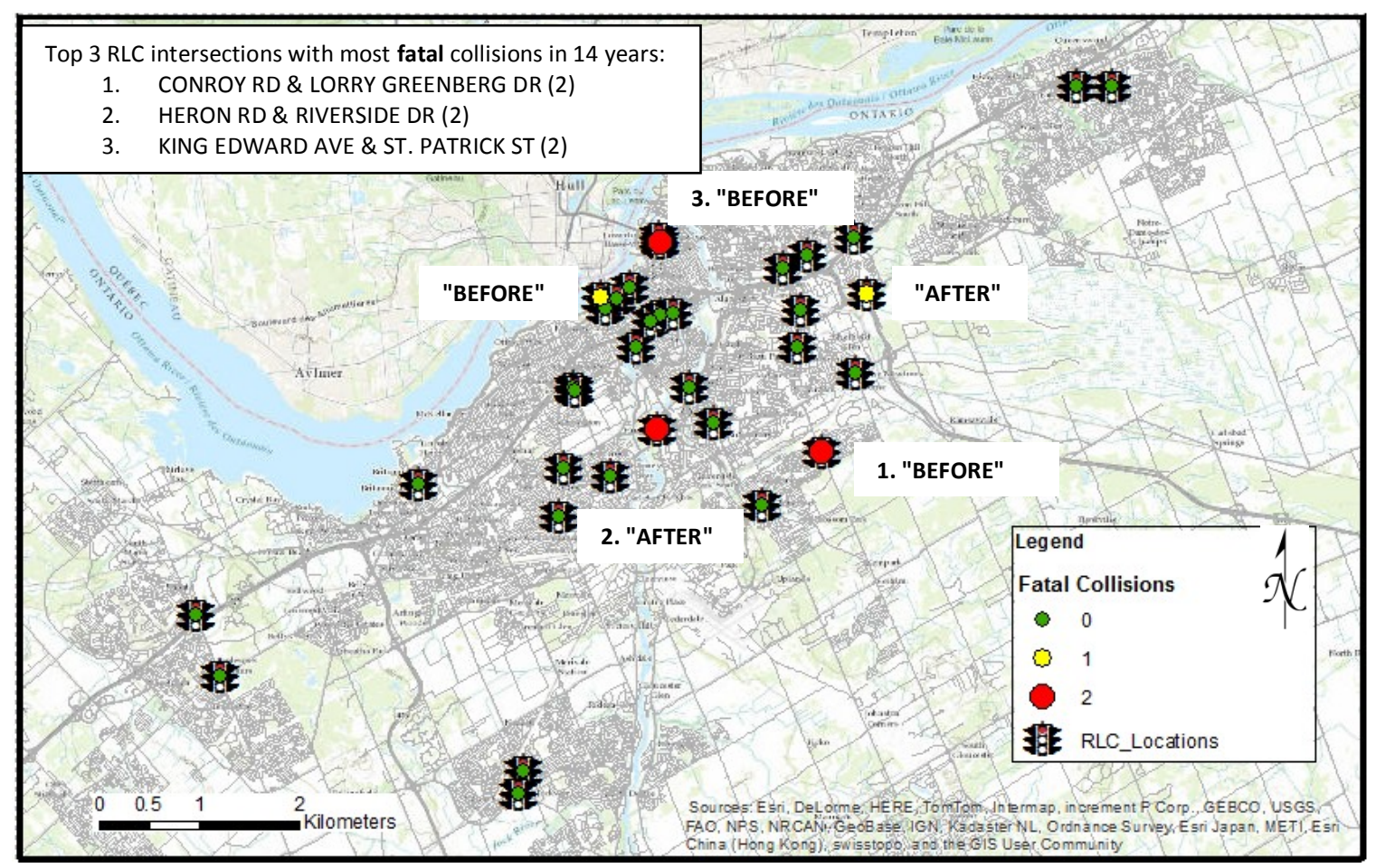

Figure 4.5 - RLC intersections with fatal collisions 


\section{Traffic Safety Evaluation: City of Ottawa Case Study}

This chapter presents the main results of the statistical analyses in examining the effect of RLCs on traffic safety using data from the City of Ottawa. Section 5.1 covers the crosssectional evaluation methods. Section 5.2 presents models with interaction terms followed by Section 5.3 presenting models with spillover effect evaluation. These sections are followed by Section 5.4 presenting the EB method analysis. Section 5.5 presents the case-control analysis results and finally, Section 5.6 presents a summary and comparison of the results.

\subsection{Cross-Sectional Evaluation}

This section aims to investigate whether some of the variation in the number of traffic collisions is explained in part by the presence of the RLC treatment (by controlling for multiple potential confounding factors). First, to understand the distribution of collisions, a histogram for each collision impact type was plotted confirming that the collisions count data is left-skewed, see Appendix C. This data profile indicates that ordinary least squares (OLS) regression would be inappropriate, since the distribution is non-linear and does not allow for negative values. Since collisions represent a form of count data, it is more appropriate to use a Poisson or a NB based estimation procedure.

The next step was to determine the proper regression model (either Poisson or NB) for estimating the relationship between the intersection attributes and collision experience. The mean and variance were estimated for all collision impact types and the

results are presented in Appendix D. The variance for all collision types is larger than the mean, displaying signs of over-dispersion. This confirms that the NB model is more 
appropriate than the Poisson model. Therefore, the Poisson models will not be tested any further.

The dependent variable used in the models is the number of collisions per year. Five count variables are used as dependent variables, which are the different collision impact types presented earlier in Table 4.6, which will be testing different outcomes. When it comes to interpreting the regression coefficients, the exponent of the coefficients is calculated as an odds ratio or incidence-rate ratio (IRR) (Buis, 2010). The IRR is the outcome rate while holding all other variables in the model constant. An IRR above (below) 1 implies that the independent variable is positively (negatively) related to the outcome.

An all-inclusive model, with all basic independent variables from Table 4.6, was run first on each collision impact type. The all-inclusive models give an insight to the overall significance and effect of the variables. However, these models were eventually reduced to a best fit model based on the statistical significance of the variables ( $p$-value $<0.05$ ). Model selection was based on comparisons through application of the goodnessof-fit statistical tests using likelihood ratios (LRs) and AIC. All models were checked for multi-collinearity issues (i.e., independent variables that are correlated) and the results did not indicate any correlation issues between the variables. Table 5.1 illustrates a summary of the all-inclusive models and the selected best fit models. The full StataSE output of all models including all their goodness-of-fit parameters is found in Appendix E.

The results indicate that the RLC effect on the total number of collisions is an estimated slight increase of less than $1 \%(\operatorname{IRR}=1.0094)$. However, the $p$-value for the 
Table 5.1 - NB regression results based on best fit models (IRR coefficients)

\begin{tabular}{|c|c|c|c|c|c|c|c|c|c|c|}
\hline \multirow{2}{*}{ Variables } & \multicolumn{2}{|c|}{ Total } & \multicolumn{2}{|c|}{ Rear-end } & \multicolumn{2}{|c|}{ Angle } & \multicolumn{2}{|c|}{ Turning movement } & \multicolumn{2}{|c|}{ Sideswipe } \\
\hline & All-inclusive & Best Fit & All-inclusive & Best Fit & All-inclusive & Best Fit & All-inclusive & Best Fit & All-inclusive & Best Fit \\
\hline rlcinstalled 2 & 1.0200 & 1.0094 & $1.1744 * *$ & $1.1791^{* *}$ & $0.8240 * *$ & $0.8317^{*}$ & $0.8319 *$ & $0.8365 *$ & 1.1910 & 1.1854 \\
\hline lnaadt & $1.8523 * * *$ & $1.8568 * * *$ & $3.2585^{* * *}$ & $3.2921 * * *$ & 0.8661 & 0.8643 & $1.5975 * * *$ & $1.5879 * * *$ & $2.7179 * * *$ & $2.6136^{* * *}$ \\
\hline median & $0.6665^{* * *}$ & $0.6798 * * *$ & 0.9893 & & $0.7379 * *$ & $0.7592 * *$ & $0.4364 * * *$ & $0.4268^{* * * *}$ & $0.5594 * * *$ & $0.5719^{* * * *}$ \\
\hline channelization & $1.4449 * * *$ & $1.4369^{* * *}$ & $1.4925 * * *$ & $1.4821 * * *$ & 1.0853 & & $1.7747 * * *$ & $1.7417 * * *$ & $1.4696^{* *}$ & $1.4461 * *$ \\
\hline leftturn & $1.1385^{* * *}$ & $1.1404 * * *$ & 1.0386 & & $1.2619^{* * *}$ & $1.2644 * * *$ & 0.9233 & & $1.4711^{* * *}$ & $1.4681^{* * *}$ \\
\hline plt & $1.1760 * * *$ & $1.1389 * *$ & 1.0506 & & 1.0131 & & $1.2614 *$ & $1.3181 * *$ & $1.9306^{* * *}$ & $1.8557 * * *$ \\
\hline fplt & 1.0764 & & $1.3124 * * *$ & $1.2805^{* * *}$ & $0.7143 * * *$ & $0.7288 * * *$ & 0.9389 & & $1.6150 * * *$ & $1.4686^{* * *}$ \\
\hline pplt & 1.0787 & & $1.3143 * * *$ & $1.3015^{* * *}$ & $0.4935 * * *$ & $0.5019^{* * *}$ & $1.9326^{* * *}$ & $1.9257 * * *$ & 1.1043 & \\
\hline speed1 & 1.0064 & $1.0073 * * *$ & $1.0150^{* * *}$ & $1.0147 * * *$ & 1.0017 & & 0.9934 & & 0.9968 & \\
\hline speed 2 & $1.0098^{* * *}$ & $1.0101 * * *$ & $1.0176^{* * *}$ & $1.0183 * * *$ & 0.9979 & & 1.0056 & & 0.9889 & \\
\hline thrulanes & $1.1898 * * *$ & $1.1799 * * *$ & 1.0326 & & $1.3399 * * *$ & $1.3279^{* * *}$ & 1.1169 & $1.1448^{*}$ & $1.5524 * * *$ & $1.5016^{* * *}$ \\
\hline cons & $0.0058^{* * *}$ & $0.0057 * * *$ & $<0.001 * * *$ & $<0.001^{* * *}$ & $9.9773 * * *$ & $10.2365^{* *}$ & $0.0189 * * *$ & $0.0168 * * *$ & $<0.001 * * *$ & $<0.001 * * *$ \\
\hline \multicolumn{11}{|c|}{ Goodness-of-fit parameters } \\
\hline AIC & 4411 & 4410 & 3516 & 3510 & 2971 & 2964 & 2902 & 2899 & 2220 & 2218 \\
\hline $\begin{array}{r}\text { Maximum } \\
\text { Likelihood R }^{2} \\
\end{array}$ & 0.393 & 0.390 & 0.501 & 0.500 & 0.181 & 0.179 & 0.185 & 0.179 & 0.212 & 0.209 \\
\hline Observations & 685 & 685 & 685 & 685 & 685 & 685 & 685 & 685 & 685 & 685 \\
\hline $\begin{array}{l}\text { RLC } \\
\text { EFFECT*: }\end{array}$ & $2.0 \%$ & $0.9 \%$ & $17.4 \%$ & $17.9 \%$ & $-17.6 \%$ & $-16.8 \%$ & $-16.8 \%$ & $-16.3 \%$ & $19.1 \%$ & $18.5 \%$ \\
\hline
\end{tabular}

Exponentiated coefficients $=* \mathrm{p}<0.05 * * \mathrm{p}<0.01 * * * \mathrm{p}<0.001$

* A negative sign indicates a decrease in collisions. 
RLC treatment variable (rlcinstalled2) was 0.801 which is far above the generally accepted maximum threshold of 0.05 that is needed to reject the null hypothesis of no significant effect. This result suggests that the observed difference of the RLC treatment in the total collisions is plausibly a chance finding and that the hypothesis that there is no difference between the treatment and non-treatment intersections cannot be rejected. Failing to reject the null hypothesis according to the standard criteria of statistical significance, however, does not mean definitively that there is no treatment effect (Altman \& Bland, 1995). It is important to note however, that it is the size of the effect that determines the importance, not the presence of statistical significance (Crombie \& Davis, 2009).

The model coefficients for angle and turning movement collisions show that the RLC has a significant effect in reducing the number collisions by $17 \%(\mathrm{IRR}=0.8317)$ and $16 \%(I R R=0.8365)$, respectively. On the other hand, the presence of a RLC increases the rear-end collisions by $18 \% \quad(I R R=1.1791)$ and sideswipe collisions by $19 \%$ (IRR=1.1854). The $p$-values were also calculated to assess whether the results are likely to have occurred simply through chance. The $p$-value was $<0.05$ for the rear-end, angle and the turning movement collision models, suggesting a strong degree of statistical significance; thus the findings are highly unlikely to have arisen by chance. Therefore, the null hypothesis that there is no difference between the treated and non-treatment is strongly rejected. The $p$-value for sideswipe collisions was insignificant $(>0.05)$, suggesting that the observed difference of the RLC treatment in sideswipe collisions is plausibly a chance finding. 
To complement the NB models, RENB and FENB regression models were also tested. The models were run on the panel data to model the collisions between intersections as well as over the time (measuring both cross-sectional and time-series effect). Several non-linear panel models were estimated using the xtnbreg command in StataSE, starting with a saturated model that involves all the possible explanatory variables considered in this study. A procedure of eliminating insignificant variables using manual backward method was undertaken and the most parsimonious model was obtained on the basis of minimizing the AIC value.

Table 5.2 presents a summary of the coefficient estimates of the RLC independent variable from the FENB and RENB final models. Not all the estimated coefficients were reported in the table to save space and focus on the RLC treatment effect. Detailed output of the full set of results can be found in Appendix F.

Table 5.2 - FE and RE NB regression results for RLC effect

\begin{tabular}{|c|c|c|c|c|}
\hline \multirow{2}{*}{$\begin{array}{c}\text { Collision } \\
\text { Impact Type }\end{array}$} & \multicolumn{4}{|c|}{ RLC (rlcinstalled2) variable IRR coefficient } \\
\hline & $\begin{array}{c}\text { Fixed Effect } \\
\text { NB } \\
\end{array}$ & $\begin{array}{c}\text { Random Effect } \\
\text { NB } \\
\end{array}$ & $\begin{array}{c}\text { Hausman Test: } \\
\text { Prob>chi2 }\end{array}$ & Preferred test \\
\hline Total & $-0.01 \%$ & $0.01 \%$ & $<0.0001$ & Fixed effect \\
\hline Rear-end & $23.4 \%{ }^{* * *}$ & $23.8 \% \%^{* * *}$ & 0.8780 & Random Effect \\
\hline Angle & $\begin{array}{r}\text { model doesn't } \\
\text { converge }\end{array}$ & $-28.5 \%{ }^{* * * *}$ & N/A & Random Effect \\
\hline $\begin{array}{l}\text { Turning } \\
\text { movement }\end{array}$ & $-13.5 \%$ & $-14.0 \%$ & 0.2365 & Random Effect \\
\hline Sideswipe & $38.9 \%{ }^{* * *}$ & $34.5 \%$ ** & N/A & Random Effect \\
\hline
\end{tabular}

Exponentiated coefficients $=* \mathrm{p}<0.05 * * \mathrm{p}<0.01 * * * \mathrm{p}<0.001$

The results from the FE and RE models were compared using Hausman's test, which examines whether a significant correlation exists between RE (unique errors $u_{\mathrm{i}}$ ) and explanatory variables, the null hypothesis is they are not. In the presence of such correlation, the RE slope estimator is inconsistent; consequently, the FE model should be 
chosen over the RE model. If the correlation is insignificant ( $p$-value $>0.05$ ) then it is safe to use RE models (Princeton University Library, 2017; Torres-Reyna, 2007).

The resulting FENB and RENB models for all collision impact types indicate that the models outperform the NB model from the AIC values. The statistically significant $p$ value of the Hausman for the total collisions model indicates there may be a systematic difference in the coefficients, and the FE model is preferred over the RE model. For the remaining models the RE models were determined to be better fit.

The results of the effect of the RLC on all the collision impact types are in line with the estimates based on the NB regression. They show that after accounting for observable characteristics, the average treatment effect on total collisions is a very slight decrease of under $1 \%$, an increase in rear-end and sideswipe collisions of $24 \%$ and $35 \%$, and a decrease in angle and turning movement collisions of $29 \%$ and $14 \%$, respectively.

To determine whether the ZINB models provide an improvement over the traditional NB model, ZINB regression was carried out in StataSE. The comparison between the NB and the ZINB models were evaluated using the Vuong test to identify the best fit model. Detailed output of the comparisons between the NB and ZINB models for each collision impact type are available in Appendix G. For all dependent variables, Vuong's test suggested that the standard NB models perform better than the ZINB models for all the collision impact types reviewed. This result suggests that the role of RLCs is much more important to how often collisions occur, but not to whether they occur or not.

Based on the outlined findings, the RENB panel model approach seems to be the most appropriate for the data in this thesis according to the modeling fitness results which 
successfully modelled the RLC effect on collision occurrence with correction spatial and temporal changes. Ignoring spatial correlation in collision modeling may lead to biased parameter estimation by not capturing the multivariate nature of the collision data. This modeling technique can be used to model any non-negative integer count data in a form of panel structure in future research.

\subsection{Interaction Terms Evaluation}

To try to achieve better balance in the models, richer models were specified with interactions between the RLC treatment and the other covariates. Section 5.1 reported the main effects of variables using the FE and RE models and this section includes the main effects plus the interaction terms between the RLC variable and all the covariates in a separate model.

The FE model did not converge for total collisions when interaction term were added. This means the model has too many factors and not a big enough sample size, and cannot be fit, therefore, a RE model was used instead. RE regression models were first run including all the main effects and interaction terms. Next, manual backward selection was performed to eliminate non-significant pair-wise interactions, while forcing the main effects into the model at this stage for interpretation purposes. Once non-significant pairwise interactions have been eliminated, backwards selection is considered again to eliminate any non-significant main effects that are not involved in remaining interaction terms (Williams, 2016). Details from the StataSE output of the models for all collisions impact types are found in Appendix $\mathrm{H}$.

In order to calculate the predicted value of the dependent variable for each observation, margins were used in StataSE. The way it works is that it calculates the 
outcome if all intersections would have a RLC installed (coded as 1), keeping the other variables as they happen to be, and then if all intersections would not have a RLC installed (coded as 0), keeping the other variables as they happen to be. So for each value (i.e. 0 and 1) the margins command calculates what the mean predicted value of the dependent variable would be if all observations had that value for the binary variable. To find out whether this difference is significant, the marginal effects are computed.

To examine the marginal effects, which is the derivative of the mean predicted value with respect to the RLC covariate, the dydx option was used in StataSE with the margins command and the results are shown in Table 5.3. The effect of a RLC installed vs. no RLC on total collisions is an average decrease of 0.0467 collisions. This is simply the difference in the predictive margins. The difference is not significant with a $p$-value of 0.2670 .

Table 5.3 - Marginal effect of the RLC covariate (xtnbreg* estimates)

\begin{tabular}{|l|r|r|r|r|r|r|}
\hline Collision impact type & $\begin{array}{l}\text { dy/dx } \\
\text { Coef. }\end{array}$ & \multicolumn{1}{c|}{ Std. Err. } & \multicolumn{1}{c|}{$\mathbf{z}$} & \multicolumn{2}{c|}{ P>chi2 } & \multicolumn{2}{|c|}{ [95\% Conf. Interval] } \\
\hline \hline Total collisions & -0.0467 & 0.0420 & -1.11 & 0.2670 & -0.1291 & 0.0357 \\
\hline Rear-end & 0.1364 & 0.0573 & 2.38 & 0.0170 & 0.0241 & 0.2486 \\
\hline Angle & -0.5334 & 0.0904 & -5.90 & $<0.0001$ & -0.7107 & -0.3561 \\
\hline Turning movement & -0.5946 & 0.1304 & -4.56 & $<0.0001$ & -0.8501 & -0.3390 \\
\hline Sideswipe & 0.2451 & 0.0934 & 2.62 & 0.0090 & 0.0620 & 0.4282 \\
\hline
\end{tabular}

*refers to the StataSE command for FE and RE models

The dy/dx coefficient from Table 5.3 was combined with the average annual observed collisions per intersection per year (from Table 4.3) for the post-treatment period to calculate the percent change has the RLC not been installed. Results are shown in Table 5.4.

Similar to the results obtained from the NB analysis in Section 5.1, the RLC seem to have a significant effect in reducing the number of angle and turning movement 
Table 5.4 - Impact of RLC in terms of percentage of collision reduction (xtnbreg)

\begin{tabular}{|c|c|c|c|c|c|}
\hline $\begin{array}{l}\text { Collision Types \& } \\
\text { Classification }\end{array}$ & $\begin{array}{c}\text { AAOC }^{\mathrm{a}} \\
\text { (Before RLC) }\end{array}$ & $\begin{array}{c}\mathrm{AAOC}^{\mathrm{a}} \\
\text { (After RLC) }\end{array}$ & $\begin{array}{l}\text { dy/dx } \\
\text { Coef. }\end{array}$ & $\begin{array}{c}\text { Effect without } \\
\text { RLC } \\
(2)-(3)\end{array}$ & $\begin{array}{c}\text { Percent } \\
\text { Change } \\
(2)-(4) /(4)\end{array}$ \\
\hline & (1) & (2) & (3) & (4) & (5) \\
\hline Total Collisions & 14.18 & 16.72 & -0.0467 & 16.77 & $-0.3 \%$ \\
\hline Rear-end & 5.00 & 7.88 & 0.1364 & 7.74 & $1.8 \%$ \\
\hline Angle & 3.82 & 3.08 & -0.5334 & 3.61 & $-14.8 \%$ \\
\hline Turning movement & 3.03 & 2.69 & -0.5946 & 3.28 & $-18.1 \%$ \\
\hline Sideswipe & 1.35 & 1.96 & 0.2451 & 1.71 & $14.3 \%$ \\
\hline
\end{tabular}

${ }^{\mathrm{a}} \mathrm{AAOC}=$ Average annual observed collisions per intersection.

${ }^{\mathrm{b}}$ Contrast coefficients obtained from Table 5.3.

collisions by $15 \%$ and $18 \%$, respectively. By contrast, the number of rear-end and sideswipe collisions increase by $2 \%$ and $14 \%$, respectively. The estimation results of the individual interaction terms between each road elements, the RLC, and collision frequencies for each collision impact type uncovered important interactions. The significance of an interaction can be determined if the confidence interval includes 0 . If it does, the predictor is not significant (Hilbe, 2008). Detailed output results of the regression models with the interaction terms are available in Appendix $\mathrm{H}$.

Another way to evaluate the significance of the interaction is looking at the graphical plots of the marginal effects. The structure of the significant interactions was summarized graphically (using the marginsplot command in StataSE ) by plotting the logits and relative risks from the regression coefficients for main effect and interaction parameters. When the difference in response between RLC and non-RLC intersections was constant throughout the range (slope of lines are parallel and do not cross) this indicates that there is no interaction between the two variables. When RLC has a steeper/or a less steep covariate-curve compared to non-RLC intersections, this indicates that it has an effect with an interaction, given enough statistical power (Joseph, 2016). 
Figure 5.1 presents the significant interaction terms between the RLC and the different covariates for the total collisions. Figure 5.2 presents the non-significant interaction terms for the total collisions to show the difference in the lines of the plots. As an example of interpreting the results, looking at graph "a" in Figure 5.1, it can be observed that while non-RLC intersections start out with higher frequency of total collisions when no right-turn channelization is present, RLC intersections surpass the number of total collisions of the non-RLC intersections when there is right-turn channelization.

Results for total collisions indicate that the interaction terms of RLC with rightturn channelization (rlcinstalled $x$ channelization), RLC with number of left-turns lanes (rlcinstalled $x$ leftturn), RLC with the number of thru lanes (rlcinstalled $x$ thrulanes), RLC with median (rlcinstalled $x$ median), and RLC with permitted left-turn signals (rlcinstalled $x$ plt) turned out to be significant.

On the other hand, it was found that when testing the interaction terms of RLC with speed limit of Road 1 (rlcinstalled $x$ speed1), RLC with protected-permitted left-turn signals (rlcinstalled $x$ pplt), RLC with fully-protected left-turn signals (rlcinstalled $x$ fplt) the difference in response between RLC and non-RLC intersections was constant throughout the range, as seen in Figure 5.2 in graphs f) to h). The slope of lines are parallel and do not cross, confirming that there is no interaction was apparent. Therefore, it could be concluded that these interaction terms are not necessary to be included in the model to help explain the effects of RLC, and did not contribute to explaining the variation in the collision outcomes. Therefore, they were dropped from the final model. 


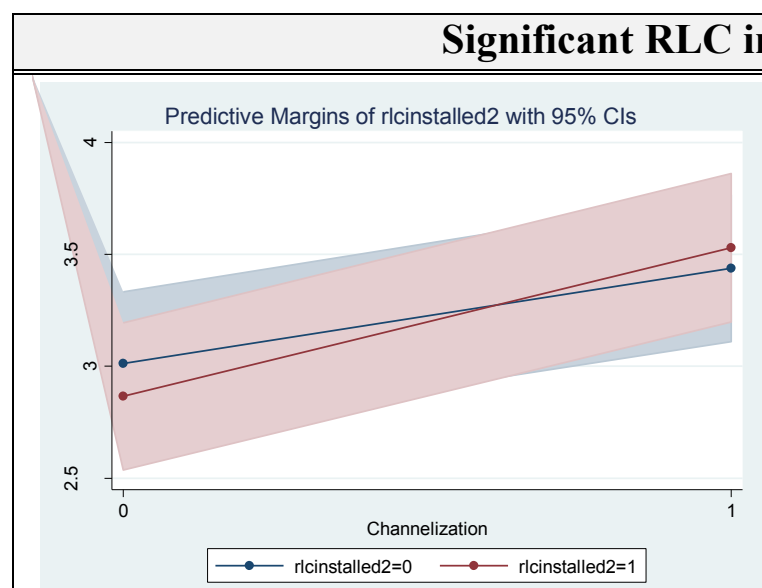

a) RLC presence $x$ Presence of right-turn channelization

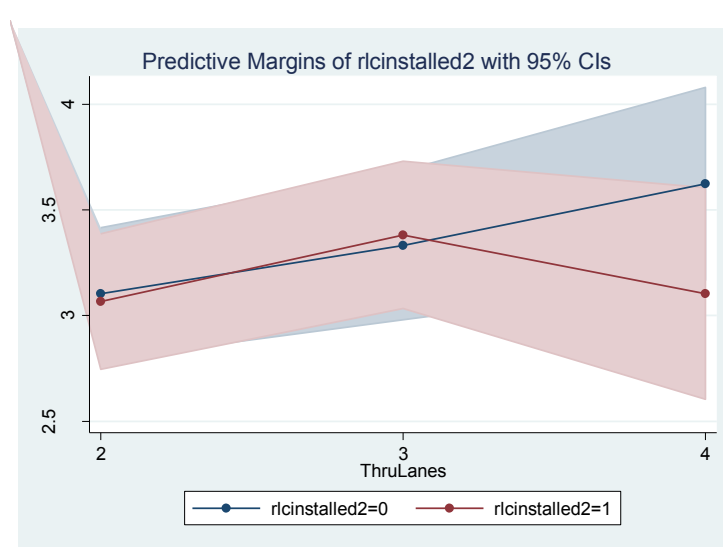

c) RLC presence $x$ number of thru lanes

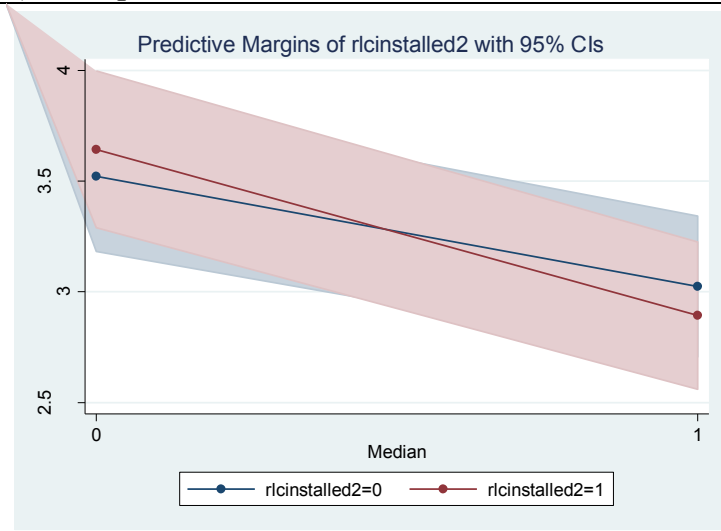

e) RLC presence $x$ presence of median
Predictive Margins of rlcinstalled 2 with $95 \% \mathrm{Cls}$

b) RLC presence $x$ Number of left-turn lanes

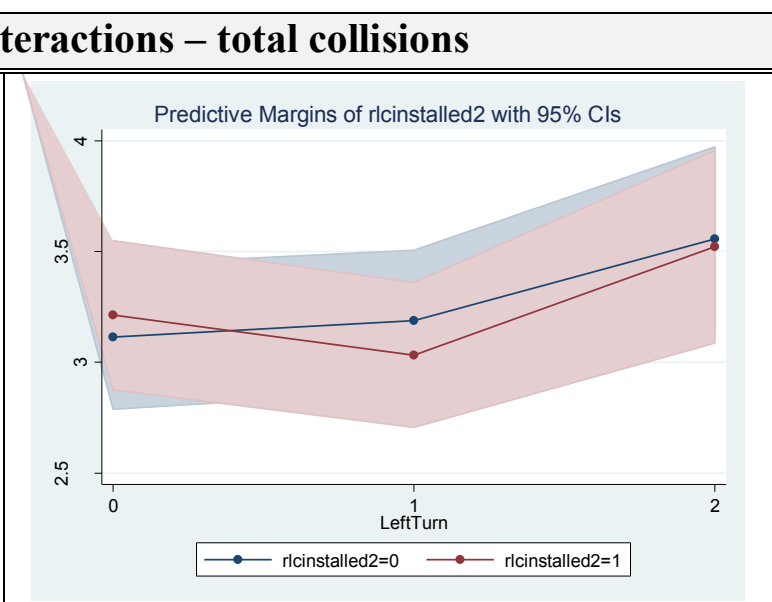

d) RLC presence $x$ permitted left-turn signal

Figure 5.1 - Significant interaction terms for total collisions 


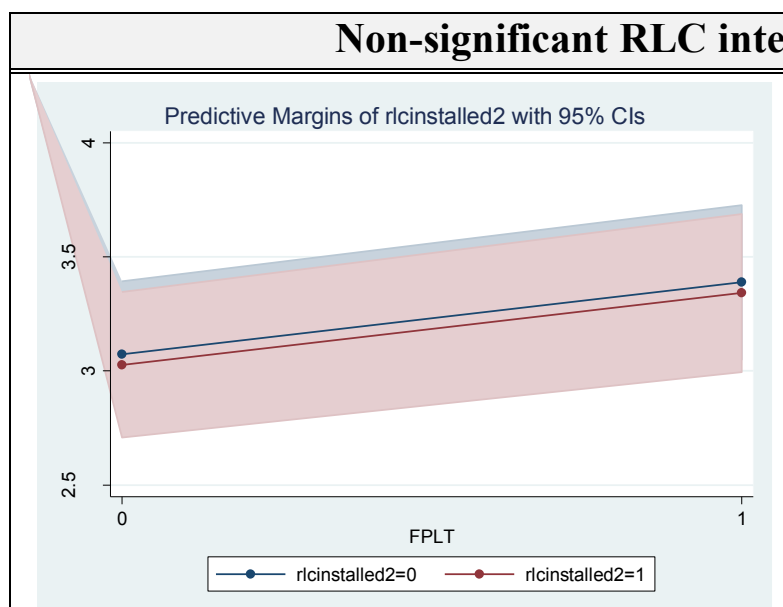

f) RLC presence $x$ fully-protected left-turn signal

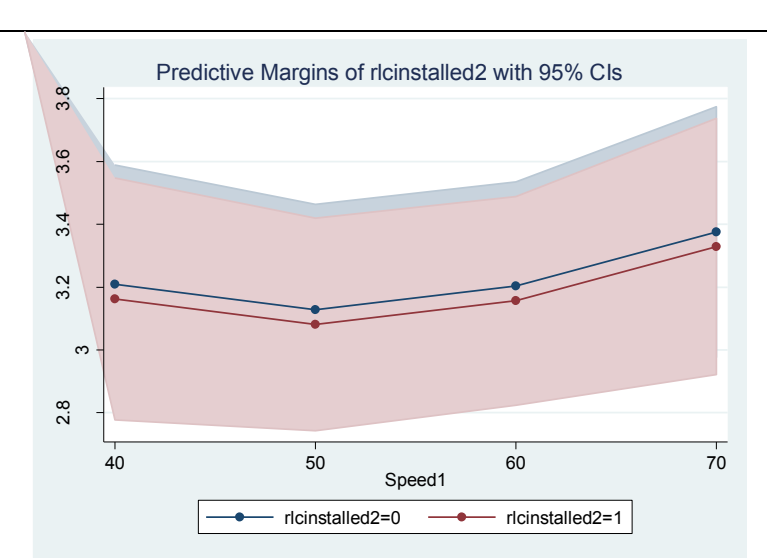

h) RLC presence $x$ speed on road 1

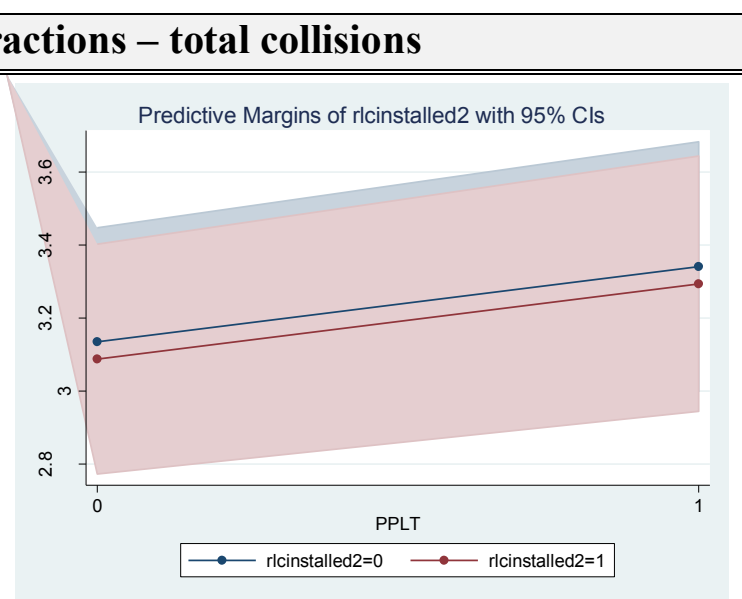

g) RLC presence x protected-permitted left-turn signal

\section{Figure 5.2 - Non-significant interaction terms for total collisions}

One reason for empirically assessing interactions is that it can provide insight into the mechanisms for the outcome. It is possible to detect intersections for whom an outcome would occur if both exposures are present but would not occur if just one or the other were present.

The estimation results from the models show that site-varying factors coupled with the RLC may significantly influence the collision frequencies by impact type. A summary of the significant interactions between the RLC and the different covariates for the remaining collision impact types are presented in Table 5.5. The percentages of 
Table 5.5 - Significant interactions of RLC with different covariates according to collision type and classification (xtnbreg IRR coefficients)

\begin{tabular}{|c|c|c|c|c|c|}
\hline \multirow{2}{*}{ Variables } & Total & Rear-end & Angle & TM & Sideswipe \\
\hline & \multicolumn{5}{|c|}{ Determining effects of significant interactions with the RLC } \\
\hline rlcinstalled 2 & $V_{<1 \%}$ & 个2\% & $V_{15 \%}$ & $v_{18 \%}$ & $\overline{1} 14 \%$ \\
\hline median & $\sqrt{22 \%}$ & & $\sqrt{52 \%}$ & $\sqrt{45 \%}$ & $\sqrt{41 \%}$ \\
\hline channelization & 个 $27 \%$ & 个 $39 \%$ & & & \\
\hline lefturn & $\begin{array}{l}\text {-With } 1 \text { left-turn } \\
\text { lane } V_{22 \%} \\
\bullet \text { With } 2 \text { left-turn } \\
\text { lanes } V_{13 \%} \\
\text { (non-significant) }\end{array}$ & & & $\begin{array}{c}\text { - With } 1 \text { left-turn } \\
\text { lane } \mathbf{6 6 \%} \\
(\text { non-significant }) \\
\text { - With } 2 \text { left-turn } \\
\text { lanes } \uparrow 290 \%\end{array}$ & \\
\hline plt & $V_{49 \%}$ & & $V_{52 \%}$ & $V_{75 \%}$ & $\sqrt{43 \%}$ \\
\hline fplt & & & & $V_{75 \%}$ & \\
\hline pplt & & & $\sqrt{51 \%}$ & $V_{60 \%}$ & \\
\hline \multicolumn{6}{|l|}{ speed1 } \\
\hline \multicolumn{6}{|l|}{ speed 2} \\
\hline thrulanes & $\begin{array}{c}\text { - With } 2 \text { thru lanes } \\
<<1 \% \\
\text { (non-significant) } \\
\text { - With } 3 \text { thru lanes } \\
8 \% \\
\text { - (non-significant }) \\
\text { - With } 4 \text { thru lanes } \\
38 \%\end{array}$ & & & $\begin{array}{c}\text { - With } 2 \text { thru lanes } \\
20 \% \\
\text { (non-significant) } \\
\text { - With } 3 \text { thru lanes } \\
\text { - With } 4 \text { thru lanes } \\
\text { (non-significant) }\end{array}$ & \\
\hline
\end{tabular}

increase and decrease are obtained from the IRR values of the interaction effects from the best fit models, which can be found in Appendix H.

As an example of interpreting the results, when looking at total collisions, an intersection with the presence of a median coupled with a RLC would decrease the chance of collisions by $22 \%(I R R=0.7760)$. On the other hand, the presence of right-turn channelization coupled with a RLC would increase the chance of total collisions by $27 \%$ $(\mathrm{IRR}=1.2685)$. Having one designated left-turn lanes would have a significant decrease the number of total collisions by $22 \%$ and having two designated left-turn lanes would also decrease the number of collisions by $13 \%$, which was not significant. An intersection 
with four thru lanes coupled with a RLC would significantly decrease the total number of collisions by $38 \%$. Having two thru lanes would decrease the number of collisions by less than $1 \%$ while having three lanes would increase the number of collisions by $8 \%$, respectively. However, these two values were not statistically significant. Among all the significant variables, the presence of a median is found to be the most influential siterelated factor contributing to the reduction of collision frequencies at intersections when coupled with a RLC.

In summary, one of the motivations for examining interactions is to identify which subgroups would benefit most from the RLC treatment when new sites are planned or when resources are limited. In most settings, it is not be possible to treat all intersections. Therefore, it is important to identify the subgroups which the RLC treatment is likely to have the largest effect on and would benefit the most. Even in settings in which resources are not limited and it is possible to treat several intersections at once, it may be the case that the RLC treatment is beneficial for some intersections and harmful for others. In such cases, it is very important to identify those groups for which treatment may be harmful and refrain from treating such intersections.

\subsection{Spillover Effect Evaluation}

Traffic flow outside of the immediate treatment zone and on alternate routes must be monitored to determine possible spillover effects. It is possible that drivers may choose alternative routes to avoid RLC sites, therefore, the collision reduction at RLC sites may include the effect induced by a reduced traffic flow. The benefits of RLCs will therefore be overestimated without controlling for the change in traffic flow. A NB regression model was run to check if there has been a redistribution of traffic flow caused by the 
RLC installations so that vehicle volume decrease in treated sites and increase in the surrounding area. Results indicated that change in traffic patterns was not significant.

Next, the time and distance halo variables were incorporated in the NB and RENB models with the interaction terms to assess whether the presence and number of the RLCs at a range of distances (from $0 \mathrm{~m}$ to $500 \mathrm{~m}$ and $500 \mathrm{~m}$ to $1 \mathrm{~km}$ ) from an intersection affects the frequency of collisions at the control intersections. The complete output of the models can be found in Appendix I.

The direction, statistical significance and magnitude of the RLC coefficients in the NB regression best fit models were unaffected from previous findings by the introduction of variables to control for time and distance-halos. Table 5.6 presents the marginal effect of the RLC covariate. Next, the marginal effect was used in Table 5.7 to estimate the impact of the RLC presence on the number of collisions.

Table 5.6 - Marginal effect of the RLC covariate (nbreg* estimates)

\begin{tabular}{|l|r|r|r|r|r|r|}
\hline \multicolumn{1}{|c|}{$\begin{array}{c}\text { Collision impact } \\
\text { type }\end{array}$} & \multicolumn{1}{c}{$\begin{array}{c}\text { dy/dx } \\
\text { Coef. }\end{array}$} & Std. Err. & \multicolumn{1}{c|}{$\mathbf{z}$} & P>chi2 & \multicolumn{1}{l|}{ [95\% Conf. Interval] } \\
\hline \hline Total collisions & 0.8846 & 0.6495 & 1.36 & 0.1730 & -0.3883 & 2.1575 \\
\hline Rear end & 0.6358 & 0.4529 & 1.40 & 0.1600 & -0.2518 & 1.5234 \\
\hline Angle & -0.4067 & 0.2715 & -1.50 & 0.1340 & -0.9387 & 0.1254 \\
\hline Turning movement & -0.2567 & 0.2600 & -0.99 & 0.3240 & -0.7663 & 0.2530 \\
\hline Sideswipe & 0.2616 & 0.3054 & 0.86 & 0.3920 & -0.3369 & 0.8601 \\
\hline
\end{tabular}

*refers to the StataSE command for NB regression models

The model estimations indicate a reduction in collisions resulting from the presence of the RLC for angle collisions by $12 \%$, and turning movement collisions by 9\%. Rear-end collisions are estimated to increase by $9 \%$, and sideswipe collisions increase by $15 \%$. Based on these estimations the effect of the RLC on total collisions is estimated at an increase of $6 \%$. The coefficient for the RLC effect was not significant in the models. 
Table 5.7 - Impact of RLC in terms of percentage of collision reduction (nbreg)

\begin{tabular}{|c|c|c|c|c|c|}
\hline $\begin{array}{c}\text { Collision Impact } \\
\text { Type }\end{array}$ & $\begin{array}{l}\text { AAOC }^{\text {a }} \\
\text { (Before } \\
\text { RLC) }\end{array}$ & $\begin{array}{c}\text { AAOC }^{\text {a }} \\
\text { (After } \\
\text { RLC) }\end{array}$ & $\begin{array}{l}\text { dy/dx } \\
\text { Coef. }\end{array}$ & $\begin{array}{l}\text { Effect without } \\
\text { RLC } \\
\text { (2) -(3) }\end{array}$ & $\begin{array}{l}\text { Percent } \\
\text { Change }^{b} \\
(2)-(4) /(4)\end{array}$ \\
\hline & (1) & (2) & (3) & (4) & (5) \\
\hline Total Collisions & 14.18 & 16.72 & 0.8846 & 15.84 & $5.6 \%$ \\
\hline Rear end & 5.00 & 7.88 & 0.6358 & 7.24 & $8.8 \%$ \\
\hline Angle & 3.82 & 3.08 & -0.4067 & 3.49 & $-11.7 \%$ \\
\hline Turning movement & 3.03 & 2.69 & -0.2567 & 2.95 & $-8.7 \%$ \\
\hline Sideswipe & 1.35 & 1.96 & 0.2616 & 1.70 & $15.4 \%$ \\
\hline
\end{tabular}

${ }^{\mathrm{a}} \mathrm{AAOC}=$ Average annual observed collisions per intersection.

${ }^{\mathrm{b}}$ Contrast coefficients obtained from Table 5.6.

The RENB best fit models, which can be found in Appendix I (Table A and Table B), lacked significance for the RLC coefficient for the total collisions model, the rear-end model and the turning movement model. The magnitude of the RLC effect on all models seemed to be underestimated based on the previous model estimations. Also, the direction of the RLC coefficient was the opposite of previous findings for the sideswipe collision impact type.

The results of the best fit model estimations are presented in Table 5.8. The RENB regression lumps the data of all the years of each intersection together when estimating a difference between the RLC and non-RLC intersections. Since there is a low number of comparable non-RLC intersections in this dataset, inferences might not be accurate using this method. Therefore, the NB regression will be used to better assess the spillover effects.

Examination of possible spillover effects of the RLC program demonstrated a moderate, yet significant, effect on collisions. Control intersections within $500 \mathrm{~m}$ of RLC 
Table 5.8 - Best fit regression models with time and distance halo variables (IRR coefficients)

\begin{tabular}{|c|c|c|c|c|c|c|c|c|c|c|c|}
\hline \multirow{2}{*}{\multicolumn{2}{|c|}{ Variables }} & \multicolumn{2}{|c|}{ Total } & \multicolumn{2}{|c|}{ Rear-end } & \multicolumn{2}{|c|}{ Angle } & \multicolumn{2}{|c|}{ Turning movement } & \multicolumn{2}{|c|}{ Sideswipe } \\
\hline & & nbreg & xtnbreg, re & nbreg & xtnbreg, re & nbreg & xtnbreg, re & nbreg & xtnbreg, re & nbreg & xtnbreg, re \\
\hline rlcinstalled2 & & 1.3217 & 1.3946 & 0.8926 & 1.1853 & $3.5431 * *$ & $2.0091 * *$ & $4.8251 * * *$ & 1.1457 & 1.4105 & $1.7296^{*}$ \\
\hline lnaadt & & $1.7490 * * *$ & $1.5541 * * *$ & $3.0504 * * *$ & $2.0208 * * *$ & 0.9445 & 1.0696 & $1.5684 * * *$ & $1.3274^{*}$ & $1.8796^{* * *}$ & $1.7035 * *$ \\
\hline median & & $0.70654 * * *$ & $0.62274 * * *$ & $1.7350 * * *$ & $1.8668 * *$ & $0.6991 * * *$ & 0.6854 & $0.3222 * * *$ & $0.56255^{* *}$ & $0.5310^{* * *}$ & $0.5716^{*}$ \\
\hline channelization & & $1.3639 * * *$ & $1.5410^{* * *}$ & 1.0304 & 1.2650 & $1.3925 * * *$ & 1.4162 & $1.9992 * * *$ & & $1.6995 * * *$ & $1.8077^{*}$ \\
\hline \multirow[t]{3}{*}{ thrulanes } & 2 & 0.9896 & 0.9665 & $0.4656^{* * *}$ & $0.4609 * *$ & $1.8955^{* * *}$ & 1.6186 & 1.2160 & & $1.6501 *$ & 1.5230 \\
\hline & 3 & 1.2091 & 1.2899 & $0.4004 * * *$ & $0.4176^{* *}$ & $2.7636^{* * * *}$ & $2.6110^{* *}$ & 1.1655 & & $4.2988 * * *$ & $2.5098^{*}$ \\
\hline & 4 & $2.4615^{* * *}$ & $1.7048^{*}$ & $0.6015^{* * *}$ & 0.5350 & $6.9723 * * *$ & 2.2002 & $2.2385 * * *$ & & $10.4617^{* * *}$ & $3.1516^{*}$ \\
\hline \multirow[t]{2}{*}{ leftturn } & 1 & $1.1286^{*}$ & 1.0693 & $.7974 * *$ & $0.6606^{*}$ & $1.2021 *$ & 1.3190 & 1.0634 & & 1.1575 & 0.8535 \\
\hline & 2 & $1.3267 * * *$ & $1.5196^{*}$ & $0.6885 * *$ & 0.8184 & $1.5403 * *$ & $2.0103 *$ & 1.0595 & & $3.8621 * * *$ & $2.0753^{*}$ \\
\hline plt & & $1.1531^{* *}$ & $1.3597^{*}$ & $1.2715^{* * *}$ & $1.4899 *$ & 1.1045 & $1.5067^{*}$ & 1.0033 & & $1.9071 * * *$ & $1.9600^{* *}$ \\
\hline fplt & & $1.2826^{* * * *}$ & $1.3686^{* *}$ & $1.9535^{* * *}$ & $2.2936^{* * *}$ & $0.6308 * * *$ & & 1.2384 & & $1.8436^{* * *}$ & 1.4781 \\
\hline pplt & & $1.1460 * *$ & $1.2783 *$ & $1.4745^{* * *}$ & $1.8103 * * *$ & $0.6365 * * *$ & 0.7202 & $1.6049 * * *$ & $2.2991 * * *$ & $2.0032 * * *$ & \\
\hline \multirow[t]{3}{*}{ speed 1} & 50 & 1.1518 & & $1.6721 * * *$ & 1.3996 & $1.5225^{* *}$ & & $0.5886^{*}$ & & 0.8718 & \\
\hline & 60 & 1.0990 & & 0.9185 & 0.7126 & $1.7733 * * *$ & & 0.8379 & & $0.6037 * *$ & \\
\hline & 70 & 1.1868 & & $2.4424 * * *$ & $2.3533 * * *$ & 0.9959 & & $0.4282 * * *$ & & $0.6667 *$ & \\
\hline \multirow[t]{3}{*}{ speed 2} & 50 & $1.5268 * * *$ & $1.7065^{* *}$ & $0.5207 * * *$ & $0.5768 *$ & & & $5.5721 * * *$ & $3.3765^{* *}$ & $4.3011 * * *$ & $4.7977 * * *$ \\
\hline & 60 & $1.5499 * * *$ & $1.7675 * * *$ & 0.9480 & 1.2572 & & & $3.3628 * * *$ & $2.4991^{*}$ & $3.9709 * * *$ & $4.0734 * *$ \\
\hline & 80 & 1.2026 & 1.5158 & $0.1096^{* * *}$ & $0.0644 * *$ & & & $15.5214^{* * *}$ & 3.9743 & 0.9361 & 1.0054 \\
\hline rlcinstalled2\#\#median & & $0.7628^{*}$ & & 0.7132 & & $0.5259 *$ & & & & $0.3385^{* * *}$ & \\
\hline rlcinstalled2\#\#channeliza- & & $1.3140^{* * *}$ & $1.2719 * *$ & $2.0445 * * *$ & $1.5862 * * *$ & $0.6473 *$ & & & & $1.8658^{* *}$ & \\
\hline \multirow[t]{2}{*}{ rlcinstalled2\#\#leftturn } & 1 & $0.7143 * * *$ & $0.7729 * *$ & $0.6812 * *$ & $0.6932 *$ & $1.8286^{* *}$ & & $0.5124 * * *$ & & $0.3915^{* * *}$ & \\
\hline & 2 & 0.9033 & 0.8813 & 0.7158 & $0.6874 *$ & 1.8592 & & 1.1301 & & $0.2362 * * *$ & \\
\hline \multirow[t]{2}{*}{ rlcinstalled2\#\#thrulanes } & 2 & 0.9662 & 1.0001 & & & 1.0436 & & $0.4962 *$ & & 1.4454 & \\
\hline & 3 & 1.0055 & 1.0710 & & & 1.5140 & & 0.5536 & & 0.9460 & \\
\hline
\end{tabular}




\begin{tabular}{|c|c|c|c|c|c|c|c|c|c|c|}
\hline 4 & $0.4257^{* * *}$ & $0.6013^{*}$ & & & $0.1844^{* * *}$ & & $0.1844 * * *$ & & $0.2753^{*}$ & \\
\hline rlcinstalled2\#\#plt & $0.7678 *$ & & & & $0.2004 * * *$ & & 0.7143 & & $0.5302 * *$ & \\
\hline rlcinstalled2\#\#plt & & & 1.3137 & & $0.3903 * *$ & & $0.5441 * *$ & & $2.9095^{* * *}$ & \\
\hline rlcinstalled2\#\#pplt & & & & & $0.1931^{* * *}$ & & & & & \\
\hline \multirow[t]{3}{*}{ rlcinstalled2\#\#speed 1} & 0.9116 & & & & & & $0.4288^{* *}$ & & & \\
\hline & $0.7828^{*}$ & & & & & & $0.3225^{* * *}$ & & & \\
\hline & 1.0962 & & & & & & 1.0164 & & & \\
\hline \multirow[t]{3}{*}{ rlcinstalled $2 \#$ \#speed 2} & & & & & & & & & & \\
\hline & & & & & & & & & & \\
\hline & & & & & & & & & & \\
\hline numcams & & & $1.0095^{* * *}$ & $1.0092^{* * *}$ & $0.9841^{* * *}$ & $0.9877 * * *$ & & & $1.0243^{* * *}$ & $1.0212^{* * *}$ \\
\hline timehalo & & & & & & & & & $1.2218^{*}$ & $1.1944 *$ \\
\hline timehalo2 & $1.0142 *$ & $1.0111^{*}$ & $1.0242 *$ & $1.0244 * *$ & & & & & & \\
\hline closestRLC0to500 & & & $0.40512 * * *$ & $0.4129 * * *$ & & & $1.6955^{* * *}$ & & & \\
\hline closestRLC500to 1000 & $1.2454 * * *$ & & $0.84199^{*}$ & & & & $1.4874 * *$ & & $1.4960^{* *}$ & \\
\hline Twoormore0to500 & $1.2498^{*}$ & & $3.856^{* * *}$ & $4.4274^{* * *}$ & & & $.4747 * * *$ & & & \\
\hline Twoormore500to 1000 & $0.4555^{* * *}$ & & & & $0.2345^{* * *}$ & & $0.3918^{* * *}$ & & $0.07438^{* * *}$ & \\
\hline Threewithin 1000 & & & $2.5960 * * *$ & $3.4619 * * *$ & & & & & $1.6357^{*}$ & \\
\hline cons & $0.0178^{* * *}$ & $0.1060^{* *}$ & $.00005^{* * *}$ & $.01233^{* * *}$ & 2.7013 & 2.5563 & $0.0082^{* * *}$ & 0.1393 & $0.00009^{* * *}$ & $0.0030^{* *}$ \\
\hline \multicolumn{11}{|c|}{ Goodness-of-fit Parameters } \\
\hline Observations & 685 & 685 & 685 & 685 & 685 & 685 & 685 & 685 & 685 & 685 \\
\hline Log-likelihood & -2131 & -2055 & -1632 & -1574 & -1411 & -1369 & -1357 & -1314 & -1012 & -994 \\
\hline $\operatorname{chi}^{2}$ & 464 & 160 & 701 & 284 & 260 & 73 & 300 & 50 & 334 & 106 \\
\hline AIC & 4328 & 4163 & 3323 & 3209 & 2879 & 2773 & 2775 & 2655 & 2090 & 2031 \\
\hline
\end{tabular}

Exponentiated coefficients $=* \mathrm{p}<0.05 * * \mathrm{p}<0.01{ }^{* * *} \mathrm{p}<0.001$ 
equipped intersections tend to have around $60 \%$ fewer rear-end collisions, but an increase of $70 \%$ of turning movement collisions. A RLC intersection within $500 \mathrm{~m}$ to $1 \mathrm{~km}$ away from a specific intersection reduced rear-end collisions by $15 \%$, but increases turning movement collisions by an estimated $48 \%$ and also increases sideswipe collisions movement by around $50 \%$. Therefore, evidence of collision migration was found for turning movement and sideswipe collisions.

The presence of two or more RLC intersections within a $500 \mathrm{~m}$ zone in relation to another intersection is estimated to reduce the turning movement collisions by $53 \%$ but massively increases the rear-end collisions by $380 \%$. This result can be expected as drivers will not take as many risks in making a turning movement knowing that there are several RLCs around and rear-end collisions would increase due to drivers stopping abruptly and the vehicle behind them not expecting them to stop. Having two or more RLCs within a $500 \mathrm{~m}$ to a $1 \mathrm{~km}$ distance seems to have better results on collision reductions, where there is an estimated decrease in angle collisions by $77 \%$, a reduction in turning movement collisions by $61 \%$ and a reduction in sideswipe collisions by $92 \%$. Other factors (not considered in this thesis) such as land-use around each specific intersection, local traffic management and driver negligence knowing that they have passed the RLC site may have been factors in increasing collisions at further intersections.

There was a minor, yet significant, time-halo effect evident in the rear-end, angle and sideswipe collisions. There was no apparent time-halo effect for turning movement collisions. As the number of RLCs increased throughout the city (numcams), the rear-end collisions have increased only negligibly at less than $1 \%$ and the sideswipe collisions increased by $2 \%$. The angle collisions on the other hand decreased by around $2 \%$. The 
specific years that have a set of RLCs installed (timehalo) seem to have an effect of increasing rear-end collisions by $2 \%$. The years after the installation years (timehalo2) seem to have an increase on the number sideswipe collisions of around $22 \%$. Therefore, when accounting for collisions due to time, the RLC effect overall seems to have a more affirmative safety effect in angle collision reductions but an increase in rear-end and sideswipe collisions.

Interaction effects from the NB regression models including the time and distance-halo variables also demonstrated that site-varying factors coupled with the RLC may significantly influence the collision frequencies by impact type. An updated summary of the significant interactions between the RLC and the different covariates for the collision impact types are presented in Table 5.9 based on the improved best fit models from this Section. The percentages of increase and decrease are obtained from the IRR values of the interaction effects from Table 5.8. They are almost identical to the effects presented in the previous section in Table 5.5. Variations in the results from the time and distance-halo variables were within a few percentages. Results of the interaction terms from the RENB spillover models can be found in Appendix I (Table C).

In summary, the significant reduction in collisions up to a distance of $1 \mathrm{~km}$ from RLC sites indicates that RLCs have a generalized effect on drivers' behaviors at intersections that extends beyond RLC. These results give confidence that spillovers exist and gives some sense of the mechanisms through which RLCs can operate. When these findings are not accounted for, the safety effects at the treatment sites and overall sites could be considerably underestimated. It is important to consider the geographic distribution of the RLC sites, as some of the sites were in close proximity to each other, 
Table 5.9 - Significant interactions from spillover models (nbreg)

\begin{tabular}{|c|c|c|c|c|c|}
\hline \multirow{2}{*}{ Variables } & Total & Rear-end & Angle & TM & Sideswipe \\
\hline & \multicolumn{5}{|c|}{ Determining effects of significant interactions with the RLC } \\
\hline rlcinstalled2 & 1 $6 \%$ & 1 $9 \%$ & $\sqrt{12 \%}$ & $\sqrt{9 \%}$ & 15\% \\
\hline median & & & $\sqrt{48 \%}$ & & $\sqrt{77 \%}$ \\
\hline channelization & $42 \%$ & 1. $39 \%$ & $\sqrt{46 \%}$ & & $86 \%$ \\
\hline leftturn & $\begin{array}{c}\text { - With } 1 \text { left- } \\
\text { turn lane } \\
\text { - With } 2 \text { left- } \\
\text { turn lanes } \\
17 \% \\
\text { (non- } \\
\text { significant) }\end{array}$ & $\begin{array}{c}\text { - With } 1 \text { left- } \\
\text { turn lane } \\
\text { - With } 2 \text { left- } \\
\text { turn lanes } \\
\sqrt{29 \%} \\
\text { (non- } \\
\text { significant) }\end{array}$ & $\begin{array}{l}\text { - With } 1 \text { left- } \\
\text { turn lane } \\
82 \% \\
\text { - With } 2 \text { left- } \\
\text { turn lanes } \\
85 \% \\
(\text { non- } \\
\text { significant })\end{array}$ & $\begin{array}{l}\text { - With } 1 \text { left- } \\
\text { turn lane } \\
49 \% \\
\text { - With } 2 \text { left- } \\
\text { turn lanes } \\
13 \% \\
\text { (non-significant) }\end{array}$ & $\begin{array}{l}\text { - With } 1 \text { left- } \\
\text { turn lane } \\
61 \% \\
\text { - With } 2 \text { left- } \\
\text { turn lanes } \\
77 \%\end{array}$ \\
\hline plt & & & $\sqrt{80 \%}$ & $\sqrt{75 \%}$ & $\sqrt{43 \%}$ \\
\hline fplt & $21 \%$ & & $\sqrt{71 \%}$ & $\sqrt{75 \%}$ & \\
\hline pplt & & & $81 \%$ & $\sqrt{60 \%}$ & \\
\hline speed1 & $\begin{array}{c}\text { - At } 50 \mathrm{~km} / \mathrm{hr} \\
\text { speed limit } \\
18 \% \\
\text { - At } 60 \mathrm{~km} / \mathrm{hr} \\
\text { speed limit } \\
28 \% \\
\text { - At } 70 \mathrm{~km} / \mathrm{hr} \\
\text { speed limit } \\
\sqrt[2]{2 \%}\end{array}$ & & & $\begin{array}{c}\text { - At } 50 \mathrm{~km} / \mathrm{hr} \\
\text { speed limit } \\
58 \% \\
\text { - At } 60 \mathrm{~km} / \mathrm{hr} \\
\text { speed limit } \\
68 \% \\
\text { - At } 70 \mathrm{~km} / \mathrm{hr} \\
\text { speed limits } \\
<<1 \%\end{array}$ & \\
\hline \multicolumn{6}{|l|}{ speed2 } \\
\hline thrulanes & $\begin{array}{l}\text { - With } 2 \text { thru } \\
\text { lanes } \\
\text { (non-significant) } \\
\text { - With } 3 \text { thru } \\
\text { lanes } 5 \% \\
\text { (non-significant) } \\
\text { - With } 4 \text { thru } \\
\text { lanes } 56 \%\end{array}$ & & $\begin{array}{l}\text { - With } 2 \text { thru } \\
\begin{array}{l}\text { lanes } \\
\text { (non-significant) }\end{array} \\
\text { - With } 3 \text { thru } \\
\begin{array}{l}\text { lanes } 51 \% \\
\text { (non-significant) }\end{array} \\
\text { - With } 4 \text { thru } \\
\text { lanes } / 82 \%\end{array}$ & $\begin{array}{l}\text { - With } 2 \text { thru } \\
\text { lanes } \\
\text { - With } 3 \text { thru } \\
\text { lanes } 45 \% \\
\text { - With } 4 \text { thru } \\
\text { lanes } 82 \% \\
\text { (non-significant) }\end{array}$ & $\begin{array}{l}\text { - With } 2 \text { thru } \\
\text { lanes } 44 \% \\
\text { - With } 3 \text { thru } \\
\text { lanes } 6 \% \\
\text { - With } 4 \text { thru } \\
\text { lanes } 73 \%\end{array}$ \\
\hline
\end{tabular}

possibly reducing their effectiveness. A model that explicitly incorporates spillover effects is important for forecasters to evaluate the impact of wider benefits on policy. 


\subsection{Empirical Bayes Evaluation}

The average collision frequency of comparison sites and the variation around this average are brought into the EB procedure by the SPF models, using trend data from all the nonRLC intersections. The SPF models represent the change in mean collision frequency as AADT increases or decreases. These models estimate what the average safety performance (in yearly collision frequency) is expected to be at a group of typical signalized intersections representing a range of traffic volumes. A negative binomial model was applied to estimate the SPFs and the results are presented in Table 5.10, following examples from Hauer et al. (2001), Council et al. (2005), Hagen (2014), and Weber (2015).

Table 5.10 - SPF models (calculated from full set of non-RLC intersections)

\begin{tabular}{|c|c|c|c|c|c|}
\hline Model & Variable & Coefficient & Std. Error & $\begin{array}{c}\text { Over- } \\
\text { dispersion } \\
\text { parameter } \\
\end{array}$ & SPF Model Form \\
\hline \multirow{2}{*}{ Total } & $A A D T$ & 4.54E-05 & $5.07 \mathrm{E}-06$ & \multirow{2}{*}{0.1819} & \multirow{2}{*}{$5.62 \mathrm{E}-01 * A A D T^{\wedge} 4.54 \mathrm{E}-05$} \\
\hline & Intercept & $5.62 \mathrm{E}-01$ & $1.71 \mathrm{E}-01$ & & \\
\hline \multirow{2}{*}{ Rear-end } & $A A D T$ & $6.56 \mathrm{E}-05$ & $6.53 \mathrm{E}-06$ & \multirow{2}{*}{0.1589} & \multirow{2}{*}{$-1.07 * A A D T^{\wedge} 6.56 \mathrm{E}-05$} \\
\hline & Intercept & $-1.07 \mathrm{E}+00$ & $2.41 \mathrm{E}-01$ & & \\
\hline \multirow{2}{*}{ Angle } & $A A D T$ & $2.48 \mathrm{E}-05$ & $7.82 \mathrm{E}-06$ & \multirow{2}{*}{0.1692} & \multirow{2}{*}{$-3.54 \mathrm{E}-01 * A A D T^{\wedge} 2.48 \mathrm{E}-05$} \\
\hline & Intercept & $-3.54 \mathrm{E}-01$ & $2.66 \mathrm{E}-01$ & & \\
\hline \multirow{2}{*}{$\begin{array}{l}\text { Turning } \\
\text { Movement }\end{array}$} & $A A D T$ & $2.90 \mathrm{E}-05$ & $8.04 \mathrm{E}-06$ & \multirow{2}{*}{0.2182} & \multirow{2}{*}{$-4.17 \mathrm{E}-01 * A A D T^{\wedge} 2.90 \mathrm{E}-05$} \\
\hline & Intercept & $-4.17 \mathrm{E}-01$ & $2.73 \mathrm{E}-01$ & & \\
\hline \multirow{2}{*}{ Sideswipe } & $A A D T$ & $2.59 \mathrm{E}-05$ & $6.81 \mathrm{E}-06$ & \multirow{2}{*}{0.3908} & \multirow{2}{*}{$-5.91 \mathrm{E}-01 * A A D T^{\wedge} 2.59 \mathrm{E}-05$} \\
\hline & Intercept & $-5.91 \mathrm{E}-01$ & $2.80 \mathrm{E}-01$ & & \\
\hline
\end{tabular}

For total collisions, the estimate of the constant regression parameter is 0.5621 , and the estimate of the AADT regression parameter 4.54E-05. This means that at an intersection with AADT of 40,000 vehicles per day, then one should expect $0.562 \times 40000^{\wedge} 4.54 \mathrm{E}-05=0.5623$ collisions/(intersection-year). Here AADT plays the role of one trait value, no additional trait values are represented in the SPF. The AADT 
was the only factor used in the model following Hauer (2001) and the Synectics (2003) report conducted for intersections in Ontario, which included Ottawa. However in that report the effect of the RLC treatment was combined with police enforcement.

Next, based on the SPF models developed in Table 5.10 and the over-dispersion parameters derived from those models, the number of expected collisions had the RLC treatment not been installed was estimated using the weighted adjustment equations (Eq. 3.5 and 3.6) on the predictive model estimate, as discussed earlier in Section 3.1.2. Detailed results of the different collision impact types for each individual intersection along with the equations used are presented in Appendix $\mathrm{J}$.

Results in Table 5.11 presents summaries of the evaluation of the effect of the RLC treatment based on the percentage differences between the expected number of collisions had the treatment not been installed and the observed post-treatment number of collisions averaged over the 34 RLC sites. This procedure accounts for regression-to-themean effects that result from the natural tendency to select for treatment those sites with high observed crash frequencies.

Table 5.11 - Expected number of collisions from the calculated SPFs

\begin{tabular}{|l|r|r|r|r|}
\hline $\begin{array}{c}\text { Collision Impact } \\
\text { Type/Classification }\end{array}$ & $\begin{array}{c}\text { AAOC } \\
\text { (Before } \\
\text { RLC) }\end{array}$ & $\begin{array}{c}\text { AAOC } \\
\text { (After } \\
\text { RLC) }\end{array}$ & $\begin{array}{c}\text { Expected collisions } \\
\text { AFTER if RLC were not } \\
\text { installed (EB model } \\
\text { calculation) }\end{array}$ & \% $^{\text {change }}$ \\
\hline \hline Total Collisions & 514 & 513 & 503 & 194 \\
\hline Rear-end & 192 & 230 & 147 & $\mathbf{1 . 8 \%}$ \\
\hline Angle & 142 & 95 & 106 & $\mathbf{- 5 3 . 7 \%}$ \\
\hline Turning movement & 101 & 87 & 49 & $\mathbf{- 2 2 . 2 \%}$ \\
\hline Sideswipe & 46 & 62 & $\mathbf{2 0 . 9 \%}$ \\
\hline
\end{tabular}

${ }^{\mathrm{a}} \mathrm{AAOC}=$ Average annual observed collisions for 34 RLC intersections.

${ }^{\mathrm{b}}$ Percent change between AAOC after RLC and effect without RLC.

Models based on the collision impact types show a reduction in angle collisions by $53 \%$ and a reduction in turning movement collisions by $22 \%$. On the other hand, total 
collisions increased by just over $1 \%$ and rear-end collisions also increased by $15 \%$.

The accuracy of the developed models and results are subject to the characteristics of the non-RLC intersections adopted in this thesis. It has to be noted that the difference in the estimation of the SPF model and the dispersion parameter can produce different results when the EB procedure is applied. Since collision patterns usually vary in different geographical areas, the SPFs must be calibrated to reflect local conditions. Nontreatment sites should be chosen that have similar characteristics to the RLC sites. These models can also be transferred to other cities by recalibrating and checking the results against known points in the new city to develop a multiplier (Bissonette and Cramer 2008). This recalibration will adapt the model to local conditions. The estimated model and results from these analyses can assist decision makers in the transportation area to allocate funds on safety improvements that could have the most benefit in terms of RLC collision reduction.

\subsection{Case-Control Evaluation}

In the first step of the PSM, the logit model of Eq. (3.11) was estimated using StataSE 14.2 statistical software's user-written command psmatch2 which includes algorithms for common support graphing and covariate imbalance testing (pstest) (Leuven and Sianesi, 2003) in order to estimate the propensity scores. Here the propensity score is the probability that the intersection received the RLC treatment. With matching, different sets of variables were tested and the one with the best balance was chosen. After the confirmation of the balance of covariates, the ATT was estimated using the StataCorp (2013) teffects command. Detailed output of all the models run can be found in Appendix $\mathrm{K}$. 
Although the teffects package constructs a propensity score and calculates a treatment effect with a one-line command, it does not present details regarding the propensity scores. Hence, the psmatch 2 command was carried out first in order to compare covariate distributions across our matched groups to ensure that adequate balance has been obtained before calculating treatment effects.

A logit regression was run with the RLC treatment as the outcome variable and the potential confounders as explanatory variables. Next, the StataSE software matches the RLC and non-RLC intersections based on their propensity scores for each observation. To check the region of common support, which is the overlap in range of propensity scores across treatment and comparison groups, a visual inspection of the frequency distributions of the propensity scores for the RLC and non-RLC intersections before and after matching is shown in Figure 5.3.

The overlap indicates that observations from both groups are available across the range of the propensity score, making it possible to perform reasonable comparisons. The area of overlap identifies intersections that have comparable propensity scores among treated and untreated subjects, and are therefore better candidates for inclusion in a comparative analysis. The results from the common support show that seven treatment observations were not used. These are the ones that no good matches were found for, the closest control case for each of them had a propensity score that was more than 0.01 away from them. So these results are based on the 192 treatment cases for which there were good matches. Conversely, the amount of non-overlap of these two curves identifies intersections who have a very low probability of treatment and are never treated. Detailed results of the output from StataSE are found in Appendix K. 

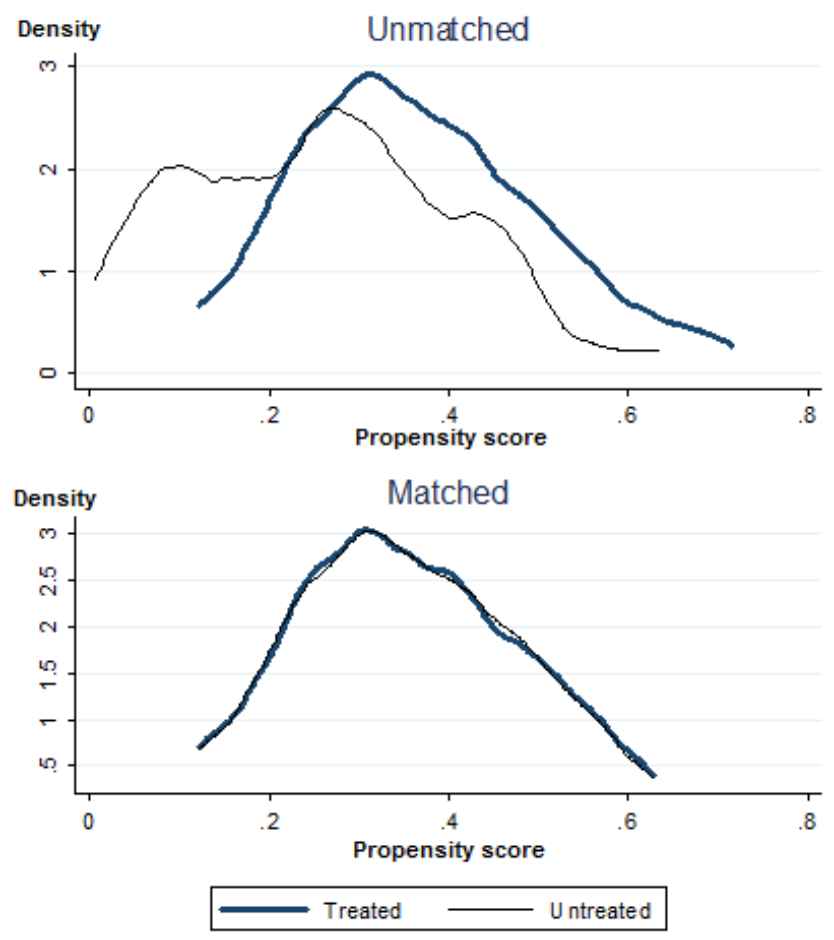

Figure 5.3 - Distribution and matching of the propensity scores estimation between RLC and non-RLC intersections for total collisions

Next, the matching procedure was checked to see if the propensity score across the groups was able to balance the distribution of the relevant covariates in both the treatment and control group. A visual representation for checking the covariate imbalance is shown in Figure 5.4 to determine if the matching made the treatment and control groups of covariates more alike.

In order to determine whether the propensity score successfully balanced the data on observed covariates, the proposed maximum standardized differences for specific covariates range from 10 to 25 percent is considered a balance (Austin, 2009; Stuart et al., 2013). There is no rule regarding how much imbalance is acceptable in a propensity score. 


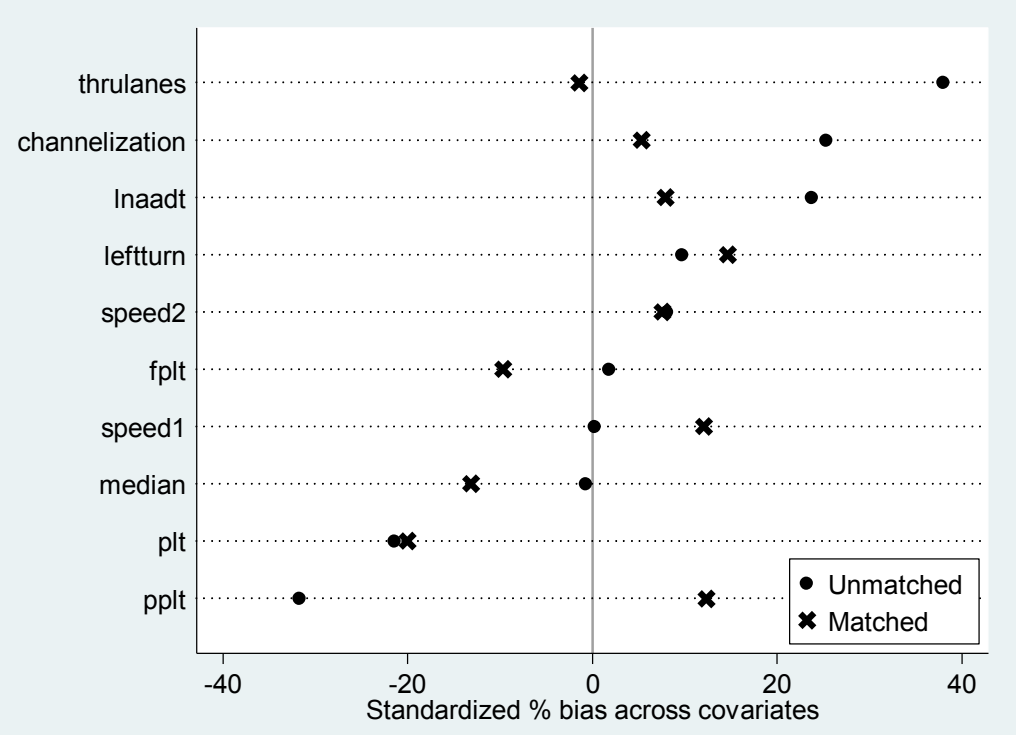

Figure 5.4 - Percent standardized bias of covariates before and after matching

As seen in Figure 5.4, the propensity score test showed a significant reduction in bias in the majority of covariates after matching. If a variable is thought to be related to the outcome but not the treatment, including it in the propensity score should reduce the overall bias (Brookhart et al., 2006; Austin, 2011). This expectation is because there is a chance that a variable related to the outcome is also related to treatment. If it is not accounted for in the propensity score, it is an unmeasured confounder and will bias the treatment effect (Brookhart et al., 2006). Therefore, it is beneficial to include all variables that are potentially related to the outcome and evaluate the balance by the overall model standardized mean bias before and after matching (Garrido et al., 2014). The mean bias of the overall model was $16.1 \%$ in the unmatched model and $9.8 \%$ in the matched model, indicating a good match.

Finally, to estimate the ATT of the RLCs on the different collision impact types, the teffects ipwra StataSE command was used as a doubly-robust estimation. The estimation algorithm is built up of two parts: (1) a logit model to predict treatment status 
as a function of number of total collisions, angle collisions, rear-end collisions, and turning movement collisions (which can be found in Appendix $\mathrm{K}$ for each collision impact type under the StataSE psmatch2 command), (2) a linear regression to model the outcome of each collision type, using the explanatory variables. This method guards against a misspecification of the propensity score. It performs multivariable regression analysis on a sample weighted by the propensity score. As long as either the propensity score or the regression model is specified correctly, the treatment effect estimates will not be biased (Allison, 2015). The results of the estimated ATT are presented in Table 5.12.

Table 5.12 - Impact of RLC (ATT) from PSM method on different collision impact types

\begin{tabular}{|c|c|c|c|c|c|c|c|}
\hline $\begin{array}{l}\text { Collision } \\
\text { impact type }\end{array}$ & $\begin{array}{l}\text { ATT } \\
\text { coef. }\end{array}$ & $\begin{array}{l}\text { Std. } \\
\text { Err. }\end{array}$ & $\mathbf{z}$ & $\mathbf{P}>\operatorname{chi}^{2}$ & \multicolumn{2}{|c|}{ [95\% Conf. Interval] } & $\begin{array}{l}\text { Balance test } \\
\text { (tebalance }\end{array}$ \\
\hline $\begin{array}{l}\text { Total collisions } \\
\text { with spillover } \\
\text { variables }\end{array}$ & -1.8096 & 0.8185 & -2.21 & 0.0270 & -3.4139 & -0.2052 & 0.1829 \\
\hline Total collisions & -0.3629 & 0.6144 & -0.59 & 0.5550 & -1.5671 & 0.8413 & 0.1829 \\
\hline Rear-end & 0.8366 & 0.4379 & 1.91 & 0.0560 & -0.0217 & 1.6948 & 0.6555 \\
\hline Angle & -0.7164 & 0.2601 & -2.75 & 0.0060 & -1.2262 & -0.2066 & 0.1719 \\
\hline $\begin{array}{l}\text { Turning } \\
\text { movement }\end{array}$ & -0.7058 & 0.2450 & -2.88 & 0.0040 & -1.1860 & -0.2257 & 0.1401 \\
\hline Sideswipe & 0.1231 & 0.1953 & 0.63 & 0.5290 & -0.2598 & 0.5059 & 0.2142 \\
\hline
\end{tabular}

The results of the total number of collisions indicate that if all intersections had RLCs there would be 0.3629 collisions less than the average of collisions that would occur if none of the intersections had RLCs. However, the estimation is non-significant. The impact of the of RLCs on reducing collisions in angle an turning movement impacts was significant reductions of 0.7164 and 0.7058 , respectively. On the other hand, intersections with RLCs had a significant increase in rear-end collisions by 0.8366 , and a non-significant slight increase in sideswipe collisions of 0.1231 . 
Imai and Ratkovic (2014) derived a test for balance by viewing the restrictions imposed by balance as over-identifying conditions. This test was implemented using thetebalance overid command in StataSE, which tests whether the above treatment models balanced the covariates for all the collision impact types. This balance test uses the $\mathrm{chi}^{2}$ test for balance. When the Prob $>\mathrm{chi}^{2}$ is greater than 0.05 the null hypothesis cannot be rejected that the IPWRA model balanced all covariates (Stata Manual, 2016). The tebalance overid estimates were all insignificant after matching indicating that no systematic differences remained in the distribution of covariates between the RLC and non-RLC intersections after matching, which means the models are balanced.

The ATT coefficients were used to estimate the percent change of collisions due to the RLC effect. The results are presented in Table 5.13, indicating a decrease of $19 \%$ in angle collisions and a decrease of $21 \%$ in turning movement collisions and an increase rear-end collisions and sideswipe collisions by $12 \%$ and $7 \%$ respectively. The total collisions seems to decrease by $2 \%$ here since the decrease in angle and turning movement exceed the increase in rear-end and sideswipe. Results are in line with the findings of the other approaches explored in the previous sections.

In summary, this novel technique (the PSM) has a number of advantages: (1) the derivation of the propensity score in the absence of outcome data avoids the potential for inappropriate skewing of estimates of the treatment effect on outcome during the development of a multivariable model. (2) The PSM can also avoid modeling assumptions involving extrapolations that may occur when the joint distribution of covariates has substantial differences between treatment groups. (3) In instances where the outcome is rare compared to the baseline covariates (i.e. if modeling fatal collisions) 
Table 5.13 - Impact of RLC (PSM ATT) in terms of percentage of collision reduction

\begin{tabular}{|c|c|c|c|c|c|}
\hline \multirow[t]{2}{*}{ Collision Impact Type } & $\begin{array}{c}\text { AAOC }^{\mathrm{a}} \\
\text { (Before RLC) }\end{array}$ & $\begin{array}{c}\text { AAOC }^{a} \\
\text { (After RLC) }\end{array}$ & $\begin{array}{c}\text { ATT } \\
\text { Coef. }^{\text {b }} \\
\end{array}$ & $\begin{array}{c}\text { Effect } \\
\text { without RLC } \\
(2)-(3) \\
\end{array}$ & $\begin{array}{c}\text { Percent } \\
\text { Change } \\
(2)-(4) /(4) \\
\end{array}$ \\
\hline & (1) & (2) & (3) & (4) & (5) \\
\hline $\begin{array}{l}\text { Total collisions with } \\
\text { spillover variables }\end{array}$ & 14.18 & 16.72 & -1.8096 & 18.5296 & $-9.8 \%$ \\
\hline Total collisions & 14.18 & 16.72 & -0.3629 & 17.0829 & $-2.1 \%$ \\
\hline Rear-end & 5.00 & 7.88 & 0.8366 & 7.0434 & $11.9 \%$ \\
\hline Angle & 3.82 & 3.08 & -0.7164 & 3.7964 & $-18.9 \%$ \\
\hline Turning movement & 3.03 & 2.69 & -0.7058 & 3.3958 & $-20.8 \%$ \\
\hline Sideswipe & 1.35 & 1.96 & 0.1231 & 1.8369 & $6.7 \%$ \\
\hline
\end{tabular}

${ }^{\mathrm{a}} \mathrm{AAOC}=$ Average annual observed collisions per intersection.

${ }^{\mathrm{b}}$ ATT coefficients of PSM models from Table 5.12.

and the sample size is not sufficient to take into account the multiple covariates, propensity score methods may be more appropriate.

\subsection{Comparison of Results from the Statistical Methods}

A comparison of results from the previous five sections (Section 5.1-5.5) was carried out to find out if there are any major differences in the results obtained from the different statistical methods applied. The summary of the RLC effect from each method in presented Table 5.14 indicates that while the estimated counterfactual number of collisions varied slightly across the different statistical methods, the direction and magnitude of the RLC effect were similar and consistent for the most part.

Table 5.14 - Summary of the results from the different statistical approaches

\begin{tabular}{|l|r|r|r|r|r|r|}
\hline \multirow{2}{*}{$\begin{array}{c}\text { Collision Impact } \\
\text { Type }\end{array}$} & NB & \multicolumn{6}{|c|}{ Estimated Effect of RLCs on Collisions } \\
\cline { 2 - 7 } & RENB & $\begin{array}{c}\text { RENB w/ } \\
\text { Interaction } \\
\text { variables }\end{array}$ & $\begin{array}{c}\text { NB w/ } \\
\text { interaction } \\
\text { \& spillover } \\
\text { variables }\end{array}$ & \multirow{2}{*}{ EB } & PSM \\
\hline \hline Total Collisions & $0.9 \%$ & $0.01 \%$ & $-0.3 \%$ & $5.6 \%$ & $1.8 \%$ & $-2.1 \%$ \\
\hline Rear-end & $17.9 \%$ & $23.8 \%$ & $1.8 \%$ & $8.8 \%$ & $15.7 \%$ & $11.9 \%$ \\
\hline Angle & $-16.8 \%$ & $-28.5 \%$ & $-14.8 \%$ & $-11.7 \%$ & $-53.8 \%$ & $-18.9 \%$ \\
\hline Turning movement & $-16.3 \%$ & $-14.0 \%$ & $-18.1 \%$ & $-8.7 \%$ & $-22.2 \%$ & $-20.8 \%$ \\
\hline Sideswipe & $18.5 \%$ & $34.5 \%$ & $14.3 \%$ & $15.4 \%$ & $20.9 \%$ & $6.7 \%$ \\
\hline
\end{tabular}

Note: A negative sign indicates a reduction in collisions. 
In addition to demonstrating the value of using multiple evaluation methods, these results show the effects and potential value of using RLCs to affect collision profiles for critical intersections. The different methods all indicate that while RLCs mainly reduce the overall collision frequencies modestly, this aggregate effect is the result of a substantial decrease in angle and turning-movement collisions that is partly offset by an increase in rear-end and sideswipe collisions. From a safety perspective, therefore, RLCs can be seen as a valuable instrument for managing traffic safety problems in urban areas.

The consistency in results is an important finding confirming that the PSM methodology appears to be a viable alternative to the EB methodology, which is the current method most commonly used in traffic safety assessments. It is important to note that there is a difference in the data requirement for the EB and PSM methods. Although both methods rely on a large sample of untreated control sites, the EB method further requires that the untreated sites must be representative of the treated sites in order to estimate the SPFs, which constrains the application of the EB method. Using multiple modeling techniques that address the different deficiencies in the different methods adds to the robustness and confidence in the evaluation results of the different traffic-related treatments. A summary of the different approaches and what they adjust for is presented in Table 5.15.

Therefore, it is suggested that when estimating treatment effects in future research using observational data, the PSM method is preferred to regression-based methods for several practical reasons:

(1) Unlike regression-based methods, where the researcher is usually tempted to continually modify the regression model to achieve the desired outcome, in the PSM 
Table 5.15 - What the different statistical methods adjust for

\begin{tabular}{|l|c|c|}
\hline \multicolumn{1}{|c|}{ Statistical Method } & $\begin{array}{c}\text { Adjustment for } \\
\text { Selection Bias }\end{array}$ & $\begin{array}{c}\text { Adjustment for Other } \\
\text { Covariates }\end{array}$ \\
\hline \hline RLC effect on outcome in naive before-after analysis & No & No \\
\hline $\begin{array}{l}\text { Regression of RLC effect on outcome and control } \\
\text { variables in NB modeling }\end{array}$ & No \\
\hline $\begin{array}{l}\text { Regression of RLC effect on outcome and control } \\
\text { variables in EB modeling }\end{array}$ & Yes & $\begin{array}{c}\text { Yes } \\
\text { (only when control } \\
\text { variables are used) }\end{array}$ \\
\hline $\begin{array}{l}\text { Propensity score matched sample, doubly-robust } \\
\text { method (regression of RLC effect on outcome and } \\
\text { covariates within sample matched by propensity score } \\
\text {-teffects ipwra-) }\end{array}$ & Yes & Yes \\
\hline
\end{tabular}

method the propensity score is estimated and a matched sample is constructed without any reference to the outcome.

(2) In the PSM method, the outcomes are explicitly compared between treated and untreated subjects that have a similar distribution of observed baseline covariates. In the regression-based methods, the degree of overlap between the distributions of baseline covariates for the two groups may be difficult to assess. In a setting in which there is a strong separation between the two groups, a naïve analyst may proceed with a regressionbased analysis without being aware that the fitted regression model is interpolating between two distinct populations. While in the PSM method, if there are substantial differences in baseline covariates between treated and untreated subjects, it restricts the analysis to comparing outcomes between the minority of treated and untreated subjects who have similar covariate patterns. Or the analysis can get discontinued concluding that treated and untreated subjects are so different that a meaningful comparison of outcomes between the two groups is not plausible.

(3) It is simpler to determine whether the propensity score model has been adequately specified conditional on the propensity score, but much more difficult to 
determine whether the regression model has been correctly specified (Austin, 2011). To assess whether the propensity score model has been adequately specified, the distribution of measured baseline covariates is compared between treated and untreated subjects. In contrast, it is much more difficult to determine whether the regression model relating treatment selection and baseline covariates to the outcome has been correctly specified. Goodness-of-fit measures do not allow one to determine the degree to which the fitted regression model has successfully eliminated systematic differences between treated and untreated subjects. Thus, regression models are unable to reconstruct counterfactuals as accurately.

Finally, the greater analytical flexibility and confidence that can be acquired through multiple assessment methods can also help to identify combinations of treatments that can enhance the safety benefits of different traffic management techniques. By examining more specifically the conditions in which RLCs are associated with certain types of collisions, policy-makers can combine treatments to create the best conditions for reducing collision frequencies by impact type. 


\section{Economic Evaluation: City of Ottawa Case Study}

This chapter presents results of the economic analysis of the RLCs. Section 6.1 presents the benefit calculations details. Section 6.2 present results of the benefit-cost models. The objective of a benefit-cost analysis is to quantifying the RLC program costs versus the economic safety benefits to determine the return on road safety investment.

\subsection{Benefit-Cost Model}

The last objective used to gauge the success of the RLC implementation is to assess whether it achieves the desired return on investment. To determine this, the NPV and BCR are calculated according to Equation 3.9 and 3.10. The first step in calculating the $\mathrm{NPV}$ and the BCR is to convert the treatment effect into an annualized reduction (or increase) in collisions, based on the statistical analysis results performed in Chapter 5. The reductions (or increases) are then converted into annual benefits (or detriments) using average collision cost values.

To do this, the frequency of the collision impact types and severities were combined into one cost analysis to determine the overall observed effect of RLCs. The observed frequency of collisions and the estimated reduction or increase in frequency of collisions based on the effectiveness of the RLC (obtained from the PSM method from Table 5.13) were multiplied by the social collision costs by collision severity. The costs were annualized for the calculation of net benefit per year. Results are presented in Table 6.1.

The annual total safety benefit for the RLC program is calculated by summarizing the products of the number of fatal and injury collisions reduced by the 
Table 6.1 - Annual cost of collisions by impact type

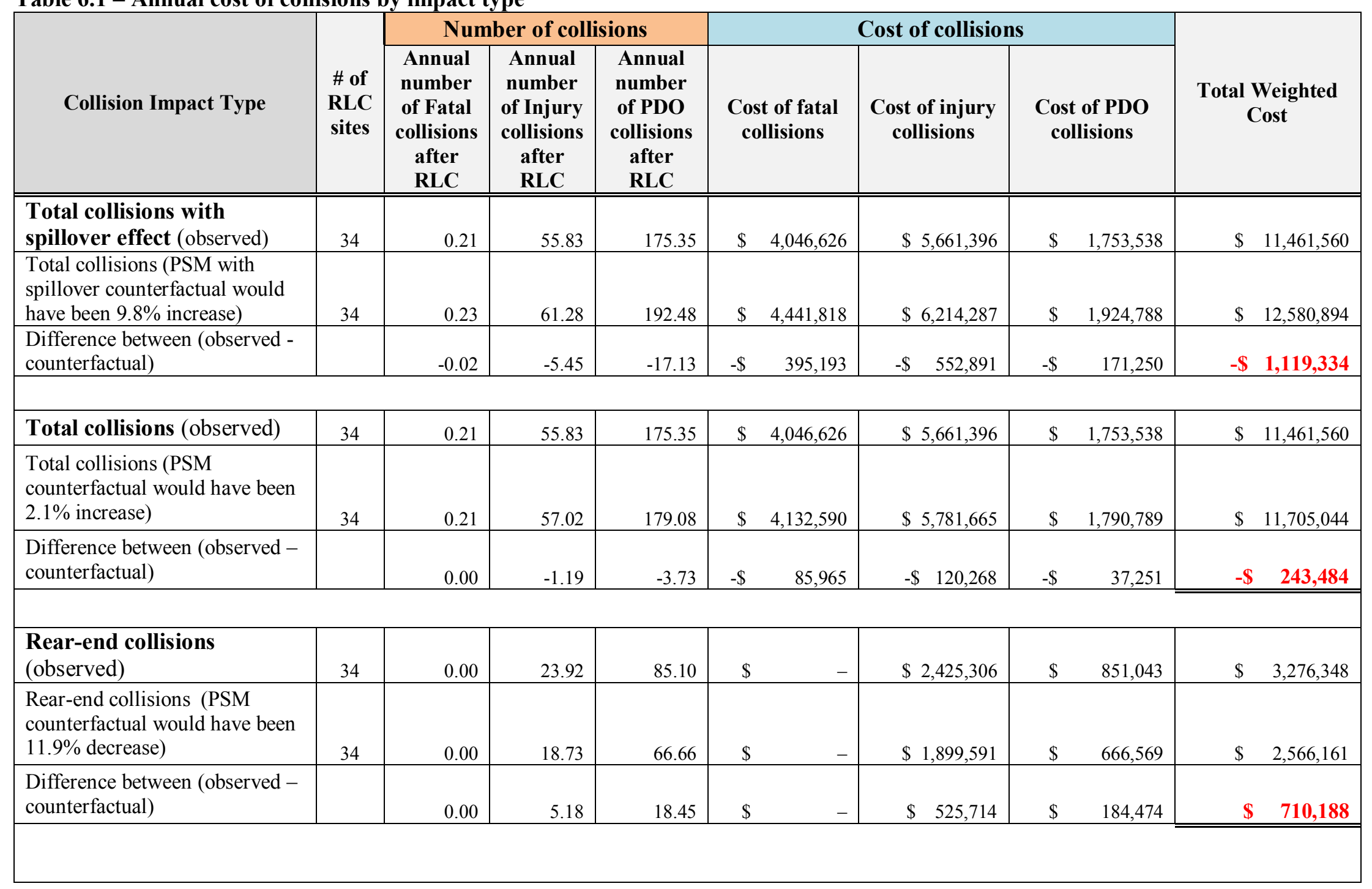




\begin{tabular}{|c|c|c|c|c|c|c|c|c|c|c|c|}
\hline Angle collisions (observed) & 34 & 0.21 & 13.91 & 28.51 & $\$$ & $4,046,626$ & $\$ 1,410,061$ & $\$$ & 285,072 & $\$$ & $5,741,759$ \\
\hline $\begin{array}{l}\text { Angle collisions (PSM } \\
\text { counterfactual would have been } \\
18.9 \% \text { increase) }\end{array}$ & 34 & 0.26 & 17.06 & 34.97 & $\$$ & $4,963,410$ & $\$ 1,729,518$ & $\$$ & 349,656 & $\$$ & $7,042,585$ \\
\hline $\begin{array}{l}\text { Difference between (observed - } \\
\text { counterfactual) }\end{array}$ & & -0.05 & -3.15 & -6.46 & $-\$$ & 916,785 & $-\$ \quad 319,457$ & $-\$$ & 64,584 & $-\$$ & $1,300,826$ \\
\hline $\begin{array}{l}\text { Turning movement } \\
\text { collisions (observed) }\end{array}$ & 34 & 0.00 & 11.47 & 25.73 & $\$$ & - & $\$ 1,163,301$ & $\$$ & 257,260 & $\$$ & $1,420,560$ \\
\hline $\begin{array}{l}\text { Turning movement collisions } \\
\text { (PSM counterfactual would } \\
\text { have been } 20.8 \% \text { increase) }\end{array}$ & 34 & 0.00 & 13.86 & 31.07 & $\$$ & - & $\$ 1,405,100$ & $\$$ & 310,733 & $\$$ & $1,715,833$ \\
\hline $\begin{array}{l}\text { Difference between (observed - } \\
\text { counterfactual) }\end{array}$ & & 0.00 & -2.38 & -5.35 & $\$$ & - & $-\$ 241,800$ & $-\$$ & 53,473 & $-\$$ & 295,273 \\
\hline $\begin{array}{l}\text { Sideswipe collisions } \\
\text { (observed) }\end{array}$ & 34 & 0.00 & 1.32 & 25.87 & $\$$ & - & $\$ 133,956$ & $\$$ & 258,650 & $\$$ & 392,606 \\
\hline $\begin{array}{l}\text { Sideswipe collisions (PSM } \\
\text { counterfactual would have been } \\
6.7 \% \text { decrease) }\end{array}$ & 34 & 0.00 & 1.23 & 24.13 & $\$$ & - & $\$ 124,980$ & $\$$ & 241,319 & $\$$ & 366,299 \\
\hline $\begin{array}{l}\text { Difference between (observed - } \\
\text { counterfactual) }\end{array}$ & & 0.00 & 0.09 & 1.73 & $\$$ & - & 8,976 & $\$$ & 17,331 & $\$$ & 26,307 \\
\hline
\end{tabular}


RLC program, multiplied by the corresponding percent reduction/increase value for the collision type based on the statistical analysis results. The results revealed that from a positive aggregate economic benefit the annual safety benefit of the RLC program (34 sites) approximated a net cost reduction of $\$ 1,119,334$. It is estimated that the RLC program in Ottawa prevents 5.5 injury collisions and 17.1 PDO collisions annually.

The collision reduction benefits include the avoided collision costs associated with a $19 \%$ reduction in angle collisions (savings $>\$ 1,300,000$ ), a $21 \%$ reduction in turning movement collisions (savings $>\$ 295,000$ ) and the revenue from issued citations (\$2.2million). However, the RLC presence is estimated to increase the rear-end collision frequencies by $25 \%$ (a loss $>\$ 710,000$ ) and sideswipe collisions by $7 \%$ (a loss $>\$ 26,000$ ).

Tanaka said, "Red-light cameras are known to increase the incidence of rear-end collisions, but that those crashes are less likely to be serious than right-angle collisions, which red-light cameras help prevent." (Tumilty, 2017). Unless there are specific records indicating the severity level of the injury and PDO of every collision and by victim, this is a critical assumption made by the Program Manager of road safety and traffic investigations in Ottawa. The cost estimation results in Table 6.1 shows otherwise when breaking down the costs of the angle and rear-end collisions by the collision classification. Beyond a collision being fatal, danger lie in the types of injuries a person may suffer. During rear-end collisions, a person could also suffer whiplash, broken bones, sprains and strains, head trauma, cuts, bruising and more.

The assumption that all angle collisions are more severe then rear-end collisions would require assigning a cost to each level of injury, then subdividing the combined injury category in more detail. According to the data stored by the City of Ottawa, 
records show that collision severities are recorded for the overall collision and not by victim. The three general categories recorded are: (1) Fatal (2) Non-fatal injury and (3) PDO.

When strictly looking at the cost of injury collisions and the cost of PDO collisions in Table 6.1, results indicate that there is a major increase in the observed rearend collision costs (due to the increase in frequency) that do not negate the savings in angle collision costs. Rear-end collisions have around double the frequency of injury collisions than angle collisions and almost triple the frequency of PDOs. What creates a major difference in cost between these two collision impact types is the fatal collisions. As discussed earlier in Section 4.4.2, due to the small numbers of fatal collisions related to the angle collisions, a small change in the total count of these types of collisions could have a significant effect on outcomes due to their high social cost.

Next, the costs of installing and operating RLCs were contrasted with the monetary value of benefits brought about by collision reduction and prosecution, and the results are shown in Table 6.2. Scenarios 1 and 2 present the benefits of collision reduction based on the total number of collisions without considering the spillover effects and Scenarios 3 and 4 consider the spillover effects. Scenario 3 and 4 will be discussed further since they presented a better model fit with the spillover terms (from Section 5.5).

In terms of net benefits, Scenario 3 presents the cost savings from the reduction in the total number of collisions at RLC intersections (including the spillover effects) plus the fine revenue minus the RLC annual costs which yields $\$ 4.4$ million in savings. Had the fine revenue not been considered as part of the savings, the RLC annual costs would have exceeded the savings by over $\$ 148,000$, as presented in Scenario 4 of Table 6.2. 
Table 6.2 - Summary of social costs and benefits

\begin{tabular}{|c|r|r|r|r|r|}
\hline Scenario & $\begin{array}{c}\text { Annual } \\
\text { RLC costs }\end{array}$ & $\begin{array}{c}\text { Annual fine revenue } \\
\text { (from Table 4.8) }\end{array}$ & $\begin{array}{c}\text { Collision } \\
\text { reduction } \\
\text { benefit } \\
\text { from Table 6.1) }\end{array}$ & Net benefits & BCR \\
\hline \hline Scenario 1 & $\$ 1,267,641$ & $-\$ 4,591,080$ & $-\$ 243,484$ & $-\$ 3,566,923$ & 3.81 \\
\hline Scenario 2 & $\$ 1,267,641$ & not considered & $-\$ 243,484$ & $\$ 1,024,157$ & 0.19 \\
\hline Scenario 3 & $\$ 1,267,641$ & $-\$ 4,591,080$ & $-\$ 1,119,334$ & $-\$ 4,442,773$ & 4.50 \\
\hline Scenario 4 & $\$ 1,267,641$ & not considered & $-\$ 1,119,334$ & $\$ 148,307$ & 0.88 \\
\hline
\end{tabular}

Note: a negative cost indicates a saving

However, these costs are still covered by the City of Ottawa's annual RLC budget of approximately $\$ 1$ million, which was discussed earlier in Section 4.5.1.

The benefit-cost ratio equals the annual net benefits divided by the annual RLC costs. The benefit-cost ratio for Scenario 1 is 4.50 , which exceeds 1.0 , meaning that the RLC program's benefits exceed its costs. Hence, the RLC program generated a considerable net benefit for Ottawa. Based on these findings, running the RLC program at the 34 intersections in Ottawa proves to be a benefit socially and economically.

To assess the impacts spread over future years, the net impacts in each year need to be discounted to a present value. Results from Scenario 3 were used since they consider the spillover effects and the benefit from the fine revenues. The economic safety benefits were calculated for the RLC intersections over the 5-year benefit accrual timeframe and converted into NPV. The results are presented in Table 6.3. All costs are in present value terms and are discounted at a rate of $5 \%$ over 5 years.

Results indicate that the estimated $\$ 1.3$ million investment made annually to install, maintain and operate RLCs generates a return of more than three times this amount after one year. The NPV of the benefits is summed for the 5-year service life of the RLC program (until the next contract renewal) yielding an estimated revenue of $\$ 18$ 
Table 6.3 - Annual monetary benefits associated with the RLC treatment for each of the five years of service life

\begin{tabular}{|l|r|r|r|r|r|}
\hline Year & 1 & 2 & 3 & 4 & 5 \\
\hline Discount rate & $5 \%$ & $5 \%$ & $5 \%$ & $5 \%$ & $5 \%$ \\
\hline $\begin{array}{l}\text { Estimated } \\
\text { Benefits }\end{array}$ & $-\$ 5,710,414$ & $-\$ 5,710,414$ & $-\$ 5,710,414$ & $-\$ 5,710,414$ & $-\$ 5,710,414$ \\
\hline Estimated Costs & $\$ 1,267,641$ & $\$ 1,267,641$ & $\$ 1,267,641$ & $\$ 1,267,641$ & $\$ 1,267,641$ \\
\hline $\begin{array}{l}\text { Benefit-Cost } \\
\text { Ratio }\end{array}$ & 4.50 & 4.50 & 4.50 & 4.50 & 4.50 \\
\hline Discount Factor & 0.952 & 0.907 & 0.864 & 0.823 & 0.784 \\
\hline $\begin{array}{l}\text { Discounted } \\
\text { Benefits }\end{array}$ & $-\$ 5,438,489$ & $-\$ 5,179,514$ & $-\$ 4,932,870$ & $-\$ 4,697,972$ & $-\$ 4,474,259$ \\
\hline $\begin{array}{l}\text { NPV (discounted } \\
\text { benefits-costs) }\end{array}$ & $-\$ 4,170,848$ & $-\$ 3,911,873$ & $-\$ 3,665,229$ & $-\$ 3,430,331$ & $-\$ 3,206,618$ \\
\hline $\begin{array}{l}\text { Sum of 5 year } \\
\text { discounted } \\
\text { benefits and } \\
\text { costs= }\end{array}$ & & & & & \\
\hline
\end{tabular}

million. This calculation again proves that the RLC program in Ottawa is a benefit socially and economically in the long term.

\subsection{Sensitivity Analysis}

Various assumptions and estimations were made and used in the base-case analysis of this thesis. These assumptions and estimations introduce potential error and uncertainty to the conclusions. The potential bias is partially addressed by conducting a sensitivity analysis in this section. Ranges of plausible scenarios and assumptions related to the estimated collision reduction frequencies and the social costs were altered to test the robustness of the base-case conclusions. Table 6.4 presents the alternate values considered and provides brief descriptions of the rationales.

Assumptions regarding the collision reduction estimations based on the RLC treatment were tested. Parameter values were selected from probable ranges estimated from the different statistical methods performed in Chapter 5. The estimated social collision costs (from Table 4.9) have not been challenged in the economics literature. The 
Table 6.4 - Alternative values for sensitivity analysis

\begin{tabular}{|c|c|c|c|}
\hline Variable & Base-case & Alternative values & Rationale \\
\hline Expected total collisions & $-2.1 \%$ & $-2.1 \%$ and $5.6 \%$ & \multirow{5}{*}{$\begin{array}{l}\text { Min. and max. estimates obtained } \\
\text { from the different statistical } \\
\text { approaches (Table 5.12) }\end{array}$} \\
\hline $\begin{array}{l}\text { Expected rear-end } \\
\text { collisions }\end{array}$ & $11.9 \%$ & $1.8 \%$ and $23.8 \%$ & \\
\hline $\begin{array}{l}\text { Expected angle } \\
\text { collisions }\end{array}$ & $-18.9 \%$ & $-11.7 \%$ and $-53.8 \%$ & \\
\hline $\begin{array}{l}\text { Expected turning } \\
\text { movement collisions }\end{array}$ & $-20.8 \%$ & $-8.7 \%$ and $-22.2 \%$ & \\
\hline $\begin{array}{l}\text { Expected sideswipe } \\
\text { collisions }\end{array}$ & $6.7 \%$ & $6.7 \%$ and $34.5 \%$ & \\
\hline Value of PDO collision & $\$ 10,000$ & $\begin{array}{l}\$ 9,792^{*} \text { and } \\
\$ 11,000^{* *}\end{array}$ & \multirow{3}{*}{$\begin{array}{l}\text { From: } \\
*(\text { Pitel and Solomon, 2013) - Based } \\
\text { on Canada-wide average social costs } \\
\text { of collisions (calculated from Table } \\
12 \text { and } 13 \\
* *(\text { de Leur, 2010) - Ontario costs } \\
\text { report Table } 7.2 \\
* * *(\text { Vodden } \text { et al., 2007) - Exhibit } \\
\text { III-5 Lower and upper scenarios }\end{array}$} \\
\hline Value of injury collision & $\$ 101,000$ & $\begin{array}{r}\$ 78,781^{*} \text { and } \\
\$ 134,000^{* *}\end{array}$ & \\
\hline Value of fatal collision & $\$ 19,500,000$ & $\begin{array}{c}\$ 9,230,000^{* * *} \text { and } \\
\$ 24,250,000^{* * *}\end{array}$ & \\
\hline
\end{tabular}

base-case for social costs used in the thesis includes the most plausible assumptions gathered from previous studies and represents the outcome that has the highest probability of occurring. Ranges of plausible social cost scenarios are tested for robustness of the base-case conclusions. These tested scenarios can be treated as one of a number of possible values that can be selected in a model which can address the potential for under-reporting and misreporting of collisions. These errors could also have materially affected the conclusion of the study. The sensitivity analysis gives an impression of the dynamics of the situation.

One of the main concerns is that fatalities represent a small proportion of the total collisions, less than 1 percent, yet the comprehensive and human capital cost assigned to a fatality is around 30 times the injury collision costs within the same collision impact type. Thus, one or two fatalities in either the before or after-period for a given intersection could greatly affect the cost-related analysis. While this influence could be 
legitimate if one could expect repeated similar samples to have the same number of fatalities, this is not likely, due to the rare and random occurrence of fatal collisions.

Table 6.5 presents a summary of the sensitivity analysis, and detailed estimations can be found in Appendix L. Results from the sensitivity analysis suggests that the base case analysis results are robust for all plausible alternate assumptions. The resulting net present benefits ranged from $\$ 2.7$ million under the most conservative RLC effect assumptions, to $\$ 3.6$ million based on the highest social costs scenario. For comparison and illustration purposes, the base-case results are also presented in the table as the first column next to the various scenarios. These sensitivity cost estimates could be used for RLC safety program implementation funding or further treatment-development research efforts.

Table 6.5 - Summary of sensitivity analysis

\begin{tabular}{|c|c|c|c|c|c|}
\hline \multirow[b]{2}{*}{ Collision Impact Type } & \multirow[b]{2}{*}{ Base Case } & \multicolumn{2}{|c|}{ Cost } & \multicolumn{2}{|c|}{ RLC Effectiveness } \\
\hline & & $\begin{array}{l}\text { High } \\
\text { Scenario }\end{array}$ & $\begin{array}{c}\text { Low } \\
\text { Scenario }\end{array}$ & $\begin{array}{l}\text { Highest } \\
\text { Reduction } \\
\text { Scenario }\end{array}$ & $\begin{array}{l}\text { Lowest } \\
\text { Reduction } \\
\text { Scenario }\end{array}$ \\
\hline Total collisions & $-\$ 243,484$ & $-\$ 307,367$ & -\$ 170,817 & $-\$ \quad 243,484$ & $\$ 640,701$ \\
\hline Rear-end collisions & $\$ 389,153$ & $\$ 491,875$ & $\$ 322,791$ & 59,262 & \$ 779,771 \\
\hline Angle collisions & $-\$ 1,083,493$ & $-\$ 1,365,322$ & $-\$ 622,712$ & $-\$ 3,089,066$ & $-\$ 671,786$ \\
\hline $\begin{array}{l}\text { Turning movement } \\
\text { collisions }\end{array}$ & $-\$ 295,273$ & $-\$ 378,358$ & $-\$ 240,223$ & $-\$ \quad 315,364$ & $-\$ 123,589$ \\
\hline Sideswipe collisions & $\$ 26,307$ & $\$ 30,926$ & $\$ 23,945$ & $\$ \quad 26,307$ & $\$ 134,664$ \\
\hline Annual RLC costs & $\$ 1,267,641$ & $\$ 1,267,641$ & $\$ 1,267,641$ & $\$ 1,267,641$ & $\$ 1,267,641$ \\
\hline Annual fine revenue & $-\$ 4,591,080$ & $-\$ 4,591,080$ & $-\$ 4,591,080$ & $-\$ 4,591,080$ & $-\$ 4,591,080$ \\
\hline Collision reduction benefit & $-\$ 243,484$ & $-\$ 307,367$ & $-\$ 170,817$ & $-\$ \quad 243,484$ & $\$ 640,701$ \\
\hline NPV (benefit-cost) & $-\$ 3,566,923$ & $-\$ 3,630,806$ & $-\$ 3,494,256$ & $-\$ 3,566,923$ & $-\$ 2,682,738$ \\
\hline Benefit-cost ratio & 3.81 & 3.86 & 3.76 & 3.81 & 3.12 \\
\hline
\end{tabular}




\section{Guidelines, Conclusions and Recommendations}

The first section of this concluding chapter provides planners, managers, administrators, law enforcement, and policy makers with an overview of guiding principles that will support them in the evaluation of the RLC program. Section 7.2 provides a summary of the main findings and Section 7.3 presents recommendations for future research.

\subsection{Guidelines for Practical Implementation}

The following guidelines, formulated based on the results of this thesis, list a selection of relevant tools and sets out a number of key observations so that a RLC program can be tailored to the needs of a particular jurisdiction. These guidelines apply to all municipalities that are currently using the RLC treatment or are planning to use this treatment:

1. Traffic collisions are relatively infrequent events and, if collision statistics are analyzed for a limited number of intersections or for only a short period of time, trends may be missed or misinterpreted. Large reductions in collisions for what appears to be a high risk area may, in fact, be no more than a RTM effect. Using data for a more extended (three to five year) period will be more beneficial so that a meaningful change in the series will likely be detected, thus, ensuring that resources are not inefficiently invested.

2. Program evaluation activities should begin long before RLC is implemented. It is important to get baseline collision data that represent the time before driver behavior was influenced by any RLCs in the area. It is helpful to collect data before the marketing and media campaign begins because the campaign can influence driver behavior. 
3. Since policy decisions are mainly based on the results found from the treatment analysis, it is important to ensure that the statistical tests utilized are not biased. Both NB regression analysis and $\mathrm{EB}$ analysis can lead to misleading results when the covariate distributions in the treatment and control groups are different. Thus, traditional regression models are unable to reconstruct counterfactuals. Matching methods provide the comparison of treated and control groups with similar covariate distributions, to avoid extrapolation in regression models when there is little or no overlap in distributions. This thesis raises concerns about placing heavy reliance on the results of traditional regression methods. Policymakers should be well advised to place less reliance on regression, and to facilitate replication of already conducted studies with a more robust research design and utilize better quality data to avoid missing opportunities for better researched policy.

4. The application of FE and RE panel count modeling techniques should be used to model any non-negative integer count data in a form of panel structure in future research. It measures both cross-sectional and time-series effect, as it did in this thesis, modeling the collisions between intersections as well as over the time.

5. RLCs were found to be more beneficial at intersections with a higher frequency of angle and turning movement collisions to rear-end and sideswipe collisions. Where RLCs are installed, rear-end collisions should be especially closely monitored, since drivers tend to react unpredictably when they notice it's a RLC intersection, leading to a greater risk of rear-end collisions. The high increase in rear-end collisions may negate any benefit realized from the expected decrease in angle collisions, and may be cause for a relocation study of the RLC to an alternative location. Therefore, focusing on target collisions is determined to be more valuable than simply examining total collisions. 
Presenting results related to the total collisions only may not give a clear picture of the effect of the intervention because the increases in rear-end collisions can offset reductions in the main target group of angle collisions.

6. There are a number of practical and theoretical considerations resulting from the interaction terms. It is to be expected that RLC performance will not be the same for all intersections. There may be other traffic and geometrical factors that contribute to a successful RLC application. Such information should be useful for establishing site selection criteria for future RLC locations. It is important to identify the subgroups of intersections in which the treatment is likely to have the largest effect since resources are usually limited to treat only a small fraction of the population. Methods for assessing additive interaction can help determine which subgroups would benefit most from treatment. This thesis presents some potentially valuable implications on program attributes that impact preferences for RLC programs. Given the variation in intersection characteristics, following these guidelines will help increase the likelihood of the RLC program's success for a City similar to Ottawa, ON:

\section{To reduce rear-end collisions:}

$\checkmark$ Installing RLCs at intersections with one dedicated left-turn lanes reduces rearend collisions by an estimated $32 \%$

x Avoid installing RLCs at intersections with right-turn channelization, which were found to increase rear-end collisions by an estimated $39 \%$.

\section{$>$ To reduce angle collisions:}

$\checkmark$ Installing RLCs at intersections with a median reduces angle collisions by an estimated $48 \%$. 
$\checkmark$ Installing RLCs at intersections with right-turn channelization reduces angle collisions by an estimated $46 \%$.

$\checkmark$ Installing RLCs at intersections with protected-permitted left-turn signals reduces angle collisions by an estimated $81 \%$.

$\checkmark$ Installing RLCs at intersections with 4 thru lanes reduces angle collisions by an estimated $82 \%$.

x Avoid installing RLCs at intersections with only 1 dedicated left-turn lane as they increase angle collisions by an estimated $82 \%$.

\section{$>$ To reduce turning movement collisions:}

$\checkmark$ Installing RLCs at intersections with 1 dedicated left-turn lanes reduces turning movement collisions by an estimated $49 \%$.

$\checkmark$ Installing RLCs at intersections with a permitted left-turn signal reduces turning movement collisions by an estimated $75 \%$.

$\checkmark$ Installing RLCs at intersections with 2 thru lanes reduces turning movement collisions by an estimated $51 \%$.

$\checkmark$ Installing RLCs at intersections with $60 \mathrm{~km} / \mathrm{h}$ posted speed limit reduces turning movement collisions by an estimated $68 \%$.

× Avoid installing RLCs at intersections with $70 \mathrm{~km} / \mathrm{h}$ posted speed limit as they increase turning movement collisions by a little over $1 \%$.

\section{To reduce sideswipe collisions:}

$\checkmark$ Installing RLCs at intersections with a median reduces sideswipe collisions by an estimated $77 \%$. 
$\checkmark$ Installing RLCs at intersections with permitted left-turn signals reduces sideswipe collisions by an estimated $43 \%$.

$\checkmark$ Installing RLCs at intersections with 4 thru lanes reduces sideswipe collisions by an estimated $73 \%$.

× Avoid installing RLCs at intersections with right-turn channelization, which were found to increase sideswipe collisions by an estimated $86 \%$.

x Avoid installing RLCs at intersections with 2 thru lanes as they increase sideswipe collisions by an estimated $44 \%$.

These intersection characteristics should be factors to consider when planning for new RLC installations or when studying relocations.

7. Installing a RLC at an intersection where there are other intersections at a very close proximity of under $500 \mathrm{~m}$ increases turning movement collisions by an estimated $70 \%$, but reduces the rear-end collisions by $60 \%$. Having a RLC intersection within 500 $\mathrm{m}$ to $1 \mathrm{~km}$ away from a specific intersection reduced rear-end collisions by $15 \%$, but increases turning movement and sideswipe collisions by an estimated $48 \%$ and $50 \%$, respectively. It is also important to consider the geographic distribution of the RLC sites, as having several RLC sites in close proximity to each other can possibly reduce their effectiveness. Having more RLCs condensed within a zone of under $500 \mathrm{~m}$ would have a massive impact in increasing the rear-end collisions and not having any significant effect in reducing angle collisions. Having two or more RLCs within a $500 \mathrm{~m}$ to a $1 \mathrm{~km}$ distance seems to have better results on collision reductions, where there is an estimated decrease in angle collisions by $77 \%$, a reduction in turning movement collisions by $61 \%$ and a reduction in sideswipe collisions by $92 \%$. There should be a balanced use of RLCs 
throughout a jurisdiction. In many cases, unveiling the mechanisms behind the spillover effects will lead to a better understanding of how the program works in general.

8. Relocation consideration of RLCs should be considered if evaluation assessments prove that they no longer serve their original purpose. These evaluations should be based on collision reductions and social cost savings. It would be useful to regularly review what is happening at individual locations, and not overall totals of all intersections combined for a city, in order to determine the on-going suitability and need for RLCs at each specific intersection. This review should include an examination of the level of RLR violations and collisions at each intersection, any changes to street furniture, road traffic or construction and other relevant considerations. Such a periodic review will provide additional insight into program operations and may serve to identify appropriate modifications to the program. This analysis could lead management to rotate RLCs rather than installing new RLCs at other high-frequency collision intersections. RLC installment and relocation decisions represent a major policy action, with serious repercussions for public safety.

The guidelines in this section provide direction on the development of policies for traffic safety outcomes. This includes the need for ongoing evaluations and the development of a traffic safety plan that includes appropriate interventions and proper impact assessments methods to base the policy decisions. The development of RLC policy follows the same process, and should be considered as part of broader road safety policy development. These set of guidelines aim to assist in this key policy effort by enhancing such decisions using rigorous research and novel methods. 


\subsection{Conclusions}

Road collisions place a great burden on individuals, property and society. Research shows that many drivers routinely violate the red-light signals, placing themselves and other road users at risk for serious collisions. One of the key enforcement initiatives to assist and facilitate enforcement against RLR is RLCs. The methodologies, population sizes, and data sources used to assess effectiveness of RLC programs have varied, which may lead to flawed collision frequency predictions and incorrect inferences relating to the factors that determine the frequency of collisions. At the present time, there is a lack of clear guidelines to use when evaluating the decision to install RLCs at specific intersections.

Contributions of this thesis to the literature include: (1) correcting for the siteselection bias of traditional methodologies by using a novel methodology (propensity score matching); (2) utilizing the fixed-effect and random-effect panel regressions to account for the spatial and temporal correlations in the data; (3) identifying how RLC effectiveness varies by site characteristics by using interaction terms; (4) measuring spillover distances and times resulting from the presence of RLCs; (5) applying benefitcost methodology supported by economic analysis and sensitivity analysis using these new estimation methods; and (6) establishing guidelines for effective implementation of the RLC treatment. Data from signalized intersections in the City of Ottawa were used to conduct the analyses.

This thesis first explored the suitability of traditional observational models (i.e. variations of the NB and the EB methods). Statistical tests such as the Vuong test and the AIC, were calculated to identify the best fit models. Based on the final model estimates 
and incident rate ratios, the safety effects of the RLCs and other control variables were evaluated. Next, a comparison of results from different safety evaluation methods was conducted to establish a framework for assessing and evaluating the regional conditions necessary to make proper decision on using RLCs. The results of EB collision-frequency analyses at the treated intersections indicate that RLCs have effects similar in direction but somewhat smaller in magnitude than those indicated in the NB regression. The PSM method was introduced in attempt to create equivalent treatment and control groups by summarizing relevant pre-treatment characteristics of each subject into a single-index variable, the propensity score. Then, matching subjects in the untreated comparison pool to subjects in the treatment group based on values of the propensity score.

While the results varied a bit across the different methods, the direction and magnitude were remarkably consistent. The results found that the RLC program in the City of Ottawa was effective overall with a total collision reduction of $10 \%$, with an estimated net economic benefit of approximately $\$ 4.4$ million per year (including spillover effects and fine revenues). The collision reduction benefits include the avoided collision costs associated with a $19 \%$ reduction in angle collisions (savings $>\$ 1,300,000$ ), a $21 \%$ reduction in turning movement collisions (savings $>\$ 295,000$ ) plus the revenue from issued citations. However, the RLC presence is estimated to increase the rear-end collision frequencies by $25 \%$ (a loss $>\$ 710,000)$ and sideswipe collisions by $7 \%$ (a loss $>\$ 26,000)$. The final benefit-cost ratio which includes the total annual collision reduction benefits, the spillover effects and annual fine revenue is 4.50. Based on these findings, running the RLC program at the 34 intersections in Ottawa proves to be a benefit socially 
and economically. These results are robust to all plausible scenarios tested in the sensitivity analysis.

The main strength of this thesis, which sets it apart from previous research, lies in the development of a procedure for estimating more accurately the actual benefits of RLC deployment by adjusting covariates between the treated and control groups. The PSM method allows scholars to reconstruct counterfactuals using observational data. Misspecified models using observational data sometimes produce biased estimators. One source of such bias is that the two samples lack distribution overlap, and regression analysis cannot tell researchers the distribution overlap between two samples. PSM should be used, at least as a robust test to justify the findings estimated by regression models.

This thesis has presented evidence to suggest that conventional approaches to account for RTM effects that rely on regression techniques could lead to over/under optimistic assessments of the value of road safety measures, suggesting that benefit-cost decisions may not be optimal. It is recommended here that a PSM approach is adopted which is statistically more appropriate to handling casualty data and flexible enough to allow confounding factors to be incorporated more rigorously with the end result of more reliable estimates of the impacts of road safety measures.

This thesis has also demonstrated the value of including the interaction effects to provide site selection criteria for future RLC site selection. Site selection issues are extremely important, where the aim is to generate maximum return in terms of casualty reduction. It would be very impulsive to conclude that RLCs are uniformly effective in reducing traffic collisions, as did many other studies. Hence a treatment decision based 
on the average treatment effect for the entire population can be misleading and should be dependent on site characteristics. Since RLCs are usually costly, it is desirable that the RLC program is operated in a way that maximizes effectiveness with limited resources. By revealing patterns of the RLC treatment effects, and any other traffic and geometrical factors that contribute to a successful RLC application, policy makers can install cameras at sites most likely to benefit from the treatment to improve the cost-effectiveness of the program.

The safety benefits extend beyond the immediate vicinity of the RLC intersections, evidenced by a significant spillover effect in the reduction of angle and turning movement collisions in the zones where RLCs are present. Recognizing that collisions are the result of complex interactions amongst many factors, and subject to considerable randomness, these deviations should be used as opportunities for detailed investigation and learning to design and deploy more effective automated enforcement programs. The thesis confirms the importance of implementing RLCs on a case-by-case basis only after a careful review of the collision patterns and geometric/operational characteristics at each intersection. The procedure developed as part of the thesis also helps to identify intersections, presently without enforcement, that are likely to benefit from the RLC treatment.

In conclusion, the results obtained in this thesis provide an important step forward toward optimizing a systematic, statistically defensible approach to identifying the impacts of the RLC. A series of issues related to RLCs addressed in this research have not been investigated in depth before, such as how effectiveness varies by site characteristics and under what conditions RLCs perform most effectively. This thesis 
contributes to the literature by applying novel causal methods and utilizing spatial analysis to estimate treatment effects of RLCs on traffic safety to help set guidelines for practical implementation.

\subsection{Recommendations for Future Research}

This thesis addressed some of the gaps in the literature on the casual link between the RLC treatment and road safety. In this section, further recommendations for future research efforts are suggested below:

- This thesis, as well as all reports in Ontario assessing social costs, was based on data outlining the social cost figures from the year 2004 for different collision classifications. There is a considerable need to develop new and updated social cost estimates to be used in all treatment effect analysis as part of a larger effort of producing more accurate cost estimates.

- Additional variables that may influence intersection safety need to be investigated when running causal models. Some variable suggestions that would be useful to consider in future studies include: pavement conditions, visibility of road markings, sizes of the LED red lights on the traffic signals, seatbelt use, and drunk/impaired driver status.

- An analysis similar to the one performed in this thesis should be conducted on a larger number of intersections (perhaps the entire collision database of all intersections in the city). Such study would allow a further, more accurate exploration of the determinants of intersection-collision frequencies.

- Impacts of signal timing need to be studied further to determine how signal timing parameters, particularly cycle length and coordination, influence the safety effects 
of RLCs and traffic patterns in the field. Knowledge on the effects of cycle length is limited. Cycle length is needed to provide some measure of the number of red phases (and thus the number of opportunities for RLR) in a given time period. Also, longer red phases might induce more RLR. The length of the phasing during the AM and PM peak should also be reviewed. With respect to signal coordination, a review of whether or not the treated signal approach is part of a set of coordinated signals that lead to queuing of vehicles. Poorly coordinated traffic signals may lead to driver frustration and in turn RLR at signalized intersections.

- A finding in the literature requires further investigation from Huey and Ragland (2007) and Nambisan and Karkee (2010). The finding states that intersections with signal progression, typically provided for pedestrians, where through vehicles can see the countdown, have a lower likelihood of rear-end collisions. Such progression decreases the amount of stops and starts vehicles have to make, which is a decreasing rear-end collision potential. In future studies, the possibility of using progression on all intersection approaches that are in close proximity to other signalized intersections should be explored. Data in this thesis did not cover any signal-progression information. Such information would be valuable when analyzing all intersections in the city. 


\section{References}

Alberta Justice and Solicitor General. (2014). Automated Traffic Enforcement Technology Guidelines. Province of Alberta. Available at: https://www.solgps.alberta.ca/programs_and_services/public_security/law_enforcement_ oversight/Publications/ATE\%20Technology\%20Guidelines\%20Guidelines\%20(Sept\%20 2014).pdf Accessed on: Jul. 10, 2017.

AASHTO. (2010). Highway Safety Manual. American Association of State Highway and Transportation Officials. Washington, D.C. Available at: http://www.highwaysafetymanual.org Accessed on: Jul. 10, 2017.

AECOM. (2014). Intersection safety device program - Red-light camera analysis. Alberta Transportation. ON, Canada.

Aeron-Thomas, A., \& Hess, S. (2005). Red-light cameras for the prevention of road traffic collisions (Review). Oxfordshire, England: The Cochrane Collaboration, Available at: http://www.thecochranelibrary.com/userfiles/ccoch/file/Safety_on_the_road/CD003862.p df Accessed on: Jul. 10, 2017.

Allison, P. (2012). Logistic regression using SAS: Theory and application. (2nd Ed.) Cary, NC: SAS Institute.

Allison, P. (2015). Getting the Lags Right. Statistical Horizons. Available at: http://statisticalhorizons.com/getting-the-lags-right Accessed on: Jul. 10, 2017.

Andreassen, D. (1995). A Long Term Study of Red Light Cameras and Accidents. Research Report \#261. Australian Road Research Board. Victoria, Australia.

Austin, P.C. (2009). Balance Diagnostics for Comparing the Distribution of Baseline Covariates between Treatment Groups in Propensity-Score Matched Samples. Statistics in Medicine, 28: pp.3083-3107.

Austin, P.C. (2011). A Tutorial and Case Study in Propensity Score Analysis: An Application to Estimating the Effect of In-Hospital Smoking Cessation Counseling on Mortality. Multivariate Behavioral Research. 46:119-151.

Bissonette, J. A. and Cramer, P. C. (2008). Evaluation of the use and effectiveness of wildlife crossings. NCHRP Report 615. National Cooperative Highway Research Program, Transportation Research Board, National Academies, Washington, D.C.

Bördős, K., Csillag, M. and Scharle, Á. (2015) The effect of children's disability on the labour supply of mothers in Hungary. Budapest Institute for Policy Analysis. 9th annual conference of the Hungarian Society of Economics. Available at: http://www.budapestinstitute.eu/uploads/impact_childrens_disability_maternal_employm ent.pdf Accessed on: Jul. 10, 2017.

Boris, C., Carlos, S. (2016). Safety effectiveness and crash cost benefit of red light cameras in Missouri. Traffic Injury Prevention, 18(1): pp 70-76.

Boudreaux, C. (2014). An Empirical Analysis of Red Light Traffic Cameras: Do They Increase Public Safety or Is There a Revenue Motive? Job Market Paper. Available at: http://myweb.fsu.edu/cjb09f/RLCjobmarketpaper.pdf Accessed on: Jul. 10, 2017.

Brault, M., Auger, A., Montégiani, M. (2007). La sécurité aux intersections : analyse des comportements des conducteurs au feu rouge. Présentation à la XVIIe Conférence Canadienne Multidisciplinaire sur la Sécurité Routière à Montréal, 3-6 juin 2007. 
Brookhart, M.A., Schneeweiss, S., Rothman, K.J., Glynn, R.J., Avorn, J., Stürmer, T. (2006). Variable selection for propensity score models. American Journal of Epidemiology, 163(12):1149-1156.

Budd, L., Scully, J. \& Newstead, S. (2011) Evaluation of the Collision Effects of Victoria's Fixed Digital Speed and Red-Light Cameras, Monash University Collision Research Centre.

Buis, M.L. (2010). StataSE tip 87: Interpretation of interactions in nonlinear models. The StataSE Journal. Number 2, pp. 305-308. Available at: http://www.StataSE journal.com/sjpdf.html?articlenum=st0194 Accessed on: Jul. 10, 2017.

Burkey, M. and K. Obeng, A. (2004). Detailed Investigation of Collision Risk Reduction Resulting from Red Light Cameras in Small Urban Areas. Report for USDOT, 60 pp. Urban Transit Institute, the Transportation Institute, North Carolina Agricultural and Technical State University, Greensboro.

Calgary Police Service. (2013). Speed on green and red light cameras. Available at: http://www.calgary.ca/cps/Pages/Traffic/Speed-on-green-and-red-light-cameras.aspx

Caliendo, M., and Kopeinig, S. (2005). Some practical guidance for the implementation of propensity score matching. IZA Discussion Paper No. 1588. Bonn, Germany. Available at: http://ftp.iza.org/dp1588.pdf. Accessed on: Jul. 10, 2017.

Champness, P., and Folkman, L. (2005). Time and distance halo effects of an overtly deployed mobile speed camera. The Australasian Road Safety Research, Policing and Education Conference Proceedings.

Chen, H., Meuleners, L., Hendrie, D. (2012). The effectiveness and cost-effectiveness of upgraded red light speed cameras in Western Australia: a preliminary analysis. CurtinMonash Accident Research Centre. Available at: http://cmarc.curtin.edu.au/local/docs/RLScamera_Report_May2012.pdf Accessed on: Jul. 10, 2017.

Chen, F., Chen, S., \& Ma, X. (2016). Crash Frequency Modeling Using Real-Time Environmental and Traffic Data and Unbalanced Panel Data Models. International Journal of Environmental Research and Public Health, 13(6), 609. http://doi.org/10.3390/ijerph13060609 Accessed on: Jul. 10, 2017.

Christie, S. M. Lyons, R. A., Dunstan, F. D., and Jones, S. J. (2003). Are mobile speed cameras effective? A controlled before and after study. Injury Prevention, 9(4), pp. 302306.

City of Ottawa. (2012). Ottawa's Strategic Road Safety Action Plan. Available at: http://ttawa.ca/calendar/ottawa/citycouncil/occ/2011/12-14/cpsc/02\%20-

\%20Safe\%20Roads\%20Document\%201\%20-\%20Action\%20Plan\%20-\%20English.pdf

Accessed on: Jul. 10, 2017.

City of Ottawa. (2016). Red-light cameras. Ottawa, Ontario. Available at: http://ottawa.ca/en/residents/transportation-and-parking/road-safety/red-light-cameras Accessed on: Jul. 10, 2017.

City of Ottawa. (2017). Annual Safety Reports. Available at: http://ottawa.ca/en/residents/transportation-and-parking/road-safety/annual-safetyreports. Accessed on: Jul. 10, 2017.

City of Saskatoon. (2014). Red Light Cameras. Available at: https://www.saskatoon.ca/moving-around/driving-roadways/safe-driving/red-lightcameras Accessed on: Jul. 10, 2017. 
City of Toronto. (2017). Red Light Cameras get extended stay on our roads. Road Safety. Available at: http://www1.toronto.ca/wps/portal/contentonly?vgnextoid=17324074781e1410 VgnVCM 10000071d60f89RCRD Accessed on: Jul. 10, 2017.

Cohan, S. (2017). Electronic Red Light Safety Program. Program Report for CY 2016. Delaware Department of Transportation. Available at: http://deldot.gov/information/red_light/pdfs/after_analyses_summary2016.pdf Accessed on: Jul. 10, 2017.

Council, F.M., B. Persaud, K. Ecces, C. Lyon, and M. S. Griffith. (2005). Safety evaluation of red-light cameras. No. FHWA-HRT-05-048. FHWA, U.S. Department of Transportation. Available at: https://www.fhwa.dot.gov/publications/research/safety/05048/05048.pdf Accessed on: Jul. 10, 2017.

CTV News. (2015). More than 17,000 cars caught by red light cameras in 2014. Available at: http://ottawa.ctvnews.ca/more-than-17-000-cars-caught-by-red-light-cameras-in-20141.2416091 Accessed on: Jul. 10, 2017.

Currie and Basak. (2015). Red Light Cameras - Status Update and Phase 3 Contract Award (Request for Proposal No. 9148-15-5000 - 2017 to 2021). Staff Report. Available at: http://www.toronto.ca/legdocs/mmis/2015/pw/bgrd/backgroundfile-83498.pdf Accessed on: Jul. 10, 2017.

D'Agostino, R.B. (2007). Propensity Scores in Cardiovascular Research. Statistical Primer for Cardiovascular Avearch. Available https://doi.org/10.1161/CIRCULATIONAHA.105.594952 Accessed on: Jul. 10, 2017.

Data Analysis and Statistical Software. (2015). Obtaining marginal effects after estimations with interactions. Stata Available at: http://www.stata.com/support/faqs/statistics/marginal-effects-methods/ Accessed on: Jul. $10,2017$.

Date, J. (2014). Memorandum. IBI Group. Available at: http://webcast.ottawa.ca/plan/All_Image\%20Referencing_Subdivision_Image\%20Refere nce_D07-16-13-0021\%20Transportation\%20Study.PDF Accessed on: Jul. 10, 2017.

Dawson, J. (2015). Interpreting interaction effects. The University of Sheffield. Available at: http://www.jeremydawson.com/slopes.htm Accessed on: Jul. 10, 2017

De Leur, P. (2010). Collision Cost Study. Capital Region Intersection Safety Partnership. Available at: http://drivetolive.ca/wpcontent/uploads/2014/02/Collision_Cost_Study_Final_Report_Feb_2010.pdf Accessed on: Jul. 10, 2017.

De Leur, P. (2011). Site selection process and methodology for the deployment of intersection safety cameras in British Columbia. Transportation Research Record Number 2011, Journal of the Transportation Research Board, pp. 129-136.

De Pauw, E., Daniels, S., Brijs, T., Hermans, E., \& Wets, G. (2014). To brake or to accelerate? Safety effects of combined speed and red light cameras. Journal of Safety Research, 50: pp. 59-65.

Dodge, B. (2016). "New red light camera installed at Old Tenth Line and St. Joseph." Ottawa West News. Available at: https://www.ottawacommunitynews.com/news-story/6892088new-red-light-camera-installed-at-old-tenth-line-and-st-joseph/ Accessed on: Jul. 10, 2017. 
Donnell, E., Shankar, V., and Richard, P. (2009). Analysis of Safety Effects for the Presence of Roadway Lighting. University Park, PA: The Pennsylvania State University. Available at http://onlinepubs.trb.org/onlinepubs/nchrp/docs/NCHRP05-19_SafetyReport.pdf Accessed on: Jul. 10, 2017.

Drichoutis, A. (2011). Interpreting interaction terms in linear and non-linear models: A cautionary tale. Department of Economics, University of Ioannina. MPRA Paper No. 33251, posted 9. Available at: https://mpra.ub.unimuenchen.de/33251/1/MPRA_paper_33251.pdf Accessed on: Jul. 10, 2017.

Eisenberg, D. (2004). The Mixed Effects of Precipitation on Traffic Crashes. Accident Analysis and Prevention. 36: pp.637-647.

Elliott, M., and Broughton, J. (2005). How methods and levels of policing affect road casualty rates (TRL Report 637). Wokingham: Transport Research Laboratory.

Elliott, M., Durbin D., and Flaura K. Winston. (2009). Propensity score approach to estimating child restraint effectiveness in preventing mortality. Statistics and Its Interface. 2: 437447. Available

at: http://intlpress.com/site/pub/files/_fulltext/journals/sii/2009/0002/0004/SII-2009-00020004-a005.pdf Accessed on: Jul. 15, 2017.

Erke, A. (2009). "Red-Light Cameras - A Meta-analysis of the Effects of Red-light Cameras on Collisions", Accident Analysis and Prevention, 41 (5): pp. 897-905.

Federal Highway Administration (FHWA) (2009). Engineering Countermeasures to Reduce Red-Light Running. Available at: http://safety.fhwa.dot.gov/intersection/resources/fhwasa10005/docs/brief_6.pdf Accessed on: Jul. 10, 2017.

Federal Highway Administration FHWA (2010). A Guide to Developing Quality Collision Modification Factors. Available at: http://safety.fhwa.dot.gov/tools/crf/resources/fhwasa10032/ Accessed on: Jul. 12, 2017.

Frost, J. (2015). The Danger of Overfitting Regression Models. The Minitab Blog. Available at: $\quad$ http://blog.minitab.com/blog/adventures-in-statistics-2/the-danger-of-overfittingregression-models Accessed on: Jul. 12, 2017.

Foote, A. (2016). 20 new red light cameras coming to Ottawa by end of 2017 CBC News. Posted: Oct 03, 2016 12:08 PM ET. http://www.cbc.ca/news/canada/ottawa/ottawa-newred-light-cameras-1.3788964 Accessed on: Jul. 10, 2017.

Garber, N.J., Miller, J.S., Abel, R.E., Eslambolchi, S., and Korukonda, S.K. (2007). The impact of red light cameras (photo-red enforcement) on collisions in Virginia. Report no. VTRC 07-R2. Charlottesville, VA: Virginia Transportation Research Council.

Garrido M., Kelley A., Paris J., Roza, K., Meier, D., and Morrison, R. (2014). Methods for constructing and assessing propensity scores. Health Services Research. 49:1701-1720. Available at: https://www.ncbi.nlm.nih.gov/pmc/articles/PMC4213057/ Accessed on: Jul. 10, 2017.

Guo, S., and Fraser, M. (2010). Propensity score analysis: Statistical Methods and Applications. Los Angeles, CA: Sage Publications.

Guerin, P. (2010). City of Albuquerque Red Light Camera Study Final Report. Institute for Social Research - The University of New Mexico. Available at: http://isr.unm.edu/reports/2010/city-of-albuquerque-red-light-camera-study-final-report.Accessed on: Jul. 10, 2017. 
Hagen, L. (2014). Empirical Bayes Analysis For Safety. Traffic Safety Academy. Florida Department of Transportation. Available at: http://www.tampabaytrafficsafety.com/LATSA/LASTA\%20II\%202014\%20Presentations /FDOT\%20D7\%20LATSA\%20II\%20S08\%20Empirical\%20Bayes\%20Analysis\%20for \%20Safety\%20-\%20Hagen.pdf Accessed on: Jul. 10, 2017.

Hall, J.W. (1998). Economic benefit of accident reductions. Proceedings of the 68th Annual Meeting of the Institute of Transportation Engineers. Washington, DC: ITE.

Hanley, P. (2004). Using Crash Costs in Safety Analysis. Public Policy Center. Iowa City, IA: University of Iowa.

Harder, V.S., Stuart, E.A., and Anthony, J. (2010). Propensity Score Techniques and the Assessment of Measured Covariate Balance to Test Causal Associations in Psychological Research. Psychological Methods, 15(3): pp. 234-249.

Hauer, E., (1997) Observational Before-After Studies in Road Safety: Estimating the Effect of Highway and Traffic Engineering Measures on Road Safety. Pergamon Press, Elsevier Science Ltd., Oxford, United Kingdom.

Hauer, E., Harwood, D.W. Council, F.M. and Griffith, M.S. (2001). The Empirical Bayes method for estimating safety: A tutorial. Transportation Research Record 1784, pp. 126131. National Academies Press, Washington, D.C. Available at: http://conferences.ict.illinois.edu/spfsummit09/SPF\%20Basic\%20Tutorial\%202001\%20b y\%20Ezra\%20Hauer.pdf Accessed on: Jul. 10, 2017.

Hausman, J. A., Hall, B. H. and Griliches, Z. (1984). Econometric models for count data with an application to the patents-R\&D relationship. Econometrica, 52(4): pp. 909-938.

Heinrich, C., Maffioli, A. and Vázquez, G. (2010). A Primer for Applying Propensity-Score Matching. Inter-American Development Bank Impact-Evaluation Guidelines Technical Notes No. IDB-TN-161.

Hess, S. (2004). Analysis of the effects of speed limit enforcement cameras: Differentiation by road type and catchment area. Transportation Research Record, 1865: pp. 28-34.

Higgins, J.P., Thompson, S.G., Deeks J.J. and Altman, D.G. (2003). Measuring inconsistency in meta-analyses. $B M J, 327(7414)$ : pp. 557-560. Available at: https://www.ncbi.nlm.nih.gov/pmc/articles/PMC192859/ Accessed on: Jul. 12, 2017.

Hilbe, J. (2008). Brief Overview on Interpreting count Model Risk Ratios - An Addendum to Negative Binomial Regression. Cambridge University Press. http://courses.statistics.com/count/HILBE_NBR_OVERVIEW_ON_INTERPRETING_R ISK_RATIOS.pdf Accessed on: Jul. 12, 2017.

Hillier, W., Ronczka, J. and Schnerring, F. (1993). An Evaluation of Red Light Cameras in Sydney, Research Note RN 1/93, Road Safety Bureau, Roads and Traffic Authority, New South Wales, Australia.

Høye, A. (2013) Still red light for red light cameras? An update. Accident Analysis and Prevention, 55: pp. 77-89.

Hu, W., McCartt, A.T. and Teoh, E.R. (2012). Effects of red light camera enforcement on fatal crashes in large US cities. Journal of Safety Research 42, pp. 277-282.

Huber, C. (2015). Introduction to treatment effects in Stata: Part 1. The STATA Blog. Available at: http://blog.stata.com/2015/07/07/introduction-to-treatment-effects-in-statapart-1/ Accessed on: Jul. 10, 2017. 
Huey, S. B. and Ragland, D. (2007). "Changes in Driver Behavior Resulting from Pedestrian Countdown Signals." In Proceedings of the Transportation Research Board 86th Annual Meeting, Washington, DC: Transportation Research Board, National Research Council.

Imai, K., and M. Ratkovic. (2014). Covariate balancing propensity score. Journal of the Royal Statistical Society, Series B 76: 243-263.

Institute for Digital Research and Education (IDRE). (2001). Regression Models for Count Data. UCLA. Available at: https://stats.idre.ucla.edu/stata/seminars/regression-modelswith-count-data/ Accessed on: Jul. 10, 2017.

Institute for Digital Research and Education (IDRE). (2017). StataSE Class Notes: Analyzin Data. UCLA. Available at: http://statistics.ats.ucla.edu/stat/StataSE /notes/analyze13.htm Accessed on: Jul. 10, 2017.

Insurance Institute for Highway Safety (IIHS) (2013a). Effects of Red Light Camera Enforcement on Red Light Violations in Arlington County, VA.

Insurance Institute for Highway Safety (IIHS). (2013b) Q\&A: Red Light Cameras. Arlington, VA. Available at: http://www.iihs.org/research/qanda/rlr.html. Accessed on: Jul. 8, 2017.

Johansson, P. (1996). Speed limitation and motorway casualties: A time series count data regression approach. Accident Analysis and Prevention, 28(1): pp. 73-87.

Joseph, L. (2016). Interactions in Multiple Linear Regression. Department of Epidemiology, McGill University. Available at: http://www.medicine.mcgill.ca/epidemiology/Joseph/courses/EPIB-621/interaction.pdf Accessed on: Jul. 8, 2017.

Karlaftis, M. and Tarko, A. (1998). Heterogeneity Considerations in Accident Modeling. Accident Analysis and Prevention, 30(4): pp. 425-433.

Keech, J. (2016). Agreements Required For Red Light Camera Program. Report to Council. City of Kingston. Report 16-096. https://www.cityofkingston.ca/documents/10180/13485581/COU_A121616096.pdf/a63b3477-2553-42d4-be80-419ca79a15f9 Accessed on: Jul. 10, 2017.

Keefe, L. (2012). Red Light Camera/Roadside Speed Indicator Update. City of Ottawa. Ref. No. ACS2012-COS-PWS-0012. Available at: http://ottawa.ca/calendar/ottawa/citycouncil/trc/2012/07-04/IPD\%20A\%20-

\%20ACS2012-COS-PWS-0012\%20Red\%20Light\%20Camera.pdf Accessed on: Jul. 8, 2017.

Kloeden, C., Edwards, S. and McLean, A. (2009). Evaluation of South Australian red light and speed cameras. Centre for Automotive Safety Research. Available at: http://casr.adelaide.edu.au/publications/researchreports/casr011.pdf Accessed on: Jul. 8, 2017.

Ko, M., Geedipally, S. R. and Walden, T. D. (2013). Effectiveness and Site Selection Criteria for Red Light Camera Systems. Transportation Research Record: Journal of the Transportation Research Board, No. 2327, Transportation Research Board of the National Academics, Washington, D.C., pp. 53-60.

Kowdla, S. (2004). Modeling collision frequencies at signalized intersections in central Florida. University of Central Florida, Orlando, Florida.

Kyrychenko, S. and Retting, R. (2004). Review of - A detailed investigation of collision risk reduction resulting from red-light cameras in small urban areas - by M. Burkey and K. Obeng. IIHS, November. 
Leclair, R. (2004). Red-light Camera Program - Status Update. Public Works and Services. City of Ottawa. Available at: http://ottawa.ca/calendar/ottawa/citycouncil/trc/2004/1201/ACS2004-TUP-TRF-0005.htm Accessed on: Jul. 10, 2017.

Leuven, E. and Sianesi, B. (2003). "PSMATCH2: Stata Module to Perform Full Mahalanobis and Propensity Score Matching, Common Support Graphing, and Covariate Imbalance Testing, version 4.0.6" Available at http://ideas.repec.org/c/boc/bocode/s432001.html. Accessed on: Jul. 10, 2017.

Lord, D., Washington, S.P. and Ivan, J.N. (2005). Poisson, Poisson-Gamma and Zero-Inflated Regression Models of Motor Vehicle Collisions: Balancing Statistical Fit and Theory. Accident Analysis and Prevention, 37, 35-46. http://dx.doi.org/10.1016/j.aap.2004.02.004 Accessed on: Jul. 7, 2017.

Lord, D. and Mannering, F. (2010). The Statistical Analysis of Crash-Frequency Data: A Review and Assessment of Methodological Alternatives. Transportation Research - Part A; 44(5): pp. 291-305.

Lord, D., and Geedipally, S.R.. (2014). Safety Effects of the Red-Light Camera Enforcement Program in Chicago, Illinois. Research report prepared for the Chicago Tribune, College Station, TX.

Mahmassani, H., Schofer, J., Johnson, B., Verbas, O., Elfar, A., Mittal, A. and Ostojic, M. (2017). Chicago Red Light Camera Enforcement. North Western University Transportation Center. Available at: http://www.transportation.northwestern.edu/docs/research/RLC-Report-Web.pdf Accessed on: Jul. 7, 2017.

Malone, B., Hadayeghi. A., and White. C. (2010). Red-Light Cameras: Surprising New Safety Riesults", ITE Annual Meeting.

Mannering, F. and Bhat, C. (2014). Analytic methods in accident research: Methodological frontier and future directions. Analytic Methods in Accident Research. 1:1-22. Available at: http://cee.eng.usf.edu/faculty/flm/CE615_files/Mannering-Bhat- Accessed on: Jul. $10,2017$.

Metropolitan Knowledge International (MKI). (2011). Ottawa Light Rail Transit (OLRT) Updated Business Case - Project Benefits Analysis. http://www.ligneconfederationline.ca/media/pdf/OLRTBusinessCaseUpdate.pdf Accessed on: Jul. 7, 2017.

Ministry of Transportation Ontario (2007). Analysis and Estimation of the Social Cost of Motor Vehicle Collisions in Ontario. Available at: http://www.tc.gc.ca/media/documents/roadsafety/ TP14800E.pdf Accessed on: Jul. 7, 2017.

Ministry of Transportation Ontario (2011). Ontario Road Safety Annual Report 2011. Toronto: Ministry of Transportation Ontario.

Minitab Statistical Software. (2017). Why should I use a 2-sample t test? State College, PA. Available at: http://support.minitab.com/en-us/minitab/17/topic-library/basic-statisticsand-graphs/hypothesis-tests/tests-of-means/why-use-2-sample-t/ Accessed on: Jul. 10, 2017.

Miaou, S., Song, J., Mallick, B. (2003). Roadway traffic crash mapping: A spacetime modeling approach. Journal of Transportation and Statistics. 6(1): pp. 33-57.

Mountain, L. and Fawaz, B. (1992). Effects of engineering measures on safety at adjacent sites. Traffic Engineering Control. 33(1): 15-17. 
Newstead, S.V. \& Cameron, M.H. (2012). Development of an evaluation framework for the Queensland Camera Detected Offence Program (CDOP). Monash University Collision Research Center. Victoria, Australia. ISSN 1835-4815 (online).

Nambisan, S. S., and Karkee, G. J. (2010), "Do Pedestrian Countdown Signals Influence Vehicle Speeds?" Transportation Research Record: Journal of the Transportation Research Board. 2149, Transportation Research Board of the National Academies, Washington, D.C., pp. 70-76.

Noble, J. (2014). COMP6053 lecture: Transformations, polynomial fitting, and interaction terms. University of Southampton, United Kingdom. Available at: http://users.ecs.soton.ac.uk/jn2/teaching/interactions.pdf Accessed on: Jul. 8, 2017.

Noland, R.B. (2003). Traffic fatalities and injuries: the effect of changes in infrastructure and other trends. Accident Analysis and Prevention, 35(4): pp. 599-611.

Obeng, K. and Burkley, M. (2008)."Explaining collisions at intersections with red-light cameras: A note", Transportation Research Part A, 42: pp. 811-817.

Ontario's Regulatory Registry. (2015). Increasing the Reporting Threshold for Property Damage Only (PDO) Collisions in Ontario. Available at: http://www.ontariocanada.com/registry/view.do?postingId=18102 Accessed on: Jul. 8, 2017.

Ottawa Police Service. (2008). Financial Costs Associated with Traffic Collisions. Available at: http://ottawa.ca/calendar/ottawa/citycouncil/opsb/2008/05-26/item8.htm Accessed on: Jul. 8, 2017.

Park, H.M. (2011). Regression Models for Panel Data Using Stata: 8. International University of Japan. Public Management \& Policy Analysis Program. Available at: https://www.google.ca/url?sa=t\&rct=j\&q=\&esrc=s\&source=web\&cd=2\&cad=rja\&uact $=$ 8\&ved=0ahUKEwjLoI261OPTAhUkzIMKHby9BwwQFgg7MAE\&url=https\%3A\%2F\% 2Fwww.researchgate.net\%2Ffile.PostFileLoader.html\%3Fid\%3D5693bd9c7c192090dc8 b4595\%26assetKey\%3DAS\%253A316718184894466\%25401452522907629\&usg=AFQ jCNHV2H1ozuGTW1mVLBjSPibPERBCNw\&sig2=VAuiBsalzPyQmhO2YtYMHw Accessed on: Jul. 12, 2017.

Pearl, J. (2010). The foundations of causal inference. Sociological Methodology. 40(1):75-149.

Pitel, S. and Solomon, R. (2013). Estimating the Number and Cost of Impairment-related Traffic Crashes in Canada: 1999-2010. Mothers Against Drunk Driving (MADD Canada) Available at: http://www.madd.ca/media/docs/estimating_presence.pdf Accessed on: Jul. 9, 2017.

Princeton University Library. (2017). Panel Data. Data and Statistical Services. Available at: http://dss.princeton.edu/online_help/stats_packages/stata/panel.htm Accessed on: Jul. 10, 2017.

Region of Waterloo. (2010). Red Light Cameras. Available at: http://www.regionofwaterloo.ca/en/safehealthycommunity/red_light_cameras.asp Accessed on: Jul. 10, 2017.

Retting, R. and Kyrychenko, S. (2002). Collision Reductions Associated with Red Light Camera Enforcement in Oxnard, California. Am. J. Public Health, Vol. 92, pp. 18221825.

Retting, R., Ferguson, S., and Farmer, C. (2007). Reducing Red Light Running Through Longer Yellow Signal Timing and Red Light Camera Enforcement: Results of a Field Investigation. Insurance Institute for Highway Safety, Arlington Virginia. 
Rosenbaum, P.R. and Rubin, D.B. (1983). The central role of the propensity score in observational studies for causal effects. Biometrika, 70(1): pp. 41-55.

Rosenbaum, P.R. and Rubin, D.B. (1985). Constructing a Control Group Using Multivariate Matched Sampling Methods that Incorporate the Propensity Score. The American Statistician, 39(1): pp. 33-38.

Rosenbaum, P.R. (2002).Observational Studies. 2nd ed. New York, NY: Springer.

Ruby, D. (2001). Assessment of Red Light Running Cameras in Fairfax County, Virginia, Project and Report submitted to the faculty of Virginia Polytechnic Institute and State University.

Sauerbrei, W., Royston, P. and Binder, H. (2007). Selection of important variables and determination of functional form for continuous predictors in multivariable model building. Statistics in Medicine, 26: 5512-5528. https://www.umassmed.edu/contentassets/a 7bd41506c5a4308b401e312a $7 \mathrm{ff59fc/sauerbri}$ e-royston-2007.pdf Accessed on: Jul. 10, 2017.

Sasidharan, L. and Donnell, E. (2013). Application of propensity scores and potential outcomes to estimate effectiveness of traffic safety countermeasures: Exploratory analysis using intersection lighting data, Accident Analysis \& Prevention, 50, 539-553.

Sayed, T., and de Leur, P. (2007). Evaluation of intersection safety camera program in Edmonton, Canada. Transportation Research Record: Journal of the Transportation Research Board No. 2009, Transportation Research Board of the National Academics, Washington, D.C., pp. 37-45.

Sayed, T. and de Leur, P. (2008). Collision Prediction Models for British Columbia. Prepared For: Engineering Branch - BC Ministry of Transportation \& Infrastructure.

Shankar, V., Milton, J. and Mannering, F. (1998). Modeling accident frequencies as zeroaltered probability processes: an empirical inquiry. Accident Analysis and Prevention, 29(6): pp. 829-837.

Shen, J., and Gan, A. (2003). Development of Crash Reduction Factors: Methods, Problems and Research Needs. Transportation Research Record 1840. pp. 50-56.

Shimizu, H. Desroches, P. (2015). Speed or Greed: Does Automated Traffic Enforcement Improve Safety or Generate Revenue? The Frontier Centre for Public Policy. Available at: https://fcpp.org/wp-content/uploads/2015/12/Shimizu-Desrochers-Speed-or-Greed.pdf Accessed on: Jul. 15, 2017.

Shin, K., and Washington, S. (2007). The impact of red light cameras on safety in Arizona. Accident Analysis and Prevention, 39: pp. 1212-1221.

Simpson, A. Kazakov, and L. Tasca. (1994). The Social Cost of Motor Vehicle Collisions in Ontario. SRO-94-101. Safety Research Office, Safety Policy Branch, Research and Development Branch, Ontario Ministry of Transportation.

Solomon, H. (2011). Red Light Camera Program and Other Traffic Safety Initiatives (PW11048) (City Wide). City of Hamilton - Public Works Department. Available at: http://www2.hamilton.ca/NR/rdonlyres/68C78372-9FB7-4472-9BF4-

DA86F5C33009/0/Jun20EDRMS_n180036_v1_8_3_PW11048.pdf Accessed on: Jul. 10, 2017.

Stata Manual. (2016). Tebalance overid. Available at: http://www.stata.com/manuals/tetebalanceoverid.pdf Accessed on: Jul. 10, 2017.

StataCorp. (2013). Stata Statistical Software: Release 13. College Station, TX: StataCorp LP. 
Statistical Consulting Group. (2007). Regression Models with Count Data. UCLA Academic Technology Services. Available at: http://www.ats.ucla.edu/stat/StataSE /seminars/count_presentation/count.htm Accessed on: Jul. 1, 2017.

Statistics Canada. (2017). Consumer Price Index, by province (Ontario). Available at: http://www.statcan.gc.ca/tables-tableaux/sumsom/101/cst01/econ09g-eng.htm Accessed on: Jul. 1, 2017.

Stuart, A., Lee, B. and Leacy, F. (2013). Prognostic Score-Based Balance Measures Can Be a Useful Diagnostic for Propensity Scores in Comparative Effectiveness Research. Journal of Clinical Epidemiology, 66:84-90.

Synectics Transportation Consultants. (2003). Evaluation of the Red-light Camera Enforcement Pilot Project: Final Technical Report. Ontario Ministry of Transportation, Toronto. Available at: http://www.thenewspaper.com/rlc/docs/2003-ontario.pdf Accessed Jul. 13, 2017.

Transport Canada. (2011). Road Safety in Canada. Motor Vehicle Safety with support from the Public Health Agency of Canada. TP 15145 E. Cat. T46-54/1-2011E. ISBN 978-1-10018621-4

Torres-Reyna, O. (2007). Panel Data Analysis Fixed and Random Effects using Stata (v. 4.2). Princeton University. Available at: https://www.princeton.edu/ otorres/Panel101.pdf Accessed on: Jul. 9, 2017.

Tumilty, R. (2017). "Ottawa lacks recent data on red-light cameras." Metro News. Metro Published on Mon Mar 27 2017. Available at: http://www.metronews.ca/news/ottawa/2017/03/26/ottawa-lacks-recent-data-on-red-lightcameras-.html Accessed on: Jul. 10, 2017.

UCLA. (2011) StataSE Data Analysis Examples Zero-inflated Negative Binomial Regression. UCLA: Statistical Consulting Group. Available at: http://www.ats.ucla.edu/stat/sas/notes2/ Accessed on: Jul. 10, 2017.

Vanlaar, W., Robertson, R., and Marcoux, K. (2011). Evaluation of the photo enforcement safety program of the city of Winnipeg: Final report. Traffic Injury Research Foundation. ISBN 978-926857-16-9.

Vodden, K., Smith, D., Eaton, F. and Mayhew, D. (2007). Analysis and Estimation of the Social Cost of Motor Vehicle Collisions in Ontario. Transport Canada. N0779. TP 14800F, 204 p. N.B. http://blog.caasco.com/wp-content/uploads/2013/08/tp14800e.pdf Accessed on: Jul. 12, 2017.

Vuong, Q. H. (1989). Likelihood ratio tests for model selection and non-nested hypotheses. Econometrica, 57: pp.307-333.

Washington, S. and Shin K. (2005). The Impact of Red Light Cameras (Automated Enforcement) on Safety in Arizona. Phoenix, AZ: Arizona Department of Transportation. https://ntl.bts.gov/lib/25000/25000/25016/AZ550.pdf Accessed on: Jul. 10, 2017.

Weber, P. (2015). Traffic Signals or roundabout at this unusual intersection? Paper prepared for presentation at the Geometric Design - Emerging Issues Session of the 2015 Annual Conference of the Transportation Association of Canada (TAC). Charlottetown, PEI. Available at: http://conf.tac-atc.ca/english/annualconference/tac2015/s7/weber.pdf Accessed on: Jul. 15, 2017.

Williams, R. (2012). Using the margins command to estimate and interpret adjusted predictions and marginal effects The StataSE Journal. 12, Number 2, pp. 308-331. Available at: 
http://www.econ.uzh.ch/dam/jcr:00000000-5766-84b0-ffff-ffffa80635e1/sj12-

2.pdf\#page $=148$ Accessed on: Jul. 13, 2017.

Williams, R. (2016). Panel Data 4: Fixed Effects vs Random Effects Models. University of Notre Dame. Available at: https://www3.nd.edu/ rwilliam/stats3/Panel04FixedVsRandom.pdf Accessed on: Jul. 13, 2017.

Willing, J. (2016). " 4,900 blew camera-guarded red lights in 2015 without getting tickets". Ottawa Citizen. Available at: http://ottawacitizen.com/storyline/4900-blew-cameraguarded-red-lights-in-2015-without-getting-tickets Accessed on: Jul. 10, 2017.

Wilson, C., Willis, C., Hendrikz, J. and Bellamy, N. (2006). Speed enforcement detection devices for preventing road traffic injuries. Cochrane Database of Systematic Reviews, Issue 2. Art. No.: CD004607. DOI: 10.1002/14651858.CD004607.pub2 Accessed on: Jul. $10,2017$.

Wong, T. (2014). Lights, camera, legal action! The effectiveness of red light cameras on collisions in Los Angeles. Transportation Research Part A: Policy and Practice, 2014; 69: pp. 165-182.

Wooldridge, J.M. (2007). Inverse probability weighted estimation for general missing data problems. Journal of Econometrics, 141: pp. 1281-1301. Available at: https://doi.org/10.1016/j.jeconom.2007.02.002 Accessed on: Jul. 10, 2017.

Wooldridge, J.M. (2010). Econometric Analysis of Cross Section and Panel Data, 2nd edn. MIT Press, Cambridge, MA.

Yaacob, W., Lazim, M. and Wah, Y. (2011). Applying Fixed Effects Panel Count Model to Examine Road Accident Occurrence. Journal of Applied Sciences, 11: 1185-1191. Available at: http://scialert.net/fulltext/?doi=jas.2011.1185.1191\&org=11 Accessed on: Jul. 15, 2017. 


\section{Appendix A - Descriptive Data}

Table A - Non-RLC intersections

\begin{tabular}{|l|l|}
\hline Number & \multicolumn{1}{|c|}{ Non-RLC Intersection } \\
\hline 1 & ALBION RD \& HUNT CLUB RD \\
\hline 2 & BANK ST \& CATHERINE ST \\
\hline 3 & BANK ST \& RIVERSIDE DR S \\
\hline 4 & BASELINE RD \& HERON RD \\
\hline 5 & BLAIR RD \& OGILVIE RD \\
\hline 6 & BRIDLE PATH DR \& HUNT CLUB RD \\
\hline 7 & CARLING AVE \& MERIVALE RD \\
\hline 8 & COLONEL BY DR \& HAWTHORNE AVE \\
\hline 9 & CONROY RD \& HUNT CLUB RD \\
\hline 10 & GREENBANK RD \& STRANDHERD DR \\
\hline 11 & INDUSTRIAL AVE \& ST. LAURENT BLVD \\
\hline 12 & KING EDWARD AVE \& MURRAY ST \\
\hline 13 & LEMIEUX ST \& ST. LAURENT BLVD \\
\hline 14 & PINECREST RD \& RICHMOND RD \\
\hline
\end{tabular}

Table B - Demographics of Ottawa, Ontario, Canada

\begin{tabular}{|c|c|c|c|}
\hline Year & Population & $\begin{array}{c}\text { Registered } \\
\text { Vehicles }\end{array}$ & $\begin{array}{c}\text { Licensed } \\
\text { Drivers }\end{array}$ \\
\hline \hline 1999 & 769,511 & 413,892 & 560,852 \\
\hline 2000 & 786,975 & 430,547 & 566,517 \\
\hline 2001 & 806,560 & 441,625 & 572,240 \\
\hline 2002 & 817,585 & 441,169 & 578,020 \\
\hline 2003 & 825,783 & 445,120 & 588,694 \\
\hline 2004 & 830,910 & 446,250 & 589,816 \\
\hline 2005 & 836,505 & 453,168 & 594,640 \\
\hline 2006 & 845,917 & 462,406 & 613,700 \\
\hline 2007 & 855,257 & 473,384 & 620,654 \\
\hline 2008 & 868,095 & 485,973 & 626,000 \\
\hline 2009 & 883,741 & 498,120 & 635,000 \\
\hline 2010 & 899,016 & 508,389 & 635,112 \\
\hline 2011 & 912,248 & 515,784 & 653,324 \\
\hline 2012 & 924,404 & 520,942 & 659,857 \\
\hline 2013 & 934,300 & 526,151 & 666,456 \\
\hline
\end{tabular}


Table C - Summary of collision database by impact type per intersection per year

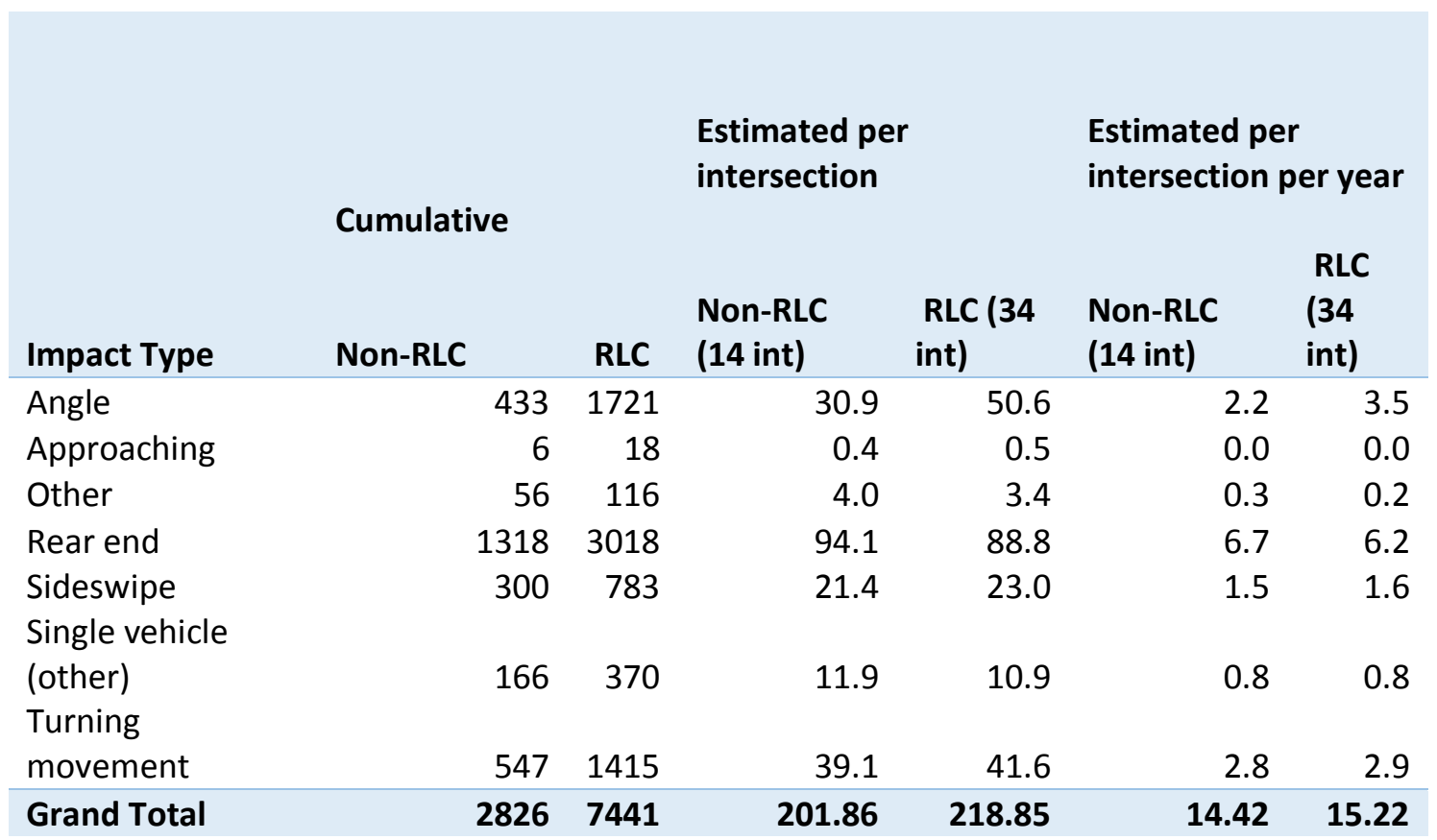

\section{T-TESTS}

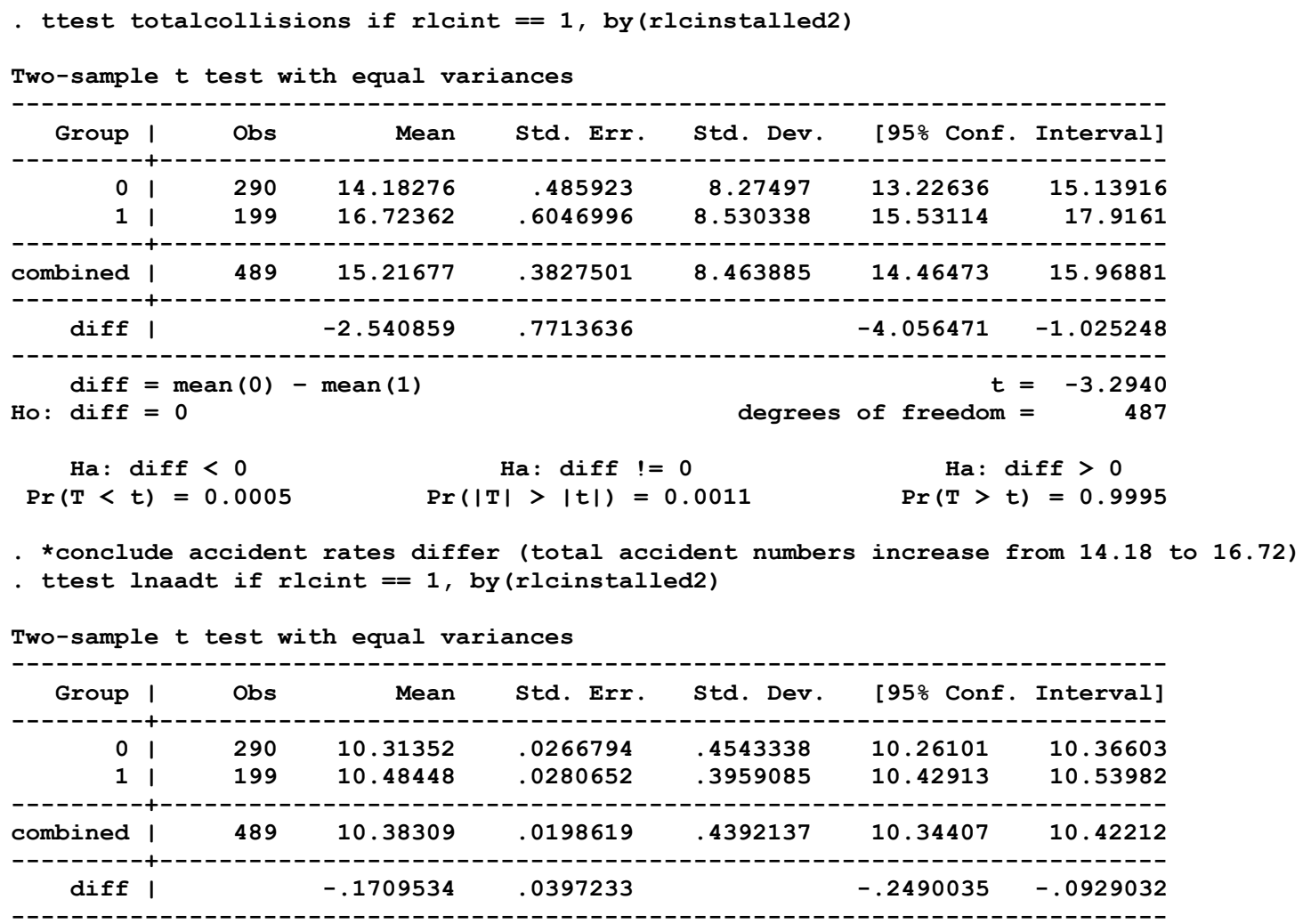




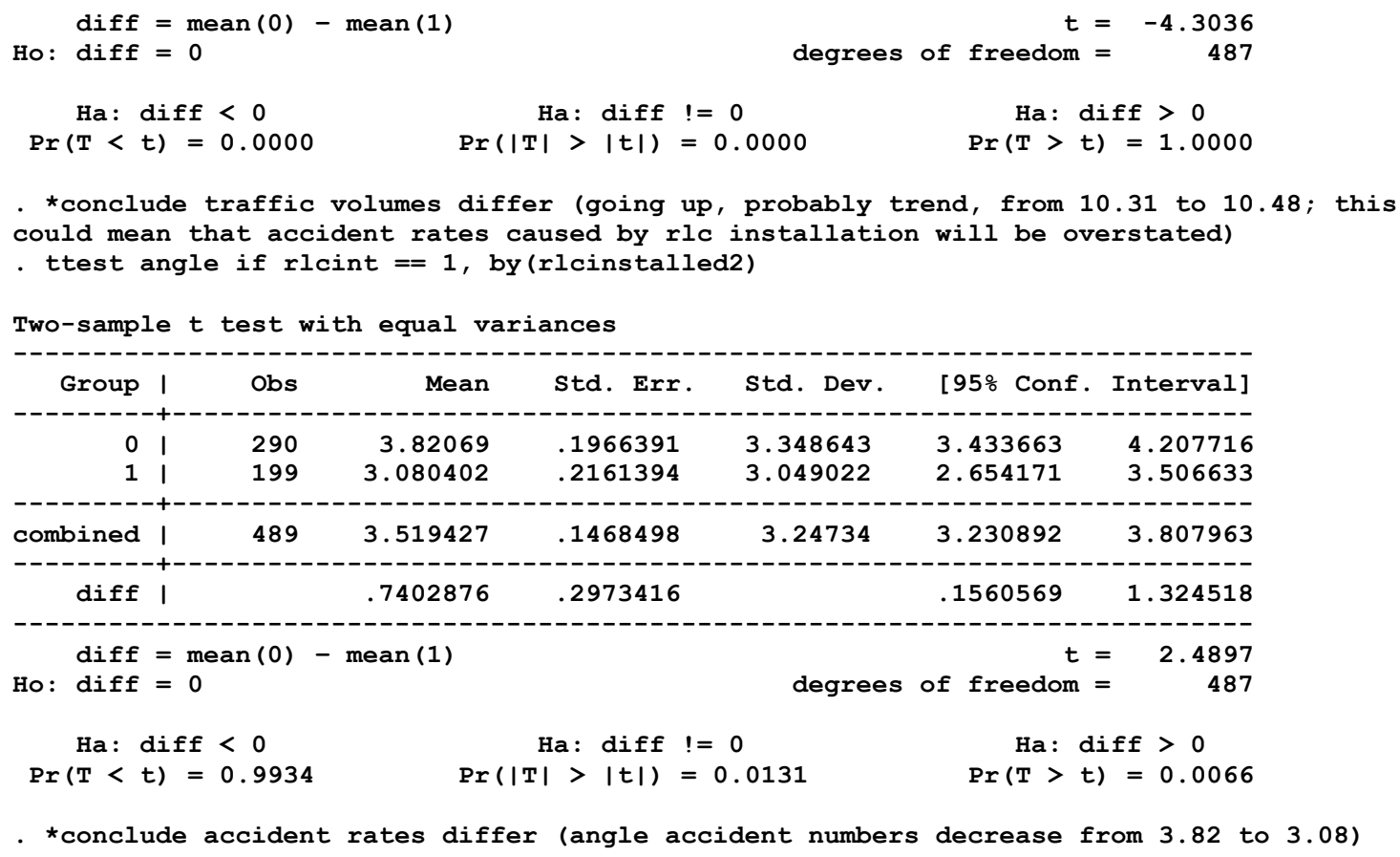

- ttest turningmovement if rlcint $==1$, by (rlcinstalled2)

Two-sample $t$ test with equal variances

\begin{tabular}{|c|c|c|c|c|c|c|}
\hline Group I & Obs & Mean & Std. Err. & Std. Dev. & [95\% Conf. & Interval] \\
\hline 01 & 290 & 3.034483 & .1889962 & 3.218489 & 2.662499 & 3.406466 \\
\hline $1 i$ & 199 & 2.688442 & .2333041 & 3.29116 & 2.228362 & 3.148522 \\
\hline ombined I & 489 & 2.893661 & .1469411 & 3.24936 & 2.604945 & 3.182376 \\
\hline diff | & & .3460405 & .2990032 & & -.2414549 & .933536 \\
\hline o: $\begin{array}{l}\text { diff }= \\
\operatorname{diff}=\end{array}$ & (0) & $\operatorname{lean}(1)$ & & degree & of freedom & $\begin{array}{r}1.1573 \\
487\end{array}$ \\
\hline
\end{tabular}
Ha: diff $<0$ Ha: diff $!=0 \quad$ Ha: diff $>0$
$\operatorname{Pr}(T<t)=0.8761 \quad \operatorname{Pr}(|T|>|t|)=0.2477 \quad \operatorname{Pr}(T>t)=0.1239$

. *conclude accident rates DO NOT differ (TM accident numbers decrease marginally from 3.03 ro 2.69 but not significant)

- ttest rearend if rlcint $==1$, by(rlcinstalled2)

Two-sample $t$ test with equal variances

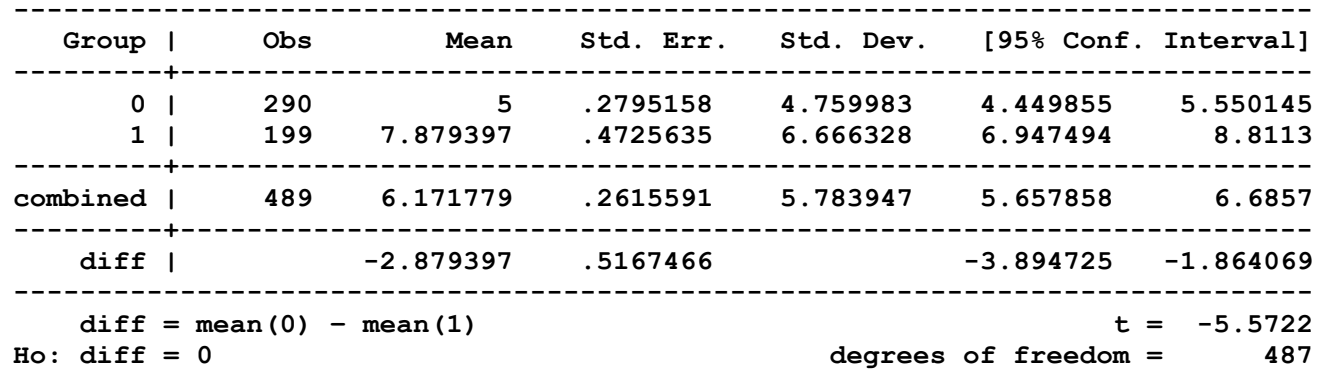

Ha: diff $<0$

$\operatorname{Pr}(T<t)=0.0000$
Ha: diff $!=0$

$\operatorname{Pr}(|\mathrm{T}|>|\mathrm{t}|)=0.0000$
Ha: diff $>0$

$\operatorname{Pr}(T>t)=1.0000$ 


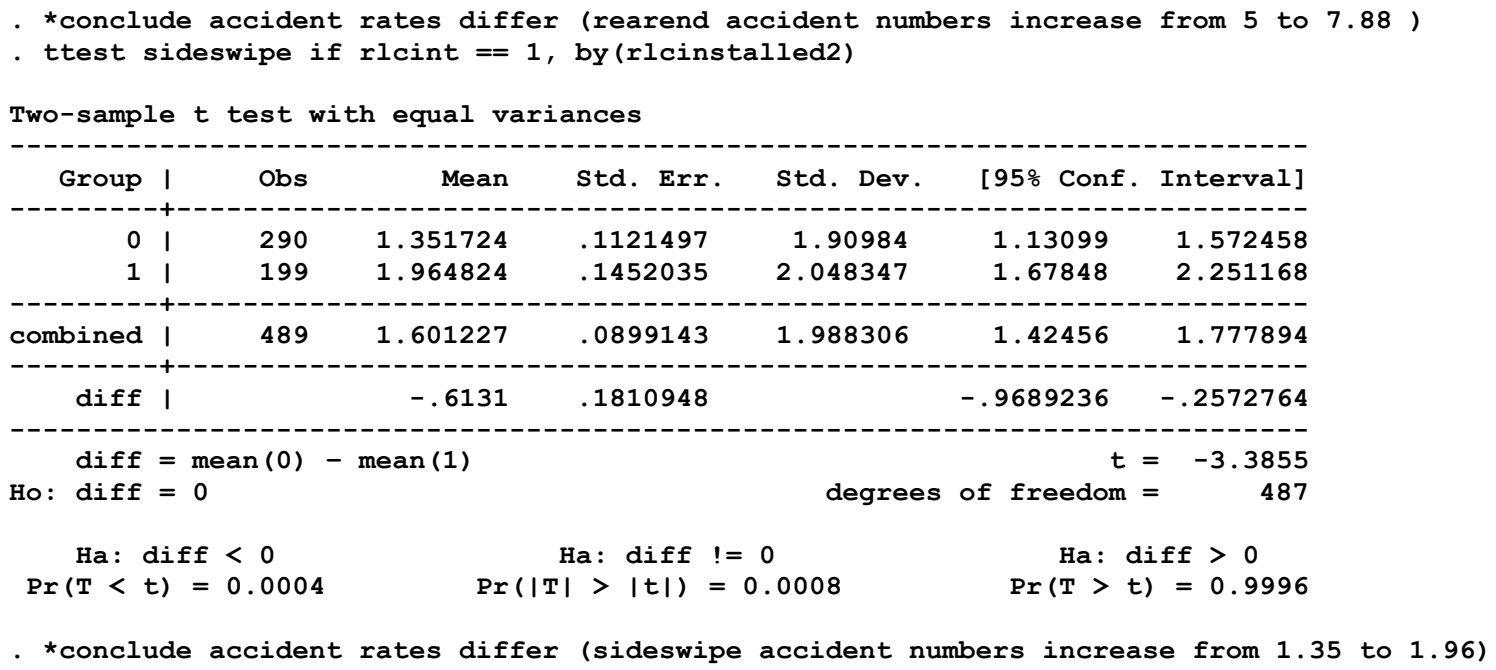

\section{Summary of observed collisions - BEFORE and AFTER}

\begin{tabular}{lrrr} 
Average of Totalcollisions & & \multicolumn{2}{c}{$\begin{array}{l}\text { Grand } \\
\text { Tefor }\end{array}$} \\
\hline NON-RLC & $\mathbf{1 4 . 4 2}$ & $\mathbf{1 4 . 4 2}$ \\
\hline ALBION RD \& HUNT CLUB RD & 11.79 & 11.79 \\
BANK ST \& CATHERINE ST & 12.50 & 12.50 \\
BANK ST \& RIVERSIDE DR S & 12.50 & 12.50 \\
BASELINE RD \& HERON RD & 13.07 & & 13.07 \\
BLAIR RD \& OGILVIE RD & 18.07 & & 18.07 \\
BRIDLE PATH DR \& HUNT CLUB RD & 16.29 & & 16.29 \\
CARLING AVE \& MERIVALE RD & 12.07 & & 12.07 \\
COLONEL BY DR \& HAWTHORNE AVE & 10.14 & & 10.14 \\
CONROY RD \& HUNT CLUB RD & 14.29 & & 14.29 \\
GREENBANK RD \& STRANDHERD DR & 16.93 & & 16.93 \\
INDUSTRIAL AVE \& ST. LAURENT BLVD & 24.29 & & 24.29 \\
KING EDWARD AVE \& MURRAY ST & 11.21 & & 11.21 \\
LEMIEUX ST \& ST. LAURENT BLVD & 13.57 & & 13.57 \\
PINECREST RD \& RICHMOND RD & 15.14 & & 15.14 \\
RLC & 14.18 & 16.72 & 15.22 \\
\hline ALBERT ST \& BOOTH ST & 12.91 & 12.75 & 12.87 \\
ALBERT ST \& KENT ST (OTTAWA) & 11.50 & 12.00 & 11.93 \\
AVIATION PKWY \& OGILVIE RD & 16.64 & 13.75 & 15.87 \\
BANK ST \& HERON RD & 22.45 & 22.50 & 22.47 \\
BANK ST \& HUNT CLUB RD & 19.67 & 25.60 & 21.79 \\
BANK ST \& RIVERSIDE DR N & 16.22 & 15.80 & 16.07 \\
BASELINE RD \& MERIVALE RD & 26.22 & 16.20 & 22.64
\end{tabular}


BELFAST RD \& ST. LAURENT BLVD BERRIGAN DR \& GREENBANK RD

BLAIR RD \& BLAIR RD RAMP 36 BOOTH ST \& OTTAWA RIVER PKWY BRONSON AVE \& CARLING AVE BRONSON AVE \& COMMISSIONER ST CAMPEAU DR \& MARCH RD CARLING AVE \& ISLAND PARK DR CARLING AVE \& RICHMOND RD CATHERINE ST \& HWY 417 O'CONN CATHERINE ST \& KENT ST CHARLEMAGNE BLVD N \& TENTH LINE RD CONROY RD \& LORRY GREENBERG DR COVENTRY RD \& ST. LAURENT BLVD CYRVILLE RD \& INNES RD DRIVEWAY (THE) \& ISLAND PARK DR DUFORD DR \& ST. JOSEPH BLVD EAGLESON RD \& HAZELDEAN RD FISHER AVE \& MEADOWLANDS DR HAWTHORNE AVE \& QUEEN ELIZABETH DRWY HERON RD \& RIVERSIDE DR JOCKVALE RD \& STRANDHERD DR KING EDWARD AVE \& ST. ANDREW ST KING EDWARD AVE \& ST. PATRICK ST MEADOWLANDS DR \& MERIVALE RD RUSSELL RD \& WALKLEY RD SAUNDERSON DR \& SMYTH RD

$\begin{array}{rrr}21.89 & 28.00 & 24.07 \\ 9.73 & 11.25 & 10.13 \\ 16.91 & 25.25 & 19.13 \\ 9.78 & 10.40 & 10.00 \\ 18.00 & 15.15 & 15.36 \\ 14.00 & 8.75 & 12.60 \\ 20.44 & 18.60 & 19.79 \\ 10.22 & 8.80 & 9.71 \\ 11.00 & 14.08 & 13.86 \\ 17.78 & 12.00 & 15.71 \\ 20.89 & 23.00 & 21.64 \\ 14.56 & 20.00 & 16.50 \\ 3.00 & 2.50 & 2.87 \\ 21.00 & 24.25 & 23.79 \\ 15.18 & 14.50 & 15.00 \\ 8.55 & 8.50 & 8.53 \\ 8.45 & 7.00 & 8.07 \\ 18.50 & 16.17 & 16.50 \\ 13.27 & 12.50 & 13.07 \\ 8.45 & 9.00 & 8.60 \\ 27.50 & 29.33 & 29.07 \\ 3.82 & 5.50 & 4.27 \\ 4.00 & 1.50 & 3.64 \\ 25.50 & 9.00 & 23.14 \\ 20.00 & 23.00 & 22.57 \\ 21.89 & 30.40 & 24.93 \\ 4.17 & 5.50 & 4.36 \\ 14.28 & 16.72 & 14.99\end{array}$

\begin{tabular}{|c|c|c|c|}
\hline Average of Rearend & BEFORE & AFTER & $\begin{array}{l}\text { Grand } \\
\text { Total }\end{array}$ \\
\hline NON-RLC & 6.72 & & 6.72 \\
\hline ALBION RD \& HUNT CLUB RD & 5.86 & & 5.86 \\
\hline BANK ST \& CATHERINE ST & 3.07 & & 3.07 \\
\hline BANK ST \& RIVERSIDE DR S & 3.86 & & 3.86 \\
\hline BASELINE RD \& HERON RD & 8.50 & & 8.50 \\
\hline BLAIR RD \& OGILVIE RD & 11.00 & & 11.00 \\
\hline BRIDLE PATH DR \& HUNT CLUB RD & 8.64 & & 8.64 \\
\hline CARLING AVE \& MERIVALE RD & 3.21 & & 3.21 \\
\hline COLONEL BY DR \& HAWTHORNE AVE & 2.43 & & 2.43 \\
\hline CONROY RD \& HUNT CLUB RD & 9.21 & & 9.21 \\
\hline
\end{tabular}




\begin{tabular}{|c|c|c|c|}
\hline GREENBANK RD \& STRANDHERD DR & 6.57 & & 6.57 \\
\hline INDUSTRIAL AVE \& ST. LAURENT BLVD & 14.64 & & 14.64 \\
\hline KING EDWARD AVE \& MURRAY ST & 4.50 & & 4.50 \\
\hline LEMIEUX ST \& ST. LAURENT BLVD & 5.93 & & 5.93 \\
\hline PINECREST RD \& RICHMOND RD & 6.71 & & 6.71 \\
\hline RLC & 5.00 & 7.88 & 6.17 \\
\hline ALBERT ST \& BOOTH ST & 3.55 & 3.25 & 3.47 \\
\hline ALBERT ST \& KENT ST (OTTAWA) & 1.00 & 0.33 & 0.43 \\
\hline AVIATION PKWY \& OGILVIE RD & 4.73 & 6.75 & 5.27 \\
\hline BANK ST \& HERON RD & 8.55 & 11.25 & 9.27 \\
\hline BANK ST \& HUNT CLUB RD & 10.33 & 15.00 & 12.00 \\
\hline BANK ST \& RIVERSIDE DR N & 4.11 & 5.20 & 4.50 \\
\hline BASELINE RD \& MERIVALE RD & 12.89 & 11.80 & 12.50 \\
\hline BELFAST RD \& ST. LAURENT BLVD & 5.56 & 9.40 & 6.93 \\
\hline BERRIGAN DR \& GREENBANK RD & 6.09 & 7.00 & 6.33 \\
\hline BLAIR RD \& BLAIR RD RAMP 36 & 10.36 & 14.00 & 11.33 \\
\hline BOOTH ST \& OTTAWA RIVER PKWY & 2.78 & 3.40 & 3.00 \\
\hline BRONSON AVE \& CARLING AVE & 5.00 & 4.69 & 4.71 \\
\hline BRONSON AVE \& COMMISSIONER ST & 1.55 & 1.00 & 1.40 \\
\hline CAMPEAU DR \& MARCH RD & 12.89 & 11.00 & 12.21 \\
\hline CARLING AVE \& ISLAND PARK DR & 1.11 & 1.60 & 1.29 \\
\hline CARLING AVE \& RICHMOND RD & 6.00 & 7.46 & 7.36 \\
\hline CATHERINE ST \& HWY 417 O'CONN & 2.67 & 1.60 & 2.29 \\
\hline CATHERINE ST \& KENT ST & 0.78 & 1.60 & 1.07 \\
\hline CHARLEMAGNE BLVD N \& TENTH LINE RD & 6.22 & 12.80 & 8.57 \\
\hline CONROY RD \& LORRY GREENBERG DR & 0.55 & 1.25 & 0.73 \\
\hline COVENTRY RD \& ST. LAURENT BLVD & 7.50 & 12.67 & 11.93 \\
\hline CYRVILLE RD \& INNES RD & 8.00 & 9.25 & 8.33 \\
\hline DRIVEWAY (THE) \& ISLAND PARK DR & 0.73 & 1.50 & 0.93 \\
\hline DUFORD DR \& ST. JOSEPH BLVD & 2.91 & 2.25 & 2.73 \\
\hline EAGLESON RD \& HAZELDEAN RD & 9.50 & 10.92 & 10.71 \\
\hline FISHER AVE \& MEADOWLANDS DR & 2.27 & 1.75 & 2.13 \\
\hline HAWTHORNE AVE \& QUEEN ELIZABETH DRWY & 2.18 & 3.25 & 2.47 \\
\hline HERON RD \& RIVERSIDE DR & 17.50 & 22.08 & 21.43 \\
\hline JOCKVALE RD \& STRANDHERD DR & 0.64 & 2.25 & 1.07 \\
\hline KING EDWARD AVE \& ST. ANDREW ST & 2.92 & 1.00 & 2.64 \\
\hline KING EDWARD AVE \& ST. PATRICK ST & 6.92 & 3.00 & 6.36 \\
\hline MEADOWLANDS DR \& MERIVALE RD & 10.50 & 9.83 & 9.93 \\
\hline RUSSELL RD \& WALKLEY RD & 12.56 & 20.00 & 15.21 \\
\hline SAUNDERSON DR \& SMYTH RD & 0.75 & 3.00 & 1.07 \\
\hline Grand Total & 5.70 & 7.88 & 6.33 \\
\hline
\end{tabular}




\begin{tabular}{|c|c|c|c|}
\hline Average of Angle & BEFORE & AFTER & $\begin{array}{l}\text { Grand } \\
\text { Total }\end{array}$ \\
\hline NON-RLC & 2.21 & & 2.21 \\
\hline ALBION RD \& HUNT CLUB RD & 1.29 & & 1.29 \\
\hline BANK ST \& CATHERINE ST & 3.50 & & 3.50 \\
\hline BANK ST \& RIVERSIDE DR S & 4.57 & & 4.57 \\
\hline BASELINE RD \& HERON RD & 1.43 & & 1.43 \\
\hline BLAIR RD \& OGILVIE RD & 1.00 & & 1.00 \\
\hline BRIDLE PATH DR \& HUNT CLUB RD & 1.07 & & 1.07 \\
\hline CARLING AVE \& MERIVALE RD & 2.57 & & 2.57 \\
\hline COLONEL BY DR \& HAWTHORNE AVE & 4.71 & & 4.71 \\
\hline CONROY RD \& HUNT CLUB RD & 1.50 & & 1.50 \\
\hline GREENBANK RD \& STRANDHERD DR & 1.43 & & 1.43 \\
\hline INDUSTRIAL AVE \& ST. LAURENT BLVD & 1.57 & & 1.57 \\
\hline KING EDWARD AVE \& MURRAY ST & 2.36 & & 2.36 \\
\hline LEMIEUX ST \& ST. LAURENT BLVD & 1.79 & & 1.79 \\
\hline PINECREST RD \& RICHMOND RD & 2.14 & & 2.14 \\
\hline RLC & 3.82 & 3.08 & 3.52 \\
\hline ALBERT ST \& BOOTH ST & 4.36 & 2.75 & 3.93 \\
\hline ALBERT ST \& KENT ST (OTTAWA) & 10.50 & 9.58 & 9.71 \\
\hline AVIATION PKWY \& OGILVIE RD & 2.36 & 1.50 & 2.13 \\
\hline BANK ST \& HERON RD & 3.00 & 1.25 & 2.53 \\
\hline BANK ST \& HUNT CLUB RD & 3.78 & 3.40 & 3.64 \\
\hline BANK ST \& RIVERSIDE DR N & 7.78 & 6.40 & 7.29 \\
\hline BASELINE RD \& MERIVALE RD & 3.33 & 1.40 & 2.64 \\
\hline BELFAST RD \& ST. LAURENT BLVD & 3.44 & 2.20 & 3.00 \\
\hline BERRIGAN DR \& GREENBANK RD & 1.73 & 1.50 & 1.67 \\
\hline BLAIR RD \& BLAIR RD RAMP 36 & 3.73 & 3.75 & 3.73 \\
\hline BOOTH ST \& OTTAWA RIVER PKWY & 5.00 & 3.20 & 4.36 \\
\hline BRONSON AVE \& CARLING AVE & 4.00 & 0.62 & 0.86 \\
\hline BRONSON AVE \& COMMISSIONER ST & 5.36 & 4.50 & 5.13 \\
\hline CAMPEAU DR \& MARCH RD & 3.33 & 3.20 & 3.29 \\
\hline CARLING AVE \& ISLAND PARK DR & 5.11 & 3.00 & 4.36 \\
\hline CARLING AVE \& RICHMOND RD & 3.00 & 3.54 & 3.50 \\
\hline CATHERINE ST \& HWY 417 O'CONN & 11.78 & 7.20 & 10.14 \\
\hline CATHERINE ST \& KENT ST & 5.78 & 7.40 & 6.36 \\
\hline CHARLEMAGNE BLVD N \& TENTH LINE RD & 3.00 & 2.40 & 2.79 \\
\hline CONROY RD \& LORRY GREENBERG DR & 1.18 & 0.25 & 0.93 \\
\hline COVENTRY RD \& ST. LAURENT BLVD & 7.50 & 4.25 & 4.71 \\
\hline CYRVILLE RD \& INNES RD & 2.91 & 2.00 & 2.67 \\
\hline DRIVEWAY (THE) \& ISLAND PARK DR & 2.82 & 2.75 & 2.80 \\
\hline DUFORD DR \& ST. JOSEPH BLVD & 2.27 & 1.25 & 2.00 \\
\hline
\end{tabular}




\begin{tabular}{llll} 
EAGLESON RD \& HAZELDEAN RD & 2.50 & 1.58 & 1.71 \\
FISHER AVE \& MEADOWLANDS DR & 3.73 & 1.75 & 3.20 \\
HAWTHORNE AVE \& QUEEN ELIZABETH DRWY & 3.64 & 4.25 & 3.80 \\
HERON RD \& RIVERSIDE DR & 7.00 & 2.83 & 3.43 \\
JOCKVALE RD \& STRANDHERD DR & 1.55 & 1.00 & 1.40 \\
KING EDWARD AVE \& ST. ANDREW ST & 0.33 & 0.00 & 0.29 \\
KING EDWARD AVE \& ST. PATRICK ST & 8.00 & 1.00 & 7.00 \\
MEADOWLANDS DR \& MERIVALE RD & 3.50 & 1.33 & 1.64 \\
RUSSELL RD \& WALKLEY RD & 2.56 & 1.60 & 2.21 \\
SAUNDERSON DR \& SMYTH RD & 1.67 & 0.50 & 1.50 \\
\hline Grand Total & $\mathbf{3 . 1 7}$ & $\mathbf{3 . 0 8}$ & $\mathbf{3 . 1 4}$
\end{tabular}

\begin{tabular}{|c|c|c|c|}
\hline Average of TurningMovement & BEFORE & AFTER & $\begin{array}{l}\text { Grand } \\
\text { Total }\end{array}$ \\
\hline NON-RLC & 2.79 & & 2.79 \\
\hline ALBION RD \& HUNT CLUB RD & 3.50 & & 3.50 \\
\hline BANK ST \& CATHERINE ST & 2.64 & & 2.64 \\
\hline BANK ST \& RIVERSIDE DR S & 0.64 & & 0.64 \\
\hline BASELINE RD \& HERON RD & 0.36 & & 0.36 \\
\hline BLAIR RD \& OGILVIE RD & 2.00 & & 2.00 \\
\hline BRIDLE PATH DR \& HUNT CLUB RD & 3.93 & & 3.93 \\
\hline CARLING AVE \& MERIVALE RD & 3.21 & & 3.21 \\
\hline COLONEL BY DR \& HAWTHORNE AVE & 1.93 & & 1.93 \\
\hline CONROY RD \& HUNT CLUB RD & 1.93 & & 1.93 \\
\hline GREENBANK RD \& STRANDHERD DR & 6.36 & & 6.36 \\
\hline INDUSTRIAL AVE \& ST. LAURENT BLVD & 3.36 & & 3.36 \\
\hline KING EDWARD AVE \& MURRAY ST & 0.93 & & 0.93 \\
\hline LEMIEUX ST \& ST. LAURENT BLVD & 3.57 & & 3.57 \\
\hline PINECREST RD \& RICHMOND RD & 4.71 & & 4.71 \\
\hline RLC & 3.03 & 2.69 & 2.89 \\
\hline ALBERT ST \& BOOTH ST & 2.91 & 4.50 & 3.33 \\
\hline ALBERT ST \& KENT ST (OTTAWA) & 0.00 & 0.58 & 0.50 \\
\hline AVIATION PKWY \& OGILVIE RD & 7.55 & 3.50 & 6.47 \\
\hline BANK ST \& HERON RD & 4.45 & 2.00 & 3.80 \\
\hline BANK ST \& HUNT CLUB RD & 1.78 & 1.40 & 1.64 \\
\hline BANK ST \& RIVERSIDE DR N & 1.33 & 1.00 & 1.21 \\
\hline BASELINE RD \& MERIVALE RD & 5.33 & 0.00 & 3.43 \\
\hline BELFAST RD \& ST. LAURENT BLVD & 8.89 & 11.20 & 9.71 \\
\hline BERRIGAN DR \& GREENBANK RD & 0.91 & 2.25 & 1.27 \\
\hline BLAIR RD \& BLAIR RD RAMP 36 & 2.00 & 5.25 & 2.87 \\
\hline BOOTH ST \& OTTAWA RIVER PKWY & 1.33 & 1.80 & 1.50 \\
\hline
\end{tabular}




$\begin{array}{llll}\text { BRONSON AVE \& CARLING AVE } & 6.00 & 5.38 & 5.43 \\ \text { BRONSON AVE \& COMMISSIONER ST } & 4.91 & 2.00 & 4.13 \\ \text { CAMPEAU DR \& MARCH RD } & 1.67 & 2.60 & 2.00 \\ \text { CARLING AVE \& ISLAND PARK DR } & 3.11 & 2.20 & 2.79 \\ \text { CARLING AVE \& RICHMOND RD } & 1.00 & 0.62 & 0.64 \\ \text { CATHERINE ST \& HWY 417 O'CONN } & 0.11 & 0.60 & 0.29 \\ \text { CATHERINE ST \& KENT ST } & 9.44 & 8.80 & 9.21 \\ \text { CHARLEMAGNE BLVD N \& TENTH LINE RD } & 4.00 & 3.20 & 3.71 \\ \text { CONROY RD \& LORRY GREENBERG DR } & 0.73 & 0.75 & 0.73 \\ \text { COVENTRY RD \& ST. LAURENT BLVD } & 2.00 & 1.08 & 1.21 \\ \text { CYRVILLE RD \& INNES RD } & 0.91 & 1.25 & 1.00 \\ \text { DRIVEWAY (THE) \& ISLAND PARK DR } & 4.45 & 3.75 & 4.27 \\ \text { DUFORD DR \& ST. JOSEPH BLVD } & 2.36 & 1.75 & 2.20 \\ \text { EAGLESON RD \& HAZELDEAN RD } & 2.00 & 0.83 & 1.00 \\ \text { FISHER AVE \& MEADOWLANDS DR } & 5.36 & 5.00 & 5.27 \\ \text { HAWTHORNE AVE \& QUEEN ELIZABETH DRWY } & 1.64 & 0.50 & 1.33 \\ \text { HERON RD \& RIVERSIDE DR } & 1.50 & 1.75 & 1.71 \\ \text { JOCKVALE RD \& STRANDHERD DR } & 1.09 & 1.50 & 1.20 \\ \text { KING EDWARD AVE \& ST. ANDREW ST } & 0.17 & 0.00 & 0.14 \\ \text { KING EDWARD AVE \& ST. PATRICK ST } & 4.42 & 1.00 & 3.93 \\ \text { MEADOWLANDS DR \& MERIVALE RD } & 5.00 & 8.00 & 7.57 \\ \text { RUSSELL RD \& WALKLEY RD } & 1.67 & 1.40 & 1.57 \\ \text { SAUNDERSON DR \& SMYTH RD } & 1.42 & 0.50 & 1.29 \\ \text { Grand TotaI } & \mathbf{2 . 9 4} & \mathbf{2 . 6 9} & \mathbf{2 . 8 6}\end{array}$

\begin{tabular}{|c|c|c|c|}
\hline Average of Sideswipe & BEFORE & AFTER & $\begin{array}{l}\text { Grand } \\
\text { Total }\end{array}$ \\
\hline NON-RLC & 1.53 & & 1.53 \\
\hline ALBION RD \& HUNT CLUB RD & 0.64 & & 0.64 \\
\hline BANK ST \& CATHERINE ST & 2.00 & & 2.00 \\
\hline BANK ST \& RIVERSIDE DR S & 2.57 & & 2.57 \\
\hline BASELINE RD \& HERON RD & 1.79 & & 1.79 \\
\hline BLAIR RD \& OGILVIE RD & 2.86 & & 2.86 \\
\hline BRIDLE PATH DR \& HUNT CLUB RD & 0.71 & & 0.71 \\
\hline CARLING AVE \& MERIVALE RD & 1.71 & & 1.71 \\
\hline COLONEL BY DR \& HAWTHORNE AVE & 0.29 & & 0.29 \\
\hline CONROY RD \& HUNT CLUB RD & 0.64 & & 0.64 \\
\hline GREENBANK RD \& STRANDHERD DR & 1.00 & & 1.00 \\
\hline INDUSTRIAL AVE \& ST. LAURENT BLVD & 3.07 & & 3.07 \\
\hline
\end{tabular}




\begin{tabular}{|c|c|c|c|}
\hline KING EDWARD AVE \& MURRAY ST & 2.14 & & 2.14 \\
\hline LEMIEUX ST \& ST. LAURENT BLVD & 1.36 & & 1.36 \\
\hline PINECREST RD \& RICHMOND RD & 0.64 & & 0.64 \\
\hline RLC & 1.35 & 1.96 & 1.60 \\
\hline ALBERT ST \& BOOTH ST & 0.73 & 2.00 & 1.07 \\
\hline ALBERT ST \& KENT ST (OTTAWA) & 0.00 & 0.75 & 0.64 \\
\hline AVIATION PKWY \& OGILVIE RD & 0.82 & 0.50 & 0.73 \\
\hline BANK ST \& HERON RD & 4.82 & 6.00 & 5.13 \\
\hline BANK ST \& HUNT CLUB RD & 3.00 & 5.00 & 3.71 \\
\hline BANK ST \& RIVERSIDE DR N & 2.00 & 2.60 & 2.21 \\
\hline BASELINE RD \& MERIVALE RD & 2.44 & 1.40 & 2.07 \\
\hline BELFAST RD \& ST. LAURENT BLVD & 2.00 & 3.80 & 2.64 \\
\hline BERRIGAN DR \& GREENBANK RD & 0.36 & 0.25 & 0.33 \\
\hline BLAIR RD \& BLAIR RD RAMP 36 & 0.55 & 1.00 & 0.67 \\
\hline BOOTH ST \& OTTAWA RIVER PKWY & 0.11 & 1.40 & 0.57 \\
\hline BRONSON AVE \& CARLING AVE & 2.00 & 2.92 & 2.86 \\
\hline BRONSON AVE \& COMMISSIONER ST & 0.91 & 0.75 & 0.87 \\
\hline CAMPEAU DR \& MARCH RD & 1.22 & 0.60 & 1.00 \\
\hline CARLING AVE \& ISLAND PARK DR & 0.33 & 1.40 & 0.71 \\
\hline CARLING AVE \& RICHMOND RD & 0.00 & 1.46 & 1.36 \\
\hline CATHERINE ST \& HWY 417 O'CONN & 2.22 & 1.80 & 2.07 \\
\hline CATHERINE ST \& KENT ST & 4.11 & 5.00 & 4.43 \\
\hline CHARLEMAGNE BLVD N \& TENTH LINE RD & 0.44 & 0.80 & 0.57 \\
\hline CONROY RD \& LORRY GREENBERG DR & 0.27 & 0.00 & 0.20 \\
\hline COVENTRY RD \& ST. LAURENT BLVD & 3.50 & 4.50 & 4.36 \\
\hline CYRVILLE RD \& INNES RD & 1.64 & 1.25 & 1.53 \\
\hline DRIVEWAY (THE) \& ISLAND PARK DR & 0.18 & 0.50 & 0.27 \\
\hline DUFORD DR \& ST. JOSEPH BLVD & 0.27 & 0.50 & 0.33 \\
\hline EAGLESON RD \& HAZELDEAN RD & 0.50 & 1.25 & 1.14 \\
\hline FISHER AVE \& MEADOWLANDS DR & 0.73 & 1.50 & 0.93 \\
\hline HAWTHORNE AVE \& QUEEN ELIZABETH DRWY & 0.36 & 0.25 & 0.33 \\
\hline HERON RD \& RIVERSIDE DR & 1.00 & 1.75 & 1.64 \\
\hline JOCKVALE RD \& STRANDHERD DR & 0.09 & 0.00 & 0.07 \\
\hline KING EDWARD AVE \& ST. ANDREW ST & 0.17 & 0.00 & 0.14 \\
\hline KING EDWARD AVE \& ST. PATRICK ST & 5.25 & 2.50 & 4.86 \\
\hline MEADOWLANDS DR \& MERIVALE RD & 1.00 & 2.00 & 1.86 \\
\hline RUSSELL RD \& WALKLEY RD & 2.33 & 5.60 & 3.50 \\
\hline SAUNDERSON DR \& SMYTH RD & 0.17 & 0.50 & 0.21 \\
\hline Grand Total & 1.42 & 1.96 & 1.58 \\
\hline
\end{tabular}




\section{Appendix B - Allocation of Costs}

\section{Adapted from : (Vodden et al., 2007)}

Total social cost by collision severity is: Fatal $-\$ 11.5$ billion; Injury $-\$ 5.0$ billion; and $\mathrm{PDO}-\$ 1.3$ billion. The average social cost by collision severity is: Fatal $-\$ 15.7$ million; Injury $-\$ 82$ thousand; and PDO — \$8 thousand. The average collision had a social cost of \$77 thousand in 2004.

Exhibit IV-1 Social Cost of motor vehicle collisions in Ontario in 2004 based on willingness to pay medium estimate (2004 million S)

Social costs of collisions in: 2004

Using values $(\$ 000,000)$ for: 2004

Valuation using: Medium estimate using Willingness to Pay

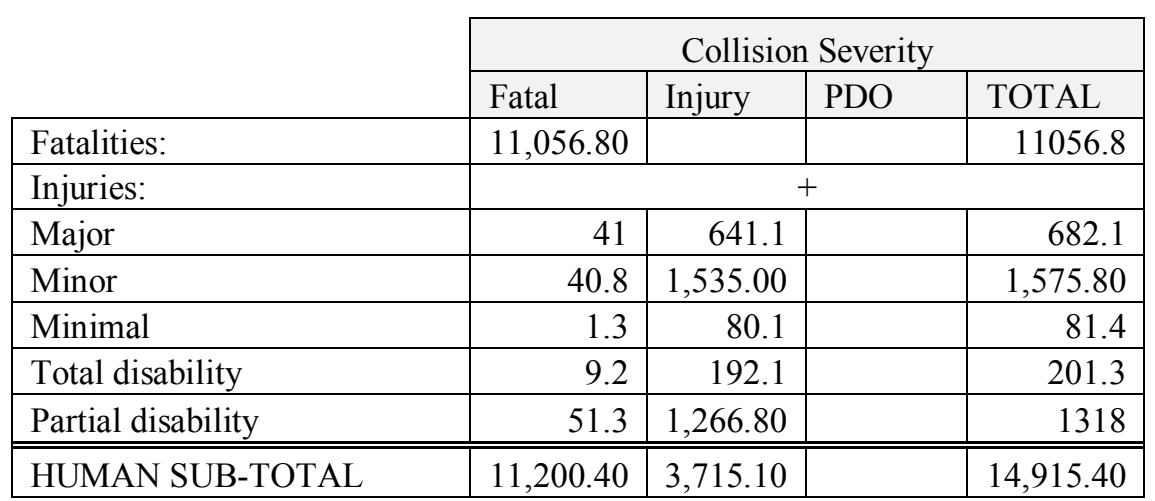

\begin{tabular}{|l|r|r|r|r|}
\hline Other Costs: & & & & \\
\hline Property damage & 17.5 & 740.9 & 1045.8 & 1804.2 \\
\hline Hospital/Health care: & & & & \\
\hline • ER facility & 0.2 & 6.5 & 0 & 6.8 \\
\hline • Hospital stay facility & 1.2 & 11 & & 12.1 \\
\hline • Medical practitioners & 4.3 & 99.7 & & 104 \\
\hline Police & 6.1 & 49.9 & 29.3 & 85.3 \\
\hline Courts & 0.9 & 7.3 & 4.3 & 12.6 \\
\hline Fire & 1.9 & 89.4 & & 91.3 \\
\hline Ambulance & 1.6 & 27.6 & & 29.1 \\
\hline Towtrucks & 0.6 & 33.2 & 61.8 & 95.6 \\
\hline Out of pocket & 0.6 & 56.3 & 149.3 & 206.2 \\
\hline Traffic delays: & & & & \\
\hline • Extra time & 120.6 & 102.2 & 27.9 & 250.7 \\
\hline • Extra fuel & 10.1 & 8.6 & 2.3 & 21 \\
\hline • Extra pollution & 110.7 & 93.8 & 25.6 & 230.2 \\
\hline \hline OTHER SUB-TOTAL & 276.30 & $1,326.40$ & $1,346.30$ & $2,949.10$ \\
\hline
\end{tabular}

\begin{tabular}{|l|r|r|r|r|}
\hline $\begin{array}{l}\text { OVERALL TOTAL } \\
\text { (human + other sub-totals) }\end{array}$ & $11,476.70$ & $5,041.50$ & $1,346.30$ & $17,864.50$ \\
\hline AVERAGE $(\$ 000)$ & $\mathbf{1 5 . 7 2 8}$ & $\mathbf{8 1 . 6}$ & $\mathbf{8}$ & $\mathbf{7 7 . 2}$ \\
\hline
\end{tabular}


Appendix C - Histogram of collision frequency by collision impact type 
RLC INTERSECTIONS (before and after periods)

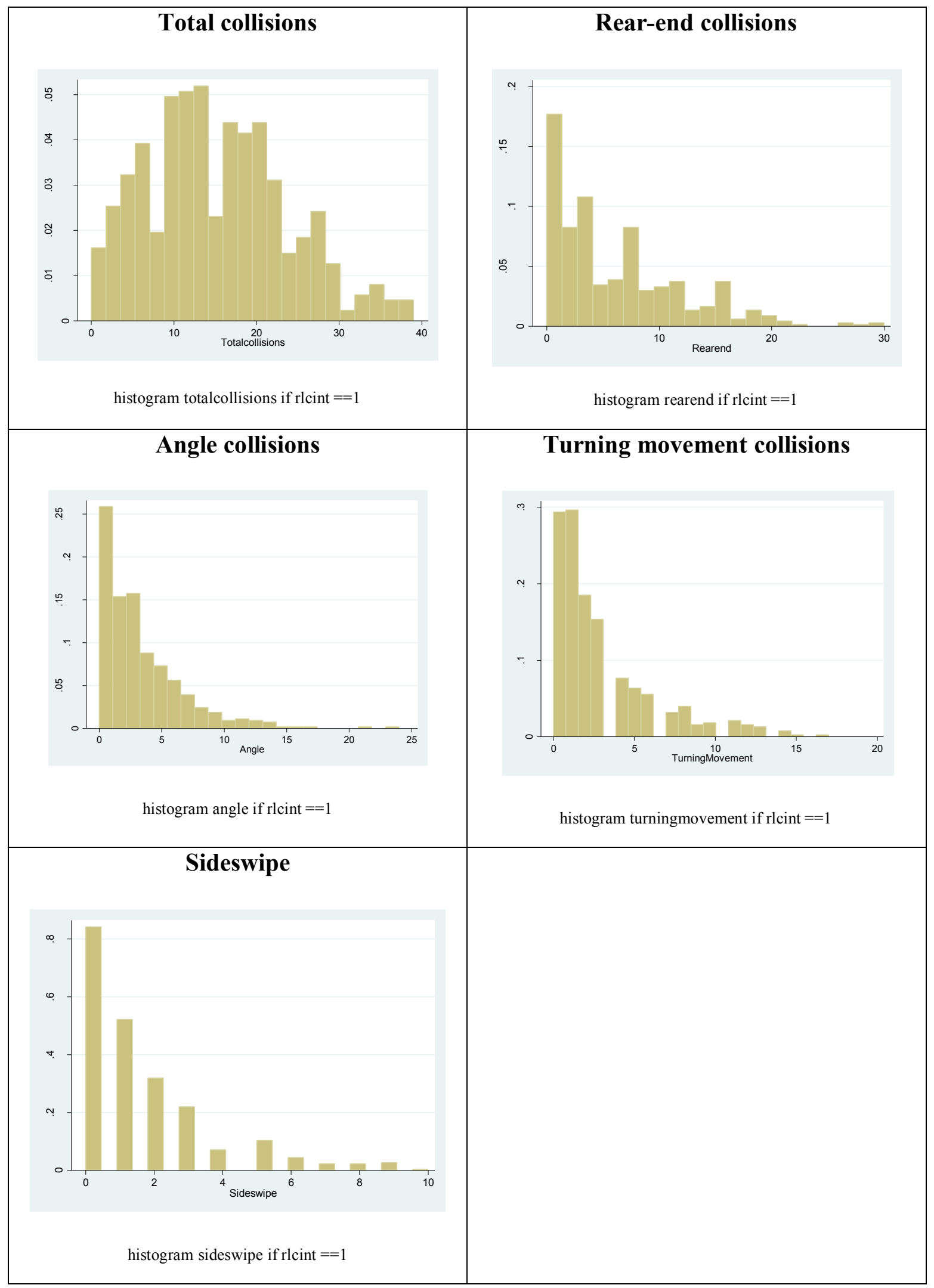




\section{RLC \& Non-RLC INTERSECTIONS (total collisions)}

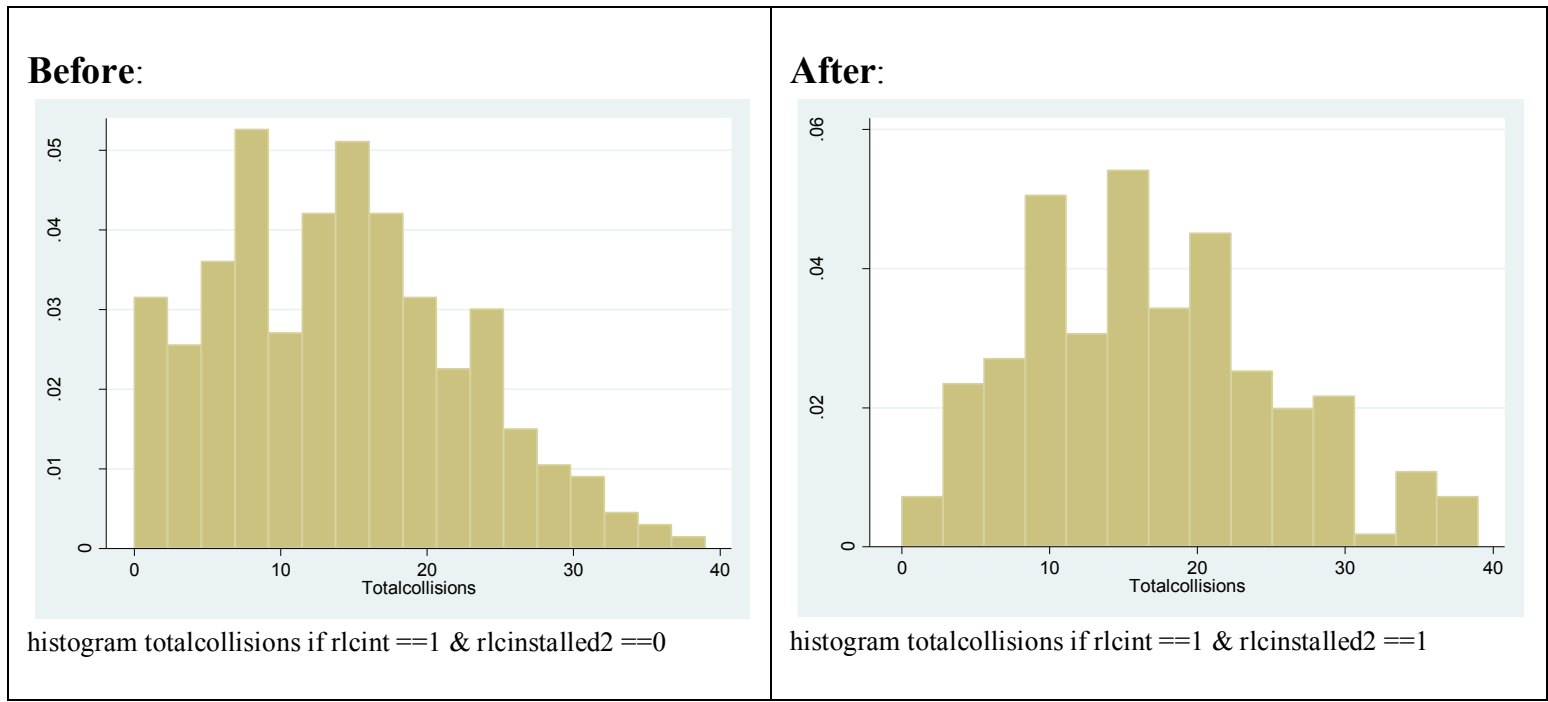

\section{Non-RLC INTERSECTIONS}

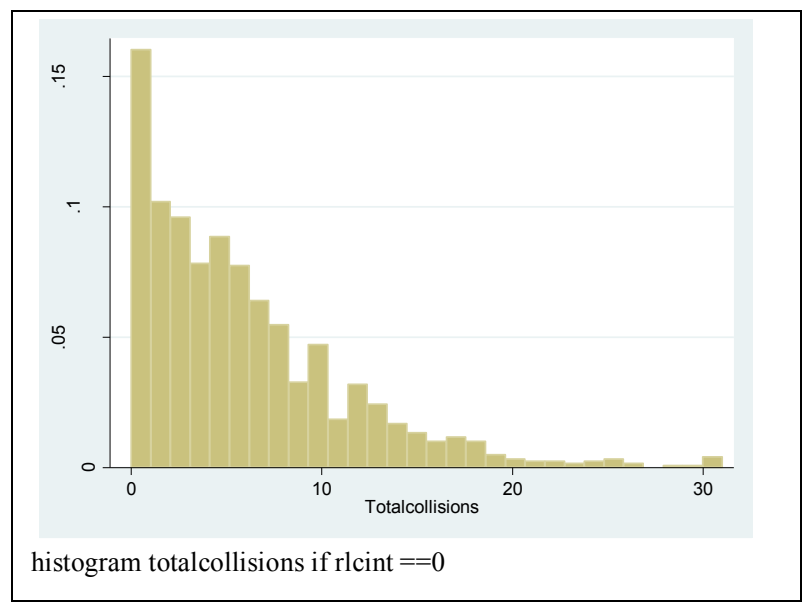




\section{Appendix D - Means and Variances}

. summarize totalcollisions rearend angle turningmovement sideswipe, detail

Totalcollisions

\begin{tabular}{|c|c|c|c|c|}
\hline & Percentiles & Smallest & & \\
\hline $1 \%$ & 1 & 0 & & \\
\hline $5 \%$ & 3 & 0 & & \\
\hline $10 \%$ & 5 & 0 & Obs & 685 \\
\hline $25 \%$ & 10 & 0 & Sum of Wgt. & 685 \\
\hline \multirow[t]{2}{*}{$50 \%$} & 14 & & Mean & $14.98832<$ \\
\hline & & Largest & Std. Dev. & 7.735381 \\
\hline $75 \%$ & 20 & 39 & & \\
\hline $90 \%$ & 26 & 39 & Variance & $59.83612^{<}$ \\
\hline $95 \%$ & 29 & 39 & Skewness & .5169208 \\
\hline $99 \%$ & 36 & 39 & Kurtosis & 3.058732 \\
\hline \multicolumn{5}{|c|}{ Rearend } \\
\hline & Percentiles & \multicolumn{3}{|c|}{---------------------------- - - - - - - - - - - - - - - - - - - - - - - - - - - } \\
\hline $1 \%$ & 0 & 0 & & \\
\hline $5 \%$ & 0 & 0 & & \\
\hline $10 \%$ & 1 & 0 & $\mathrm{Obs}$ & 685 \\
\hline $25 \%$ & 2 & 0 & Sum of Wgt. & 685 \\
\hline \multirow[t]{2}{*}{$50 \%$} & 5 & & Mean & $6.329927<$ \\
\hline & & Largest & Std. Dev. & 5.387531 \\
\hline $75 \%$ & 9 & 27 & & \\
\hline $90 \%$ & 14 & 28 & Variance & 29.02549 \\
\hline $95 \%$ & 16 & 30 & Skewness & 1.137234 \\
\hline \multirow[t]{2}{*}{$99 \%$} & 21 & 30 & Kurtosis & 4.386079 \\
\hline & \multicolumn{4}{|c|}{ Angle } \\
\hline & Percentiles & Smallest & & \\
\hline $1 \%$ & 0 & 0 & & \\
\hline $5 \%$ & 0 & 0 & & \\
\hline $10 \%$ & 0 & 0 & Obs & 685 \\
\hline $25 \%$ & 1 & 0 & Sum of Wgt. & 685 \\
\hline \multirow[t]{2}{*}{$50 \%$} & 3 & & Mean & 3.144526 \\
\hline & & Largest & Std. Dev. & 3.000169 \\
\hline $75 \%$ & 4 & 16 & & \\
\hline $90 \%$ & 7 & 17 & Variance & 9.001012 \\
\hline $95 \%$ & 9 & 21 & Skewness & 2.022987 \\
\hline \multirow[t]{2}{*}{$99 \%$} & 14 & 24 & Kurtosis & 9.907645 \\
\hline & \multicolumn{4}{|c|}{ TurningMovement } \\
\hline \multicolumn{5}{|c|}{--------------------------------------------------------------} \\
\hline & Percentiles & Smallest & & \\
\hline $1 \%$ & 0 & 0 & & \\
\hline $5 \%$ & 0 & 0 & & \\
\hline $10 \%$ & 0 & 0 & Obs & 685 \\
\hline $25 \%$ & 1 & 0 & Sum of Wgt. & 685 \\
\hline \multirow[t]{2}{*}{$50 \%$} & 2 & & Mean & 2.864234 \\
\hline & & Largest & Std. Dev. & 3.019521 \\
\hline $75 \%$ & 4 & 14 & & \\
\hline $90 \%$ & 7 & 14 & Variance & 9.117505 \\
\hline $95 \%$ & 9 & 15 & Skewness & 1.593698 \\
\hline $99 \%$ & 13 & 17 & Kurtosis & 5.654614 \\
\hline
\end{tabular}


Sideswipe

\begin{tabular}{|c|c|c|c|c|}
\hline & Percentiles & Smallest & & \\
\hline $1 \%$ & 0 & 0 & & \\
\hline $5 \%$ & 0 & 0 & & \\
\hline $10 \%$ & 0 & 0 & Obs & 685 \\
\hline $25 \%$ & 0 & 0 & Sum of Wgt. & 685 \\
\hline \multirow[t]{2}{*}{$50 \%$} & 1 & & Mean & 1.581022 \\
\hline & & Largest & Std. Dev. & 1.891329 \\
\hline $75 \%$ & 2 & 9 & & \\
\hline $90 \%$ & 4 & 9 & Variance & 3.577125 \\
\hline $95 \%$ & 5 & 9 & Skewness & 1.655795 \\
\hline $99 \%$ & 9 & 10 & Kurtosis & 5.958757 \\
\hline
\end{tabular}




\section{Appendix E - NB StataSE Output}

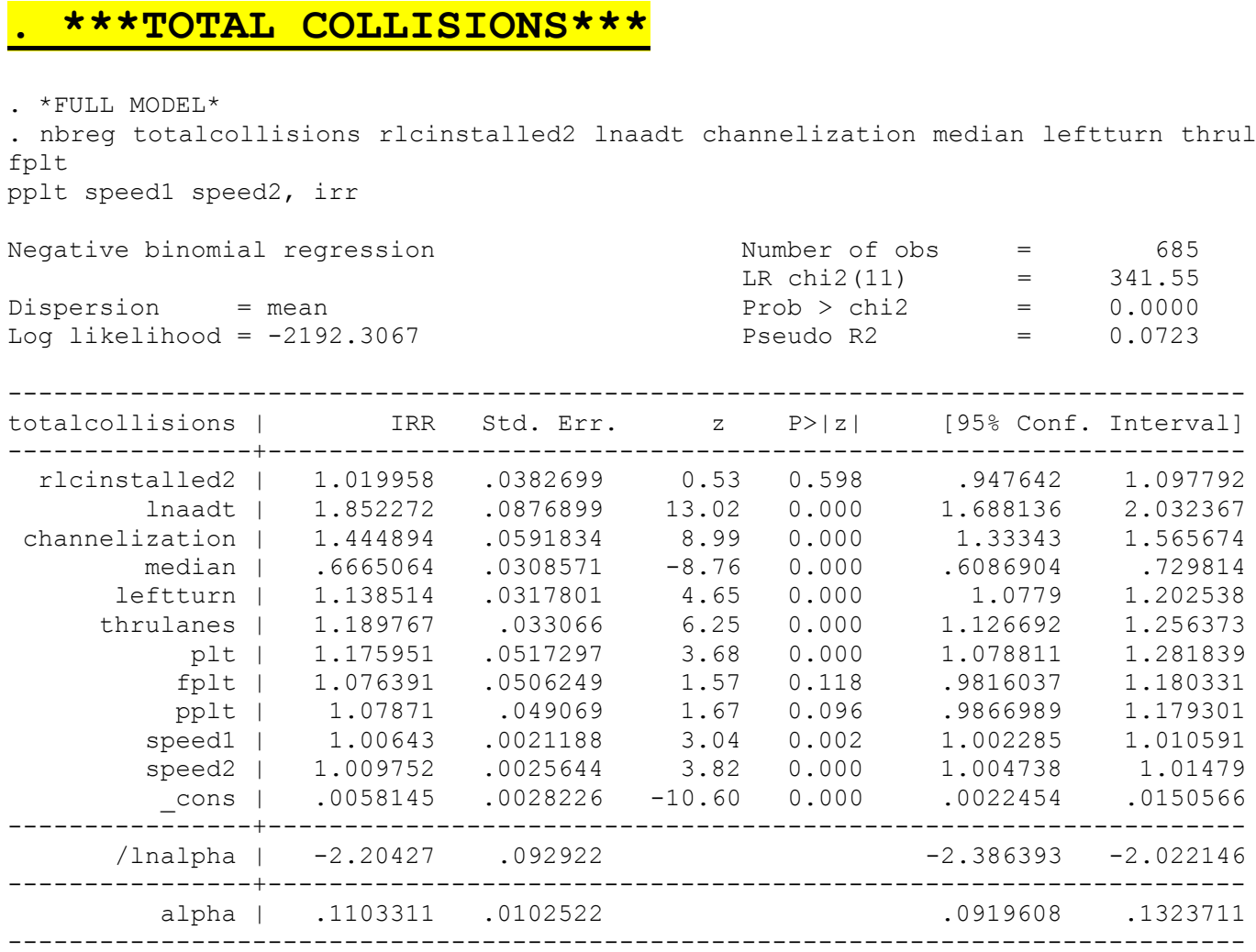

LR test of alpha=0: chibar2(01) $=407.85$

Prob $>=$ chibar2 $=0.000$

- fitstat

Measures of Fit for nbreg of totalcollisions

$\begin{array}{lrlr}\text { Log-Lik Intercept Only: } & -2363.083 & \text { Log-Lik Full Model: } & -2192.307 \\ \text { D(672): } & 4384.613 & \text { LR(11): } & 341.554 \\ & & \text { Prob > LR: } & 0.000 \\ \text { MCFadden's R2: } & 0.072 & \text { MCFadden's Adj R2: } & 0.067 \\ \text { Maximum Likelihood R2: } & 0.393 & \text { Cragg \& Uhler's R2: } & 0.393 \\ \text { AIC: } & 6.439 & \text { AIC*n: } & 4410.613 \\ \text { BIC: } & -3.156 & \text { BIC': } & -269.730\end{array}$

. *nbreg best fit reduced:

- nbreg totalcollisions rlcinstalled2 lnaadt channelization median leftturn thrulanes plt speed1 speed2, irr

Negative binomial regression

Dispersion= mean

Log likelihood $=-2194.0648$

Number of obs

LR chi2(9)

Prob > chi2

Pseudo R2

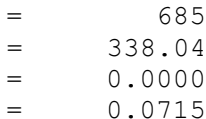

\begin{tabular}{|c|c|c|c|c|c|c|}
\hline totalcollisions & IRR & Std. Err. & z & $P>|z|$ & [95\% Conf. & Jal] \\
\hline lcinstalled2 & 1.009402 & .0375249 & 0.25 & 0.801 & .9384697 & 1.085695 \\
\hline lnaadt & 1.856836 & .0866584 & 13.26 & 0.000 & 1.694525 & 2.034693 \\
\hline rannelization & 1.436911 & .0586502 & 8.88 & 0.000 & 1.326436 & 1.556586 \\
\hline median & .6797706 & .0306337 & -8.57 & 0.000 & .6223049 & .7425429 \\
\hline leftturn & 1.14044 & .0318757 & 4.70 & 0.000 & 1.079645 & 1.204658 \\
\hline thrulanes & 1.17991 & .0322782 & 6.05 & 0.000 & 1.118312 & 1.244901 \\
\hline plt & 1.138942 & .0448 & 3.31 & 0.001 & 1.054435 & 1.230221 \\
\hline
\end{tabular}




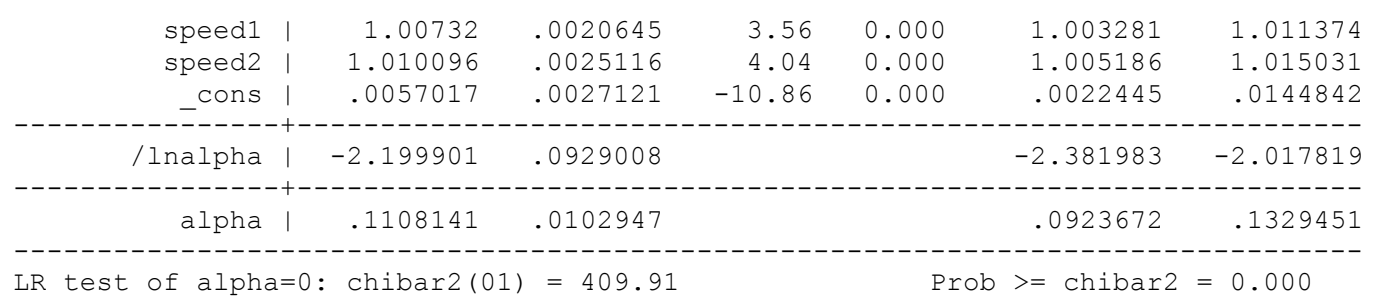

- fitstat

Measures of Fit for nbreg of totalcollisions

$\begin{array}{lrlr}\text { Log-Lik Intercept Only: } & -2363.083 & \text { Log-Lik Full Model: } & -2194.065 \\ \text { D(674): } & 4388.130 & \text { LR(9): } & 338.037 \\ & & \text { Prob > LR: } & 0.000 \\ \text { MCFadden's R2: } & 0.072 & \text { MCFadden's Adj R2: } & 0.067 \\ \text { Maximum Likelihood R2: } & 0.390 & \text { Cragg \& Uhler's R2: } & 0.390 \\ \text { AIC: } & 6.438 & \text { AIC*n: } & 4410.130 \\ \text { BIC: } & -12.699 & \text { BIC': } & \end{array}$

\section{. $* \star \star$ REAR-END COLLISIONS $* \star *$}

. * nbreg on full

- nbreg rearend rlcinstalled2 lnaadt channelization median leftturn thrulanes plt fplt pplt speed1 speed2, irr

Negative binomial regression

Dispersion $\quad=$ mean

Log likelihood $=-1745.095$

$\begin{array}{llr}\text { Number of obs } & = & 685 \\ \text { LR chi2(11) } & = & 476.06 \\ \text { Prob > chi2 } & = & 0.0000 \\ \text { Pseudo R2 } & = & 0.1200\end{array}$

\begin{tabular}{|c|c|c|c|c|c|c|}
\hline rearend | & IRR & Std. Err. & z & $P>|z|$ & [95\% Conf. & Interval] \\
\hline rlcinstalled2 | & 1.174396 & .0656937 & 2.87 & 0.004 & 1.052446 & 1.310476 \\
\hline Inaadt & 3.258538 & .2441723 & 15.76 & 0.000 & 2.813453 & 3.774035 \\
\hline nelization | & 1.492513 & .0913287 & 6.54 & 0.000 & 1.323829 & 1.68269 \\
\hline median | & .9892691 & .0716181 & -0.15 & 0.882 & .8584041 & 1.140085 \\
\hline leftturn | & 1.038635 & .0432963 & 0.91 & 0.363 & .9571501 & 1.127057 \\
\hline thrulanes | & 1.032596 & .0437878 & 0.76 & 0.449 & .9502436 & 1.122086 \\
\hline plt 1 & 1.050629 & .068674 & 0.76 & 0.450 & .9242958 & 1.19423 \\
\hline fplt & 1.312368 & .0912859 & 3.91 & 0.000 & 1.145111 & 1.504055 \\
\hline pplt I & 1.314319 & .0917481 & 3.92 & 0.000 & 1.146255 & 1.507024 \\
\hline speed1 | & 1.014974 & .0031003 & 4.87 & 0.000 & 1.008915 & 1.021068 \\
\hline speed2 | & 1.017641 & .0038088 & 4.67 & 0.000 & 1.010203 & 1.025133 \\
\hline cons I & $2.40 e-06$ & $1.84 e-06$ & -16.88 & 0.000 & $5.34 e-07$ & .0000108 \\
\hline /Inalpha | & -1.554048 & .108908 & & & -1.767503 & -1.340592 \\
\hline alpha | & .2113906 & .0230221 & & & .1707588 & .2616907 \\
\hline
\end{tabular}

LR test of alpha=0: chibar2(01) $=281.55$

Prob $>=$ chibar $2=0.000$

- fitstat

Measures of Fit for nbreg of rearend

Log-Lik Intercept Only: $\quad-1983.123$ D (672):

McFadden's R2:

Maximum Likelihood R2:

AIC :

BIC :

. *nbreg best fit reduced:
Log-Lik Full Model:

LR (11) :

Prob > LR:

0.120

0.501

5.133

$-897.580$

McFadden's Adj R2:

Cragg \& Uhler's R2:

$A I C^{\star} n$ :

BIC' :

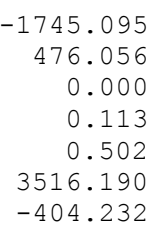

$-1745.095$

.000

0.113

$-404.232$ 
- nbreg rearend rlcinstalled2 lnaadt channelization fplt pplt speed1 speed2, irr Negative binomial regression

Number of obs LR Chi2(7) Prob > chi2 Dispersion $\quad=$ mean Log likelihood $=-1746.0532$
Pseudo R2

$\begin{array}{rrr}= & & 685 \\ = & & 474.14 \\ = & & 0.0000 \\ = & & 0.1195\end{array}$

rearend |
ren

LR test of alpha=0: $\operatorname{chibar2}(01)=292.35$

Prob $>=$ chibar $2=0.000$

- fitstat

Measures of Fit for nbreg of rearend

Log-Lik Intercept Only: $\quad-1983.123$

D (676):

3492.106

Log-Lik Full Model:

$-1746.053$

$\operatorname{LR}(7):$

474.139

Prob > LR:

0.000

MCFadden's Adj R2:

0.115

$\begin{array}{ll}\text { Maximum Likelihood R2: } & 0.500 \\ \text { AIC: } & 5.124\end{array}$

Cragg \& Uhler's R2:

0.501

$A I C^{\star} n$ :

3510.106

$-921.781$

$-428.434$

\section{. $* \star \star$ ANGLE COLLISIONS $* * *$}

. * nbreg on full model

- nbreg angle rlcinstalled2 lnaadt channelization median leftturn thrulanes plt fplt pplt speed1 speed2, irr

Negative binomial regression

Number of obs

LR $\operatorname{chi2}(11)$

Dispersion $\quad=$ mean

Log likelihood $=-1472.3882$

$\begin{array}{ll}\mathrm{chi2} & =0.0000\end{array}$

\begin{tabular}{|c|c|c|c|c|c|c|}
\hline angle | & IRR & Std. Err. & z & $P>|z|$ & [95\% Conf. & Interval] \\
\hline rlcinstalled2 | & .8239992 & .0594871 & -2.68 & 0.007 & .7152798 & .9492436 \\
\hline Inaadt & .8661252 & .0769089 & -1.62 & 0.106 & .7277747 & 1.030776 \\
\hline channelization & 1.085338 & .0856697 & 1.04 & 0.300 & .929772 & 1.266932 \\
\hline median & .7378556 & .0647453 & -3.46 & 0.001 & .6212697 & .8763197 \\
\hline leftturn & 1.261866 & .0683389 & 4.29 & 0.000 & 1.134788 & 1.403175 \\
\hline thrulanes | & 1.339935 & .071693 & 5.47 & 0.000 & 1.206536 & 1.488083 \\
\hline plt | & 1.013089 & .0895683 & 0.15 & 0.883 & .8519062 & 1.204767 \\
\hline fplt & .7142611 & .0657134 & -3.66 & 0.000 & .5964099 & .8553998 \\
\hline pplt i & .4934816 & .0448515 & -7.77 & 0.000 & .4129592 & .589705 \\
\hline speed1 & 1.001701 & .0044126 & 0.39 & 0.700 & .9930897 & 1.010387 \\
\hline speed2 | & .9978994 & .0049612 & -0.42 & 0.672 & .9882228 & 1.007671 \\
\hline _cons | & 9.977261 & 9.173026 & 2.50 & 0.012 & 1.645972 & 60.47839 \\
\hline /Inalpha | & -1.096737 & .1169352 & & & -1.325926 & -.8675482 \\
\hline alpha | & .333959 & .0390516 & & & .265557 & .41998 \\
\hline
\end{tabular}




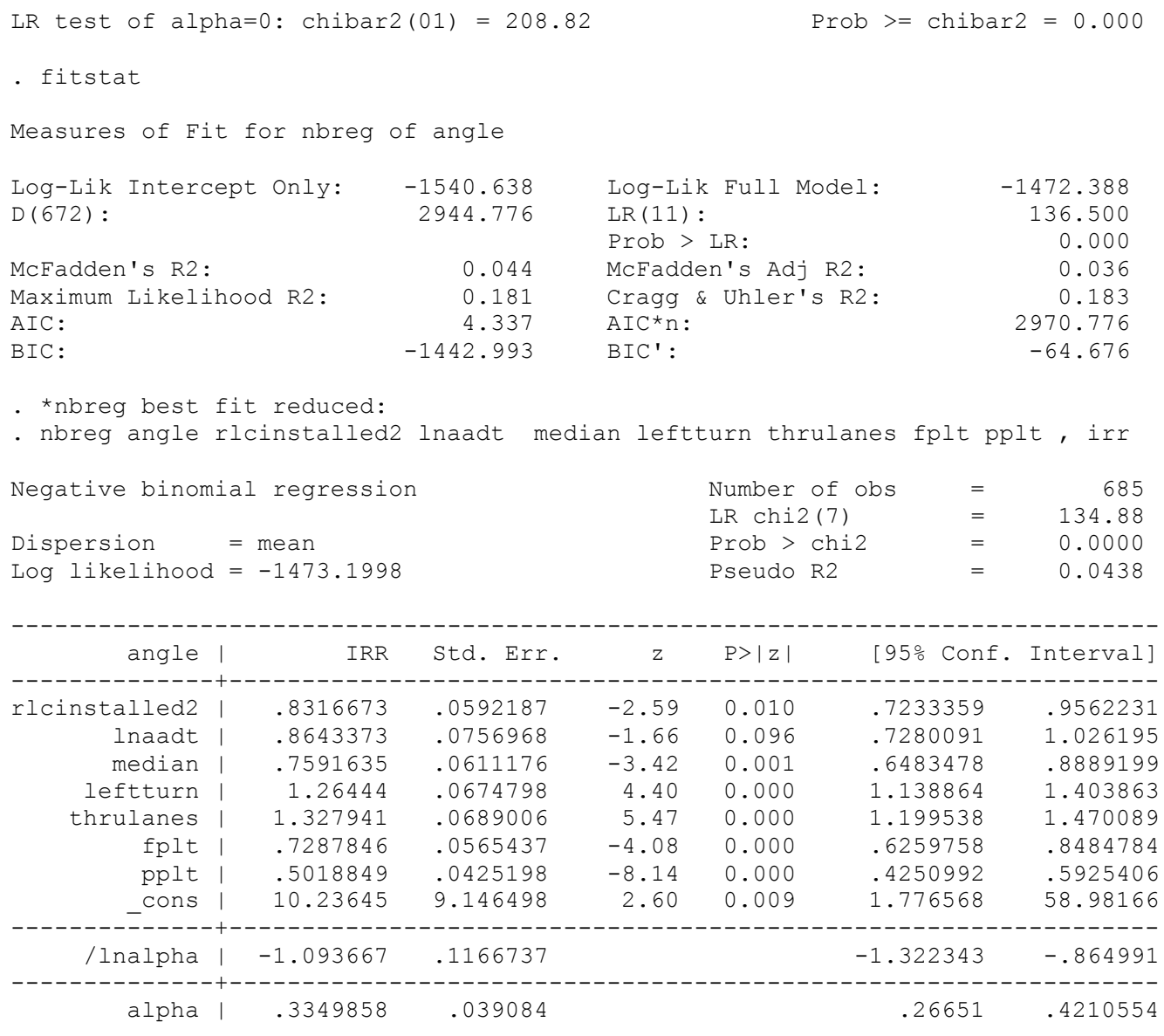

LR test of alpha=0: chibar2(01) $=211.45$

Prob $>=$ chibar $2=0.000$

- fitstat

Measures of Fit for nbreg of angle

Log-Lik Intercept Only: $\quad-1540.638$

D (676):

2946.400

Log-Lik Full Model:

$-1473.200$

LR (7):

134.877

Prob > LR:

0.000

MCFadden's R2:

0.044 McFadden's Adj R2:

Maximum Likelihood R2: $\quad 0.179$

AIC :

4.328

Cragg \& Uhler's R2:

0.038

$\mathrm{AIC}^{\star} \mathrm{n}$ :

0.181

$-1467.487$

BIC' :

2964.400

$-89.171$

\section{. $\star \star \star$ TURNINGMOVEMENT COLLISIONS ***}

. * nbreg on full model

- nbreg turningmovement rlcinstalled2 lnaadt channelization median leftturn thrulanes plt fplt pplt speed1 speed2, irr

$\begin{array}{lllr}\text { Negative binomial regression } & \text { Number of obs } & = & 685 \\ & \text { LR chi2 (11) } & = & 140.38 \\ \text { Dispersion }=\text { mean } & \text { Prob > chi2 } & = & 0.0000 \\ \text { Log likelihood }=-1437.7798 & \text { Pseudo R2 } & = & 0.0465\end{array}$

\begin{tabular}{|c|c|c|c|c|c|c|}
\hline turningmovement & IRR & Std. Err. & z & $\mathrm{P}>|\mathrm{z}|$ & [95\% Conf. & Interval] \\
\hline rlcinstalled2 & .8319201 & .0708887 & -2.16 & 0.031 & .7039632 & .9831353 \\
\hline lnaadt & 1.597505 & .1756352 & 4.26 & 0.000 & 1.287829 & 1.981647 \\
\hline channelization & 1.774666 & .1647146 & 6.18 & 0.000 & 1.479493 & 2.128729 \\
\hline median & .4363698 & .0446738 & -8.10 & 0.000 & .3570361 & .5333316 \\
\hline
\end{tabular}




\begin{tabular}{|c|c|c|c|c|c|c|}
\hline leftturn & .9232845 & .0606008 & -1.22 & 0.224 & .8118317 & 1.050038 \\
\hline thrulanes | & 1.116897 & .0673373 & 1.83 & 0.067 & .9924175 & 1.25699 \\
\hline plt | & 1.261375 & .121923 & 2.40 & 0.016 & 1.043682 & 1.524475 \\
\hline fplt & .9388935 & .098942 & -0.60 & 0.550 & .763687 & 1.154296 \\
\hline pplt | & 1.93258 & .1871464 & 6.80 & 0.000 & 1.598487 & 2.3365 \\
\hline speed1 & .9934389 & .0045911 & -1.42 & 0.154 & .9844811 & 1.002478 \\
\hline speed2 | & 1.005566 & .0058379 & 0.96 & 0.339 & .9941889 & 1.017074 \\
\hline _cons I & .0189012 & .021111 & -3.55 & 0.000 & .0021172 & .1687366 \\
\hline /Inalpha | & -.6951847 & .105285 & & & -.9015395 & -.488829 \\
\hline alpha & .4989823 & .0525354 & & & .4059442 & .613343 \\
\hline
\end{tabular}

LR test of alpha $=0$ : chibar2(01) $=302.29$

Prob $>=$ chibar2 $=0.000$

- fitstat

Measures of Fit for nbreg of turningmovement

Log-Lik Intercept Only: $\quad-1507.971$

D (672):

2875.560

Log-Lik Full Model:

$-1437.780$

$\mathrm{LR}(11)$ :

140.382

Prob > LR:

0.000

MCFadden's R2:

0.047 McFadden's Adj R2:

0.038

Maximum Likelihood R2:

0.185

Cragg \& Uhler's R2:

0.188

AIC :

4.236

$A I C^{\star} \mathrm{n}$ :

2901.560

BIC :

$-1512.210$

BIC' :

$-68.559$

: nbreg turningmovement rlcinstalled2 lnaadt channelization median thrulanes plt pplt, irr

Negative binomial regression

Number of obs

LR chi2(7)

Prob > chi2

Pseudo R2

$\begin{array}{rr}= & 685 \\ = & 135.20 \\ = & 0.0000 \\ = & 0.0448\end{array}$

$\begin{array}{ll}\text { Log likelihood } & =-1440.3706\end{array}$

IRR Std. Err.

$\mathrm{z} \quad \mathrm{P}>|\mathrm{z}|$

[95\% Conf. Interval]

turningmovement

$-2.13$

0.033

0.000

$.7096176 \quad .9861147$

rlcinstalled2 | lnaadt

1.587854

.0702191

4.37

0.000

1.290327

1.953987

channelization

1.741691

.1598015

6.05

0.000

1.455033

2.084825

thrulanes

1.144794

.0412719

2.35

0.019

.3530639

.5158172

plt | 1.318148

.1146117

3.18

0.001

1.022633

1.281547

pplt | 1.925666

.1562743

8.07

0.000

1.563059

_cons I .0167683

.0183003

$-3.75$

0.000

.0019748

2.257661

/lnalpha | -.6739772 .1038748

$-.877568-.4703863$

alpha

.5096775

.0529426

.4157929

.6247609

LR test of alpha=0: chibar2(01) $=317.09$

Prob $>=$ chibar2 $=0.000$

- fitstat

Measures of Fit for nbreg of turningmovement

Log-Lik Intercept Only:

D (676):

McFadden's R2:

Maximum Likelihood R2:

AIC :

BIC :

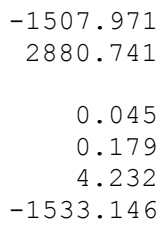

$-1507.971$

2880.741

0.045

0.179

4.232

$-1533.146$

Log-Lik Full Model:

$\operatorname{LR}(7)$ :

Prob > LR:

McFadden's Adj R2:

Cragg \& Uhler's R2:

$A I C^{\star} n$ :

BIC' :

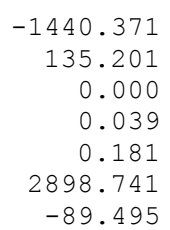

. $* \star \star$ SIDESWIPE COLIISIONS $* \star *$ 
. * nbreg on full model

- nbreg sideswipe rlcinstalled2 lnaadt channelization median leftturn thrulanes plt fplt pplt speed1 speed2, irr

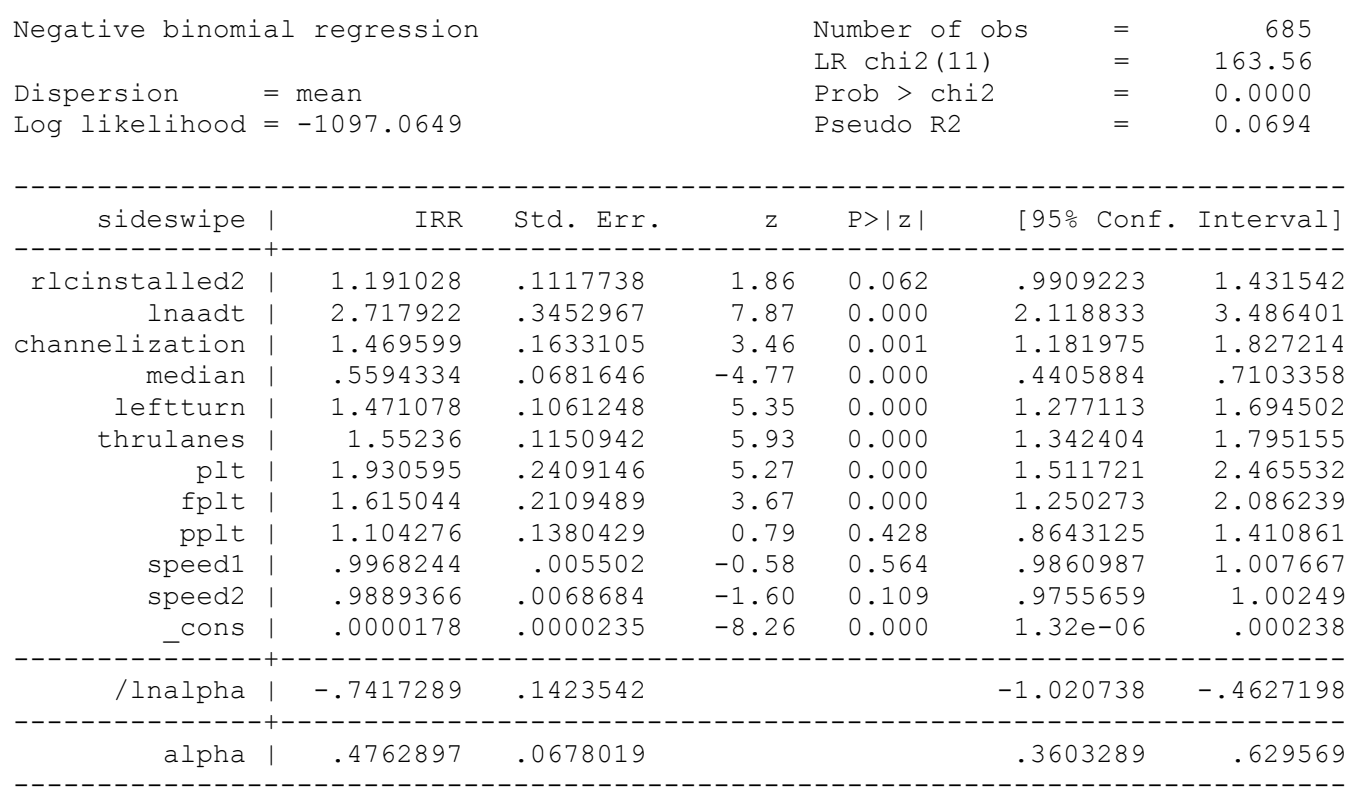

LR test of alpha $=0$ : chibar2 $(01)=124.88$

Prob $>=$ chibar $2=0.000$

- fitstat

Measures of Fit for nbreg of sideswipe

Log-Lik Intercept Only: $\quad-1178.846$

D (672):

2194.130

Log-Lik Full Model:

McFadden's R2:

Maximum Likelihood R2:

AIC :

BIC :

* *nbreg best fit reduced:

- nbreg sideswipe rlcinstalled2 lnaadt channelization median leftturn thrulanes plt fplt, irr

Negative binomial regression

Dispersion = mean

Log likelihood $=-1098.751$
Number of obs LR chi2(8)

Prob > chi2

Pseudo R2
LR (11):

Prob > LR:

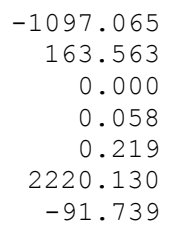

$-1097.065$

0.000

0.058

Cragg \& Uhler's R2:

$A I C^{*} \mathrm{n}$ :

BIC' :
220.130

$-91.739$

\begin{tabular}{|c|c|c|c|c|c|c|}
\hline sideswipe & IRR & Std. Err. & z & $P>|z|$ & [95\% Conf. & Interval] \\
\hline rlcinstalled2 & 1.185355 & .1104918 & 1.82 & 0.068 & .9874258 & 1.422959 \\
\hline Inaadt & 2.613627 & .3260831 & 7.70 & 0.000 & 2.046659 & 3.337657 \\
\hline channelization & 1.44609 & .1600335 & 3.33 & 0.001 & 1.164115 & 1.796365 \\
\hline median & .5718935 & .0668041 & -4.78 & 0.000 & .4548672 & .719028 \\
\hline leftturn & 1.468092 & .1055245 & 5.34 & 0.000 & 1.275176 & 1.690194 \\
\hline thrulanes & 1.501616 & .1068001 & 5.72 & 0.000 & 1.306227 & 1.726233 \\
\hline plt & 1.855699 & .219677 & 5.22 & 0.000 & 1.47144 & 2.340305 \\
\hline fplt & 1.468589 & .1616191 & 3.49 & 0.000 & 1.183655 & 1.822114 \\
\hline _cons & .0000142 & .0000185 & -8.62 & 0.000 & $1.13 e-06$ & .0001804 \\
\hline /lnalpha & -.7254988 & .1408413 & & & -1.001543 & -.449455 \\
\hline alpha & .484083 & .0681789 & & & .3673123 & .6379758 \\
\hline
\end{tabular}

LR test of alpha=0: chibar2(01) $=129.19$

Prob $>=$ chibar $2=0.000$ 


\section{- fitstat}

Measures of Fit for nbreg of sideswipe

Log-Lik Intercept Only:

D (675):

McFadden's R2:

Maximum Likelihood R2:

AIC:

BIC :

$\begin{aligned}-1178.846 & \text { Log-Lik Full Model: } \\ 2197.502 & \text { LR(8): } \\ & \text { Prob > LR: } \\ 0.068 & \text { MCFadden's Adj R2: } \\ 0.209 & \text { Cragg \& Uhler's R2: } \\ 3.237 & \text { AIC*n: } \\ -2209.856 & \text { BIC': }\end{aligned}$

$-1178.846$
$-1098.751$

160.191

0.000

0.059

0.215

2217.502

$-107.956$ 


\section{Appendix F - FE vs. RE StataSE Output}

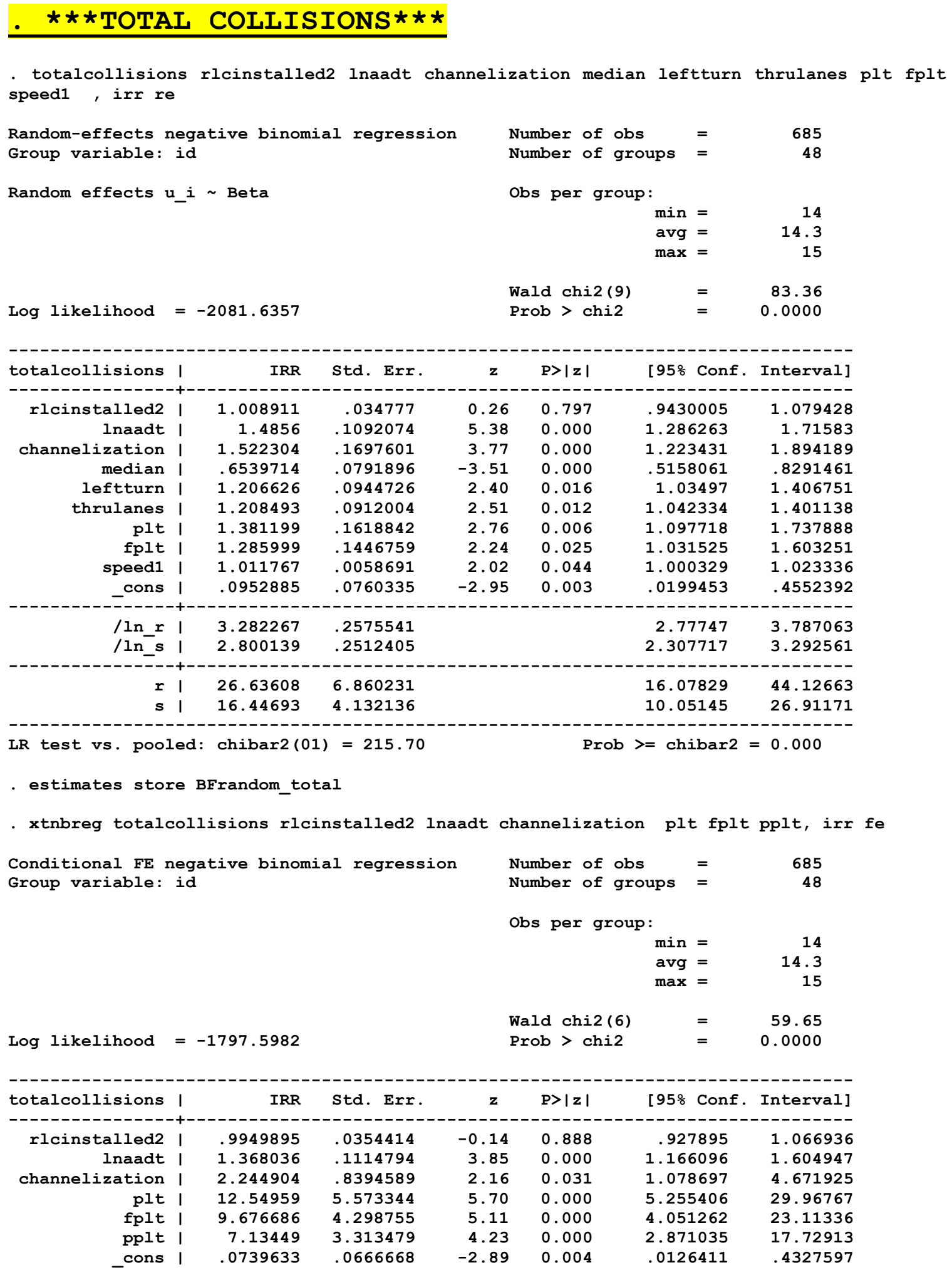


- estimates store BFfixed_total
. hausman BFfixed_total BFrandom_total

---- Coefficients ----

\begin{tabular}{|c|c|c|c|c|c|}
\hline & 1 & $\begin{array}{c}\text { (b) } \\
\text { BFfixed_to l }\end{array}$ & $\begin{array}{c}\text { (B) } \\
\text { BFrandom_t l }\end{array}$ & $\begin{array}{c}(b-B) \\
\text { Difference }\end{array}$ & $\begin{array}{c}\left.\operatorname{sqrt}\left(\operatorname{diag}_{(\mathrm{V}} \mathrm{V}_{-} \mathrm{b}_{-} \mathrm{V}_{-} \mathrm{B}\right)\right) \\
\text { S.E. }\end{array}$ \\
\hline rlcinstall 2 & I & -.005023 & .0088713 & -.0138943 & .0089777 \\
\hline Inaadt & i & .3133759 & .395819 & -.0824431 & .0351653 \\
\hline channeliza n & $i$ & .8086627 & .4202247 & .3884379 & .3569248 \\
\hline plt & i & 2.529688 & .322952 & 2.206736 & .4283604 \\
\hline fplt & i & 2.26972 & .2515359 & 2.018184 & .4297573 \\
\hline
\end{tabular}

$\mathrm{b}=$ consistent under $\mathrm{Ho}$ and $\mathrm{Ha}$; obtained from xtnbreg $\mathrm{B}=$ inconsistent under $\mathrm{Ha}$, efficient under Ho; obtained from xtnbreg

Test: Ho: difference in coefficients not systematic

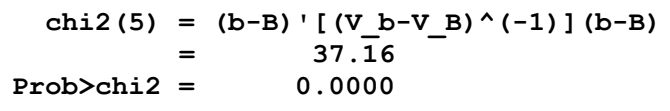

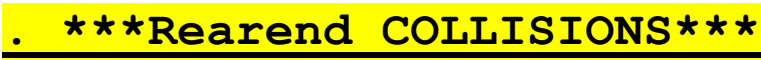

- xtnbreg rearend rlcinstalled2 lnaadt channelization fplt pplt speed2, irr re

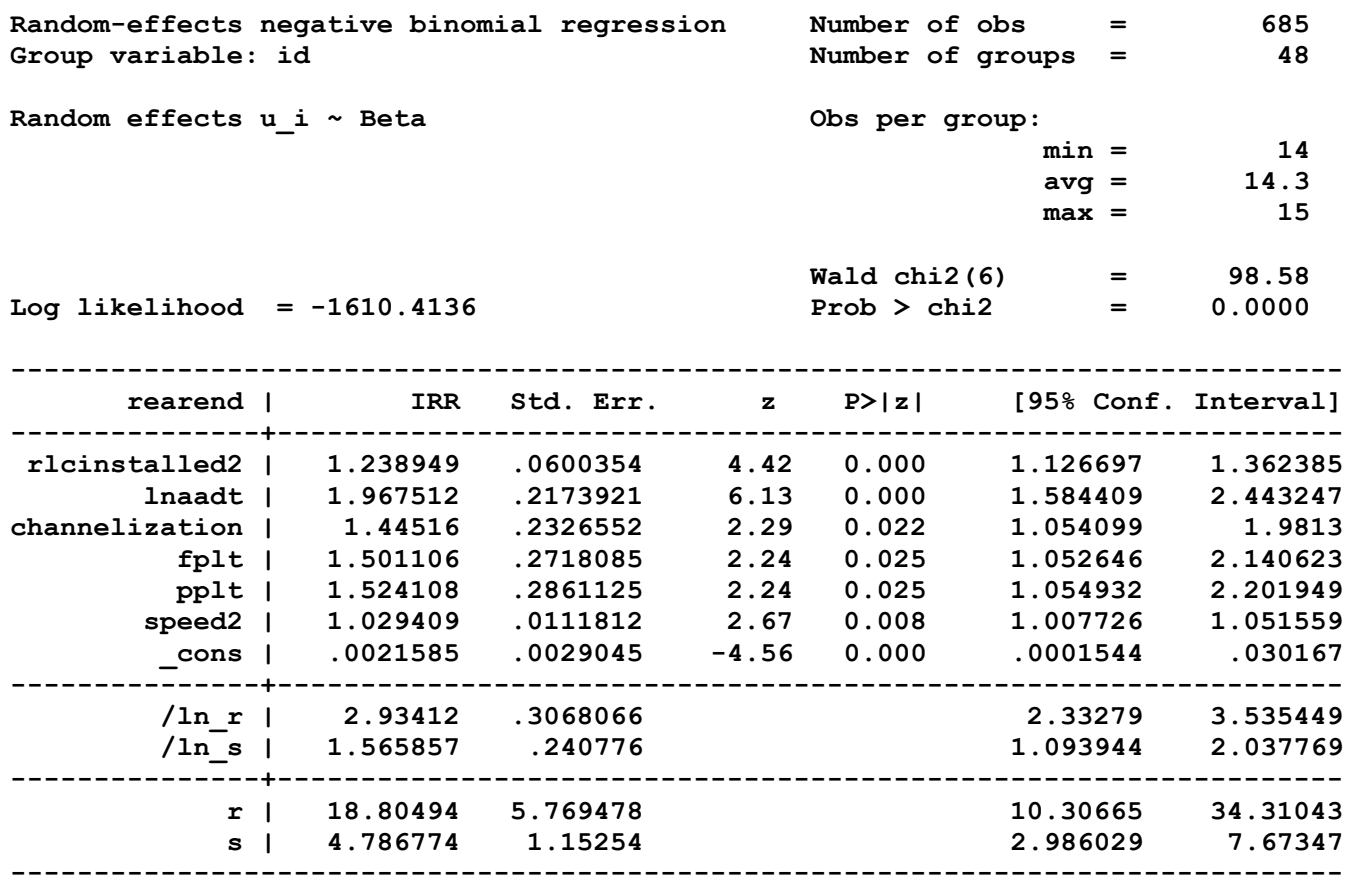

LR test vs. pooled: chibar2 $(01)=259.83 \quad$ Prob $>=$ chibar2 $=0.000$

- estimates store BFrandom_Rear

- xtnbreg rearend rlcinstalled2 lnaadt channelization median leftturn thrulanes plt fplt pplt speed1 speed2, irr fe

Conditional FE negative binomial regression Group variable: id

$\begin{array}{rrr}\text { Number of obs } & = \\ \text { Number of groups } & = & 685 \\ \text { Obs per group: } & 48 \\ \min & = & \\ \operatorname{avg} & = & 14.3 \\ \max & = & 15\end{array}$




\begin{tabular}{|c|c|c|c|c|c|c|c|}
\hline Log likelihood & $=$ & -1361.5256 & & & $\begin{array}{l}\text { ald } \mathrm{ch} \\
\text { rob }>\end{array}$ & $\begin{array}{l}= \\
=\end{array}$ & $\begin{array}{r}49.71 \\
0.0000\end{array}$ \\
\hline rearend & 1 & IRR & Std. Err. & $\mathbf{z}$ & $P>|z|$ & [95\% Conf. & Interval] \\
\hline $\begin{array}{r}\text { rlcinstalled2 } \\
\text { lnaadt }\end{array}$ & I & $\begin{array}{l}1.234684 \\
1.871255\end{array}$ & $\begin{array}{r}.06217 \\
.2504416\end{array}$ & $\begin{array}{l}4.19 \\
4.68\end{array}$ & $\begin{array}{l}0.000 \\
0.000\end{array}$ & $\begin{array}{r}1.118653 \\
1.4395\end{array}$ & $\begin{array}{r}1.362751 \\
2.43251\end{array}$ \\
\hline channelization & I & 2.035007 & 1.866155 & 0.77 & 0.438 & .3372811 & 12.27835 \\
\hline median & 1 & 1.372049 & 1.698674 & 0.26 & 0.798 & .1212087 & 15.5312 \\
\hline leftturn & i & .7384626 & .4241543 & -0.53 & 0.598 & .239563 & 2.27634 \\
\hline thrulanes & i & .2633071 & .1928093 & -1.82 & 0.068 & .0626847 & 1.106022 \\
\hline plt & 1 & 17.18604 & 35.49233 & 1.38 & 0.168 & .3001156 & 984.1544 \\
\hline fplt & i & 1.093169 & 1.416797 & 0.07 & 0.945 & .0861966 & 13.86386 \\
\hline pplt & 1 & .8136977 & 1.332419 & -0.13 & 0.900 & .0328573 & 20.15087 \\
\hline speed1 & i & .9938791 & .0371239 & -0.16 & 0.869 & .9237172 & 1.06937 \\
\hline speed2 & i & 1.096884 & .0876635 & 1.16 & 0.247 & .9378473 & 1.282889 \\
\hline cons & i & .0033124 & .0129585 & -1.46 & 0.144 & $1.55 e-06$ & 7.081227 \\
\hline
\end{tabular}

- estimates store fixed_rearend
. hausman fixed_rearend BFrandom_Rear

---- Coefficients ----

\begin{tabular}{|c|c|c|c|c|c|}
\hline & 1 & (b) & (B) & $(b-B)$ & sqrt (diag (V_b-V_B)) \\
\hline & I & fixed_rear d & BFrandom_R r & Difference & S.E. \\
\hline---------1 & + & ------- & ----------1 & ----- & ---- \\
\hline rlcinstall 2 & I & .2108151 & .2142637 & -.0034486 & .0136884 \\
\hline lnaadt & I & .6266096 & .6767696 & -.05016 & .0755239 \\
\hline channeliza n & i & .7104995 & .3682199 & .3422796 & .9027841 \\
\hline fplt & i & .0890804 & .406202 & -.3171215 & 1.283335 \\
\hline pplt & i & -.2061664 & .4214094 & -.6275759 & 1.62669 \\
\hline speed2 & I & .0924732 & .028985 & .0634882 & .079179 \\
\hline
\end{tabular}

$\mathrm{b}=$ consistent under $\mathrm{Ho}$ and $\mathrm{Ha}$; obtained from xtnbreg B = inconsistent under $\mathrm{Ha}$, efficient under Ho; obtained from xtnbreg

Test: Ho: difference in coefficients not systematic

$$
\begin{array}{rlrl}
\operatorname{chi2}(6) & = & (b-B) \cdot\left[\left(V_{-} b-V_{B} B\right)^{\wedge}(-1)\right](b-B) \\
& = & 2.41 \\
\text { Prob }>\operatorname{chi2} & = & & 0.8780
\end{array}
$$

\section{. $\star \star \star A$ ANGLE COLLISIONS $* * *$}

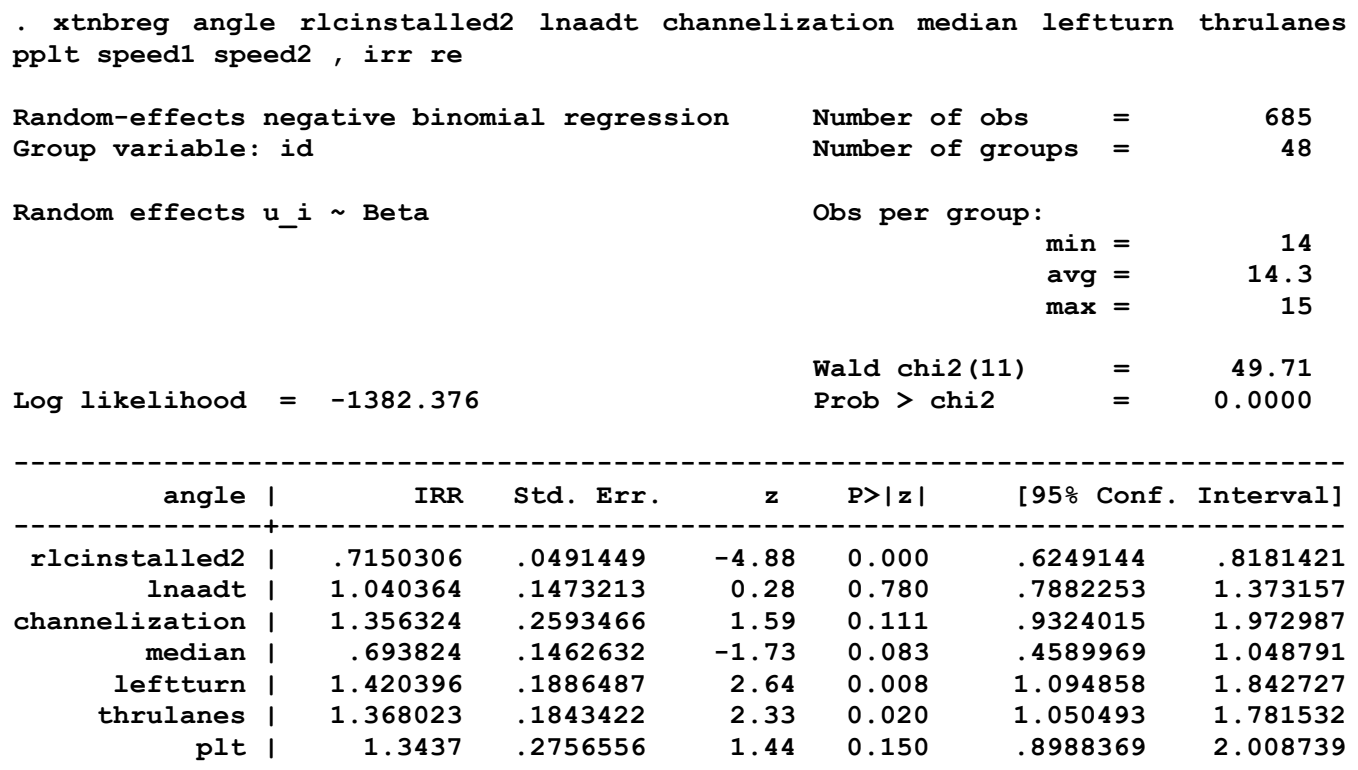




\begin{tabular}{|c|c|c|c|c|c|c|}
\hline fplt I & .9459641 & .2028186 & -0.26 & 0.796 & .6214034 & 1.440044 \\
\hline pplt I & .5628259 & .1162595 & -2.78 & 0.005 & .3754452 & 8437261 \\
\hline speed1 | & 1.008716 & .0109008 & 0.80 & 0.422 & .9875759 & 1.030309 \\
\hline speed2 | & .992286 & .0120294 & -0.64 & 0.523 & .9689866 & 1.016146 \\
\hline _cons I & 2.556821 & 4.07437 & 0.59 & 0.556 & .1125344 & 58.09188 \\
\hline lin_r & 2.636994 & .2944946 & & & 2.059795 & 3.214193 \\
\hline /ln_s I & 1.563296 & .2534216 & & & 1.066599 & 2.059993 \\
\hline $\begin{array}{ll}r & 1 \\
s & 1\end{array}$ & $\begin{array}{l}13.97114 \\
4.774531\end{array}$ & $\begin{array}{l}4.114425 \\
1.209969\end{array}$ & & & $\begin{array}{r}7.844362 \\
2.90548\end{array}$ & $\begin{array}{r}24.88319 \\
7.845915\end{array}$ \\
\hline
\end{tabular}

LR test vs. pooled: chibar2(01) $=177.61$

Prob $>=$ chibar2 $=0.000$.

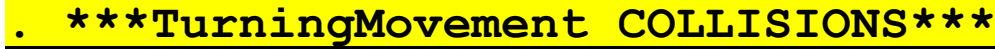

- xtnbreg turningmovement rlcinstalled2 lnaadt channelization median leftturn thrulanes plt fplt pplt speed1 speed2, irr re

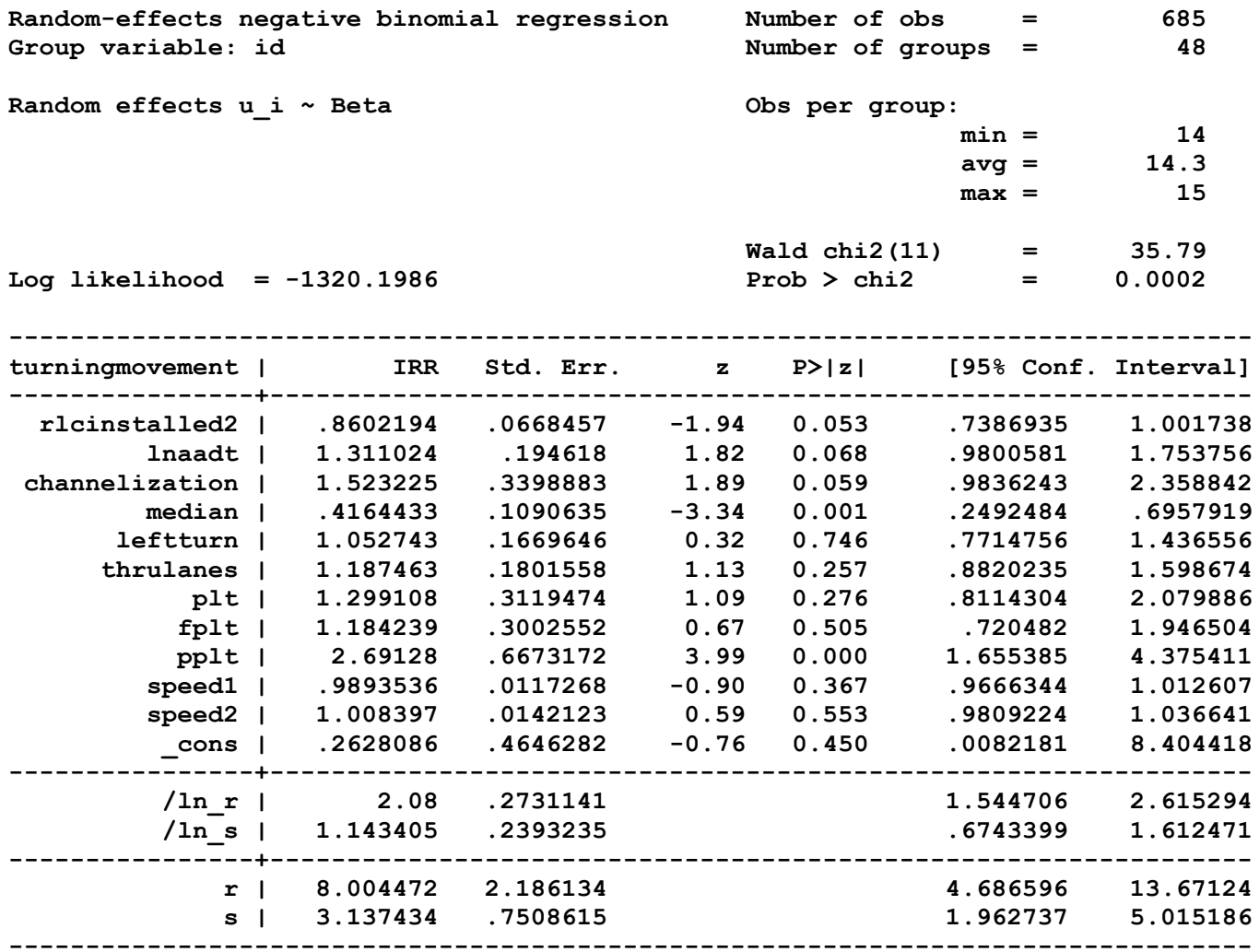

LR test vs. pooled: chibar2(01) $=221.58$

Prob $>=$ chibar $2=0.000$

- estimates store random_turningmovement

- xtnbreg turningmovement rlcinstalled2 lnaadt channelization median leftturn thrulanes plt fplt pplt speed1 speed2, irr fe

Conditional $\mathrm{FE}$ negative binomial regression Group variable: id

Log likelihood $=-1098.2685$

$\begin{array}{rrr}\text { Number of obs } & = \\ \text { Number of groups } & = & 685 \\ \text { Obs per group: } & \\ \min & = & 48 \\ \text { avg } & = & 14.3 \\ \max & = & 15 \\ \text { Wald chi2(11) } & = & 27.25 \\ \text { Prob }>\text { chi2 } & = & 0.0042\end{array}$




IRR
turningmovement |

estimates store fixed_turningmovement

. hausman fixed_turningmovement random_turningmovement

\begin{tabular}{|c|c|c|c|c|c|}
\hline & 1 & $\begin{array}{l}\text { (b) } \\
\text { fixed_turn t }\end{array}$ & $\begin{array}{l}\text { (B) } \\
\text { random_tur t }\end{array}$ & $\begin{array}{c}(b-B) \\
\text { Difference }\end{array}$ & $\begin{array}{c}\operatorname{sqrt}\left(\text { diag }\left(V_{-} b-V_{-} B\right)\right) \\
\text { S.E. }\end{array}$ \\
\hline rlcinstall 2 & I & -.1452014 & -.1505678 & .0053664 & .0067675 \\
\hline lnaadt & i & .2128124 & .2708081 & -.0579958 & .0623007 \\
\hline channeliza n & 1 & .015535 & .4208298 & -.4052948 & .3514847 \\
\hline median & i & -1.336741 & -.876005 & -.4607356 & .5764989 \\
\hline leftturn & 1 & .323188 & .0513992 & .2717887 & .2665104 \\
\hline thrulanes & 1 & .5754285 & .1718191 & .4036094 & .3169492 \\
\hline plt & i & .3140063 & .2616782 & .0523281 & .478947 \\
\hline fplt & i & .5692068 & .1691 & .4001068 & 4113273 \\
\hline pplt & 1 & 2.039919 & .9900171 & 1.049902 & .573239 \\
\hline speed1 & i & -.0380625 & -.0107035 & -.027359 & .0245242 \\
\hline speed2 & i & -.0070192 & .0083616 & -.0153809 & .0224361 \\
\hline
\end{tabular}

$\mathrm{b}=$ consistent under $\mathrm{Ho}$ and $\mathrm{Ha}$; obtained from $\mathrm{xtnbreg}$ $\mathrm{B}=$ inconsistent under $\mathrm{Ha}$, efficient under Ho; obtained from xtnbreg

Test: Ho: difference in coefficients not systematic

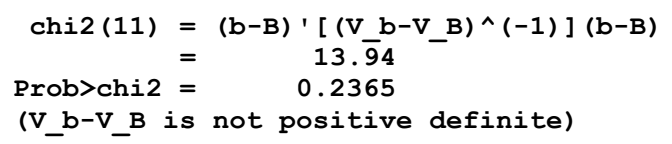

\section{. $* \star \star$ Sideswipe COLLISIONS $* \star *$}

- xtnbreg sideswipe rlcinstalled2 lnaadt channelization median leftturn thrulanes plt fplt, irr re

Random-effects negative binomial regression Group variable: id

Random effects $u_{-} i \sim$ Beta

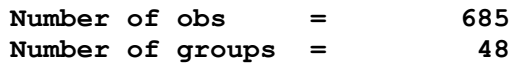

Obs per group:

$\begin{array}{rlr}\min & = & 14 \\ \operatorname{avg}= & & 14.3 \\ \max & = & \\ & \\ = & & 45.22 \\ & = & 0.0000\end{array}$

\begin{tabular}{|c|c|c|c|c|c|c|}
\hline sideswipe & IRR & Std. Err. & $\mathbf{z}$ & $P>|z|$ & [95\% Conf. & Interval] \\
\hline rlcinstalled2 | & 1.344971 & .1183631 & 3.37 & 0.001 & 1.131889 & 1.598167 \\
\hline lnaadt & 1.835545 & .3519391 & 3.17 & 0.002 & 1.260548 & 2.672825 \\
\hline channelization | & 1.503055 & .3761641 & 1.63 & 0.103 & .9203387 & 2.45472 \\
\hline
\end{tabular}




\begin{tabular}{|c|c|c|c|c|c|c|}
\hline median I & .5479913 & .1405835 & -2.34 & 0.019 & .3314391 & .9060323 \\
\hline leftturn $\mid$ & 1.415549 & .2353462 & 2.09 & 0.037 & 1.021894 & 1.960848 \\
\hline thrulanes & 1.50017 & .2471232 & 2.46 & 0.014 & 1.08623 & 2.0718 \\
\hline plt I & 1.833879 & .4664664 & 2.38 & 0.017 & 1.113932 & 3.0191 \\
\hline fplt I & 1.703208 & .4140313 & 2.19 & 0.028 & 1.057669 & 2.7427 \\
\hline _cons I & .0033278 & .0068578 & -2.77 & 0.006 & .0000586 & .1889 \\
\hline ln_r 1 & 2.939118 & .4037653 & & & 2.147753 & $3.7304 \varepsilon$ \\
\hline /ln_s I & 1.078573 & .248511 & & & .5915002 & 1.5656 \\
\hline $\begin{array}{ll}r & 1 \\
s & 1\end{array}$ & $\begin{array}{r}18.89918 \\
2.94048\end{array}$ & $\begin{array}{r}7.630832 \\
.7307416\end{array}$ & & & $\begin{array}{l}8.565591 \\
1.806697\end{array}$ & $\begin{array}{l}41.6992 \\
4.7857\end{array}$ \\
\hline
\end{tabular}

LR test vs. pooled: $\operatorname{chibar} 2(01)=161.18$

Prob $>=$ chibar2 $=0.000$

- estimates store BFrandom_sS

- xtnbreg sideswipe rlcinstalled2 lnaadt channelization median leftturn thrulanes plt fplt pplt speed1 speed2, irr fe

Conditional FE negative binomial regression

Group variable: id

$\begin{array}{rrr}\text { Number of obs } & = \\ \text { Number of groups } & = & 685 \\ \text { Obs per group: } & 48 \\ \min & = & 14 \\ \text { avg } & = & 14.3 \\ \max & = & 15 \\ & & \\ \text { Wald chi2(11) } & = & 30.41 \\ \text { Prob > chi2 } & = & 0.0014\end{array}$

Log likelihood $=-828.54026$

Prob > chi2 = 0.0014

\begin{tabular}{|c|c|c|c|c|c|c|c|}
\hline sideswipe & 1 & IRR & Std. Err. & $\mathbf{z}$ & $\mathrm{P}>|\mathbf{z}|$ & [95\% Conf. & Interval] \\
\hline & & & & & & & \\
\hline rlcinstalled2 & 1 & 1. 389411 & .1227235 & 3.72 & 0.000 & 1.168547 & 1.65202 \\
\hline Inaadt & i & 1. 388501 & .3242052 & 1.41 & 0.160 & .8786114 & 2.194299 \\
\hline channelization & i & 14.30891 & 25.45519 & 1.50 & 0.135 & .4378648 & 467.5984 \\
\hline median & i & .198335 & .2153202 & -1.49 & 0.136 & .0236212 & 1.665317 \\
\hline leftturn & 1 & .1510241 & .2264494 & -1.26 & 0.207 & .0079934 & 2.853379 \\
\hline thrulanes & i & .1240258 & .1764009 & -1.47 & 0.142 & .0076358 & 2.014509 \\
\hline plt & 1 & 12.54594 & 18.53453 & 1.71 & 0.087 & .6934174 & 226.9925 \\
\hline fplt & I & 32.50525 & 62.68131 & 1.81 & 0.071 & .7422395 & 1423.518 \\
\hline pplt & I & 1.942345 & 2.462207 & 0.52 & 0.600 & .1619212 & 23.29963 \\
\hline speed1 & I & .9699335 & .0432112 & -0.69 & 0.493 & .8888333 & 1.058434 \\
\hline speed2 & I & 1. 383394 & .2188109 & 2.05 & 0.040 & 1.014639 & 1.886166 \\
\hline _cons & I & $4.51 e-06$ & .0000244 & -2.28 & 0.023 & $1.13 e-10$ & .1807297 \\
\hline
\end{tabular}

estimates store fixed_sideswipe

. hausman fixed_sideswipe BFrandom_sS

\begin{tabular}{|c|c|c|c|c|c|}
\hline & I & (b) & (B) & $(b-B)$ & $\operatorname{sqrt}\left(\operatorname{diag}\left(v_{-} b-v_{-}{ }_{B}\right)\right)$ \\
\hline & I & fixed_side e & BFrandom_SS & Difference & S.E. \\
\hline & & คคคดคด & -ーーーーー- & & \\
\hline rlcinstall 2 & 1 & .3288799 & .2963726 & .0325073 & .0075532 \\
\hline lnaadt & I & .3282251 & .6073413 & -.2791163 & .1332532 \\
\hline channeliza n & i & 2.660882 & .4074996 & 2.253383 & 1.761284 \\
\hline median & I & -1.617798 & -.6014958 & -1.016302 & 1.054892 \\
\hline leftturn & i & -1.890316 & .3475173 & -2.237833 & 1.49018 \\
\hline thrulanes & i & -2.087266 & .4055786 & -2.492844 & 1.41272 \\
\hline plt & i & 2.529397 & .6064332 & 1.922964 & 1.455271 \\
\hline fplt & I & 3.481402 & .5325136 & 2.948888 & 1. 912961 \\
\hline
\end{tabular}

$\mathrm{b}=$ consistent under $\mathrm{Ho}$ and $\mathrm{Ha}$; obtained from xtnbreg $\mathrm{B}=$ inconsistent under Ha, efficient under Ho; obtained from xtnbreg

Test: Ho: difference in coefficients not systematic

$$
\operatorname{chi2}(8)=(b-B) \cdot\left[\left(V_{-} b-V_{-} B\right)^{\wedge}(-1)\right](b-B)
$$


$=-42.36$

chi2 $<0==>$ model fitted on these data fails to meet the asymptotic assumptions of the Hausman test; see suest for a generalized test 


\section{Appendix G - ZINB StataSE Output}

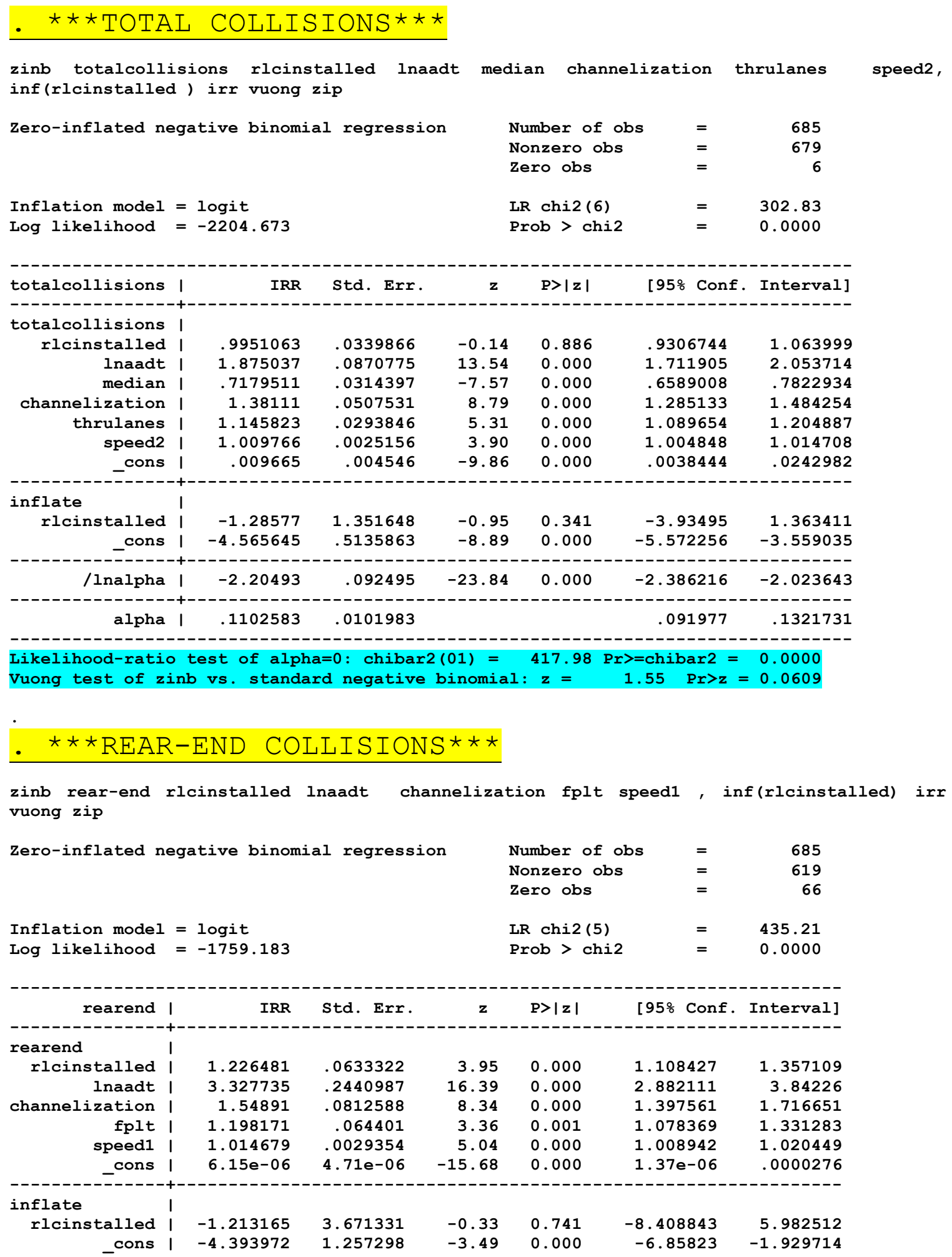




\begin{tabular}{|c|c|c|c|c|c|c|}
\hline /Inalpha I & -1.535985 & .1235649 & -12.43 & 0.000 & -1.778168 & -1.293802 \\
\hline alpha I & .2152436 & .0265966 & & & .1689474 & .2742261 \\
\hline
\end{tabular}

Likelihood-ratio test of alpha=0: chibar2 $(01)=258.11$ Pr>=chibar2 $=0.0000$ Vuong test of $\mathrm{zinb} v \mathrm{vs}$. standard negative binomial: $\mathrm{z}=0.41 \mathrm{Pr}>\mathrm{z}=0.3419$ - $\quad \star \star \star$ ANGLE COLLISIONS $* \star \star$

zinb angle rlcinstalled lnaadt median thrulanes fplt pplt, inf(rlcinstalled) irr vuong zip

$\begin{array}{lllr}\text { Zero-inflated negative binomial regression } & \text { Number of obs } & = & 685 \\ & \begin{array}{l}\text { Nonzero obs } \\ \text { Zero obs }\end{array} & = & 580 \\ & & & 105 \\ \text { Inflation model }=\text { logit } & \text { LR chi2 (6) } & = & 130.41 \\ \text { Log likelihood }=-1475.187 & \text { Prob }>\text { chi2 } & = & 0.0000\end{array}$

\begin{tabular}{|c|c|c|c|c|c|c|}
\hline angle & IRR & Std. Err. & $\mathbf{z}$ & $P>|z|$ & [95\% Conf & Interval] \\
\hline angle & & & & & & \\
\hline rlcinstalled & .776775 & .0536823 & -3.66 & 0.000 & .6783744 & .889449 \\
\hline lnaadt & .9270558 & .0823906 & -0.85 & 0.394 & .7788551 & 1.103456 \\
\hline median & .7671042 & .0618639 & -3.29 & 0.001 & .6549502 & .8984634 \\
\hline thrulanes & 1.275476 & .0617318 & 5.03 & 0.000 & 1.160045 & 1.402393 \\
\hline fplt & .7240195 & .0558351 & -4.19 & 0.000 & .6224538 & .8421576 \\
\hline pplt & .5249256 & .0439406 & -7.70 & 0.000 & .4454974 & .6185151 \\
\hline cons & 6.945149 & 6.299132 & 2.14 & 0.033 & 1.173968 & 41.08724 \\
\hline inflate & & & & & & \\
\hline cinstalled & .0616317 & .6910007 & 0.09 & 0.929 & -1.292705 & 1.415968 \\
\hline _cons & -2.996911 & .483078 & -6.20 & 0.000 & -3.943727 & -2.050096 \\
\hline Ino & & & & & & \\
\hline /lnalpha & -1.342438 & .1685765 & -7.96 & 0.000 & -1.672842 & -1.012034 \\
\hline alpha & .261208 & .0440335 & & & .1877128 & .3634789 \\
\hline
\end{tabular}

Likelihood-ratio test of alpha=0: $\operatorname{chibar2}(01)=119.91 \mathrm{Pr}>=$ chibar2 $=0.0000$ Vuong test of zinb vs. standard negative binomial: $z=1.15$ Pr>z=0.1244

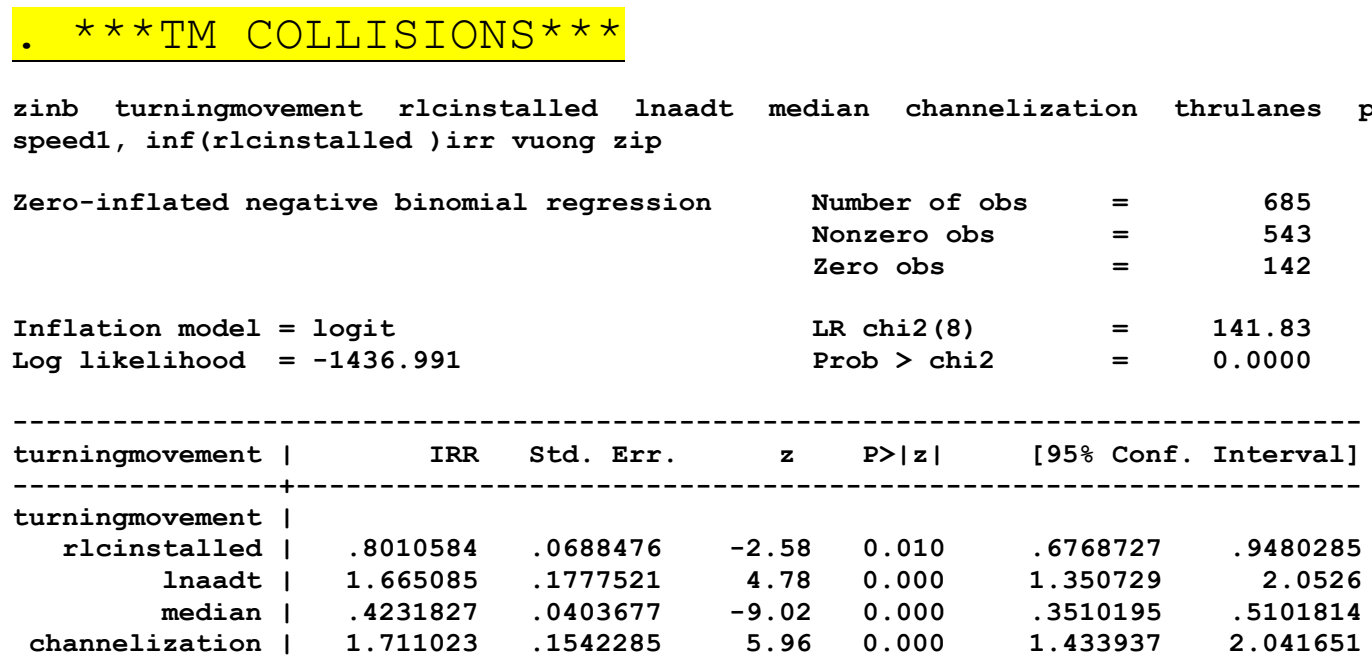




\begin{tabular}{|c|c|c|c|c|c|c|}
\hline thrulanes & 1.159845 & .0662391 & 2.60 & 0.009 & 1.037021 & 1.297216 \\
\hline plt & 1.311346 & .115588 & 3.08 & 0.002 & 1.103287 & 1.558641 \\
\hline pplt & 2.020945 & .1695462 & 8.39 & 0.000 & 1.714523 & 2. 382131 \\
\hline speed1 & .9938945 & .004524 & -1.35 & 0.178 & .985067 & 1.002801 \\
\hline _cons & .0144313 & .0160821 & -3.80 & 0.000 & .0016246 & .1281956 \\
\hline late & & & & & & \\
\hline alled & 18.27494 & 20199.76 & 0.00 & 0.999 & -39572.53 & 39609.08 \\
\hline cons & -22.72116 & 20199.76 & -0.00 & 0.999 & -39613.52 & 39568.08 \\
\hline /lnalpha & -.7174442 & .1234193 & -5.81 & 0.000 & -.9593416 & -.4755468 \\
\hline alpha & .4879979 & .0602284 & & & .3831451 & .6215451 \\
\hline
\end{tabular}

Likelihood-ratio test of alpha=0: chibar2 $(01)=183.13$ Pr>=chibar2 $=0.0000$ Vuong test of $\mathbf{z i n b}$ vs. standard negative binomial: $z=0.13$ Pr>z=0.4475

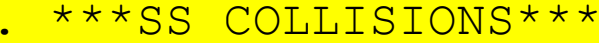

zinb sideswipe rlcinstalled lnaadt median channelization leftturn thrulanes plt fplt, inf (rlcinstalled) irr vuong zip

zero-inflated negative binomial regression

$\begin{array}{llr}\text { Number of obs } & = & 685 \\ \text { Nonzero obs } & = & 435 \\ \text { Zero obs } & = & 250 \\ & & \\ \text { LR chi2 (8) } & = & 144.52 \\ \text { Prob > chi2 } & = & 0.0000\end{array}$

Inflation model $=$ logit

Log likelihood $=-1098.651$

Prob > chi2

[95\% Conf. Interval]

\begin{tabular}{|c|c|c|c|c|c|c|c|}
\hline sideswipe & I & IRR & Std. Err. & $\mathbf{z}$ & $P>|z|$ & [95\% Conf. & Interval] \\
\hline ideswipe & 1 & & & & & & \\
\hline rlcinstalled & i & 1.175147 & .1011541 & 1.87 & 0.061 & .9927101 & 1.391111 \\
\hline Inaadt & i & 2.567085 & .3230318 & 7.49 & 0.000 & 2.005988 & 3.285126 \\
\hline median & 1 & .5659645 & .065674 & -4.91 & 0.000 & .4508339 & .7104963 \\
\hline channelization & 1 & 1.485314 & .1632713 & 3.60 & 0.000 & 1.197433 & 1.842407 \\
\hline leftturn & 1 & 1.481704 & .1064526 & 5.47 & 0.000 & 1.287085 & 1.705751 \\
\hline thrulanes & i & 1.520448 & .106941 & 5.96 & 0.000 & 1.324653 & 1.745184 \\
\hline plt & i & 1.816013 & .2140129 & 5.06 & 0.000 & 1.441474 & 2.287869 \\
\hline fplt & 1 & 1.43505 & .1579344 & 3.28 & 0.001 & 1.156613 & 1.780517 \\
\hline _cons & I & .0000165 & .0000216 & -8.45 & 0.000 & $1.29 e-06$ & .0002129 \\
\hline nflate & I & & & & & & \\
\hline rlcinstalled & i & -12.23251 & 115798.9 & -0.00 & 1.000 & -226973.8 & 226949.4 \\
\hline _cons & 1 & -12.78321 & 427.8575 & -0.03 & 0.976 & -851.3685 & 825.802 \\
\hline /Inalpha & 1 & -.7177857 & .1400877 & -5.12 & 0.000 & -.9923525 & -.4432189 \\
\hline & & & & & & & \\
\hline alpha & 1 & .4878313 & .0683391 & & & .3707036 & .6419666 \\
\hline
\end{tabular}

Likelihood-ratio test of alpha=0: chibar2 $(01)=67.01$ Pr $>=$ chibar2 $=0.0000$

Vuong test of $z$ inb vs. standard negative binomial: $z=-0.01$ Pr>z=0.5024 


\section{Appendix H - Models with Interaction Terms}

\section{TOTAL COLLISIONS}

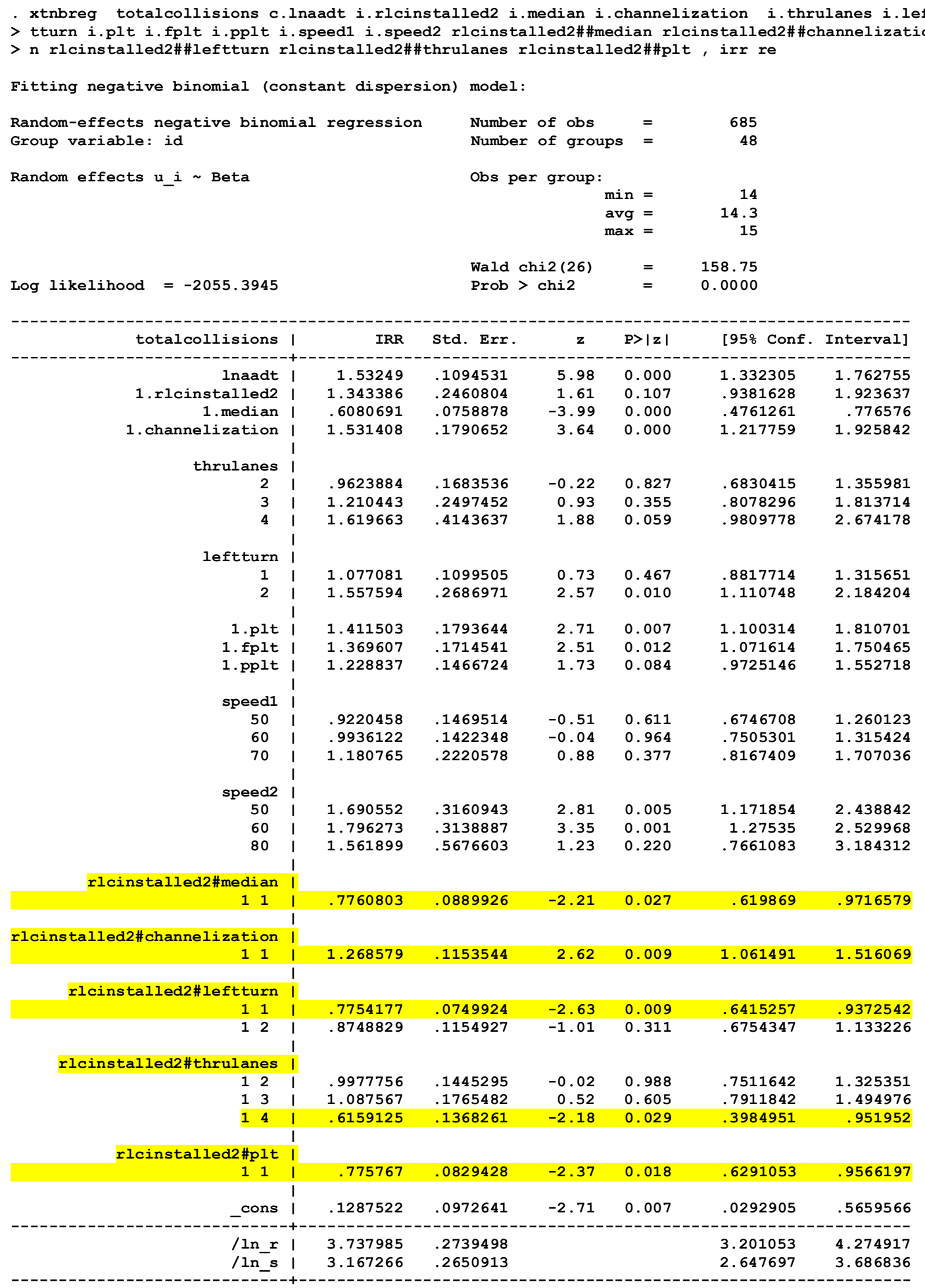




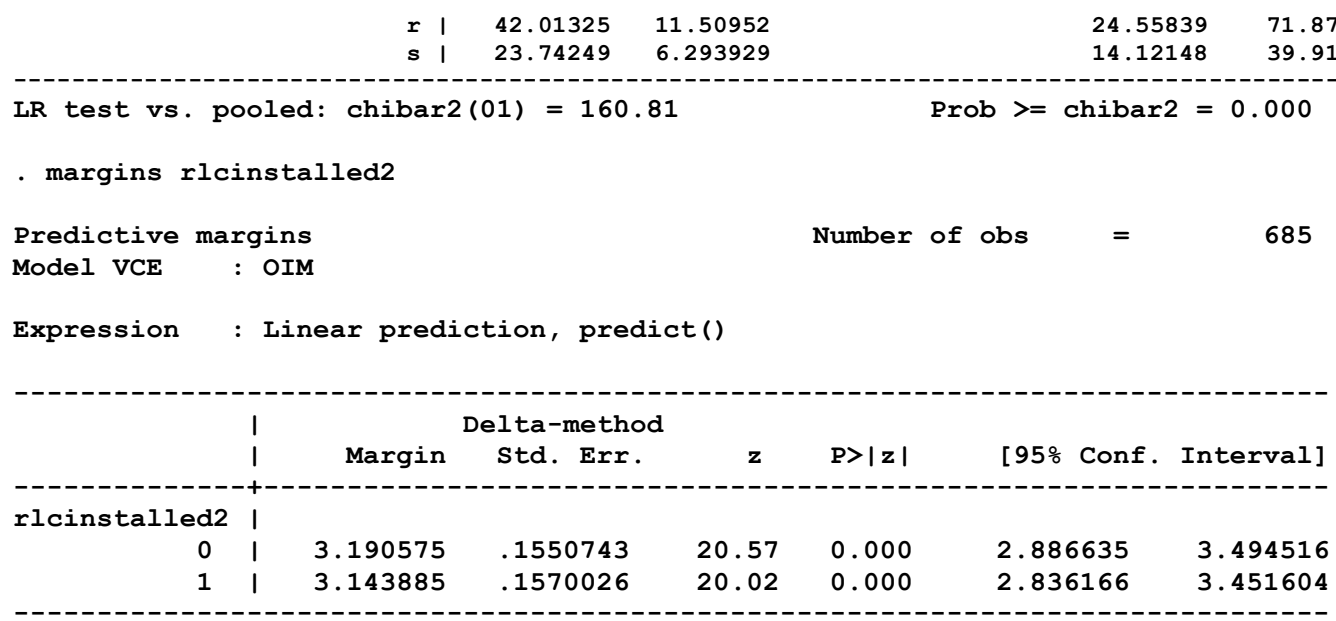

* *margins r.rlcinstalled2 ... same results as below:

- margins, dydx(rlcinstalled2)

Average marginal effects $\quad$ Number of obs $=685$ Model VCE : OIM

Expression : Linear prediction, predict()

$\mathrm{dy} / \mathrm{dx}$ w.r.t. : 1.rlcinstalled2

\begin{tabular}{|c|c|c|c|c|c|c|c|}
\hline \multirow{2}{*}{\multicolumn{2}{|c|}{ 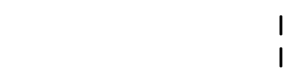 }} & \multicolumn{6}{|c|}{ Delta-method } \\
\hline & & $d y / d x$ & std. Err. & $\mathbf{z}$ & $\mathrm{P}>|\mathbf{z}|$ & [95\% Conf. & Interval] \\
\hline---------------1 & -+ & ------ & ----------1 & ------ & -ー-ー-ー & ----------1 & --------- \\
\hline 1.rlcinstalled2 & 1 & 466905 & .0420488 & -1.11 & 0.267 & -.1291046 & .0357236 \\
\hline
\end{tabular}

Note: $d y / d x$ for factor levels is the discrete change from the base level.

- margins rlcinstalled2\#\#median rlcinstalled2\#\#channelization rlcinstalled2\#\#leftturn rlcinstalled2\#\#thrulanes rlcinstalled2\#\#plt

Predictive margins $\quad$ Number of obs $=685$

Model VCE : OIM

Expression : Linear prediction, predict()

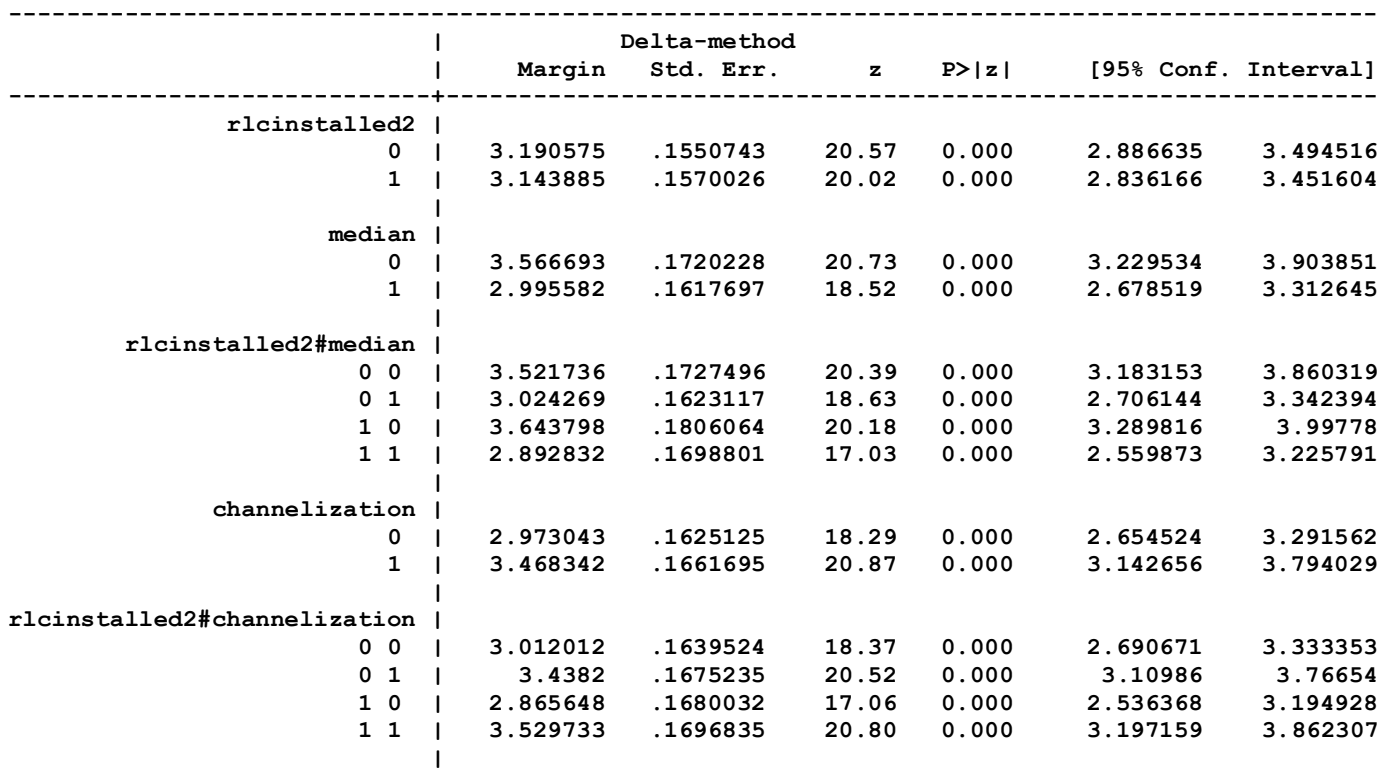




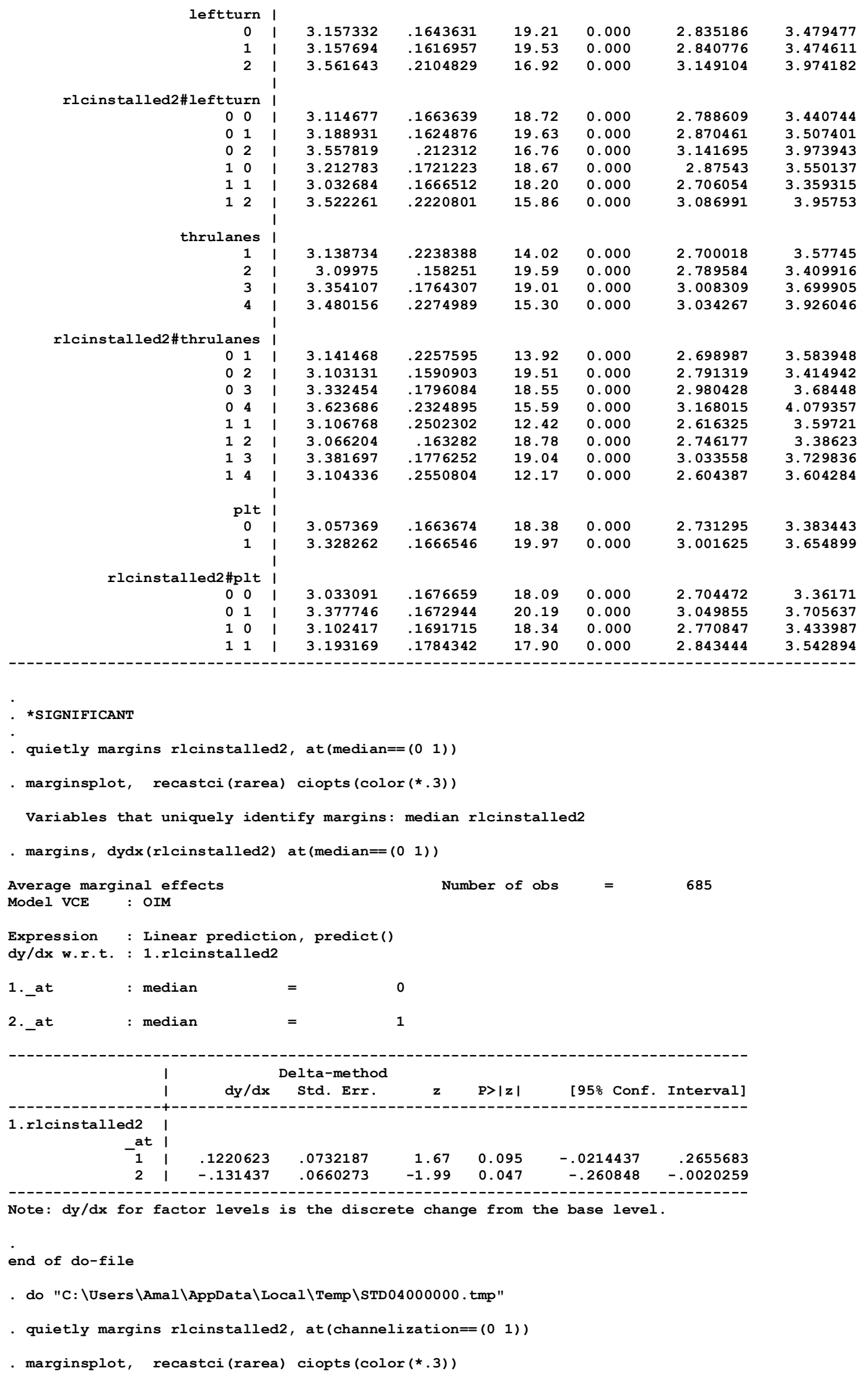




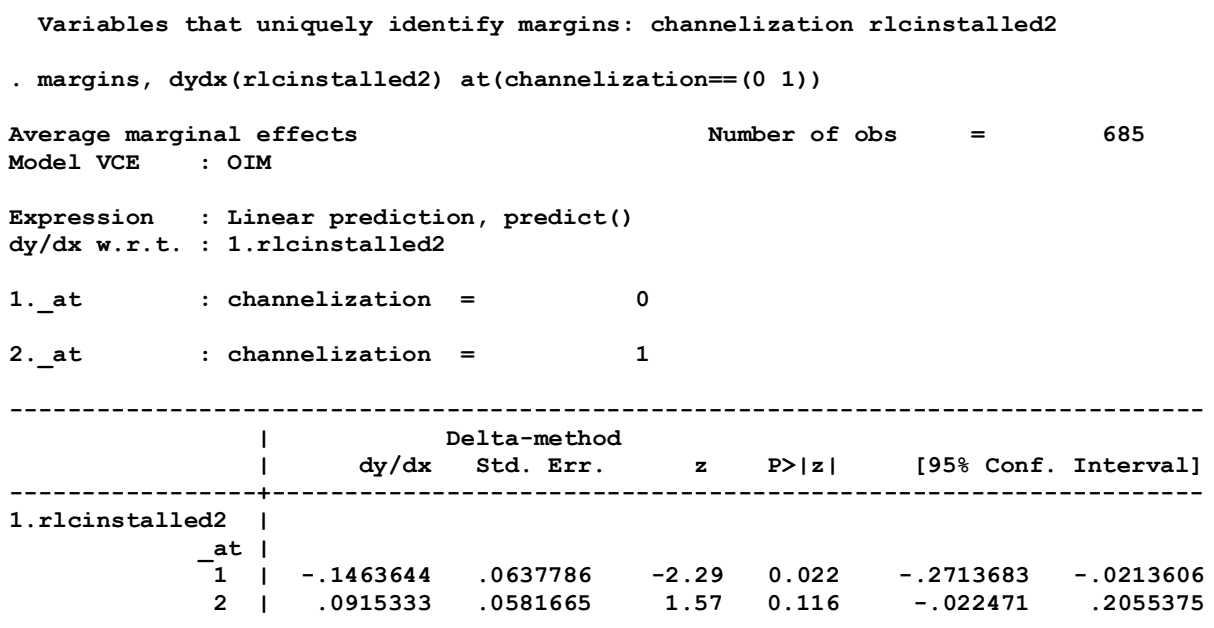

Note: $d y / d x$ for factor levels is the discrete change from the base level.

end of do-file

. do "C: \Users\Amal\AppData\Local\Temp\STD04000000.tmp"

. quietly margins rlcinstalled2, at(leftturn==(0 (1) 2))

- marginsplot, recastci (rarea) ciopts (color(*.3))

Variables that uniquely identify margins: leftturn rlcinstalled2

margins, dydx(rlcinstalled2) at(leftturn==( $\left.\begin{array}{ll}0 & (1)\end{array}\right)$ )

Average marginal effects $\quad$ Number of obs $=685$

Model VCE : OIM

Expression : Linear prediction, predict()

dy/dx w.r.t. : 1.rlcinstalled2

1._at : lefturn $\quad=\quad 0$

2._at : lefturn $\quad=\quad 1$

3. at : lefturn $\quad 2$

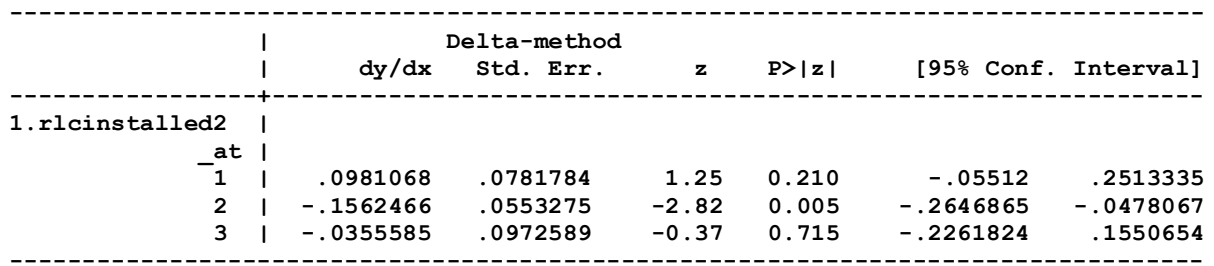

Note: $d y / d x$ for factor levels is the discrete change from the base level.

end of do-file

. do "C:\Users\Amal\AppData\Local\Temp\STD04000000.tmp"

- quietly margins rlcinstalled2, at(thrulanes==(2 (1) 4))

marginsplot, recastci (rarea) ciopts (color(*.3))

Variables that uniquely identify margins: thrulanes rlcinstalled2

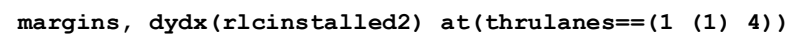

Average marginal effects

Number of obs =

685

Model VCE : OIM

Expression : Linear prediction, predict()

dy/dx w.r.t. : 1.rlcinstalled2 


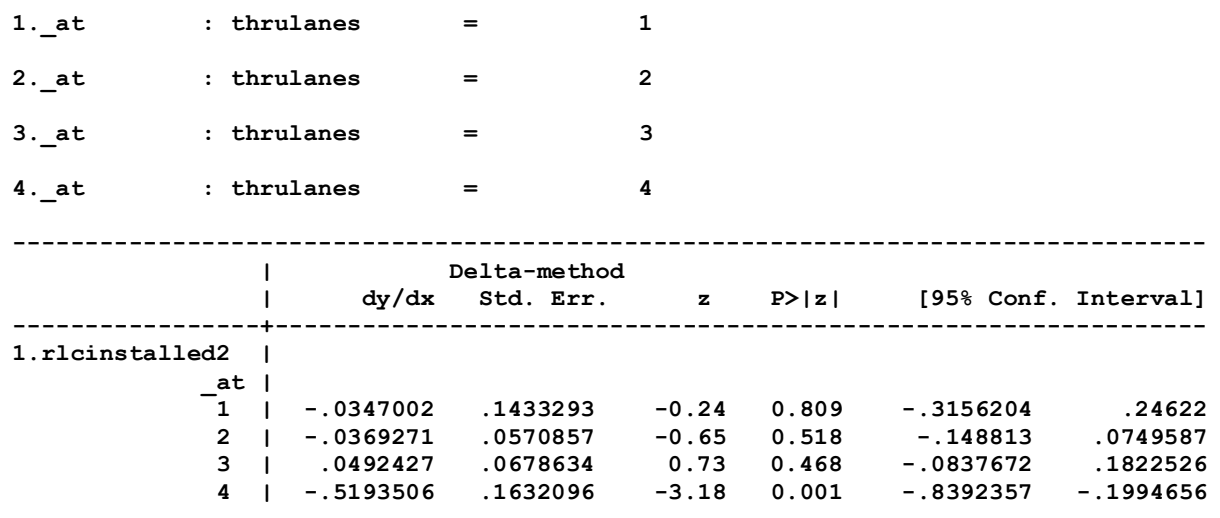

Note: $d y / d x$ for factor levels is the discrete change from the base level.

end of do-file

. do "C: \Users\Amal\AppData\Local\Temp\STD04000000.tmp"

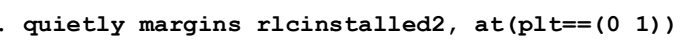

marginsplot, recastci(rarea) ciopts (color(*.3))

Variables that uniquely identify margins: plt rlcinstalled2

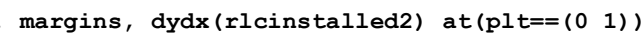

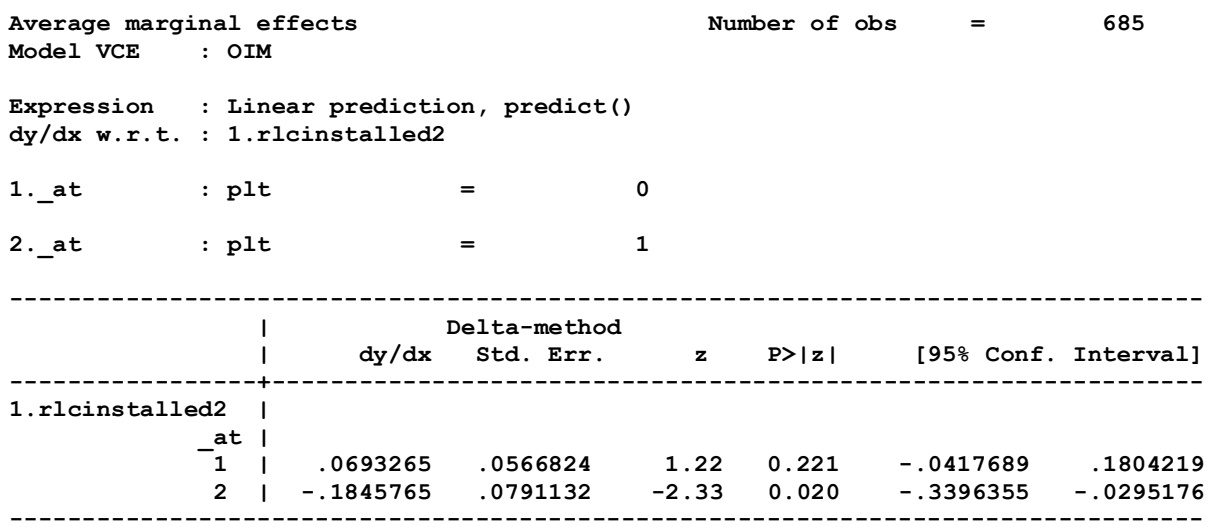

Note: $d y / d x$ for factor levels is the discrete change from the base level.

end of do-file

. do "C: \Users\Amal\AppData\Local\Temp\STD04000000.tmp"

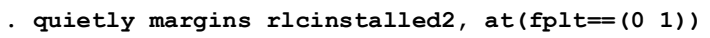

- marginsplot, recastci(rarea) ciopts(color(*.3))

Variables that uniquely identify margins: fplt rlcinstalled2

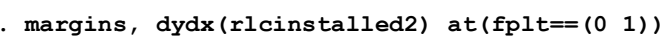

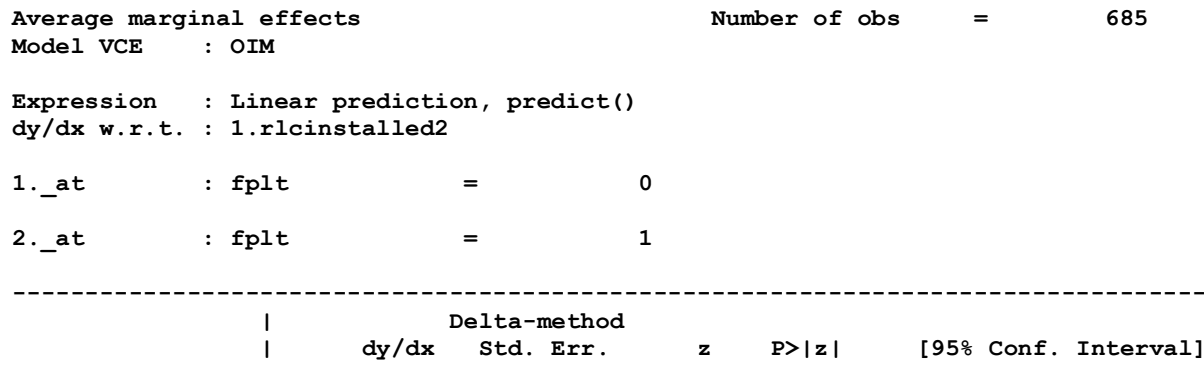




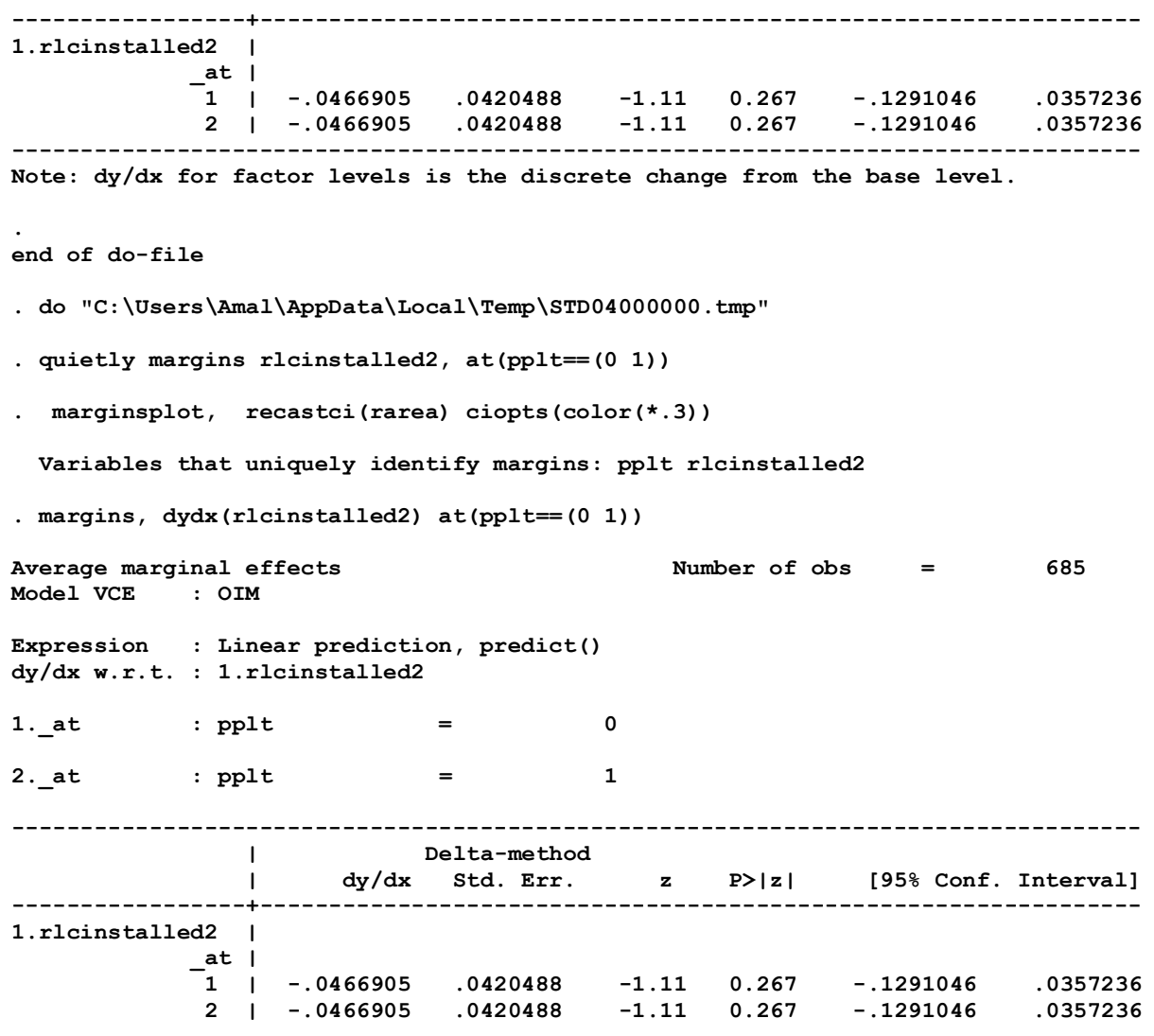

Note: dy/dx for factor levels is the discrete change from the base level.

end of do-file

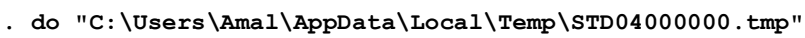

- quietly margins rlcinstalled2, at (speed2 $\left.=\left(\begin{array}{lll}40 & (10) & 80\end{array}\right)\right)$

at values for factor speed2 do not sum to 1

$r(198)$;

end of do-file

$r(198)$;

. do "C: \Users \Amal\AppData\Local\Temp\STD04000000.tmp"

. quietly margins rlcinstalled2, at (speed1 $==\left(\begin{array}{lll}40 & \text { (10) 70) })\end{array}\right.$

- marginsplot, recastci(rarea) ciopts(color(*.3))

Variables that uniquely identify margins: speed1 rlcinstalled2

margins, dydx(rlcinstalled2) at (speed1 $==(40$ (10) 70) $)$

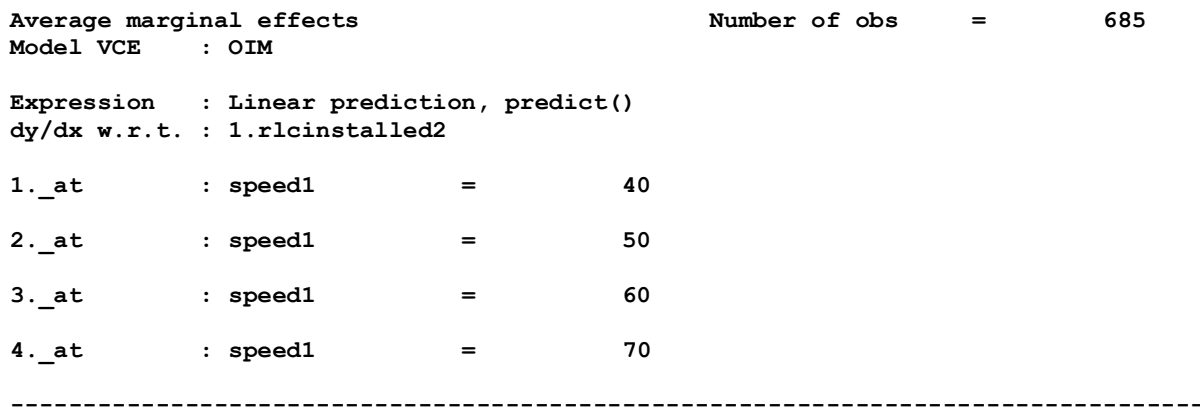

I Delta-method 


\begin{tabular}{|c|c|c|c|c|c|c|c|}
\hline & 1 & $\mathrm{dy} / \mathrm{d} \mathbf{x}$ & Std. Err. & $\mathbf{z}$ & $P>|z|$ & [95\% Conf. & Interval] \\
\hline 1 led2 & 1 & & & & & & \\
\hline at & I & & & & & & \\
\hline 1 & i & -.0466905 & .0420488 & -1.11 & 0.267 & -.1291046 & .0357236 \\
\hline 2 & i & -.0466905 & .0420488 & -1.11 & 0.267 & -.1291046 & .0357236 \\
\hline 3 & i & -.0466905 & .0420488 & -1.11 & 0.267 & -.1291046 & .0357236 \\
\hline 4 & i & -.0466905 & .0420488 & -1.11 & 0.267 & -.1291046 & .0357236 \\
\hline
\end{tabular}

Note: $\mathrm{dy} / \mathrm{dx}$ for factor levels is the discrete change from the base level.

end of do-file

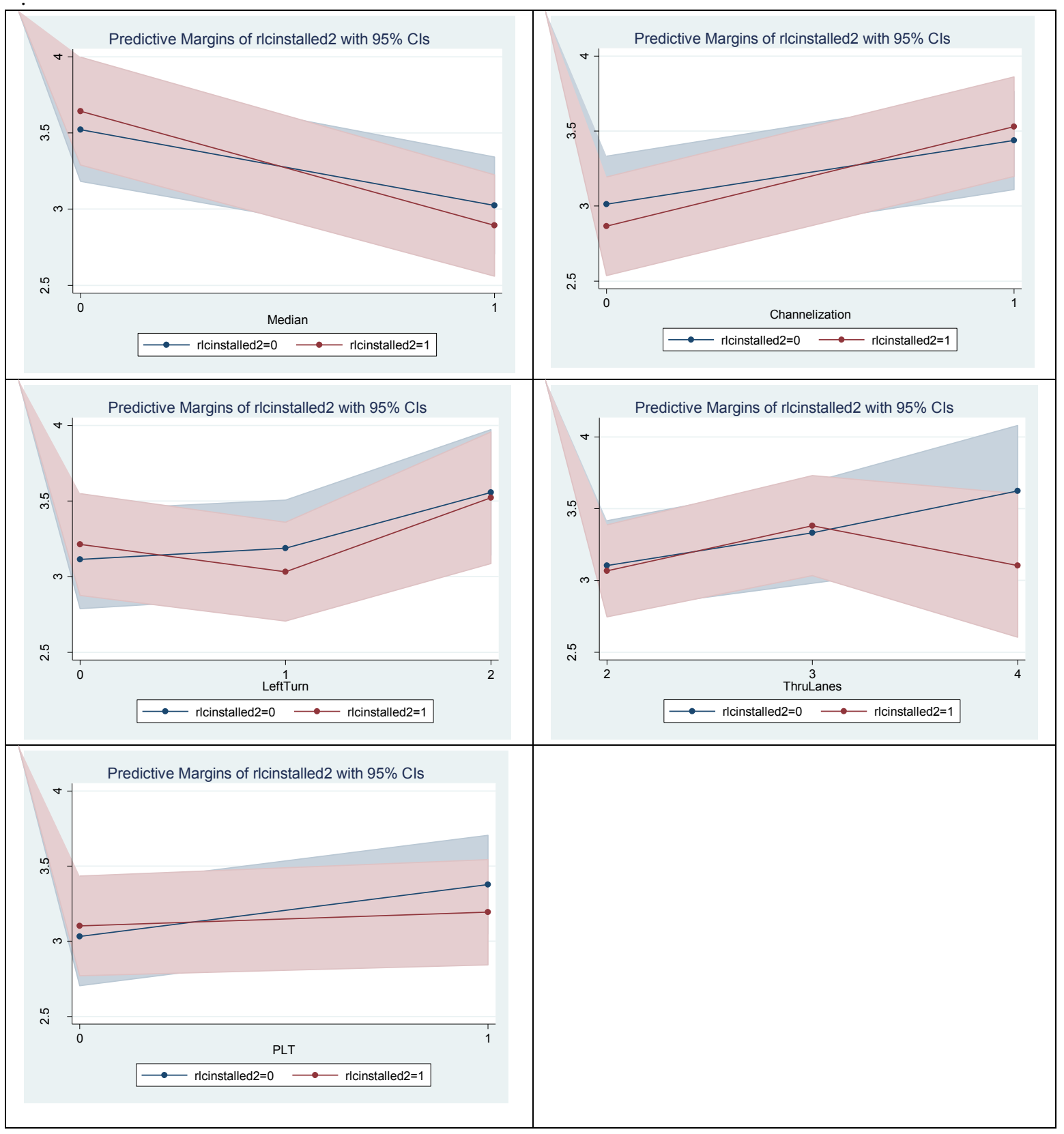




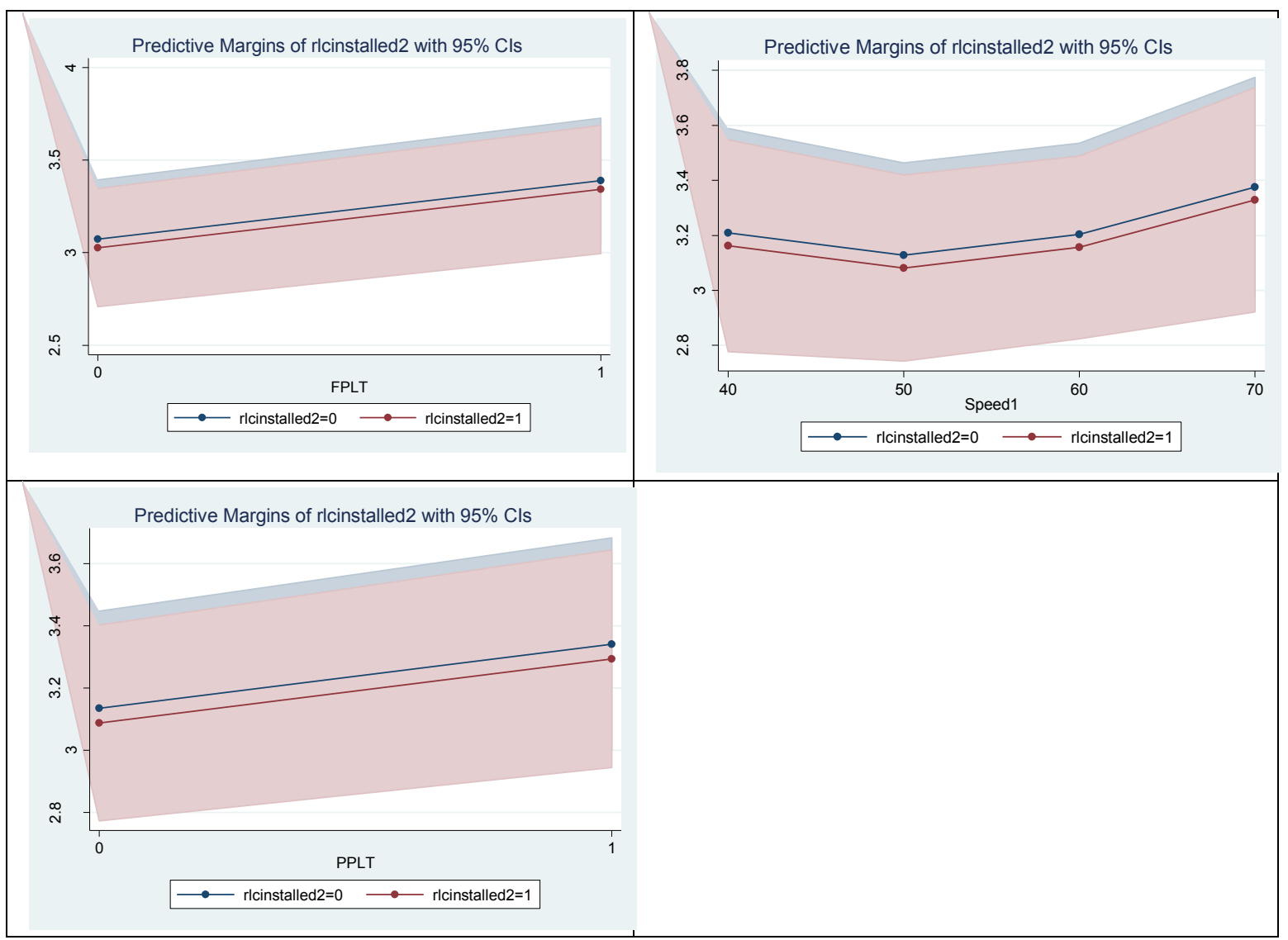

. do "C: \Users\Amal\AppData\Local\Temp\STD04000000.tmp"

- xtnbreg rearend c.lnaadt i.rlcinstalled2 i.median i.channelization i.thrulanes i.leftturn i. > plt i.fplt i.pplt i.speed1 i.speed2 rlcinstalled2\#\#channelization, irr

Fitting negative binomial (constant dispersion) model:

Random-effects negative binomial regression Group variable: id

$\begin{array}{rrr}\text { Number of obs } & = & 685 \\ \text { Number of groups } & = & 48 \\ \text { Obs per group: } \min & = & 14 \\ \text { avg } & = & 14.3 \\ \max & = & 15 \\ & & \\ \text { Wald chi2(19) } & = & 149.03 \\ \text { Prob }>\text { chi2 } & = & 0.0000\end{array}$

$\begin{array}{llll}\text { Log likelihood }=-1598.9703 & \text { Wald chi2(19) } & = & 149.03 \\ \text { Prob }>\text { chi2 } & = & 0.0000\end{array}$

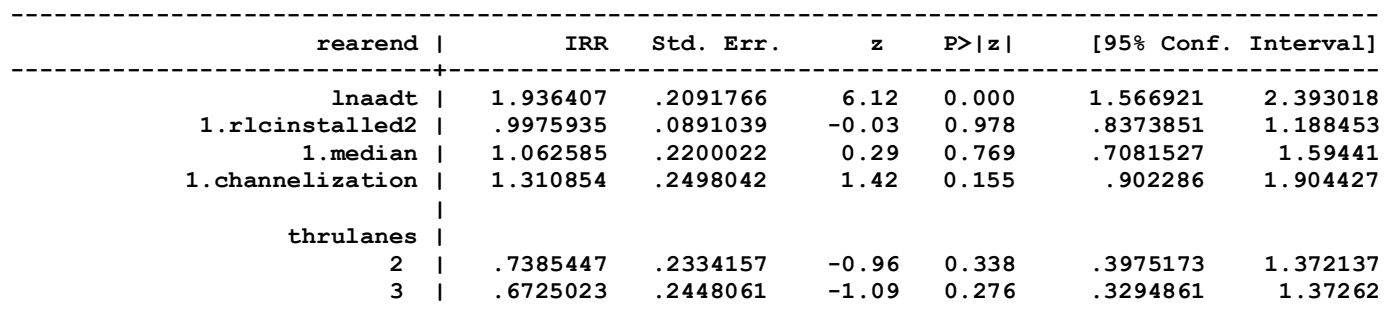




\begin{tabular}{|c|c|c|c|c|c|c|c|}
\hline 4 & 1 & .7068213 & .3094912 & -0.79 & 0.428 & .2996398 & 1.667323 \\
\hline & \multicolumn{5}{|c|}{ leftturn | } & & \\
\hline 1 & 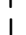 & 1.048321 & .1719149 & 0.29 & 0.774 & .7601599 & 1.445717 \\
\hline \multirow[t]{2}{*}{2} & I & 1.287175 & .361411 & 0.90 & 0.369 & .7424012 & 2.231704 \\
\hline & I & & & & & & \\
\hline 1.plt & I & 1.298995 & .2657121 & 1.28 & 0.201 & .8699467 & 1.939647 \\
\hline 1. fplt & i & 1.716497 & .3610475 & 2.57 & 0.010 & 1.136584 & 2.592296 \\
\hline \multirow[t]{2}{*}{ 1.pplt } & I & 1.331785 & .2694524 & 1.42 & 0.157 & .8958094 & 1.979944 \\
\hline & I & & & & & & \\
\hline \multicolumn{8}{|c|}{ speed1 i } \\
\hline 50 & i & .8366068 & .2207421 & -0.68 & 0.499 & 4988022 & 1.403183 \\
\hline 60 & i & .8595634 & .2098294 & -0.62 & 0.535 & .5327087 & 1.386967 \\
\hline \multirow{2}{*}{70} & i & 2.00273 & .638016 & 2.18 & 0.029 & 1.072636 & 3.739318 \\
\hline & i & & & & & & \\
\hline \multicolumn{8}{|c|}{ speed2 | } \\
\hline 50 & i & .9672 & .3068262 & -0.11 & 0.916 & .5193838 & 1.801126 \\
\hline 60 & i & 1.665909 & .4858255 & 1.75 & 0.080 & .9406263 & 2.95043 \\
\hline \multirow{2}{*}{$\begin{array}{r}80 \\
\end{array}$} & I & 1.920101 & 1.183184 & 1.06 & 0.290 & .5738595 & 6.424546 \\
\hline & 1 & & & & & & \\
\hline \multicolumn{8}{|l|}{ rlcinstalled2\#channelization | } \\
\hline \multirow[t]{2}{*}{11} & I & 1.392687 & .1481322 & 3.11 & 0.002 & 1.130619 & 1.715501 \\
\hline & 1 & & & -3.65 & 0.000 & 0011141 & 1284283 \\
\hline cons & 1 & .0119615 & .0144864 & -3.65 & 0.000 & $.0001+41$ & $.0208=00$ \\
\hline \multirow{2}{*}{\multicolumn{2}{|c|}{$\begin{array}{ll}\ln n_{-} x & 1 \\
\ln \ln _{s} & 1\end{array}$}} & 3.285772 & .3281859 & & & 2.642539 & 3.929004 \\
\hline & & 1.877734 & .2536389 & & & 1.380611 & 2.374857 \\
\hline \multirow{2}{*}{$r$} & I & 26.72961 & 8.77228 & & & 14.04883 & 50.85632 \\
\hline & 1 & 6.53867 & 1.658461 & & & 3.97733 & 10.74948 \\
\hline
\end{tabular}

LR test vs. pooled: chibar2 $(01)=202.61$

Prob $>=$ chibar2 $=0.000$

. margins rlcinstalled2

$\begin{array}{lll}\text { Predictive margins } & \text { Number of obs } \quad=685\end{array}$

Model VCE : OIM

Expression : Linear prediction, predict()

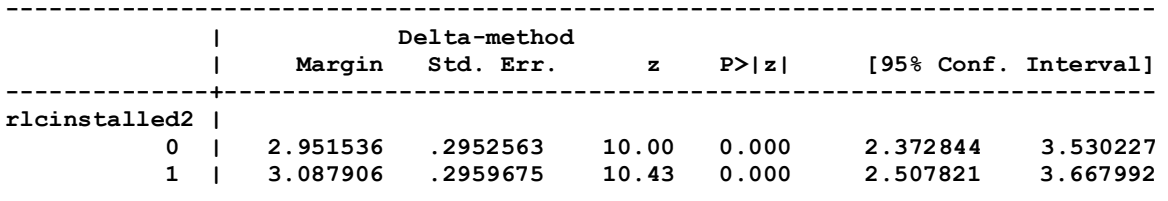

*margins r.rlcinstalled2 ... same results as below:

margins, dydx(rlcinstalled2)

Average marginal effects $\quad$ Number of obs $\quad=\quad 685$

Model VCE : OIM

Expression : Linear prediction, predict()

$\mathrm{dy} / \mathrm{dx}$ w.r.t. : 1.rlcinstalled2

\begin{tabular}{|c|c|c|c|c|c|c|c|}
\hline & 1 & & Delta-method & & & & \\
\hline & 1 & $d y / d x$ & Std. Err. & $\mathbf{z}$ & $P>|z|$ & [95\% Conf. & Interval] \\
\hline 1.rlcinstalled2 & I & .1363708 & .0572834 & 2.38 & 0.017 & .0240973 & .2486442 \\
\hline
\end{tabular}

Note: $d y / d x$ for factor levels is the discrete change from the base level.

- margins rlcinstalled2\#channelization

Predictive margins

Number of obs $\quad=\quad 685$

Model VCE : OIM

Expression : Linear prediction, predict()

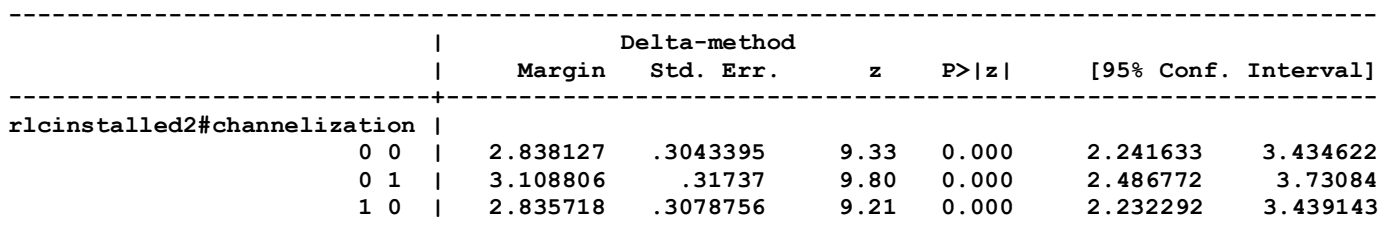




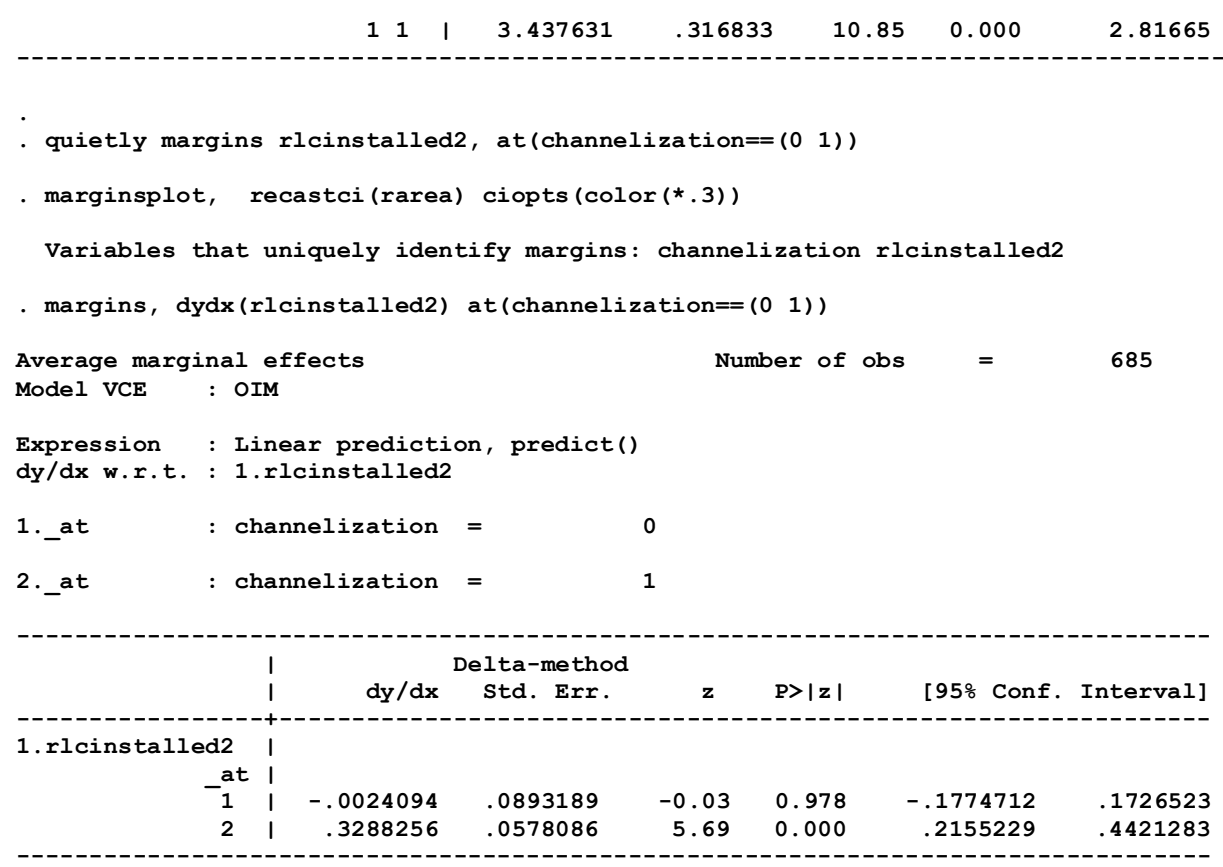

Note: $d y / d x$ for factor levels is the discrete change from the base level.

end of do-file

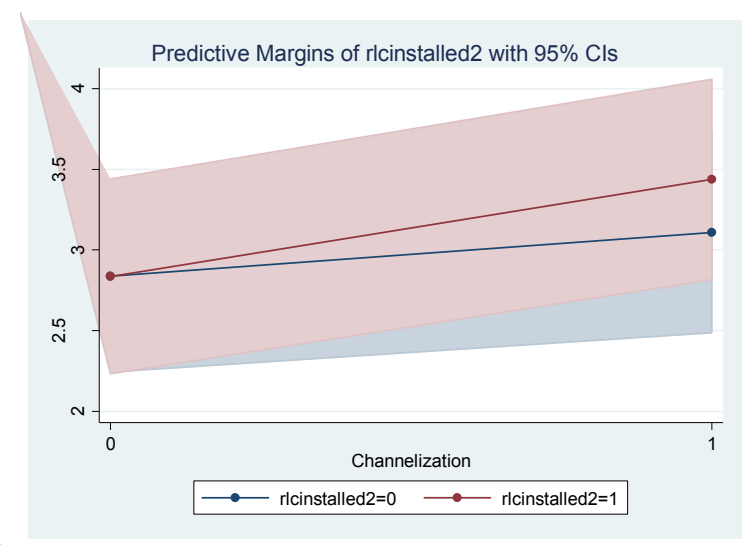

- xtnbreg angle c.lnaadt i.rlcinstalled2 i.median i.channelization i.thrulanes i.leftturn i.pl $>t$ i.fplt $i$.pplt i.speed1 i.speed2 rlcinstalled2\#\#median rlcinstalled2\#\#plt rlcinstalled2\#\#pplt $>$, irr

Fitting negative binomial (constant dispersion) model:

Random-effects negative binomial regression Group variable: id

Random effects $u_{-} i \sim$ Beta

Log likelihood $=-1371.8761$

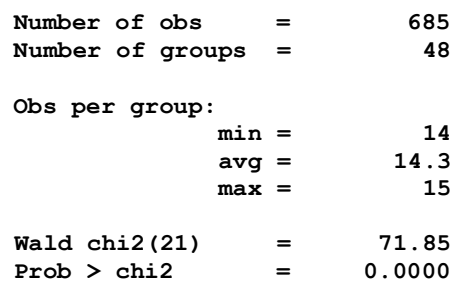

\begin{tabular}{|c|c|c|c|c|c|c|}
\hline angle I & IRR & Std. Err. & z & $P>|z|$ & [95\% Conf. & Interval] \\
\hline rlcin & $\begin{array}{l}1.076626 \\
1.598521\end{array}$ & 154 & $\begin{array}{l}0.51 \\
1.85\end{array}$ & 0.608 & 811956 & 1 \\
\hline $\begin{array}{r}\text { rcinstalled2 } \\
1 . \text { median }\end{array}$ & $\begin{array}{l}1.598521 \\
.6440655\end{array}$ & $\begin{array}{l}.4063099 \\
.1439257\end{array}$ & $\begin{array}{r}1.85 \\
-1.97\end{array}$ & $\begin{array}{l}0.065 \\
0.049\end{array}$ & $\begin{array}{l}.9713175 \\
.4156394\end{array}$ & $\begin{array}{l}2.630726 \\
.9980293\end{array}$ \\
\hline
\end{tabular}




\begin{tabular}{|c|c|c|c|c|c|c|c|}
\hline 1. channelization & 1 & 1.634155 & .3345162 & 2.40 & 0.016 & 1.094082 & 2.440825 \\
\hline \multicolumn{8}{|c|}{ thrulanes I } \\
\hline 2 & i & 1.927561 & .6024654 & 2.10 & 0.036 & 1.04463 & 3.556755 \\
\hline 3 & 1 & 3.215375 & 1.165582 & 3.22 & 0.001 & 1.580042 & 6.543264 \\
\hline \multirow[t]{2}{*}{4} & $\mathrm{i}$ & 2.564371 & 1.220808 & 1.98 & 0.048 & 1.00868 & 6.519414 \\
\hline & i & & & & & & \\
\hline \multicolumn{8}{|c|}{ leftturn } \\
\hline 1 & 1 & 1.219535 & .2215532 & 1.09 & 0.275 & .8541932 & 1.741134 \\
\hline \multirow{2}{*}{2} & 1 & 1.865167 & .5717297 & 2.03 & 0.042 & 1.022819 & 3.401235 \\
\hline & i & & & & & & \\
\hline 1.plt & $\mathrm{i}$ & 1.353603 & .3001375 & 1.37 & 0.172 & .876501 & 2.090404 \\
\hline 1. fplt & $\mathrm{i}$ & .7266561 & .1632433 & -1.42 & 0.155 & .4678498 & 1.128629 \\
\hline \multirow{2}{*}{1. pplt } & i & .6334003 & .13648 & -2.12 & 0.034 & .4152103 & .9662474 \\
\hline & $\mathrm{i}$ & & & & & & \\
\hline \multicolumn{8}{|c|}{ speed1 } \\
\hline 50 & 1 & 1.09475 & .3205495 & 0.31 & 0.757 & .6167054 & 1.943354 \\
\hline \multirow{3}{*}{$\begin{array}{l}60 \\
70\end{array}$} & i & 1.46962 & .3956364 & 1.43 & 0.153 & .8670679 & 2.490904 \\
\hline & 1 & .7084149 & .2555858 & -0.96 & 0.339 & .3492903 & 1.436775 \\
\hline \multirow{2}{*}{\multicolumn{8}{|c|}{ speed2 }} \\
\hline & 1 & & & & & & \\
\hline 50 & 1 & 1.469772 & .5182663 & 1.09 & 0.275 & .7363804 & 2.93358 \\
\hline \multirow{3}{*}{80} & 1 & 1.209949 & .403874 & 0.57 & 0.568 & .6289858 & 2. 327519 \\
\hline & $\mathrm{i}$ & .7297715 & .4995172 & -0.46 & 0.645 & .1907888 & 2.791393 \\
\hline & 1 & & & & & & \\
\hline \multicolumn{8}{|l|}{ rlcinstalled2\#median } \\
\hline \multirow[t]{2}{*}{11} & i & .4845125 & .1127393 & -3.11 & 0.002 & .3070734 & .7644828 \\
\hline & i & & & & & & \\
\hline \multicolumn{7}{|l|}{ rlcinstalled2\#plt i } & \\
\hline \multirow[t]{2}{*}{11} & 1 & .4841608 & .1185386 & -2.96 & 0.003 & .2996314 & .7823334 \\
\hline & 1 & & & & & & \\
\hline \multicolumn{8}{|l|}{ rlcinstalled2\#pplt i } \\
\hline 11 & 1 & 4991649 & .1308708 & -2.65 & 0.008 & .2985908 & .8344717 \\
\hline _cons & $\begin{array}{l}1 \\
1\end{array}$ & 1.415376 & 2.137256 & 0.23 & 0.818 & .0733726 & 27.30295 \\
\hline \multirow{2}{*}{\multicolumn{2}{|c|}{$\begin{array}{l}/ \ln \_r \\
/ n_{n} s\end{array}$}} & 2.848562 & .3008054 & & & 2.258994 & 3.43813 \\
\hline & & 1.775826 & .2617494 & & & 1.262807 & 2.288845 \\
\hline$r$ & 1 & 17.26294 & 5.192784 & & & 9.573455 & 31.12868 \\
\hline $\mathbf{s}$ & 1 & 5.905157 & 1.545671 & & & 3.53533 & 9.863543 \\
\hline
\end{tabular}

LR test vs. pooled: chibar2(01) $=144.34$

Prob $>=$ chibar2 $=0.000$

. margins rlcinstalled2

Predictive margins

Number of obs=

685

Model VCE : OIM

Expression : Linear prediction, predict()

\begin{tabular}{|c|c|c|c|c|c|c|c|}
\hline & 1 & & Delta-method & & & & \\
\hline & 1 & Margin & Std. Err. & $\mathbf{z}$ & $P>|z|$ & [95\% Conf. & Interval] \\
\hline \multicolumn{8}{|c|}{ rlcinstalled2 | } \\
\hline 0 & I & 2.195495 & .2076531 & 10.57 & 0.000 & 1.788503 & 2.602488 \\
\hline 1 & 1 & 1.662107 & .2176976 & 7.63 & 0.000 & 1.235427 & 2.088786 \\
\hline
\end{tabular}

*margins $r . r l c i n s t a l l e d 2 \ldots$ same results as below:

margins, dydx(rlcinstalled2)

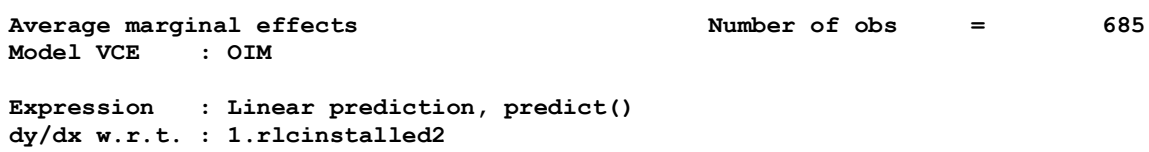

|
| dy/dx Std. Err.

Note: $d y / d x$ for factor levels is the discrete change from the base level.

- margins rlcinstalled2\#\#median rlcinstalled2\#\#plt rlcinstalled2\#\#pplt 


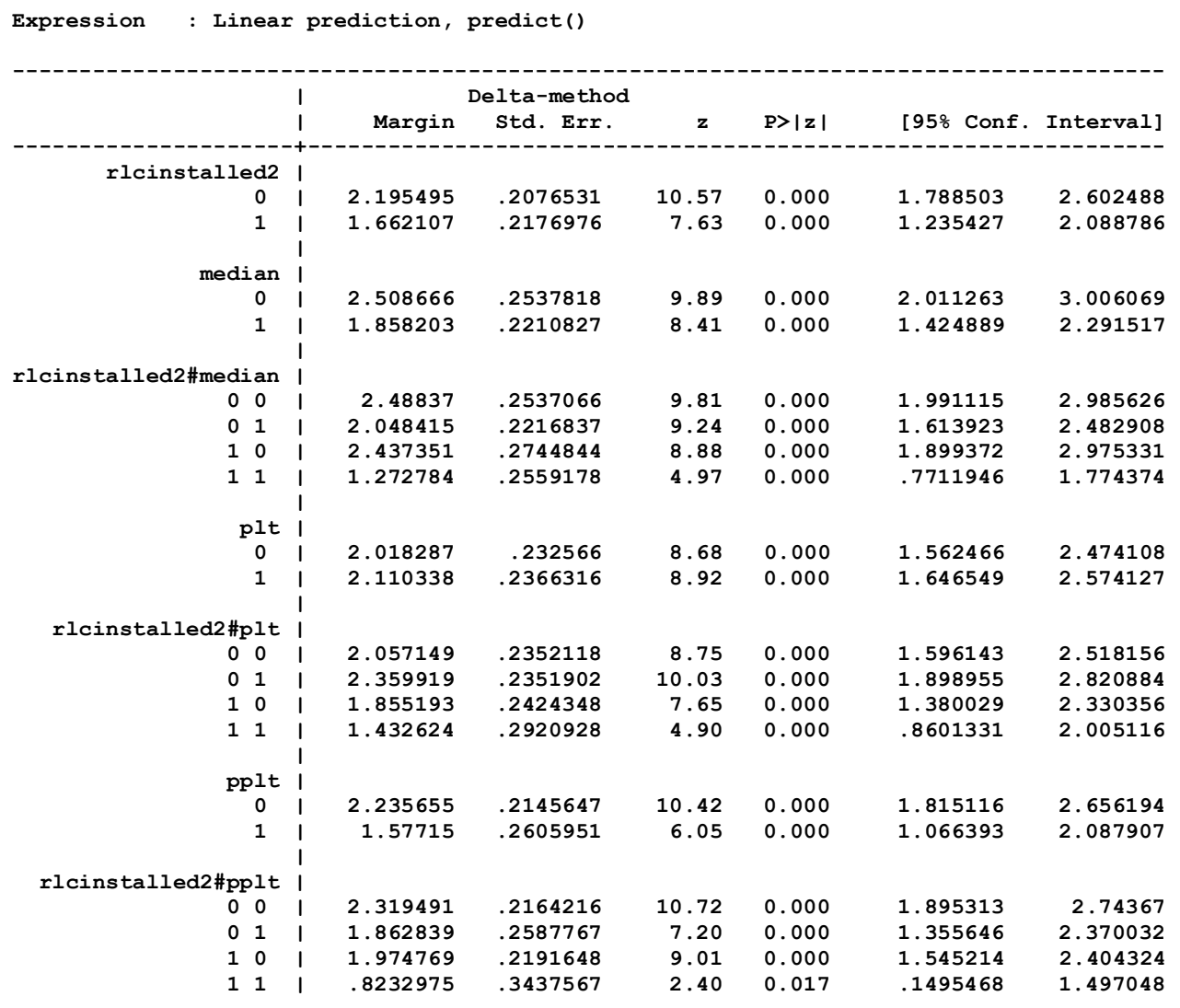

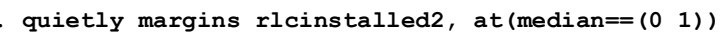

- marginsplot, recastci(rarea) ciopts (color(*.3))

Variables that uniquely identify margins: median rlcinstalled2

- margins, dydx(rlcinstalled2) at(median==( $\left.\left.\begin{array}{ll}0 & 1\end{array}\right)\right)$

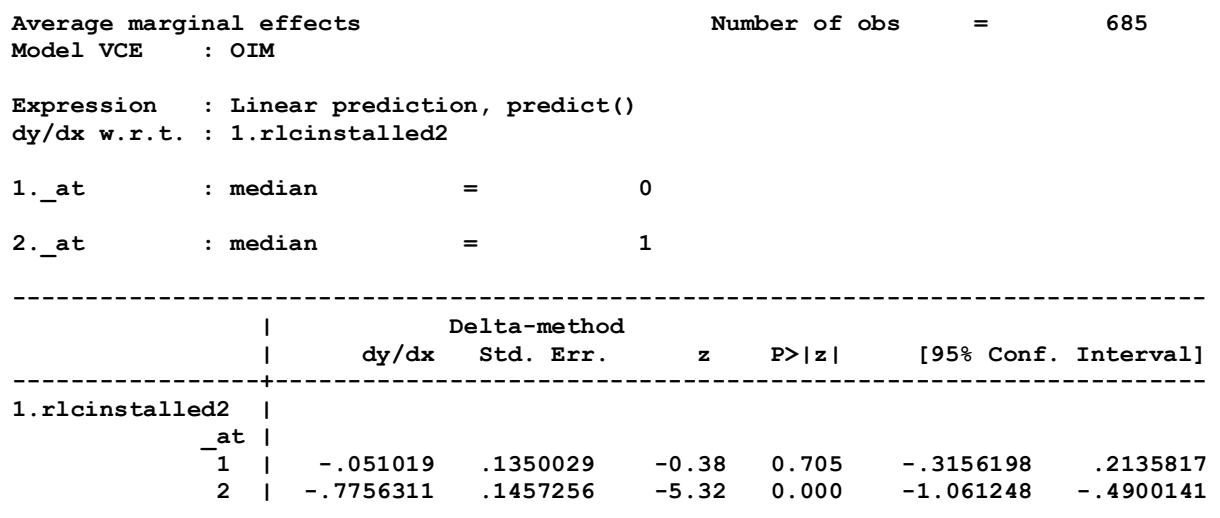

Note: $d y / d x$ for factor levels is the discrete change from the base level.

end of do-file

. do "C: \Users \Amal\AppData\Local\Temp\STD04000000. tmp"

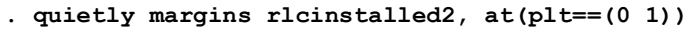

- marginsplot, recastci(rarea) ciopts(color(*.3)) 


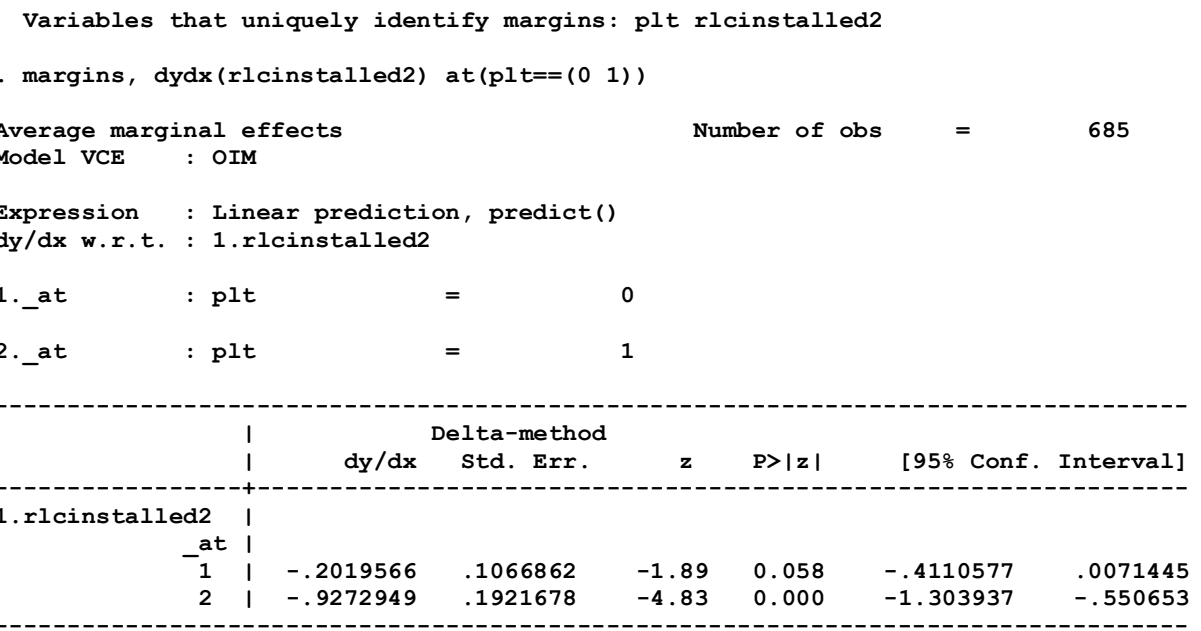

Note: dy/dx for factor levels is the discrete change from the base level.

end of do-file

. do "C: \Users \Amal\AppData\Local\Temp\STD04000000.tmp"

- quietly margins rlcinstalled2, at(pplt==(l 1 1))

- marginsplot, recastci (rarea) ciopts (color(*.3))

Variables that uniquely identify margins: pplt rlcinstalled2

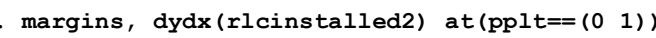

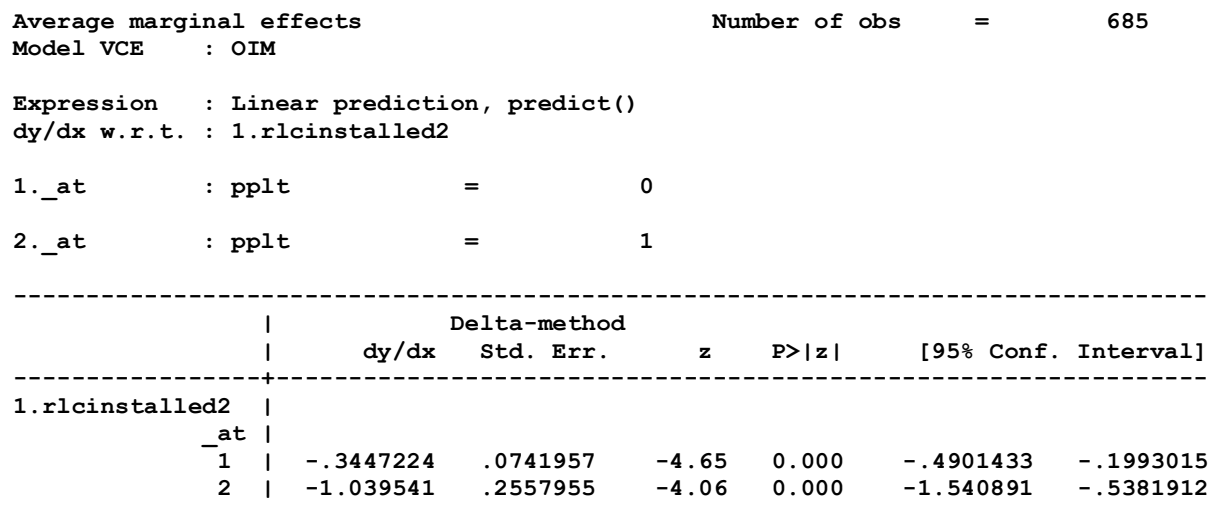

Note: $d y / d x$ for factor levels is the discrete change from the base level. 


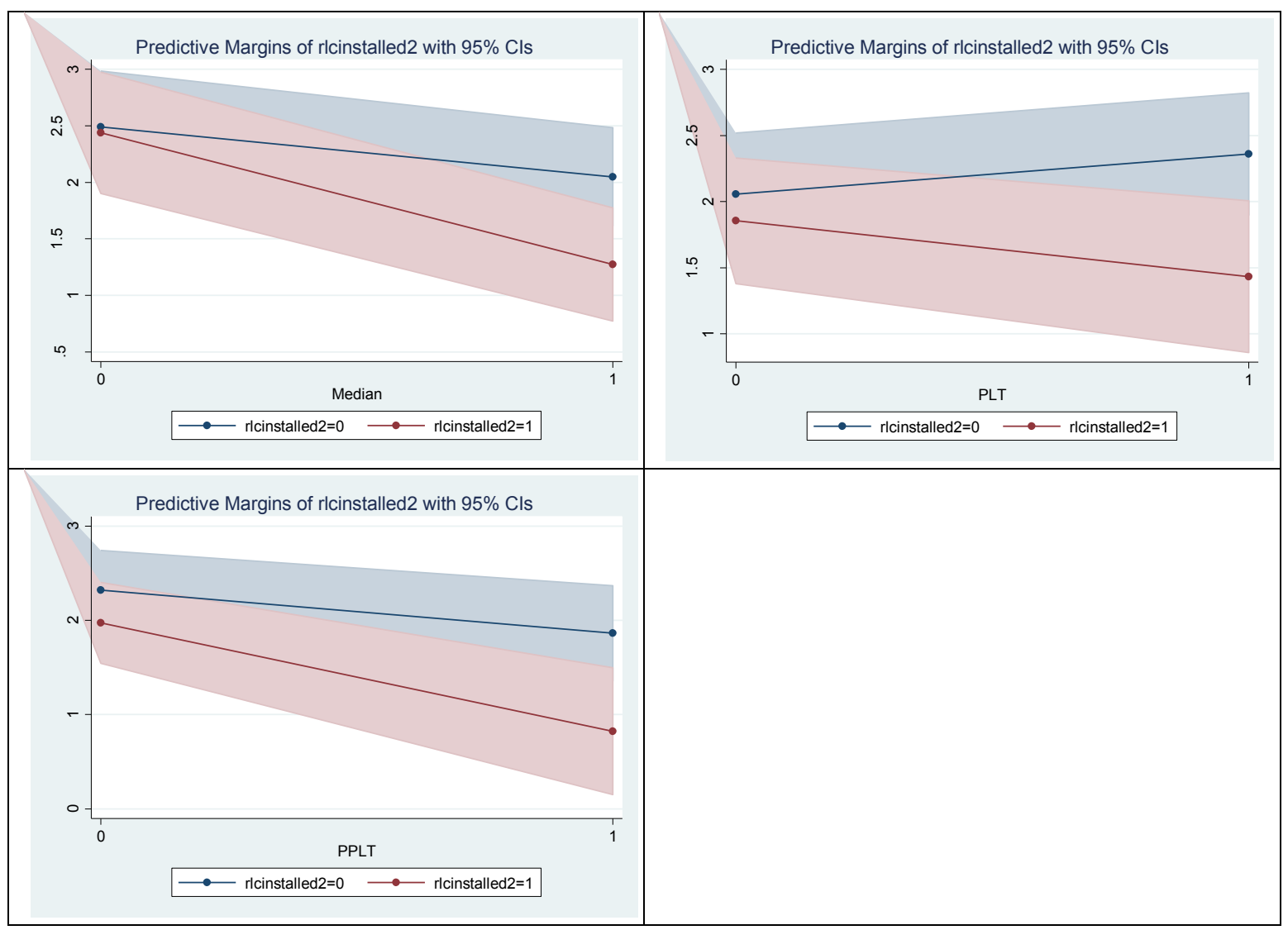

- xtnbreg turningmovement c.lnaadt i.rlcinstalled2 i.median i.channelization i.thrulanes i.lef $>$ tturn i.plt i.fplt i.pplt i.speed1 i.speed2 rlcinstalled2\#\#median rlcinstalled2\#\#leftturn rlc $>$ installed2\#\#thrulanes rlcinstalled2\#\#fplt rlcinstalled2\#\#plt rlcinstalled2\#\#pplt , irr re

Random-effects negative binomial regression Group variable: id

Random effects u_i Beta

Log likelihood $=-1293.8167$
Number of obs $=685$ Number of groups $=48$

Obs per group: $\begin{array}{lr}\min = & 14 \\ \operatorname{avg}= & 14.3 \\ \max = & 15\end{array}$

Wald $\operatorname{chi2}(27)=88.68$ Prob $>$ chi2 $=0.0000$

\begin{tabular}{|c|c|c|c|c|c|c|c|}
\hline turningmovement & 1 & IRR & Std. Err. & $\mathbf{z}$ & $P>|z|$ & [95\% Conf. & Interval] \\
\hline lnaadt & 1 & 1.442173 & .2118003 & 2.49 & 0.013 & 1.081454 & 1.923208 \\
\hline 1.rlcinstalled2 & 1 & 2.335753 & .9718715 & 2.04 & 0.041 & 1.033368 & 5.279576 \\
\hline 1.median & 1 & .5473002 & .1453215 & -2.27 & 0.023 & .3252452 & .9209589 \\
\hline 1. channelization & i & 1.563749 & .382381 & 1.83 & 0.067 & .9683317 & 2.525283 \\
\hline \multicolumn{8}{|c|}{ thrulanes } \\
\hline 2 & 1 & .56305 & .2040175 & -1.59 & 0.113 & .2767704 & 1.145445 \\
\hline 3 & 1 & .5672855 & .2505551 & -1.28 & 0.199 & .2386977 & 1.348202 \\
\hline 4 & 1 & 1.374543 & .7373458 & 0.59 & 0.553 & .4803398 & 3.9334 \\
\hline \multicolumn{8}{|c|}{ leftturn | } \\
\hline 1 & i & .7322197 & .1699302 & -1.34 & 0.179 & .4646206 & 1.153943 \\
\hline 2 & 1 & .9079109 & .3458032 & -0.25 & 0.800 & .4303674 & 1.915345 \\
\hline 1.plt & 1 & 1.122025 & .2994075 & 0.43 & 0.666 & .6650639 & 1.892963 \\
\hline 1.fplt & i & 1. 319512 & .3522562 & 1.04 & 0.299 & .7819468 & 2.226639 \\
\hline $1 \cdot \mathrm{pplt}$ & 1 & 2.568731 & .6685229 & 3.62 & 0.000 & 1.542375 & 4.278065 \\
\hline speed1 & 1 & & & & & & \\
\hline 50 & 1 & .8610169 & .2977071 & -0.43 & 0.665 & .4372184 & 1.695606 \\
\hline 60 & 1 & .7192228 & .2168036 & -1.09 & 0.274 & .3983602 & 1.298527 \\
\hline
\end{tabular}




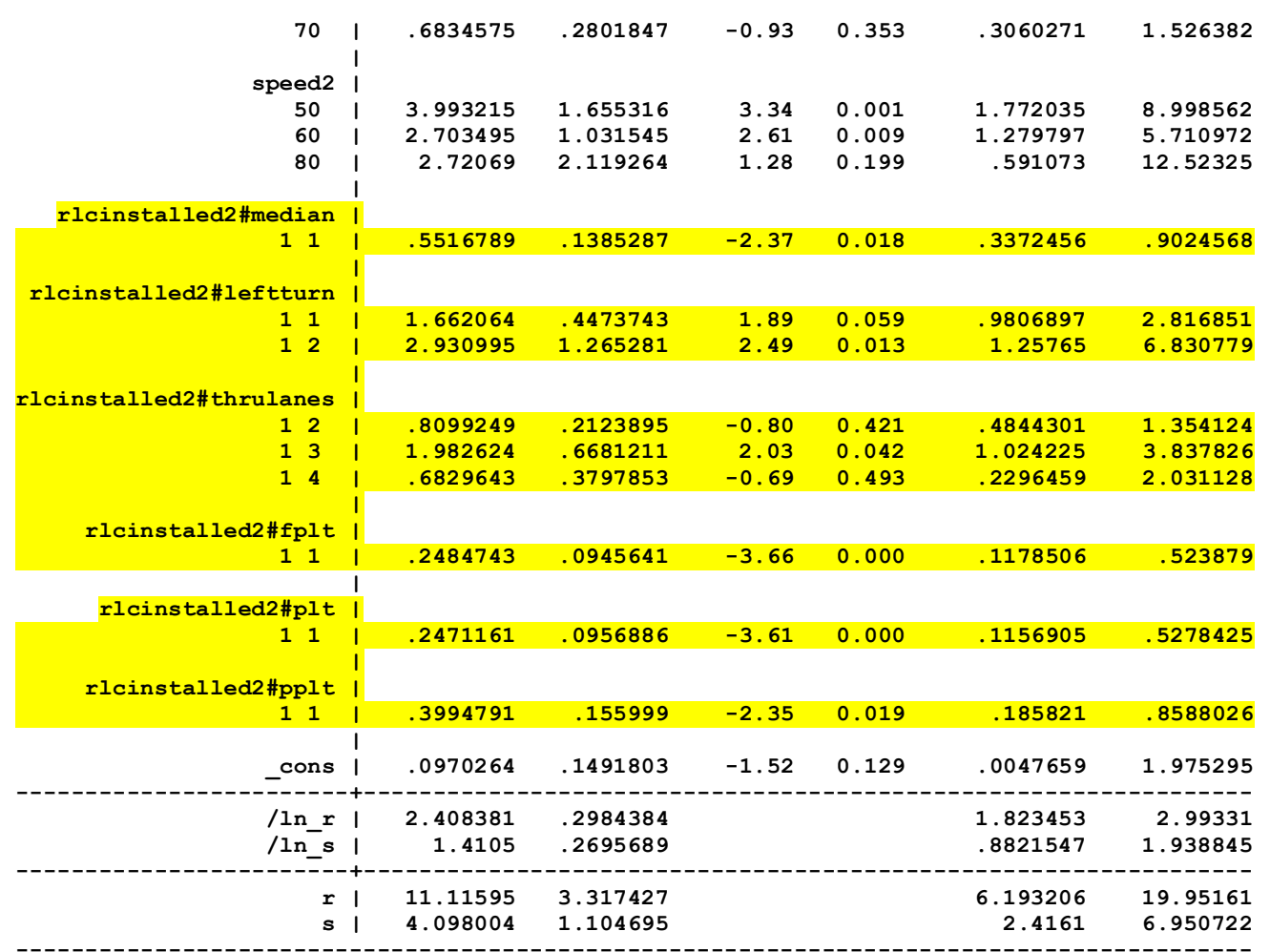

LR test vs. pooled: $\operatorname{chibar2}(01)=147.32 \quad$ Prob $>=\operatorname{chibar} 2=0.000$

- margins rlcinstalled2

Predictive margins

Model VCE : OIM

Number of obs $\quad=\quad 685$

Expression : Linear prediction, predict()

\begin{tabular}{|c|c|c|c|c|c|c|c|}
\hline & l & Margin & $\begin{array}{c}\text { Delta-method } \\
\text { Std. Err. }\end{array}$ & z & $P>|z|$ & [95\% Conf. & Interval] \\
\hline \multicolumn{8}{|c|}{ rlcinstalled2 | } \\
\hline $\begin{array}{l}0 \\
1\end{array}$ & l & $\begin{array}{l}1.888161 \\
1.293592\end{array}$ & $\begin{array}{r}.191952 \\
.2200469\end{array}$ & $\begin{array}{l}9.84 \\
5.88\end{array}$ & $\begin{array}{l}0.000 \\
0.000\end{array}$ & $\begin{array}{l}1.511942 \\
.8623078\end{array}$ & $\begin{array}{r}2.26438 \\
1.724876\end{array}$ \\
\hline
\end{tabular}

* *margins r.rlcinstalled2 ... same results as below:

margins, dydx(rlcinstalled2)

Average marginal effects $\quad$ Number of obs $=685$

Model VCE : OIM

Expression : Linear prediction, predict()

dy/dx w.r.t. : 1.rlcinstalled2

\begin{tabular}{|c|c|c|c|c|c|c|}
\hline & I & Delta-methoc & & & & \\
\hline & $d y / d x$ & Std. Err. & $\mathbf{z}$ & $P>|z|$ & [95\% Conf. & Interval] \\
\hline 1. rlcinstalled2 & -.5945695 & .1303914 & -4.56 & 0.000 & -.850132 & -.339007 \\
\hline
\end{tabular}

Note: $d y / d x$ for factor levels is the discrete change from the base level.

- margins rlcinstalled2\#median rlcinstalled2\#leftturn rlcinstalled2\#thrulanes rlcinstalled2\#\#pl

$>$ t rlcinstalled2\#\#fplt rlcinstalled2\#\#pplt

Predictive margins

Number of obs $\quad=\quad 685$

Model VCE : OIM

Expression : Linear prediction, predict() 


\begin{tabular}{|c|c|c|c|c|c|c|c|}
\hline & । & Margin & Std. Err. & $\mathbf{z}$ & $P>|z|$ & [95\% Conf & Interval] \\
\hline \multicolumn{8}{|l|}{ rlcinstalled2\#median } \\
\hline 00 & I & 2.289413 & .2614261 & 8.76 & 0.000 & 1.777027 & 2.801799 \\
\hline 01 & i & 1.686655 & .2111804 & 7.99 & 0.000 & 1.272749 & 2.100561 \\
\hline 10 & I & 2.090791 & .2884539 & 7.25 & 0.000 & 1.525432 & 2.65615 \\
\hline 11 & i & .893244 & .2691279 & 3.32 & 0.001 & .365763 & 1.420725 \\
\hline & I & & & & & & \\
\hline \multicolumn{8}{|l|}{ rlcinstalled2\#leftturn } \\
\hline 00 & i & 2.06 & .2352117 & 8.76 & 0.000 & 1.598994 & 2.521007 \\
\hline 01 & 1 & 1.748326 & .2199342 & 7.95 & 0.000 & 1.317262 & 2.179389 \\
\hline 02 & I & 1.963391 & .3738698 & 5.25 & 0.000 & 1.23062 & 2.696162 \\
\hline 10 & i & 1.10894 & .3252961 & 3.41 & 0.001 & .4713712 & 1.746509 \\
\hline 11 & i & 1.305325 & .2362809 & 5.52 & 0.000 & .8422234 & 1.768428 \\
\hline 12 & i & 2.087673 & .441207 & 4.73 & 0.000 & 1.222923 & 2.952422 \\
\hline & i & & & & & & \\
\hline \multicolumn{8}{|l|}{ rlcinstalled2\#thrulanes } \\
\hline 01 & 1 & 2.356646 & .3791596 & 6.22 & 0.000 & 1.613506 & 3.099785 \\
\hline 02 & i & 1.782259 & .2083433 & 8.55 & 0.000 & 1.373913 & 2.190604 \\
\hline 03 & i & 1.789753 & .2918556 & 6.13 & 0.000 & 1.217727 & 2.361779 \\
\hline 04 & i & 2.674767 & .4169032 & 6.42 & 0.000 & 1.857652 & 3.491882 \\
\hline 11 & i & 1.761308 & .4454177 & 3.95 & 0.000 & .8883052 & 2.63431 \\
\hline 12 & i & .9761072 & .2534439 & 3.85 & 0.000 & .4793663 & 1.472848 \\
\hline 13 & i & 1.878836 & .285391 & 6.58 & 0.000 & 1.31948 & 2.438192 \\
\hline 14 & I & 1.698116 & .5425648 & 3.13 & 0.002 & .6347091 & 2.761524 \\
\hline & 1 & & & & & & \\
\hline \multicolumn{8}{|c|}{ rlcinstalled2 } \\
\hline 0 & i & 1.888161 & .191952 & 9.84 & 0.000 & 1.511942 & 2.26438 \\
\hline 1 & i & 1.293592 & .2200469 & 5.88 & 0.000 & .8623078 & 1.724876 \\
\hline & i & & & & & & \\
\hline \multicolumn{8}{|c|}{ plt I } \\
\hline 0 & I & 1.913221 & .2262493 & 8.46 & 0.000 & 1.469781 & 2.356662 \\
\hline 1 & i & 1.622253 & .2455011 & 6.61 & 0.000 & 1.141079 & 2.103426 \\
\hline & i & & & & & & \\
\hline \multicolumn{8}{|c|}{ rlcinstalled2\#plt | } \\
\hline 00 & i & 1.835552 & .2275525 & 8.07 & 0.000 & 1.389557 & 2.281547 \\
\hline 01 & i & 1.950687 & .240345 & 8.12 & 0.000 & 1.47962 & 2.421755 \\
\hline 10 & 1 & 1.87973 & .2417407 & 7.78 & 0.000 & 1.405927 & 2.353533 \\
\hline 11 & i & .5969681 & .3795347 & 1.57 & 0.116 & -.1469063 & 1.340843 \\
\hline & i & & & & & & \\
\hline \multicolumn{8}{|c|}{ fplt I } \\
\hline 0 & I & 1.86038 & .2168101 & 8.58 & 0.000 & 1.43544 & 2.28532 \\
\hline 1 & i & 1.73313 & .2560243 & 6.77 & 0.000 & 1.231332 & 2.234929 \\
\hline & 1 & & & & & & \\
\hline \multicolumn{8}{|l|}{ rlcinstalled2\#fplt | } \\
\hline 00 & i & 1.785352 & .2171952 & 8.22 & 0.000 & 1.359657 & 2.211046 \\
\hline 01 & 1 & 2.062614 & .253301 & 8.14 & 0.000 & 1.566153 & 2.559075 \\
\hline 10 & i & 1.707094 & .2311639 & 7.38 & 0.000 & 1.254021 & 2.160167 \\
\hline 11 & I & .5919404 & .400299 & 1.48 & 0.139 & -.1926313 & 1.376512 \\
\hline \multirow{2}{*}{\multicolumn{8}{|c|}{ pplt }} \\
\hline & & & & & & & \\
\hline 0 & 1 & 1.601453 & .2046626 & 7.82 & 0.000 & 1.200321 & 2.002584 \\
\hline 1 & i & 2.278294 & .2699137 & 8.44 & 0.000 & 1.749272 & 2.807315 \\
\hline & 1 & & & & & & \\
\hline \multicolumn{8}{|l|}{ rlcinstalled2\#pplt | } \\
\hline 00 & $i$ & 1.631994 & .2066691 & 7.90 & 0.000 & 1.22693 & 2.037058 \\
\hline 01 & 1 & 2.575406 & .2654195 & 9.70 & 0.000 & 2.055193 & 3.095619 \\
\hline 10 & 1 & 1.286581 & .2172105 & 5.92 & 0.000 & .8608567 & 1.712306 \\
\hline 11 & i & 1.312399 & .4356417 & 3.01 & 0.003 & .4585574 & 2.166242 \\
\hline
\end{tabular}

- quietly margins rlcinstalled2, at(median==( $\left.\left.\begin{array}{ll}0 & 1\end{array}\right)\right)$

- marginsplot, recastci (rarea) ciopts (color(*.3))

Variables that uniquely identify margins: median rlcinstalled2

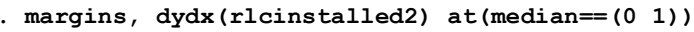

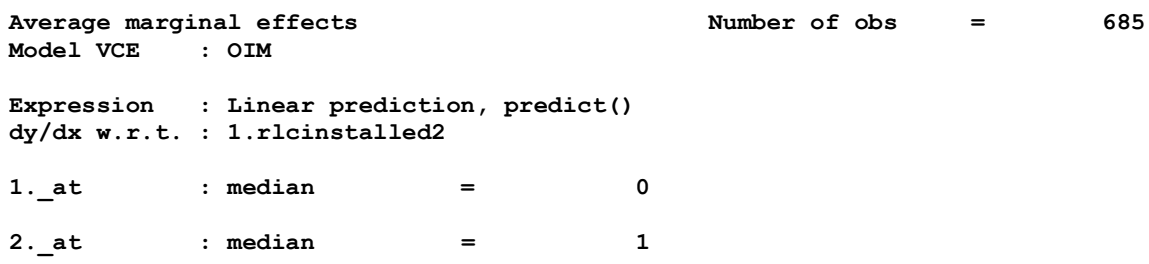




\begin{tabular}{|c|c|c|c|c|c|c|c|}
\hline & $\begin{array}{l}1 \\
1\end{array}$ & $d y / d x$ & $\begin{array}{l}\text { Delta-method } \\
\text { Std. Err. }\end{array}$ & $\mathbf{z}$ & $\mathrm{P}>|\mathbf{z}|$ & [95\% Conf. & Interval] \\
\hline rlcinstalled2 & 1 & & & & & & \\
\hline _at & 1 & & & & & & \\
\hline 1 & 1 & -.1986223 & .152913 & -1.30 & 0.194 & -.4983262 & .1010816 \\
\hline 2 & 1 & -.7934114 & .1867533 & -4.25 & 0.000 & -1.159441 & -.4273816 \\
\hline
\end{tabular}

Note: $d y / d x$ for factor levels is the discrete change from the base level.

end of do-file

. do "C: \Users \Amal\AppData\Local\Temp\STD04000000.tmp"

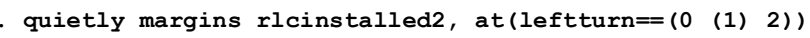

marginsplot, recastci(rarea) ciopts (color(*.3))

Variables that uniquely identify margins: leftturn rlcinstalled2

margins, dydx(rlcinstalled2) at(leftturn==( $\left.\begin{array}{lll}0 & \text { (1) } 2)\end{array}\right)$

Average marginal effects $\quad$ Number of obs $=685$

Model VCE : OIM

Expression : Linear prediction, predict()

dy/dx w.r.t. : 1.rlcinstalled2

1._at

: lefturn $\quad=\quad 0$

2._at : lefturn $\quad=\quad 1$

3. at : leftturn $\quad=\quad 2$

\begin{tabular}{|c|c|c|c|c|c|c|c|}
\hline & 1 & $d y / d x$ & $\begin{array}{c}\text { Delta-method } \\
\text { Std. Err. }\end{array}$ & $\mathbf{z}$ & $P>|z|$ & [95\% Conf. & Interval] \\
\hline rlcinstalled2 & 1 & & & & & & \\
\hline at & 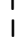 & & & & & & \\
\hline 1 & $i$ & -.9510603 & .2574827 & -3.69 & 0.000 & -1.455717 & -.4464034 \\
\hline 2 & i & -.4430001 & .1308479 & -3.39 & 0.001 & -.6994572 & -.1865429 \\
\hline 3 & i & .1242816 & .3170181 & 0.39 & 0.695 & -.4970625 & .7456256 \\
\hline
\end{tabular}

Note: dy/dx for factor levels is the discrete change from the base level.

end of do-file

. do "C: \Users \Amal\AppData\Local\Temp\STD04000000.tmp"

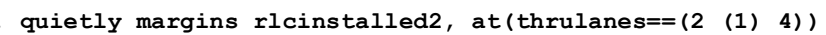

marginsplot, recastci(rarea) ciopts (color(*.3))

Variables that uniquely identify margins: thrulanes rlcinstalled2

margins, dydx(rlcinstalled2) at(thrulanes==(1 (1) 4))

Average marginal effects $\quad$ Number of obs $=685$

Model VCE : OIM

Expression : Linear prediction, predict()

$\mathrm{dy} / \mathrm{dx}$ w.r.t. : 1.rlcinstalled2

1._at : thrulanes $\quad=\quad 1$

2._at : thrulanes $\quad=\quad 2$

3. at : thrulanes = 3

4. at : thrulanes $=4$

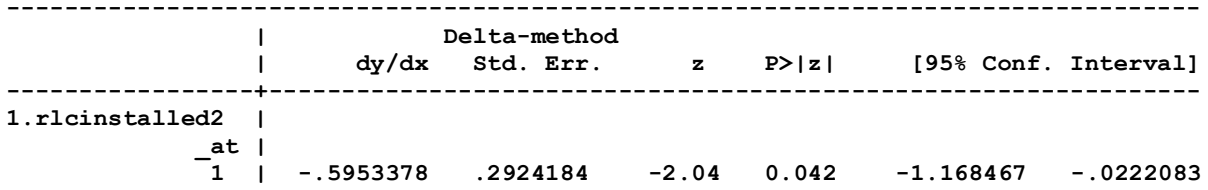




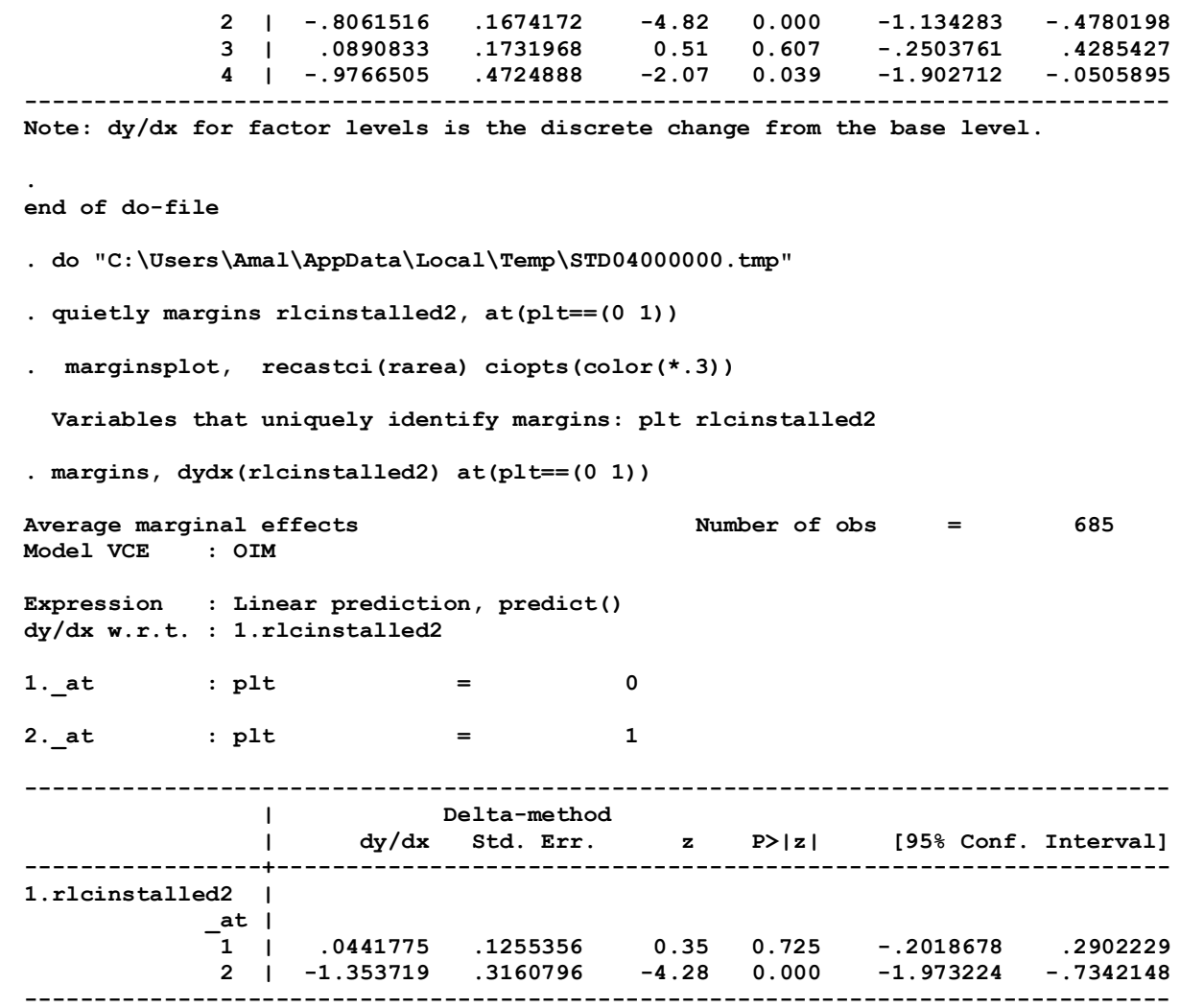

Note: $\mathrm{dy} / \mathrm{dx}$ for factor levels is the discrete change from the base level.

end of do-file

. do "C: \Users \Amal\AppData\Local\Temp\STD04000000 .tmp"

- quietly margins rlcinstalled2, at (fplt $\left.==\left(\begin{array}{ll}0 & 1\end{array}\right)\right)$

. marginsplot, recastci(rarea) ciopts(color(*.3))

Variables that uniquely identify margins: fplt rlcinstalled2

- margins, dydx(rlcinstalled2) at (fplt $\left.==\left(\begin{array}{ll}0 & 1\end{array}\right)\right)$

Average marginal effects $\quad$ Number of obs $=685$

Model VCE : OIM

Expression : Linear prediction, predict()

dy/dx w.r.t. : 1.rlcinstalled2

$\begin{array}{llll}\text { 1._at } & \text { : fplt } & = & 0 \\ \text { 2._at } & \text { : fplt } & = & 1\end{array}$

\begin{tabular}{|c|c|c|c|c|c|c|c|}
\hline & I & & Delta-method & & & & \\
\hline & I & $d y / d x$ & Std. Err. & $\mathbf{z}$ & $P>|z|$ & [95\% Conf. & Interval] \\
\hline \multirow{2}{*}{\multicolumn{8}{|c|}{$\begin{array}{r}\text { 1. rlcinstalled2 } \\
\text { at }\end{array}$}} \\
\hline & & & & & & & \\
\hline 1 & I & -.0782577 & .1197879 & -0.65 & 0.514 & -.3130377 & .1565224 \\
\hline 2 & 1 & -1.470674 & .3356136 & -4.38 & 0.000 & -2.128464 & -.812883 \\
\hline
\end{tabular}

Note: dy/dx for factor levels is the discrete change from the base level.

end of do-file

. do "C: \Users \Amal\AppData\Local\Temp\STD04000000. tmp"

. quietly margins rlcinstalled2, at $\left(p p l t==\left(\begin{array}{ll}0 & 1\end{array}\right)\right)$ 


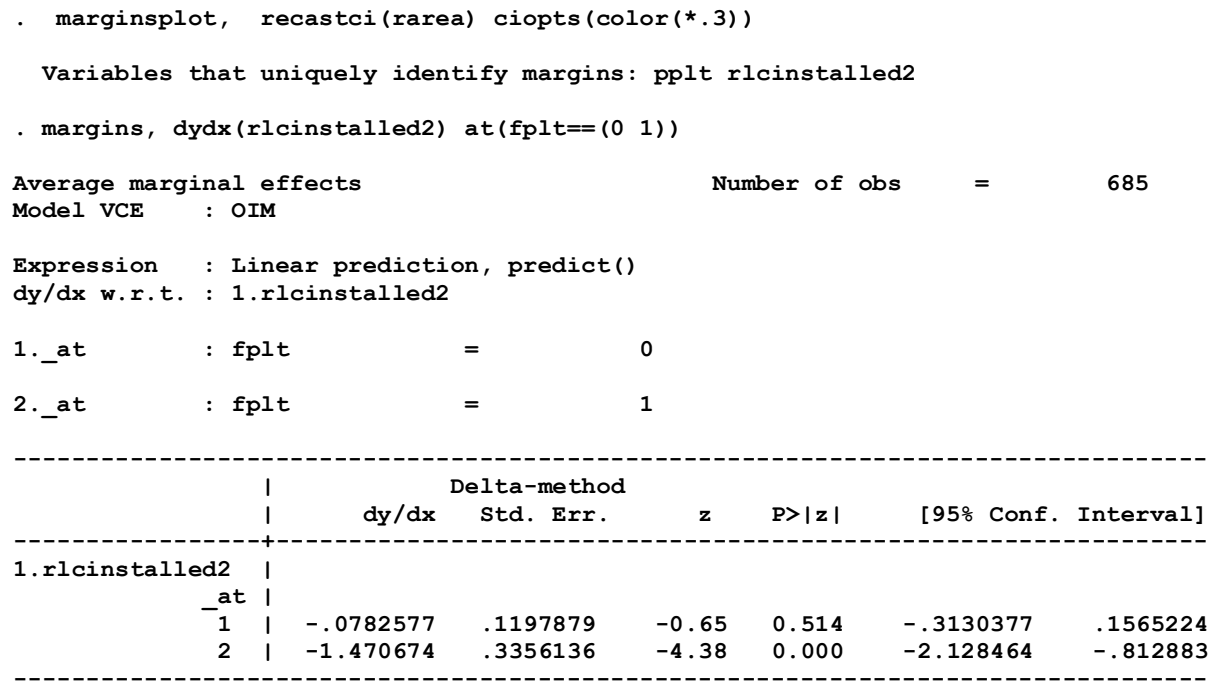

Note: $d y / d x$ for factor levels is the discrete change from the base level.

end of do-file

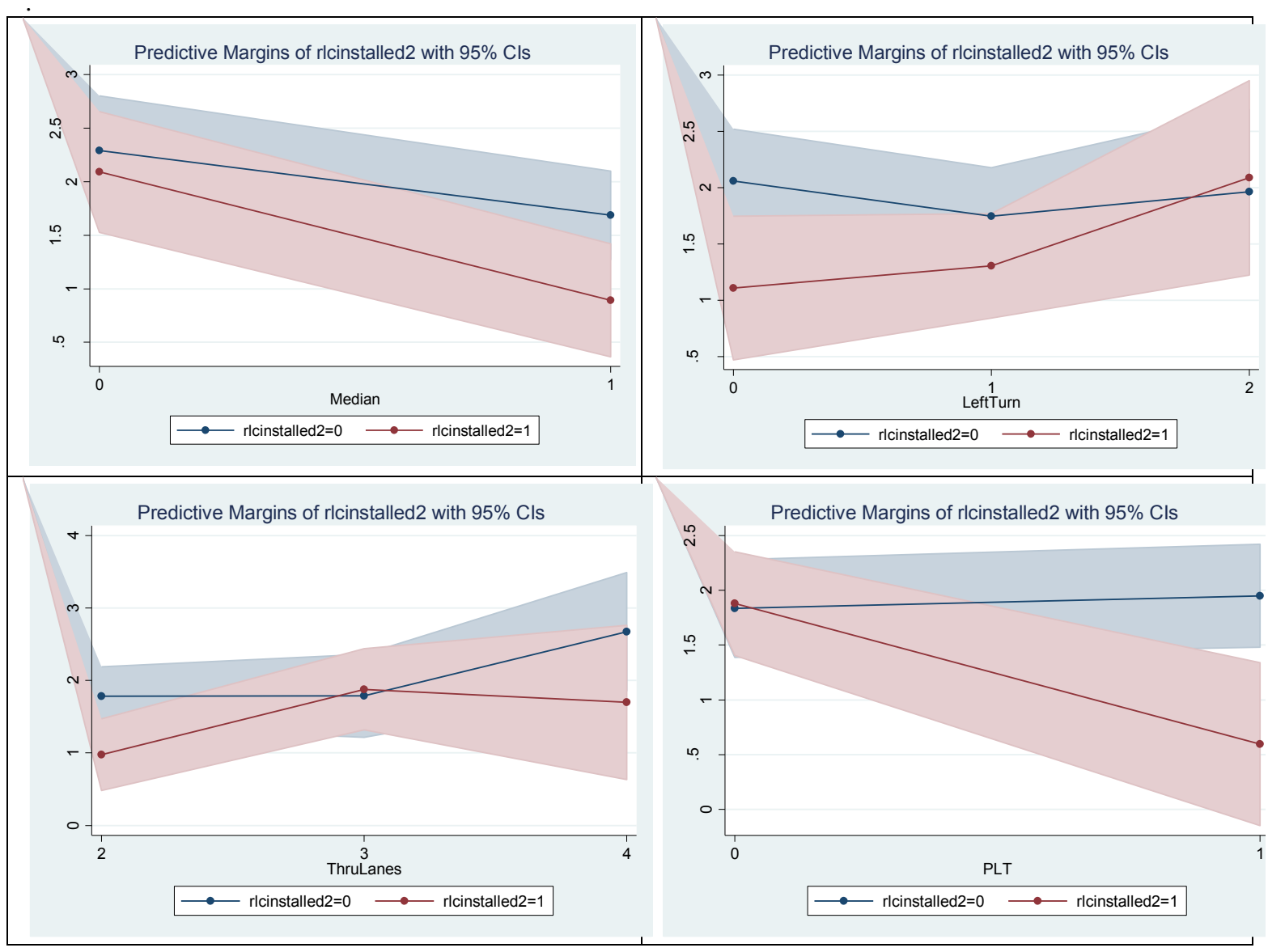




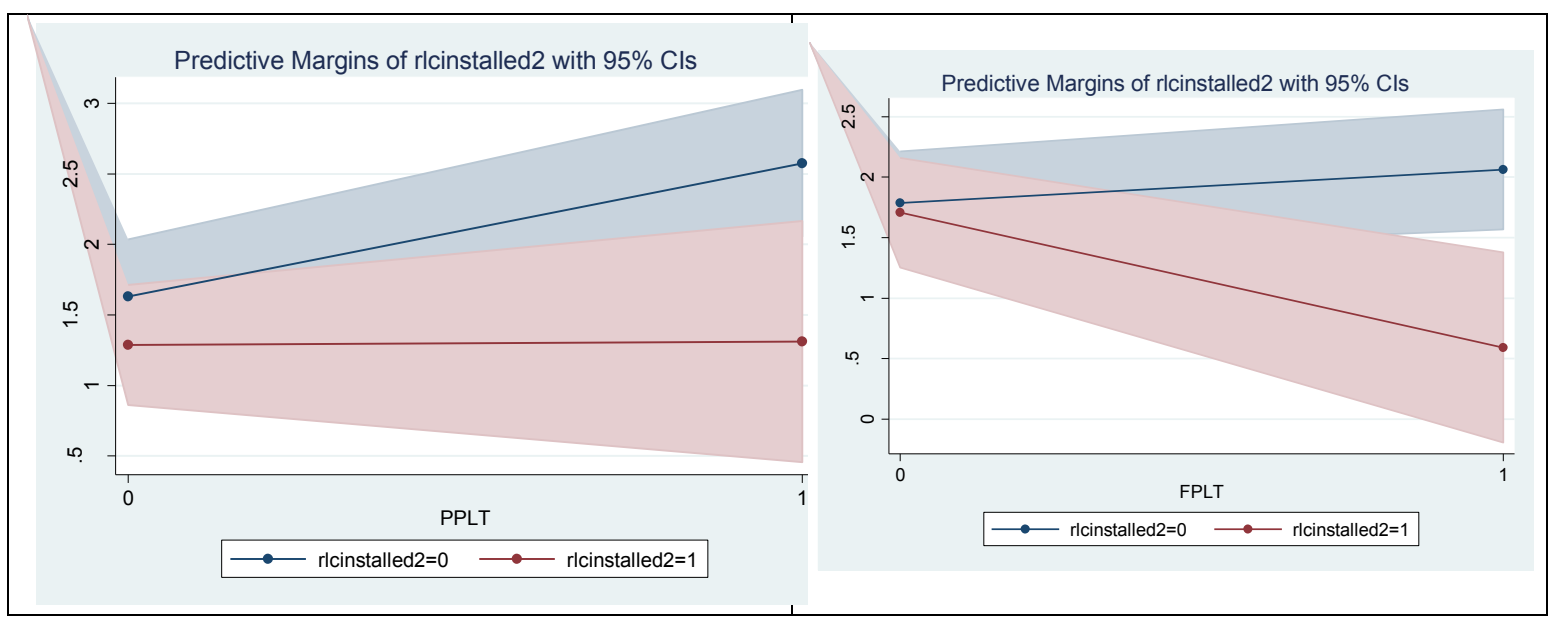

- xtnbreg sideswipe c.lnaadt i.rlcinstalled2 i.median i.channelization i.thrulanes $i . l e f t t u r n$ $>$ i.plt i.fplt i.pplt i.speed1 i.speed2 rlcinstalled2\#\#median rlcinstalled2\#\#plt, irr re

Fitting negative binomial (constant dispersion) model:

Random-effects negative binomial regression

Group variable: id

Number of obs $\quad=\quad 685$

Random effects $u_{-} i \sim$ Beta

Obs per group:

$\begin{array}{rrr}\min & = & 14 \\ \operatorname{avg}= & & 14.3 \\ \max & = & \\ & = & 86.00 \\ & = & 0.0000\end{array}$

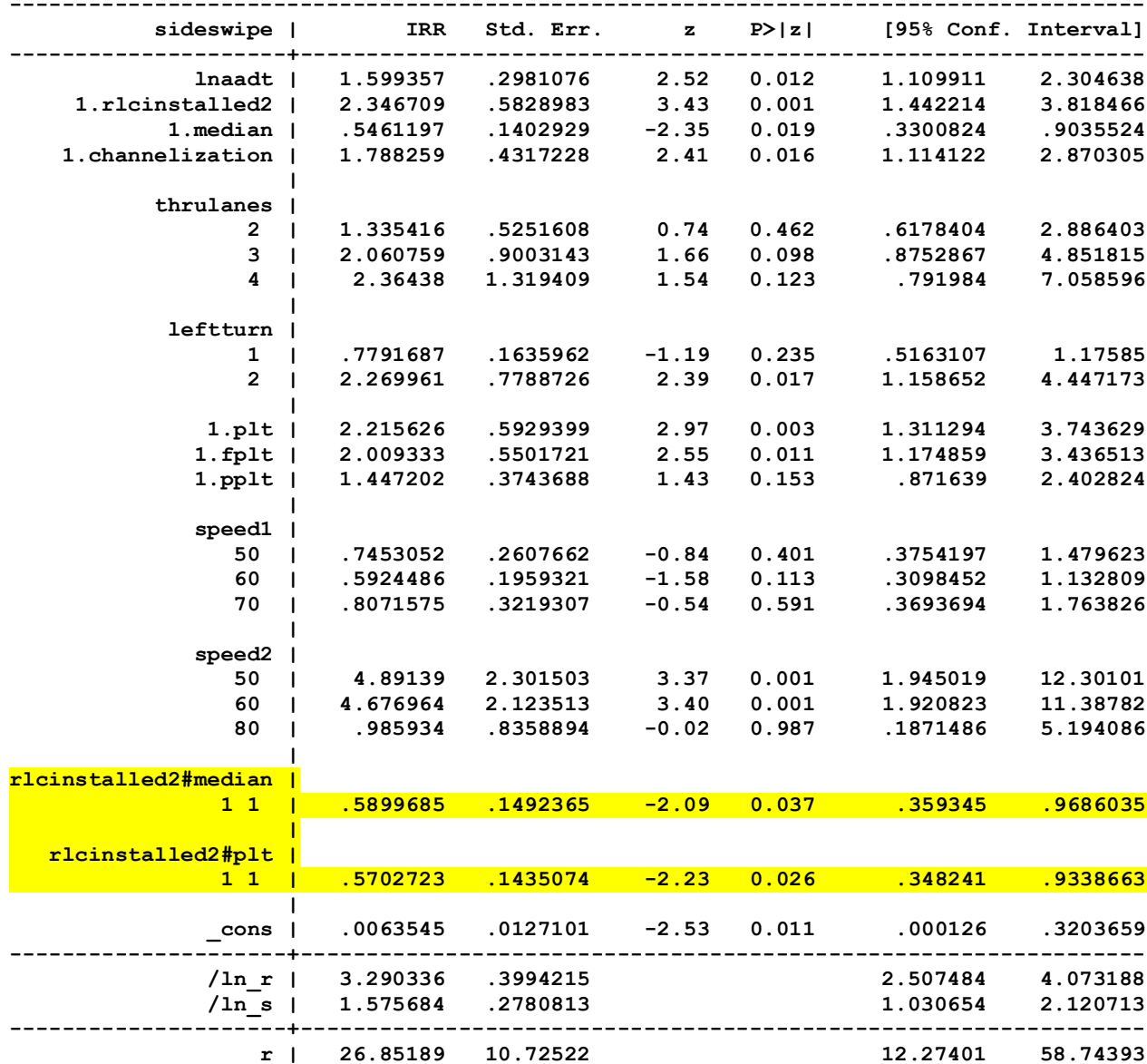


s I $4.834046 \quad 1.344258$

LR test vs. pooled: chibar2 $(01)=88.05$

- margins rlcinstalled2

Predictive margins

Model VCE : OIM

Number of obs $\quad=\quad 685$

2. 8029

8.337081

Expression : Linear prediction, predict()
Prob >= chibar2 $=0.000$

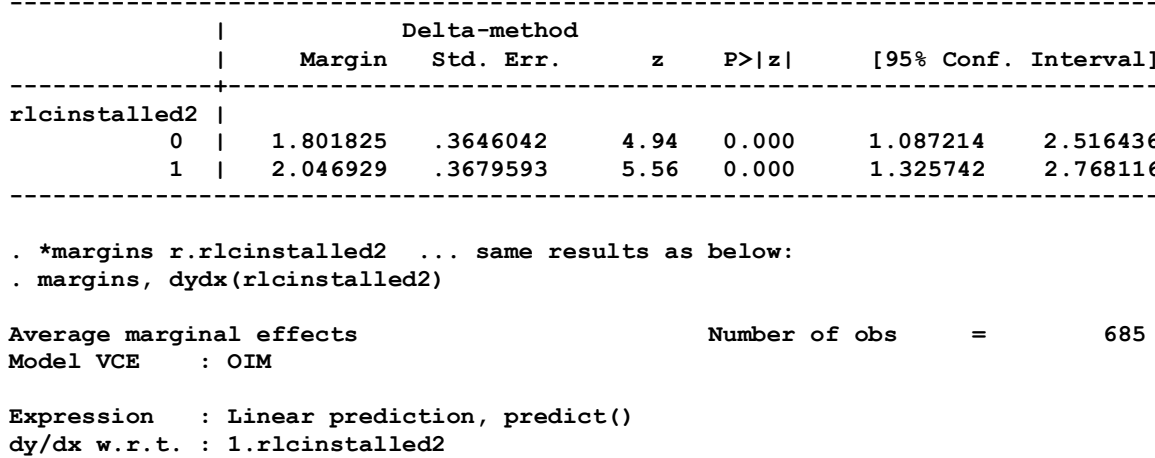

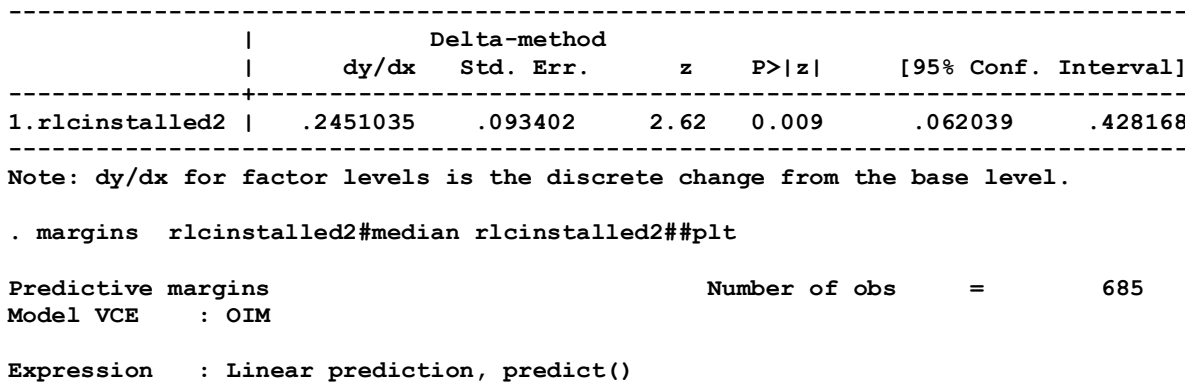

Expression : Linear prediction, predict()

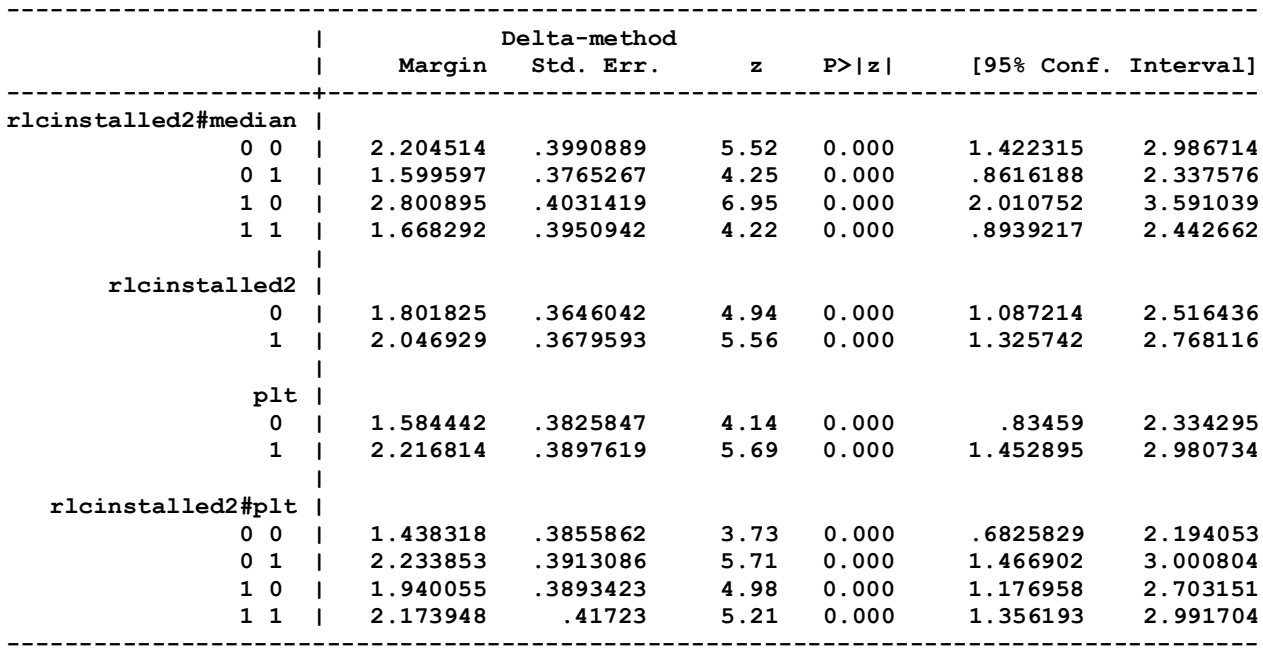

quietly margins rlcinstalled2, at(median==( $\left.\left.\begin{array}{ll}0 & 1\end{array}\right)\right)$

marginsplot, recastci (rarea) ciopts (color(*.3))

Variables that uniquely identify margins: median rlcinstalled2

margins, dydx(rlcinstalled2) at(median==( $\left.\left.\begin{array}{ll}0 & 1\end{array}\right)\right)$

Average marginal effects

Number of obs

685 


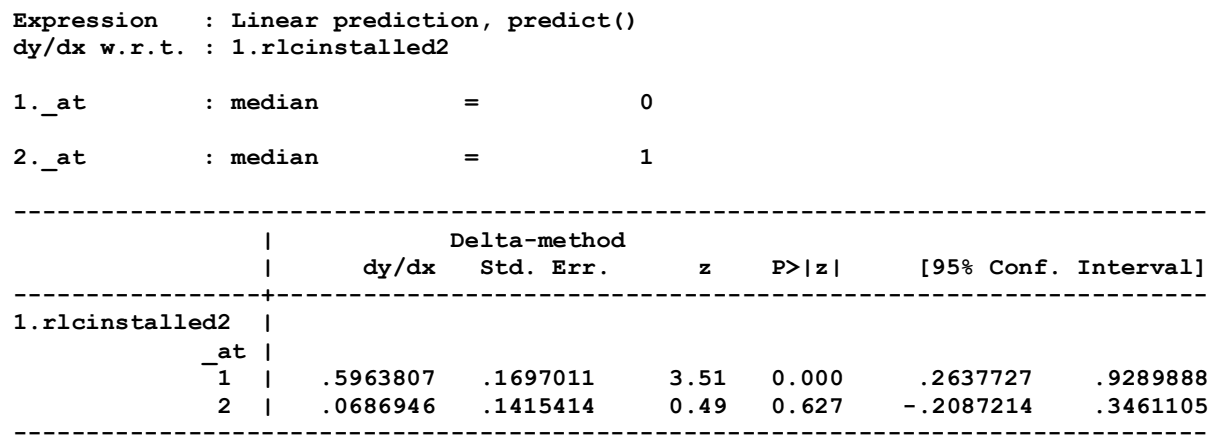

Note: $d y / d x$ for factor levels is the discrete change from the base level.

end of do-file

. do "C: \Users\Amal\AppData\Local\Temp\STD04000000.tmp"

- quietly margins rlcinstalled2, $\operatorname{at}\left(\mathrm{plt}==\left(\begin{array}{ll}0 & 1\end{array}\right)\right)$

- marginsplot, recastci(rarea) ciopts (color(*.3))

Variables that uniquely identify margins: plt rlcinstalled2

- margins, $d y d x(r l c i n s t a l l e d 2)$ at $\left(p l t==\left(\begin{array}{ll}0 & 1\end{array}\right)\right)$

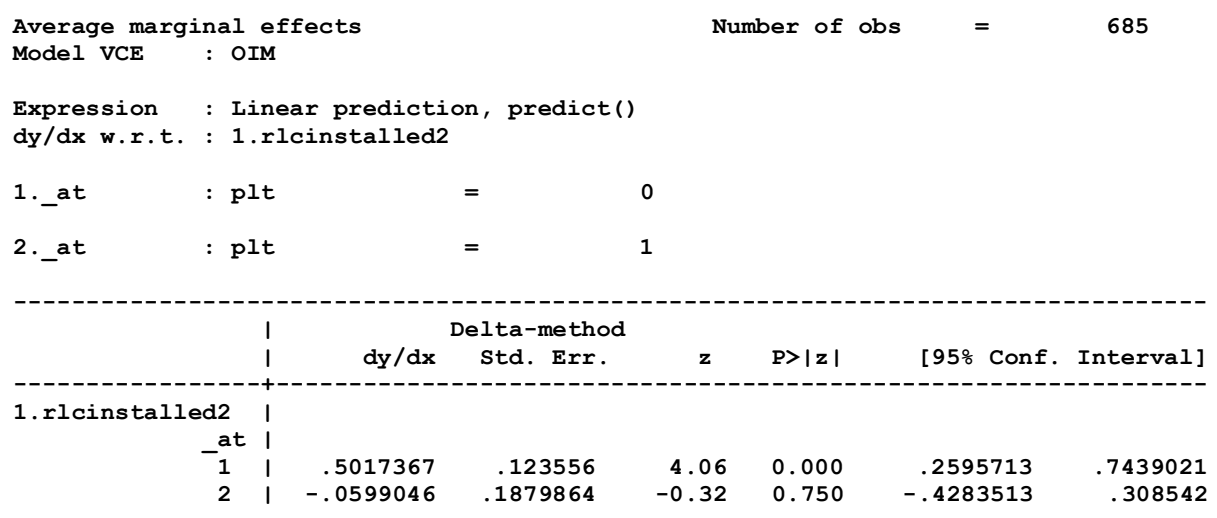

Note: dy/dx for factor levels is the discrete change from the base level.

end of do-file

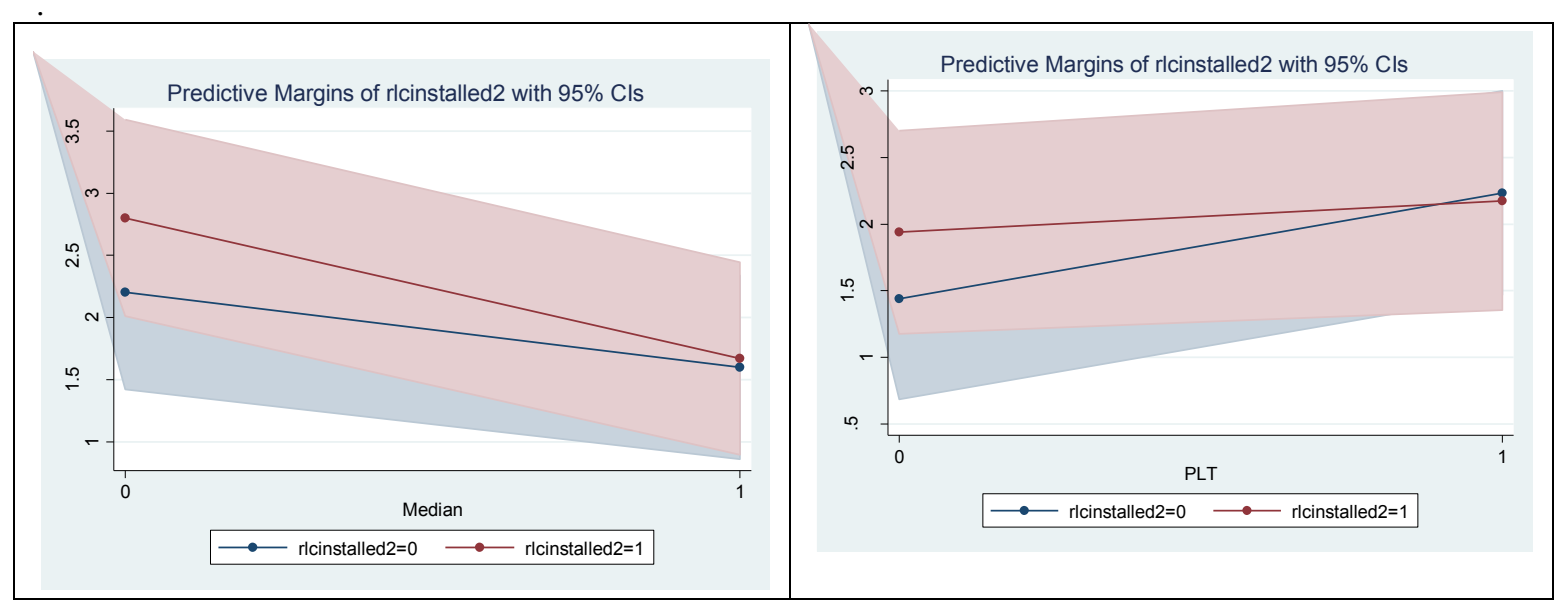




\section{Appendix I - Spillover Models}

\begin{tabular}{|c|c|c|c|c|c|}
\hline \multirow{2}{*}{$\begin{array}{c}\text { Collision Impact } \\
\text { Type } \\
\end{array}$} & $\begin{array}{c}\text { AAOC }^{\mathbf{a}} \\
\text { (Before } \\
\text { RLC) }\end{array}$ & $\begin{array}{c}\text { AAOC } \\
\text { (After } \\
\text { RLC) } \\
\end{array}$ & $\begin{array}{l}\text { ATT } \\
\text { Coef. }\end{array}$ & $\begin{array}{c}\text { Effect } \\
\text { without } \\
\text { RLC } \\
\text { (2) -(3) }\end{array}$ & $\begin{array}{l}\begin{array}{l}\text { Percent } \\
\text { Change }\end{array} \\
(2)-(4) /(4)\end{array}$ \\
\hline & (1) & (2) & (3) & (4) & (5) \\
\hline Total Collisions & 14.18 & 16.72 & -0.0289 & 16.75 & $-0.2 \%$ \\
\hline Rear end & 5.00 & 7.88 & 0.0158 & 7.86 & $0.2 \%$ \\
\hline Angle & 3.82 & 3.08 & -0.2676 & 3.35 & $-8.0 \%$ \\
\hline $\begin{array}{l}\text { Turning } \\
\text { movement }\end{array}$ & 3.03 & 2.69 & -0.2433 & 2.93 & $-8.3 \%$ \\
\hline Sideswipe & 1.35 & 1.96 & -0.1341 & 2.09 & $-6.4 \%$ \\
\hline
\end{tabular}

Table B - Impact of RLC in terms of percentage of collision reduction (XTNBREG estimates)

\begin{tabular}{|l|c|c|r|r|r|r|}
\hline $\begin{array}{l}\text { Collision impact } \\
\text { type }\end{array}$ & Coef. & $\begin{array}{l}\text { Std. } \\
\text { Err. }\end{array}$ & \multicolumn{1}{|c|}{$\mathbf{z}$} & P>chi2 & \multicolumn{2}{|l|}{$\begin{array}{l}\text { [95\% Conf. } \\
\text { Interval] }\end{array}$} \\
\hline \hline Total collisions & -0.0289 & 0.0432 & -0.67 & 0.5040 & -0.1136 & 0.0559 \\
\hline Rear end & 0.0158 & 0.0727 & 0.22 & 0.8280 & -0.1267 & 0.1583 \\
\hline Angle & -0.2676 & 0.1124 & -2.38 & 0.0170 & -0.4878 & -0.0474 \\
\hline $\begin{array}{l}\text { Turning } \\
\text { movement }\end{array}$ & -0.2433 & 0.0846 & -2.87 & 0.0040 & -0.4092 & -0.0774 \\
\hline Sideswipe & -0.1341 & 0.1162 & -1.15 & 0.2490 & -0.3619 & 0.0937 \\
\hline
\end{tabular}

Table C - Significant interactions from spillover models (XTNBREG)

\begin{tabular}{|c|c|c|c|c|c|}
\hline & Total & Rear-end & Angle & TM & Sideswipe \\
\hline Variables & \multicolumn{5}{|c|}{ Determining effects of significant interactions with the RLC } \\
\hline rlcinstalled2 & $V_{<1 \%}$ & 1 $<1 \%$ & $\sqrt{8 \%}$ & $v_{9 \%}$ & $V_{7 \%}$ \\
\hline median & $V_{24 \%}$ & & $\sqrt{51 \%}$ & $V_{45 \%}$ & $\sqrt{45 \%}$ \\
\hline channelization & 个 $27 \%$ & 个 $58 \%$ & & & \\
\hline leftturn & $\begin{array}{l}\text { - With } 1 \text { left-turn } \\
\text { lane } V_{23 \%} \\
\text { (significant) } \\
\text { - With } 2 \text { left-turn } \\
\text { lanes } V_{12 \%}\end{array}$ & $\begin{array}{l}\text { - With } 1 \text { left- } \\
\text { turn lane } \\
\downarrow_{31 \%} \\
\text { - With } 2 \text { left- } \\
\text { turn lanes } \\
V_{32 \%}\end{array}$ & & $\begin{array}{l}\text { - With } 1 \text { left-turn } \\
\text { lane } 66 \% \\
\text { - With } 2 \text { left-turn } \\
\text { lanes } 290 \% \\
\text { (significant) }\end{array}$ & \\
\hline plt & $\sqrt{49 \%}$ & & $\sqrt{50 \%}$ & $\sqrt{75 \%}$ & $\sqrt{47 \%}$ \\
\hline
\end{tabular}




\begin{tabular}{|c|c|c|c|}
\hline fplt & & & $V_{75 \%}$ \\
\hline pplt & & $V_{51 \%}$ & $V_{60 \%}$ \\
\hline \multicolumn{4}{|l|}{ speed1 } \\
\hline \multicolumn{4}{|l|}{ speed 2} \\
\hline \multirow[b]{4}{*}{ thrulanes } & $\begin{array}{l}\text { - With } 2 \text { thru } \\
\text { lanes }>1 \%\end{array}$ & & $\begin{array}{c}\text { - With } 2 \text { thru lanes } \\
\sqrt[2]{20 \%}\end{array}$ \\
\hline & $\begin{array}{l}\text { - With } 2 \text { thru } \\
\text { lanes }><1 \%\end{array}$ & & $\begin{array}{c}\text { - With } 3 \text { thru lanes } \\
198 \%\end{array}$ \\
\hline & $\begin{array}{l}\text { - With } 4 \text { thru } \\
\text { lanes } 40 \%\end{array}$ & & (significant) \\
\hline & (significant) & & $\begin{array}{c}\text { - With } 4 \text { thru lanes } \\
\sqrt[31 \%]{ }\end{array}$ \\
\hline
\end{tabular}

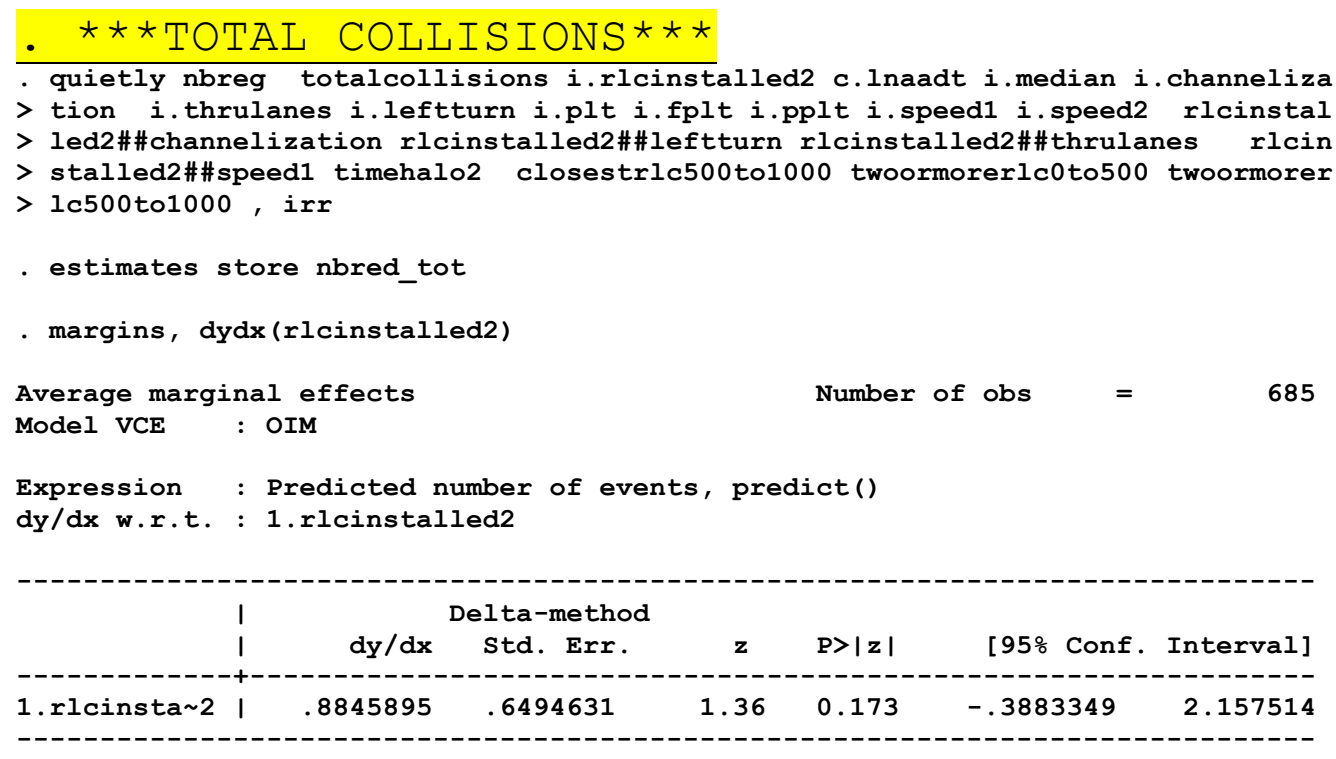

Note: $d y / d x$ for factor levels is the discrete change from the base level.

- quietly xtnbreg totalcollisions i.rlcinstalled2 c.lnaadt i.median i.channeli $>$ zation i.thrulanes i.leftturn i.plt i.fplt i.pplt i.speed2 rlcinstalled2\#\#me $>$ dian rlcinstalled2\#\#channelization rlcinstalled2\#\#leftturn rlcinstalled2\#\#thr 


\begin{tabular}{|c|c|c|c|c|c|c|}
\hline & 1 & Delta-method & & & & \\
\hline & $d y / d x$ & std. Err. & $\mathbf{z}$ & $\mathrm{P}>|\mathbf{z}|$ & [95\% Conf. & Interval] \\
\hline .rlcinsta 2 & I -.0288863 & .0432441 & -0.67 & 0.504 & -.1136433 & .0558707 \\
\hline
\end{tabular}

Note: dy/dx for factor levels is the discrete change from the base level.

\section{Table 4 - Total collisions - Best fit NB and XTNBREG regression models}

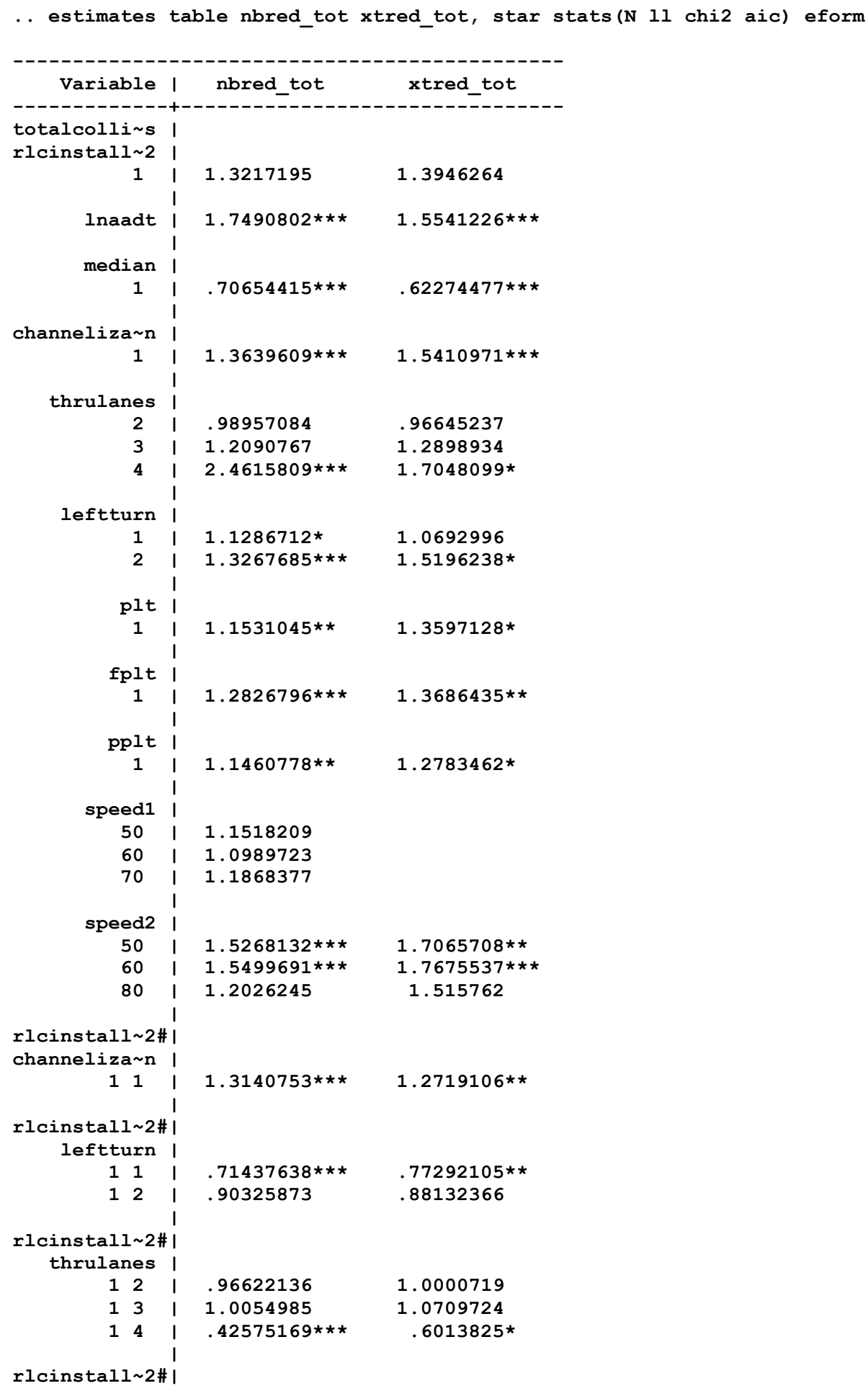




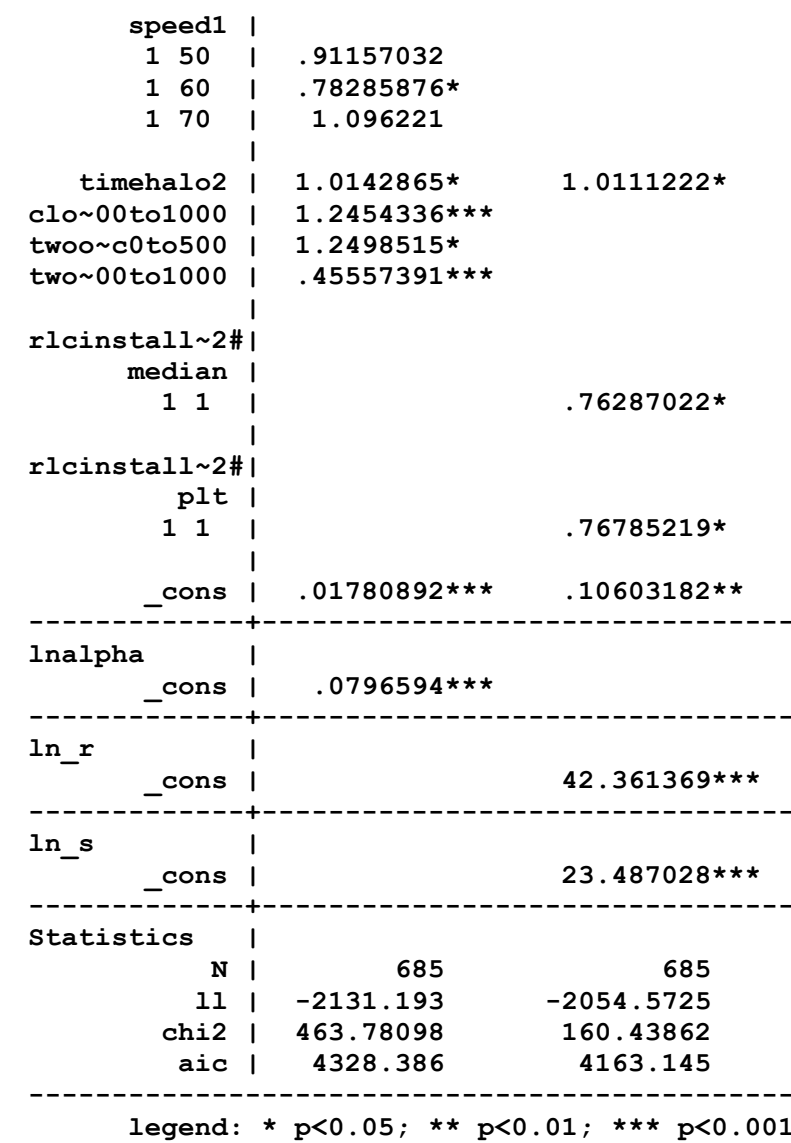

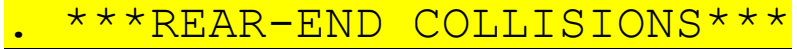

- quietly nbreg rearend i.rlcinstalled2 c.lnaadt i.median i.channelization i $>$.thrulanes i.leftturn i.plt i.fplt i.pplt i.speed1 i.speed2 rlcinstalled2\#\#ch $>$ annelization rlcinstalled2\#\#leftturn numcams timehalo2 closestrlc0to500 close $>$ strlc500to1000 twoormorerlc0to500 threewithinrlc1000, irr

- estimates store nbred_Rear

- margins, dydx(rlcinstalled2)
}

Average marginal effects

Model VCE

$$
\text { : OIM }
$$

Expression : Predicted number of events, predict()

$\mathrm{dy} / \mathrm{dx}$ w.r.t. : 1.rlcinstalled2

\begin{tabular}{|c|c|c|c|c|c|c|c|}
\hline & 1 & $d y / d x$ & $\begin{array}{l}\text { Delta-method } \\
\text { Std. Err. }\end{array}$ & $\mathbf{z}$ & $\mathrm{P}>|\mathbf{z}|$ & [95\% Conf. & Interval] \\
\hline 1.rlcinsta 2 & I & 635787 & .4528545 & 1.40 & 0.160 & -.2517915 & 1.52336 \\
\hline
\end{tabular}

Note: $d y / d x$ for factor levels is the discrete change from the base level.

quietly xtnbreg rearend i.rlcinstalled2 c.lnaadt i.median i.channelization $>$ i.thrulanes i.leftturn i.plt i.fplt i.pplt i.speed1 i.speed2 rlcinstalled2\#\#m $>$ edian rlcinstalled2\#\#channelization rlcinstalled2\#\#leftturn rlcinstalled2\#\#fp

$>$ it numcams timehalo2 closestrlc0to500 twoormorerlc0to500 threewithinrlc100

$>0$, irr re

- estimates store xtred_Rear 
- margins, dydx(rlcinstalled2)

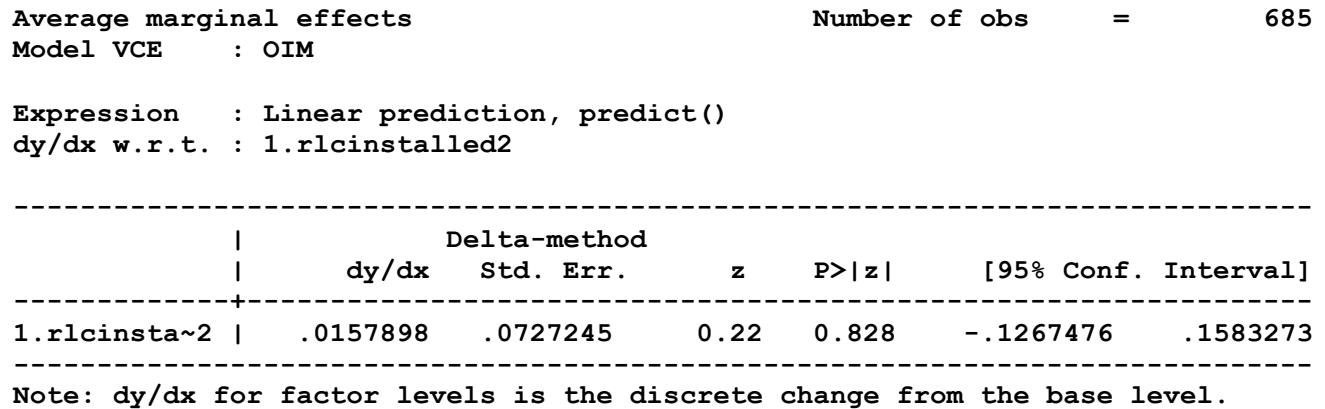

Table 5 - RE collisions - Best fit NB and XTNBREG regression models

- estimates table nbred_Rear xtred_Rear, star stats(N 11 chi2 aic) eform

\begin{tabular}{|c|c|c|c|}
\hline Variable & 1 & nbred_Rear & xtred_Rear \\
\hline & & & \\
\hline rearend & I & & \\
\hline rlcinstall 2 & i & & \\
\hline 1 & i & .89256089 & 1.1853131 \\
\hline & 1 & & \\
\hline Inaadt & 1 & $3.0504547 \star \star \star$ & $2.0208322 * \star \star$ \\
\hline & I & & \\
\hline median & 1 & & \\
\hline 1 & 1 & $1.735048 * \star \star$ & $1.8668862 * \star$ \\
\hline & I & & \\
\hline channeliza n & i & & \\
\hline 1 & 1 & 1.0303979 & 1.2650365 \\
\hline & I & & \\
\hline thrulanes & 1 & & \\
\hline 2 & i & $.46563974 * \star \star$ & $.4609894 * \star$ \\
\hline 3 & I & $.40045236 * \star \star *$ & $.41764803 * \star$ \\
\hline 4 & i & $.60151293 * \star *$ & .53501934 \\
\hline & I & & \\
\hline leftturn & I & & \\
\hline 1 & I & $.79740425 * \star$ & $.66061526 *$ \\
\hline 2 & i & $.68852284 * \star$ & .81849696 \\
\hline & I & & \\
\hline plt & i & & \\
\hline 1 & I & 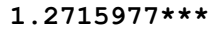 & 1. 4899393 * \\
\hline & i & & \\
\hline fplt & I & & \\
\hline 1 & i & $1.9535483 * \star \star$ & $2.2936995 * \star \star$ \\
\hline & I & & \\
\hline pplt & I & & \\
\hline 1 & i & $1.4745987 * \star \star$ & $1.8103629 * \star \star$ \\
\hline & i & & \\
\hline speed1 & 1 & & \\
\hline 50 & i & $1.6721987 * \star \star$ & 1.3996471 \\
\hline 60 & i & .91850205 & .71262692 \\
\hline 70 & i & $2.4424213 * \star \star *$ & $2.3533684 * \star \star$ \\
\hline & 1 & & \\
\hline speed2 & I & & \\
\hline 50 & I & $.52076907 * \star \star$ & $.57688957 *$ \\
\hline 60 & i & .94801827 & 1.2571618 \\
\hline 80 & i & $.10967925 * \star \star$ & $.06443402 * \star$ \\
\hline & 1 & & \\
\hline rlcinstall 2 & $\$ 1$ & & \\
\hline channeliza n & I & & \\
\hline 11 & i & $2.0445292 * \star \star$ & $1.5862499 * \star \star$ \\
\hline & I & & \\
\hline rlcinstall 2 & $\$ 1$ & & \\
\hline leftturn & 1 & & \\
\hline 11 & i & $.6812503 * \star$ & .69327182 * \\
\hline 12 & 1 & .71578329 & .68744844 * \\
\hline
\end{tabular}




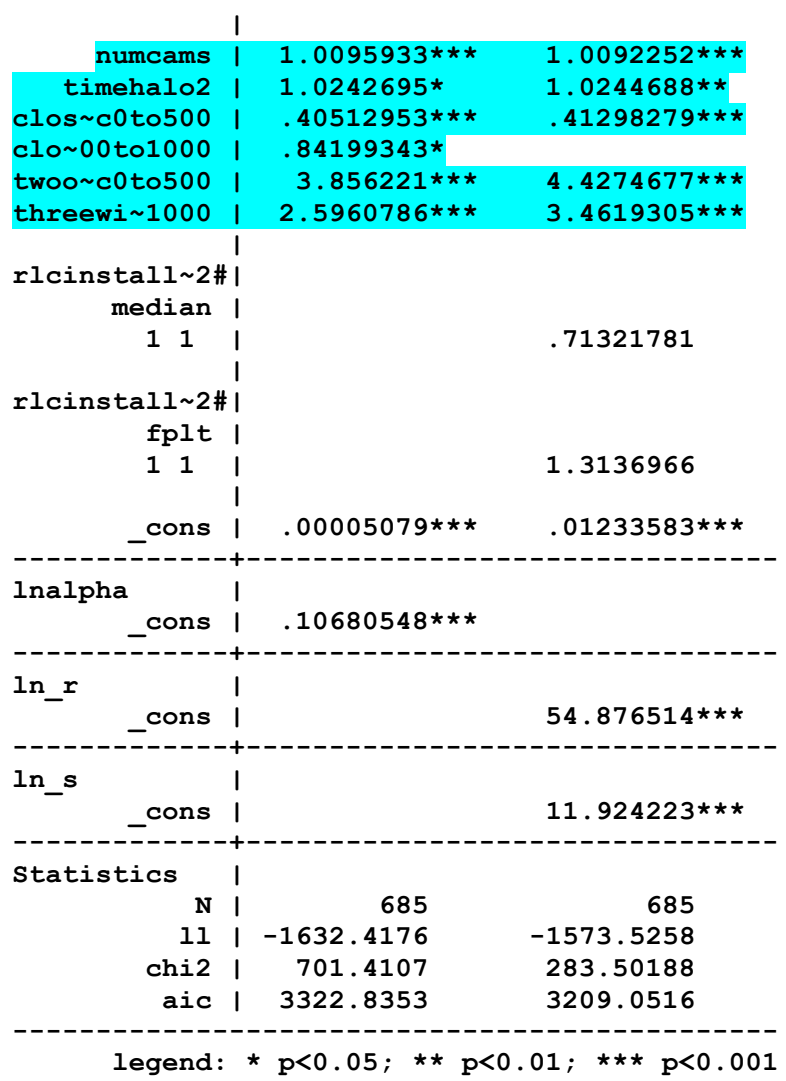

. $\quad * \star \star$ ANGLE COLLISIONS $* \star \star$

- quietly nbreg angle i.rlcinstalled2 c.lnaadt i.median i.channelization i.t $>$ hrulanes i.leftturn i.plt i.fplt i.pplt i.speedl rlcinstalled2\#\#median rlcins $>$ talled2\#\#channelization rlcinstalled2\#\#leftturn rlcinstalled2\#\#thrulanes rlci $>$ nstalled2\#\#fplt rlcinstalled2\#\#plt rlcinstalled2\#\#pplt numcams twoormorerlc5 $>00$ to1000, irr

- estimates store nbred_ANG

- margins, dydx(rlcinstalled2)

Average marginal effects $\quad$ Number of obs $=\quad 685$ Model VCE : OIM

Expression : Predicted number of events, predict() $\mathrm{dy} / \mathrm{dx}$ w.r.t. : 1.rlcinstalled2

\begin{tabular}{|c|c|c|c|c|c|c|}
\hline 1 & & elta-metho & & & & \\
\hline 1 & $d y / d x$ & Std. Err. & $\mathbf{z}$ & $P>|z|$ & [95\% Conf. & Interval] \\
\hline rlcinsta 2 & -.40668 & .2714681 & -1.50 & 0.134 & -.9387478 & .1253877 \\
\hline
\end{tabular}

Note: $d y / d x$ for factor levels is the discrete change from the base level.

quietly xtnbreg angle i.rlcinstalled2 c.lnaadt i.median i.channelization i. $>$ thrulanes i.leftturn i.plt i.pplt rlcinstalled2\#\#median rlcinstalled2\#\#plt $r$

$>$ lcinstalled2\#\#pplt numcams, irr re

- estimates store xtred_ANG

- margins, dydx(rlcinstalled2) 


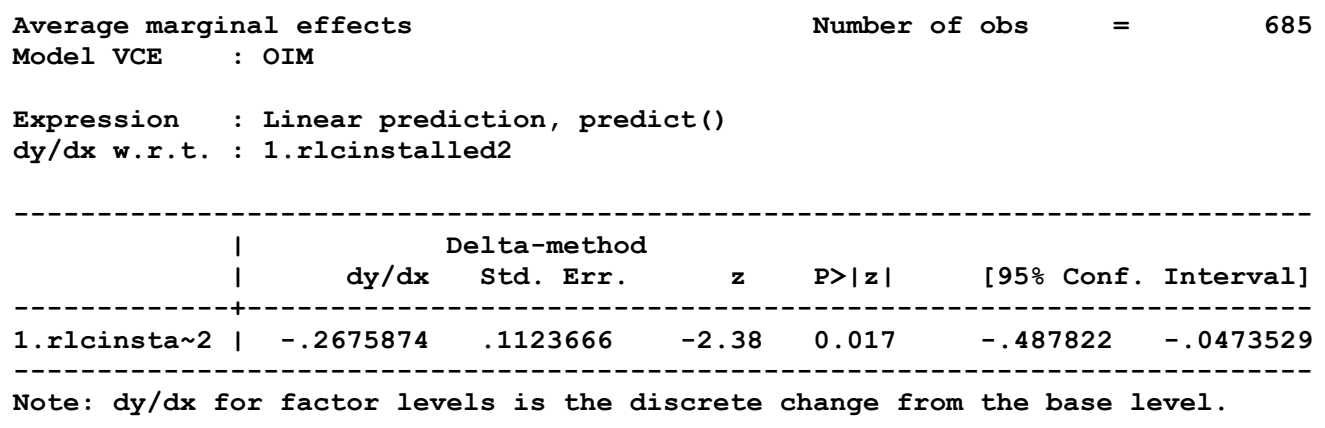

Table 6 - Angle collisions - Best fit NB and XTNBREG regression models

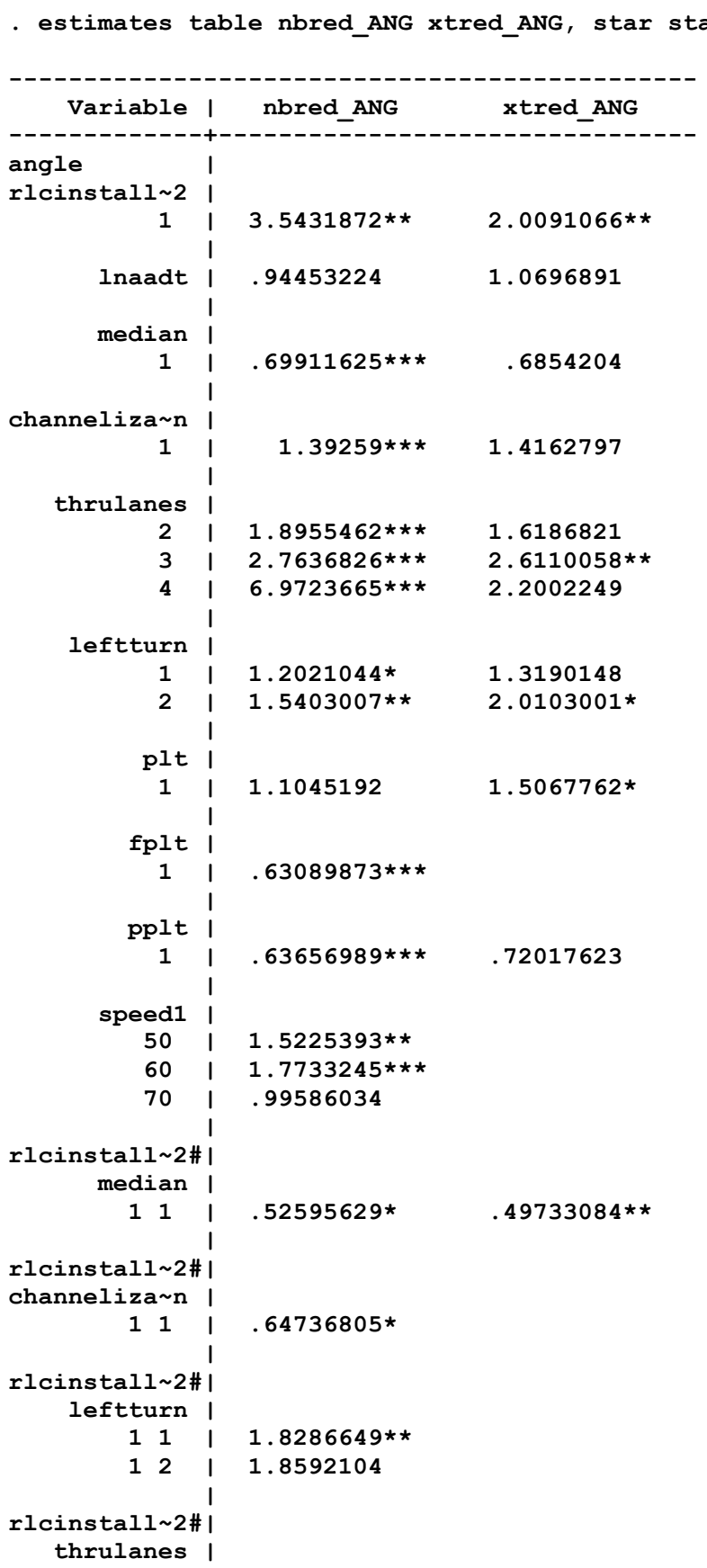




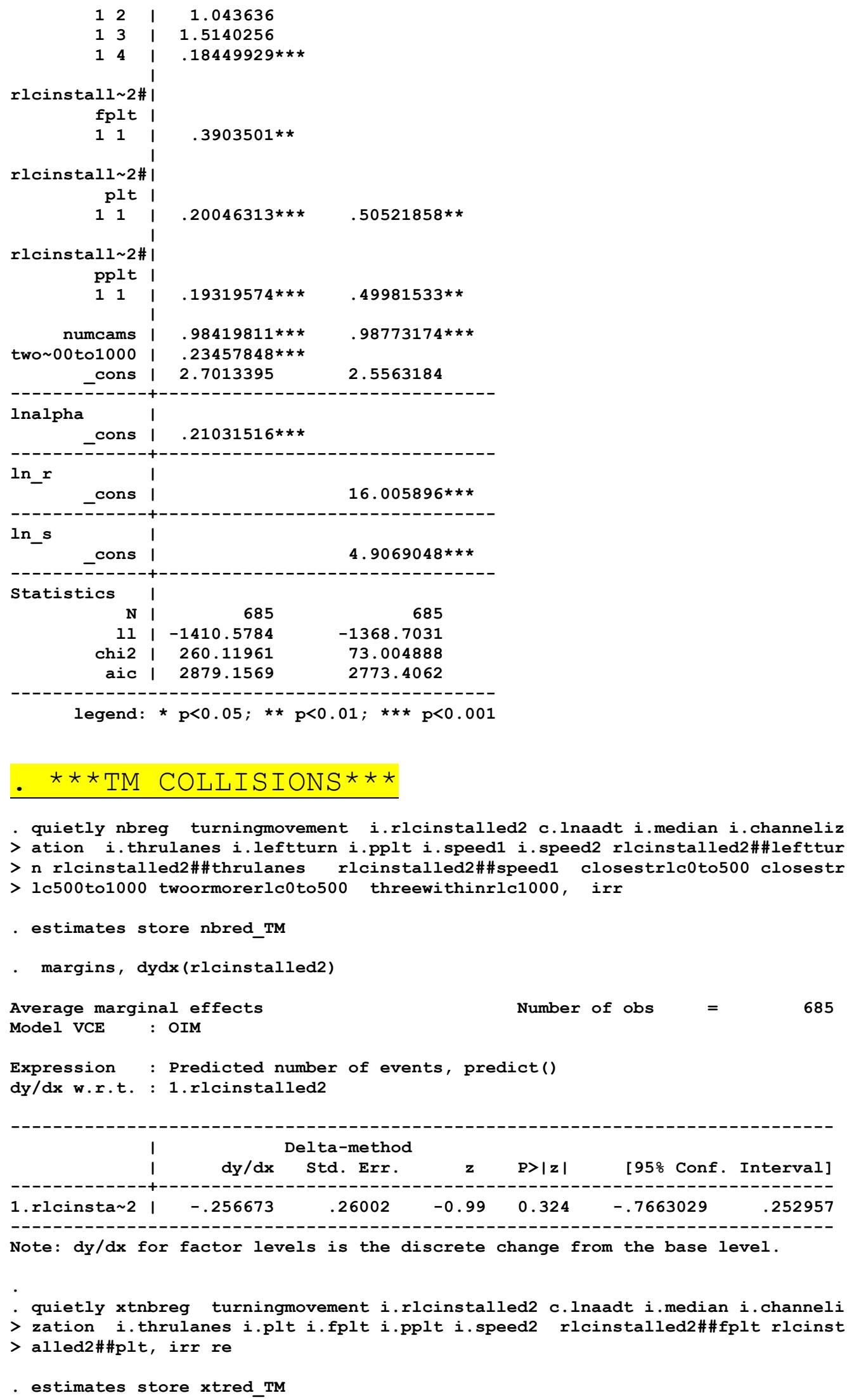


margins, dydx(rlcinstalled2)

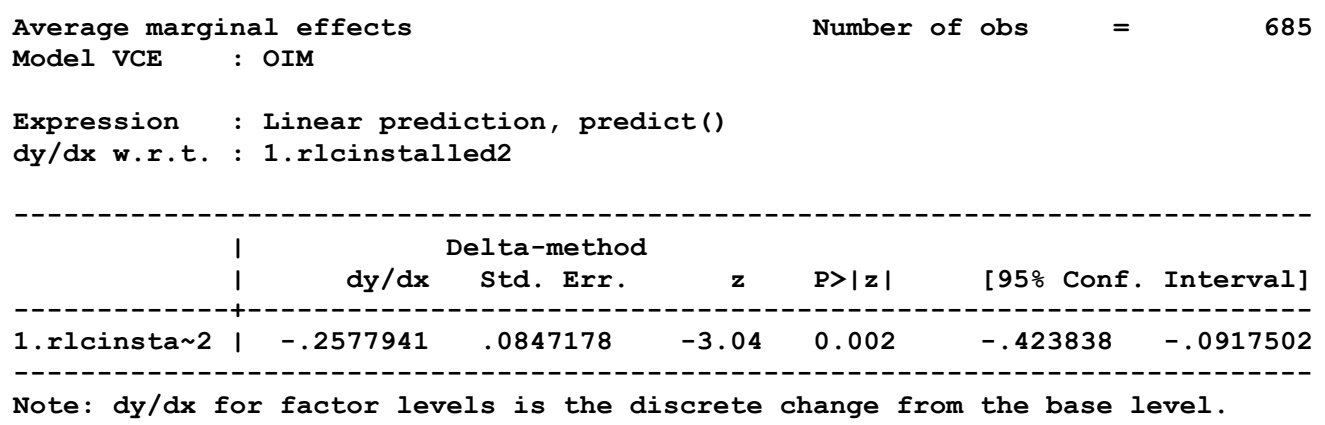

Table 7 - TM collisions - Best fit NB and XTNBREG regression models

- estimates table nbred_TM xtred_TM, star stats(N 11 chi2 aic) eform

\begin{tabular}{|c|c|c|c|}
\hline Variable & 1 & nbred_TM & xtred_TM \\
\hline \multicolumn{4}{|c|}{ turningmov $t$} \\
\hline \multicolumn{4}{|c|}{ rlcinstall 2 | } \\
\hline 1 & 1 & 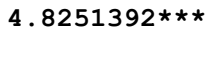 & 1.1665452 \\
\hline \multirow[t]{2}{*}{ lnaadt } & i & $1.5684963 * \star \star$ & 1.3519232 * \\
\hline & 1 & & \\
\hline \multicolumn{4}{|c|}{ median I } \\
\hline 1 & 1 & $.32223078 * \star \star *$ & $.51569926 * \star$ \\
\hline \multirow{2}{*}{\multicolumn{4}{|c|}{ channeliza n | }} \\
\hline & & & \\
\hline 1 & $\begin{array}{l}1 \\
1\end{array}$ & 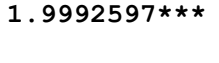 & 1.4020666 \\
\hline \multicolumn{4}{|c|}{ thrulanes } \\
\hline 2 & 1 & 1.2160026 & .57589228 \\
\hline 3 & I & 1.1655562 & .73928375 \\
\hline 4 & 1 & $2.2385955 * \star \star$ & 1.2555515 \\
\hline & I & & \\
\hline \multicolumn{4}{|c|}{ leftturn | } \\
\hline 1 & 1 & 1.0634158 & \\
\hline 2 & 1 & 1.0595387 & \\
\hline & 1 & & \\
\hline \multicolumn{4}{|c|}{ pplt I } \\
\hline 1 & 1 & $1.6049313 * \star \star$ & 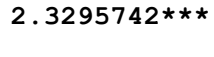 \\
\hline \multicolumn{4}{|c|}{ speed1 } \\
\hline $\begin{array}{c}\text { speed1 } \\
50\end{array}$ & 1 & $.58861143 * \star$ & \\
\hline 60 & 1 & .83790931 & \\
\hline \multirow[t]{2}{*}{70} & 1 & $.42829683 * \star \star *$ & \\
\hline & 1 & & \\
\hline \multicolumn{4}{|c|}{ speed2 I } \\
\hline 50 & 1 & 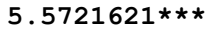 & $3.3178915 * \star \star$ \\
\hline 60 & I & 3. $3628425 * \star \star$ & $2.3845262 \star$ \\
\hline \multirow[t]{2}{*}{80} & I & $15.521402 \star * \star$ & 3.2914878 \\
\hline \multirow{2}{*}{\multicolumn{4}{|c|}{ rlcinstall 2\# }} \\
\hline & & & \\
\hline \multicolumn{4}{|c|}{ leftturn 1} \\
\hline 11 & 1 & 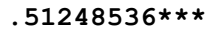 & \\
\hline \multirow[t]{2}{*}{12} & 1 & 1.1300569 & \\
\hline & 1 & & \\
\hline \multicolumn{4}{|c|}{ rlcinstall 2\#। } \\
\hline \multicolumn{4}{|c|}{ thrulanes | } \\
\hline 12 & 1 & $.4962003^{*}$ & \\
\hline 13 & 1 & .55358242 & \\
\hline \multirow[t]{2}{*}{14} & 1 & $.18446331 \star \star \star *$ & \\
\hline & 1 & & \\
\hline \multicolumn{4}{|c|}{ rlcinstall 2\#| } \\
\hline speed1 & 1 & & \\
\hline 150 & I & 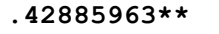 & \\
\hline
\end{tabular}




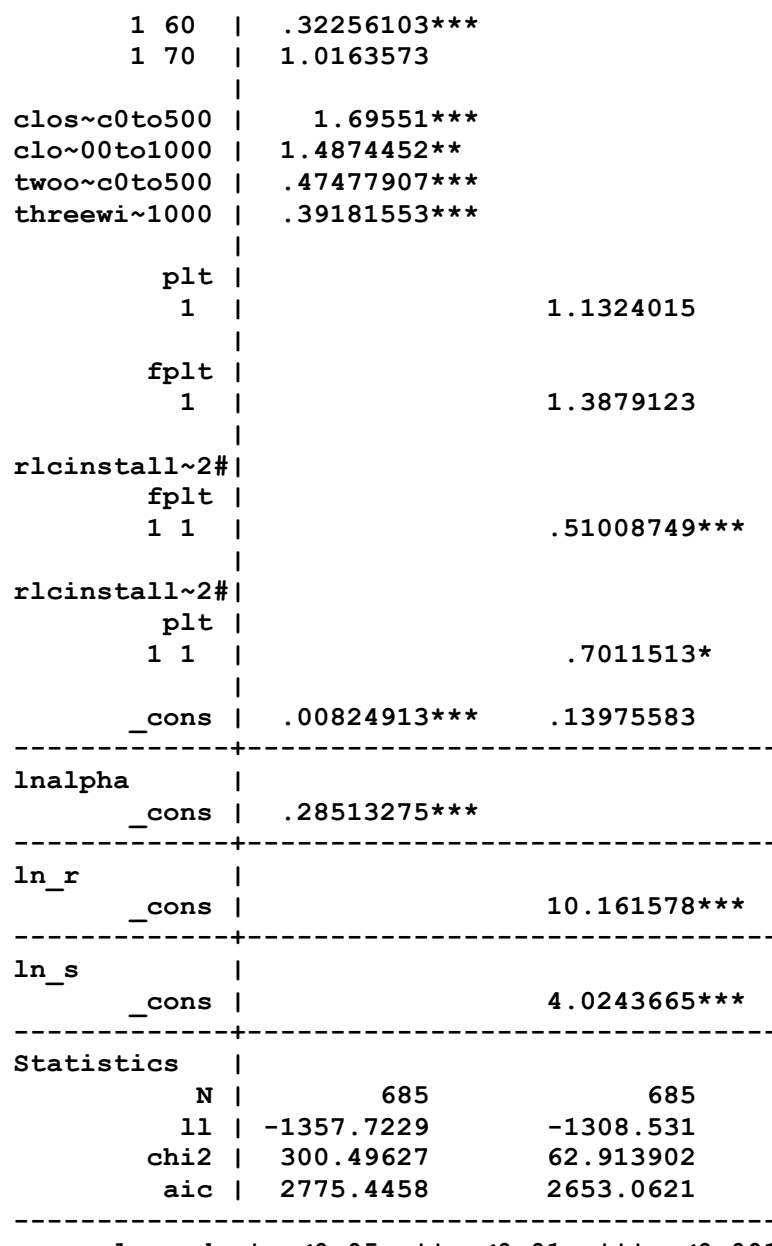

legend: * $\mathrm{p}<0.05 ; \star \star \mathrm{p}<0.01 ; \star \star \star \mathrm{p}<0.001$

. $\quad \star \star \star$ Sideswipe COLLISIONS * * $\star$

- quietly nbreg sideswipe i.rlcinstalled2 c.lnaadt i.median i.channelization

$>$ i.thrulanes $i$.leftturn i.plt i.fplt i.pplt $i$.speed1 i.speed2 rlcinstalled2\#\#

$>$ median rlcinstalled2\#\#channelization rlcinstalled2\#\#leftturn rlcinstalled2\#\#t

$>$ hrulanes rlcinstalled2\#\#fplt numcams timehalo closestrlc500to1000 twoorm

$>$ orerlc500to1000 threewithinrlc1000, irr

- estimates store nbred_ss

- margins, dydx(rlcinstalled2)

Average marginal effects

Number of obs =

685

Model VCE : OIM

Expression : Predicted number of events, predict()

dy/dx w.r.t. : 1.rlcinstalled2

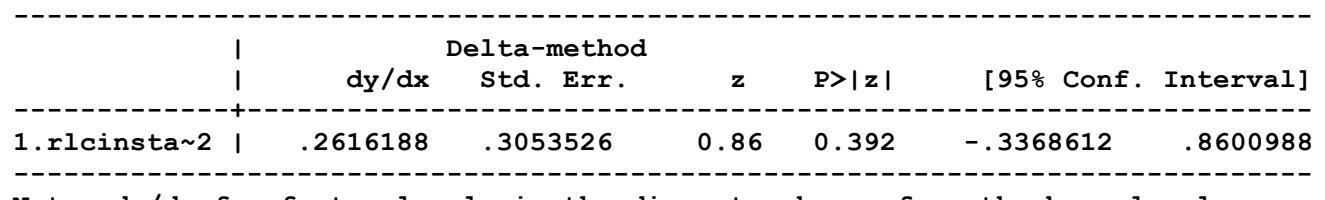

Note: $\mathrm{dy} / \mathrm{dx}$ for factor levels is the discrete change from the base level.

- quietly xtnbreg sideswipe i.rlcinstalled2 c.lnaadt i.median i.channelization i.thrulanes i.leftturn i.plt i.fplt i.speed2 rlcinstalled2\#\#median rlcin 


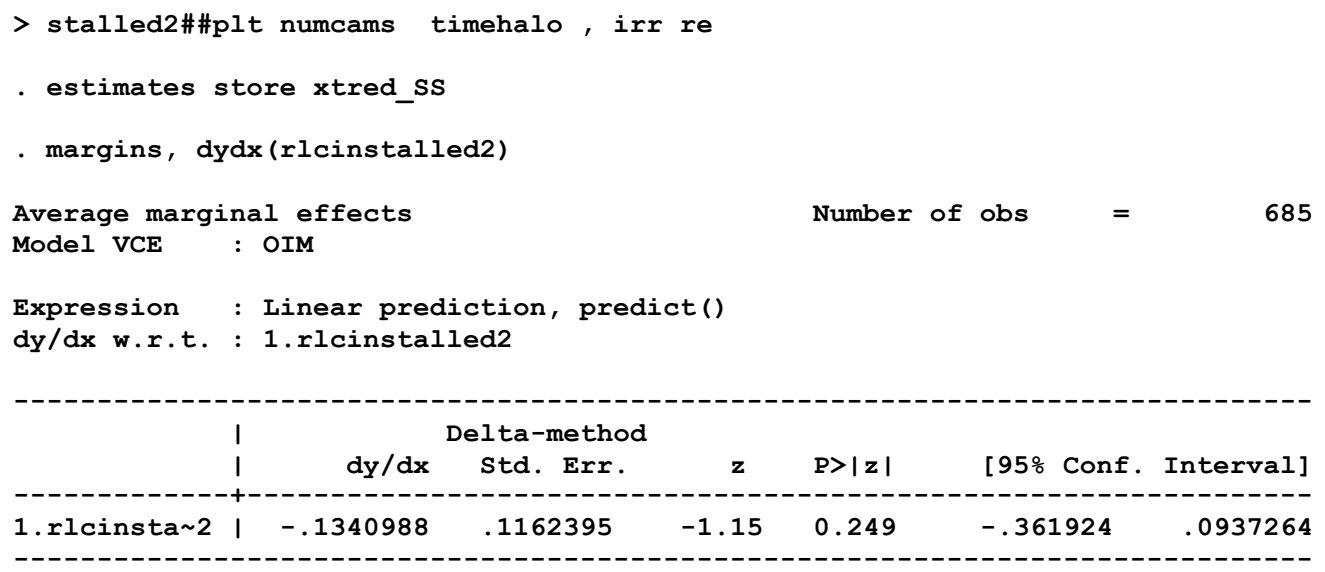

Note: dy/dx for factor levels is the discrete change from the base level.

Table 8 - SS collisions - Best fit NB and XTNBREG regression models - estimates table nbred_SS xtred_SS, star stats(N 11 chi2 aic) eform

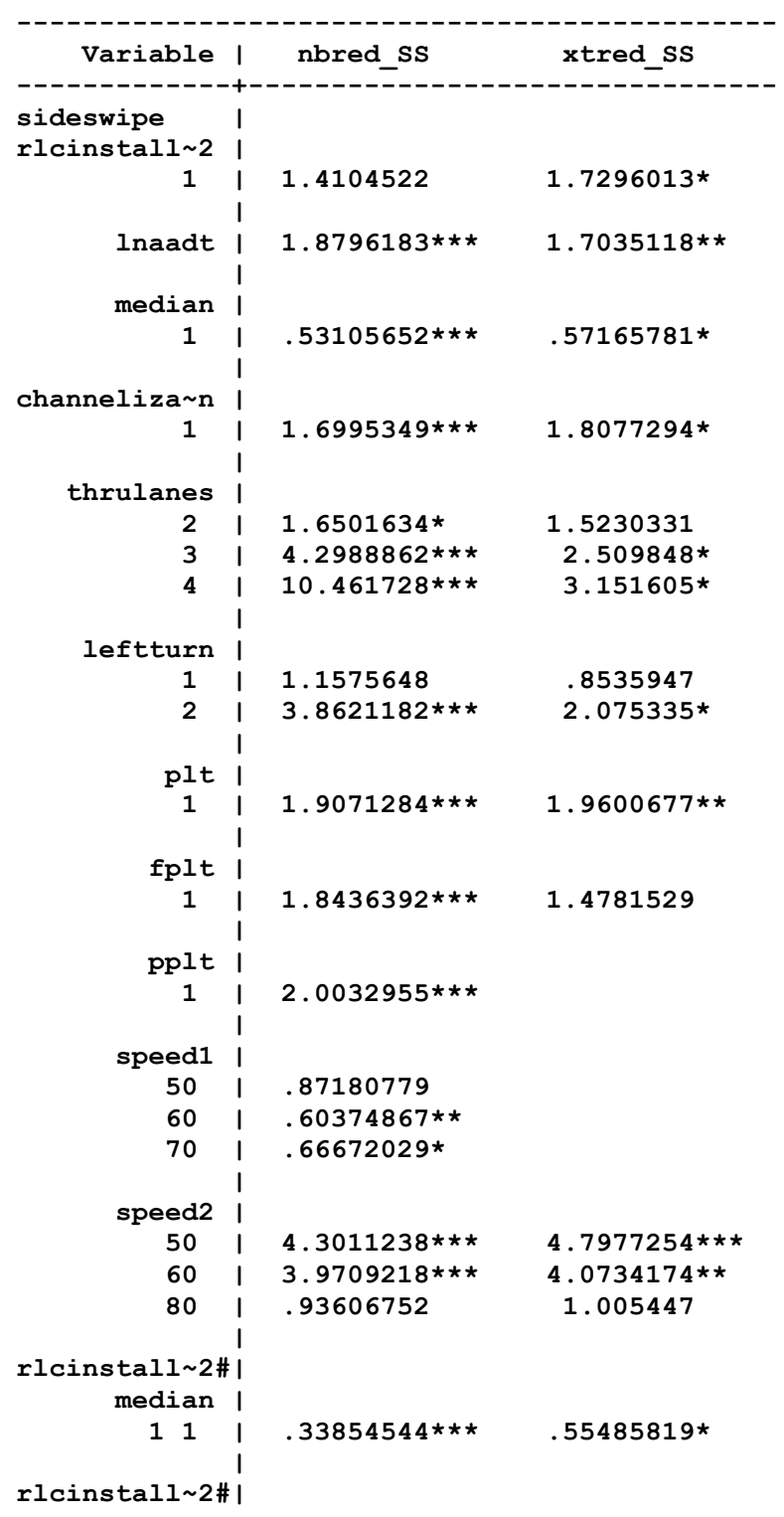




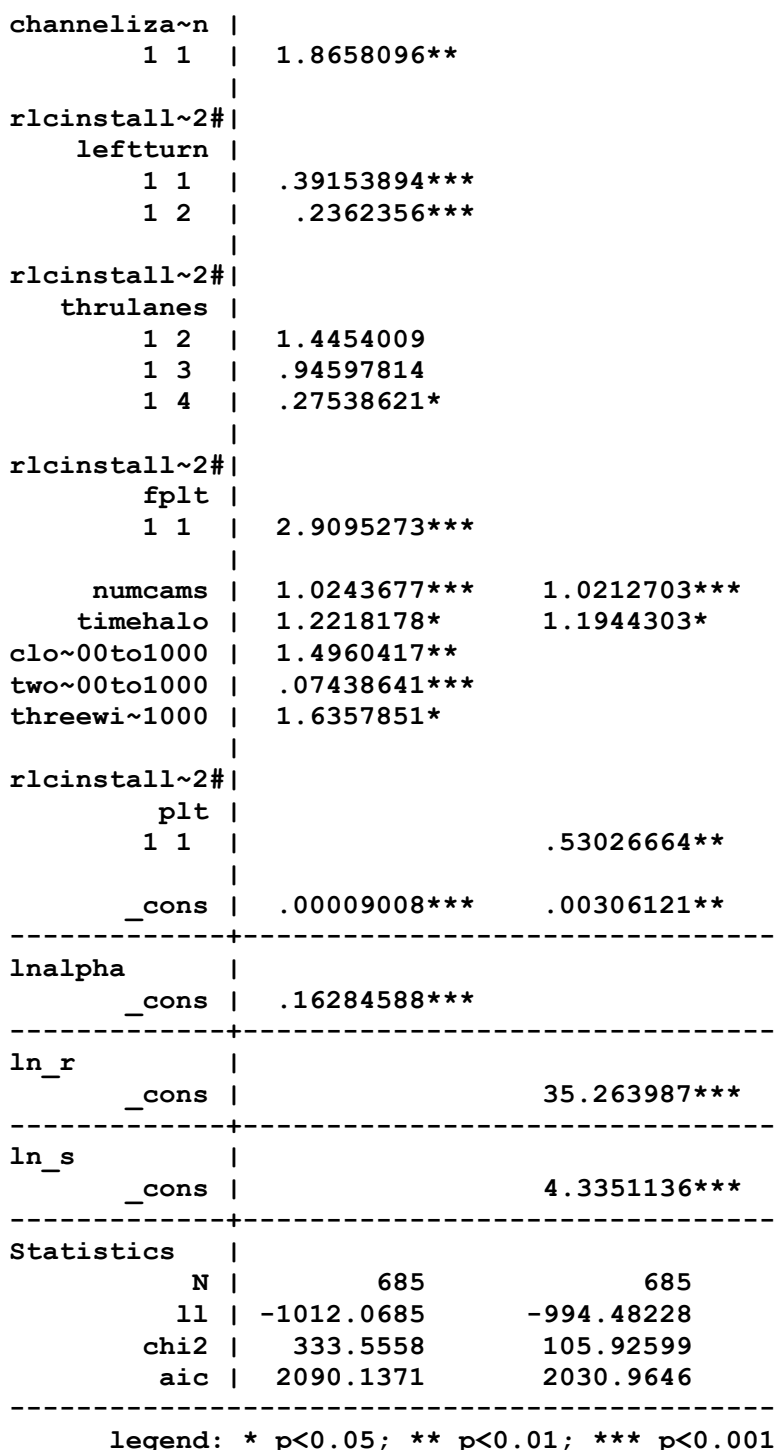




\section{Appendix J - EB Models}

How variables were calculated in the following tables:

[1] Average observed Total collisions/yr BEFORE Cam: count

[2] Average observed Total collisions/yr After Cam: count

[3] Predicted w/o cam using SPF Model $=$ cons_coeff*AADT ${ }^{\wedge}$ coeff

[4] weight $=1 /\left(1+\left([3]^{*}\right.\right.$ years $) /$ overdispersion parameter $)$

[5] Expected Collisions (EB estimate) $=[4] *[3]+(1-[4]) *[1]$

[6] Observed \% change (before after $=([2]-[1]) /[1]$

[7] Observed $\%$ change (before after) $)=([2]-[5]) /[5]$ 
SPF Models calculated using nbreg from 14 non-RLC intersections

TOTAL COLLISIONS

\begin{tabular}{|c|c|c|c|c|c|c|c|c|c|}
\hline & Intersection & AADT & $\begin{array}{l}\text { Average } \\
\text { observed } \\
\text { Total } \\
\text { collisions/yr } \\
\text { BEFORE } \\
\text { Cam } \\
\end{array}$ & $\begin{array}{l}\text { Average } \\
\text { observed } \\
\text { Total } \\
\text { collisions/yr } \\
\text { After Cam } \\
\end{array}$ & $\begin{array}{l}\text { Predicted } \\
\text { w/o cam } \\
\text { using SPF } \\
\text { Model } \\
\end{array}$ & weight & $\begin{array}{l}\text { Expected } \\
\text { Collisions } \\
\text { (EB } \\
\text { estimate) } \\
\end{array}$ & $\begin{array}{l}\text { Observed } \\
\% \text { change } \\
\text { (before } \\
\text { after) }\end{array}$ & $\begin{array}{l}\text { \% change } \\
\text { (expected } \\
\& \\
\text { observed } \\
\text { after) } \\
\end{array}$ \\
\hline 1 & BRONSON AVE \& CARLING AVE & 43,684 & 18.0 & 15.2 & 0.5623 & 0.02 & 17.6 & $-16 \%$ & $-14 \%$ \\
\hline 2 & CARLING AVE \& RICHMOND RD & 46,299 & 11.0 & 14.1 & 0.5623 & 0.02 & 10.8 & $28 \%$ & $31 \%$ \\
\hline 3 & COVENTRY RD \& ST. LAURENT BLVD & 52,562 & 21.0 & 24.3 & 0.5623 & 0.02 & 20.6 & $15 \%$ & $18 \%$ \\
\hline 4 & EAGLESON RD \& HAZELDEAN RD & 37,775 & 18.5 & 16.2 & 0.5623 & 0.02 & 18.1 & $-13 \%$ & $-11 \%$ \\
\hline 5 & HERON RD \& RIVERSIDE DR & 58,882 & 27.5 & 29.3 & 0.5623 & 0.02 & 26.9 & $7 \%$ & $9 \%$ \\
\hline 6 & MEADOWLANDS DR \& MERIVALE RD & 47,966 & 20.0 & 23.0 & 0.5623 & 0.02 & 19.6 & $15 \%$ & $18 \%$ \\
\hline 7 & ALBERT ST \& KENT ST (OTTAWA) & 50,000 & 11.5 & 12.0 & 0.5623 & 0.02 & 11.3 & $4 \%$ & $7 \%$ \\
\hline 8 & BOOTH ST \& OTTAWA RIVER PKWY & 46,168 & 9.8 & 10.4 & 0.5623 & 0.02 & 9.6 & $6 \%$ & $9 \%$ \\
\hline 9 & BASELINE RD \& MERIVALE RD & 46,821 & 26.2 & 16.2 & 0.5623 & 0.02 & 25.7 & $-38 \%$ & $-37 \%$ \\
\hline 10 & CARLING AVE \& ISLAND PARK DR & 23,533 & 10.2 & 8.8 & 0.5623 & 0.02 & 10.0 & $-14 \%$ & $-12 \%$ \\
\hline 11 & CAMPEAU DR \& MARCH RD & 59,992 & 20.4 & 18.6 & 0.5623 & 0.02 & 20.0 & $-9 \%$ & $-7 \%$ \\
\hline 12 & BANK ST \& RIVERSIDE DR N & 41,598 & 16.2 & 15.8 & 0.5623 & 0.02 & 15.9 & $-3 \%$ & $0 \%$ \\
\hline 13 & BELFAST RD \& ST. LAURENT BLVD & 38,197 & 21.9 & 28.0 & 0.5623 & 0.02 & 21.4 & $28 \%$ & $31 \%$ \\
\hline 14 & CHARLEMAGNE BLVD N \& TENTH LINE RD & 35,827 & 14.6 & 20.0 & 0.5623 & 0.02 & 14.2 & $37 \%$ & $40 \%$ \\
\hline 15 & BANK ST \& HUNT CLUB RD & 50,684 & 19.7 & 25.6 & 0.5623 & 0.02 & 19.2 & $30 \%$ & $33 \%$ \\
\hline 16 & CATHERINE ST \& HWY 417 O'CONN & 50,000 & 17.8 & 12.0 & 0.5623 & 0.02 & 17.4 & $-33 \%$ & $-31 \%$ \\
\hline 17 & CATHERINE ST \& KENT ST & 50,000 & 20.9 & 23.0 & 0.5623 & 0.02 & 20.4 & $10 \%$ & $13 \%$ \\
\hline 18 & RUSSELL RD \& WALKLEY RD & 34,805 & 21.9 & 30.4 & 0.5623 & 0.02 & 21.4 & $39 \%$ & $42 \%$ \\
\hline 19 & BRONSON AVE \& COMMISSIONER ST & 18,746 & 14.0 & 8.8 & 0.5623 & 0.02 & 13.7 & $-38 \%$ & $-36 \%$ \\
\hline 20 & FISHER AVE \& MEADOWLANDS DR & 23,176 & 13.3 & 12.5 & 0.5623 & 0.02 & 13.0 & $-6 \%$ & $-4 \%$ \\
\hline 21 & DUFORD DR \& ST. JOSEPH BLVD & 25,612 & 8.5 & 7.0 & 0.5623 & 0.02 & 8.3 & $-17 \%$ & $-15 \%$ \\
\hline 22 & AVIATION PKWY \& OGILVIE RD & 40,664 & 16.6 & 13.8 & 0.5623 & 0.02 & 16.3 & $-17 \%$ & $-16 \%$ \\
\hline
\end{tabular}




\begin{tabular}{|c|c|c|c|c|c|c|c|c|c|}
\hline 23 & BANK ST \& HERON RD & 56,511 & 22.5 & 22.5 & 0.5623 & 0.02 & 22.0 & $\mathbf{0} \%$ & $2 \%$ \\
\hline 24 & BERRIGAN DR \& GREENBANK RD & 25,292 & 9.7 & 11.3 & 0.5623 & 0.02 & 9.5 & $16 \%$ & $18 \%$ \\
\hline 25 & CONROY RD \& LORRY GREENBERG DR & 22,615 & 3.0 & 2.5 & 0.5623 & 0.02 & 2.9 & $-17 \%$ & $-15 \%$ \\
\hline 26 & JOCKVALE RD \& STRANDHERD DR & 17,536 & 3.8 & 5.5 & 0.5623 & 0.02 & 3.7 & $44 \%$ & $47 \%$ \\
\hline 27 & CYRVILLE RD \& INNES RD & 55,925 & 15.2 & 14.5 & 0.5623 & 0.02 & 14.9 & $-4 \%$ & $-2 \%$ \\
\hline 28 & $\begin{array}{l}\text { HAWTHORNE AVE \& QUEEN ELIZABETH } \\
\text { DRWY }\end{array}$ & 27,065 & 8.5 & 9.0 & 0.5623 & 0.02 & 8.3 & $6 \%$ & $9 \%$ \\
\hline 29 & ALBERT ST \& BOOTH ST & 27,558 & 12.9 & 12.8 & 0.5623 & 0.02 & 12.6 & $-1 \%$ & $1 \%$ \\
\hline 30 & DRIVEWAY (THE) \& ISLAND PARK DR & 19,583 & 8.5 & 8.5 & 0.5623 & 0.02 & 8.4 & $-1 \%$ & $2 \%$ \\
\hline 31 & BLAIR RD \& BLAIR RD RAMP 36 & 30,969 & 16.9 & 25.3 & 0.5623 & 0.02 & 16.5 & $49 \%$ & $53 \%$ \\
\hline 32 & KING EDWARD AVE \& ST. PATRICK ST & 33,216 & 25.5 & 9.0 & 0.5623 & 0.02 & 25.0 & $-65 \%$ & $-64 \%$ \\
\hline 33 & SAUNDERSON DR \& SMYTH RD & 49,546 & 4.2 & 5.5 & 0.5623 & 0.02 & 4.1 & $32 \%$ & $35 \%$ \\
\hline 34 & KING EDWARD AVE \& ST. ANDREW ST & 47,702 & 4.0 & 1.5 & 0.5623 & 0.02 & 3.9 & $-63 \%$ & $-62 \%$ \\
\hline & Total & & 514 & 513 & & & 503 & $0 \%$ & $1.82 \%$ \\
\hline
\end{tabular}

\section{REAR END COLLISIONS}

\begin{tabular}{|c|c|c|c|c|c|c|c|c|c|}
\hline & Intersection & AADT & $\begin{array}{l}\text { Average } \\
\text { observed } \\
\text { Rear end } \\
\text { collisions/yr } \\
\text { BEFORE } \\
\text { Cam }\end{array}$ & $\begin{array}{l}\text { Average } \\
\text { observed } \\
\text { Rearend } \\
\text { coll/yr }\end{array}$ & $\begin{array}{l}\text { Predicted } \\
\text { w/o cam } \\
\text { using SPF } \\
\text { Model }\end{array}$ & weight & $\begin{array}{l}\text { Expected } \\
\text { w/o cam } \\
\text { (EB } \\
\text { estimate) }\end{array}$ & $\begin{array}{l}\text { Observed } \\
\% \text { change }\end{array}$ & $\begin{array}{l}\text { \% change } \\
\text { (expected } \\
\& \\
\text { observed } \\
\text { after) }\end{array}$ \\
\hline 1 & BRONSON AVE \& CARLING AVE & 43,684 & 5.0 & 4.7 & -1.0674 & -0.01 & 5.1 & $-6 \%$ & $-7 \%$ \\
\hline 2 & CARLING AVE \& RICHMOND RD & 46,299 & 6.0 & 7.5 & -1.0674 & -0.01 & 6.1 & $24 \%$ & $23 \%$ \\
\hline 3 & COVENTRY RD \& ST. LAURENT BLVD & 52,562 & 7.5 & 12.7 & -1.0674 & -0.01 & 7.6 & $69 \%$ & $67 \%$ \\
\hline 4 & EAGLESON RD \& HAZELDEAN RD & 37,775 & 9.5 & 10.9 & -1.0674 & -0.01 & 9.6 & $15 \%$ & $14 \%$ \\
\hline 5 & HERON RD \& RIVERSIDE DR & 58,882 & 17.5 & 22.1 & -1.0674 & -0.01 & 17.7 & $26 \%$ & $25 \%$ \\
\hline 6 & MEADOWLANDS DR \& MERIVALE RD & 47,966 & 10.5 & 9.8 & -1.0674 & -0.01 & 10.6 & $-6 \%$ & $-7 \%$ \\
\hline 7 & ALBERT ST \& KENT ST (OTTAWA) & 50,000 & 1.0 & 0.3 & -1.0674 & -0.01 & 1.0 & $-67 \%$ & $-67 \%$ \\
\hline 8 & BANK ST \& HUNT CLUB RD & 50,684 & 2.8 & 7.7 & -1.0674 & -0.01 & 2.8 & $177 \%$ & $173 \%$ \\
\hline 9 & BANK ST \& RIVERSIDE DR N & 41,598 & 12.9 & 8.5 & -1.0674 & -0.01 & 13.0 & $-34 \%$ & $-35 \%$ \\
\hline
\end{tabular}




\begin{tabular}{|c|c|c|c|c|c|c|c|c|c|}
\hline 10 & BASELINE RD \& MERIVALE RD & 46,821 & 1.1 & 6.3 & -1.0674 & -0.01 & 1.1 & $467 \%$ & $456 \%$ \\
\hline 11 & BELFAST RD \& ST. LAURENT BLVD & 38,197 & 12.9 & 6.4 & -1.0674 & -0.01 & 13.0 & $-50 \%$ & $-51 \%$ \\
\hline 12 & BOOTH ST \& OTTAWA RIVER PKWY & 46,168 & 4.1 & 10.1 & -1.0674 & -0.01 & 4.2 & $146 \%$ & $142 \%$ \\
\hline 13 & CAMPEAU DR \& MARCH RD & 59,992 & 5.6 & 10.6 & -1.0674 & -0.01 & 5.6 & $91 \%$ & $88 \%$ \\
\hline 14 & CARLING AVE \& ISLAND PARK DR & 23,533 & 6.2 & 7.2 & -1.0673 & -0.01 & 6.3 & $16 \%$ & $14 \%$ \\
\hline 15 & CHARLEMAGNE BLVD N \& TENTH LINE RD & 35,827 & 10.3 & 12.7 & -1.0674 & -0.01 & 10.5 & $23 \%$ & $22 \%$ \\
\hline 16 & CATHERINE ST \& HWY 417 O'CONN & 50,000 & 2.7 & 4.1 & -1.0674 & -0.01 & 2.7 & $54 \%$ & $52 \%$ \\
\hline 17 & CATHERINE ST \& KENT ST & 50,000 & 0.8 & 1.6 & -1.0674 & -0.01 & 0.8 & $106 \%$ & $101 \%$ \\
\hline 18 & RUSSELL RD \& WALKLEY RD & 34,805 & 12.6 & 15.1 & -1.0673 & -0.01 & 12.7 & $20 \%$ & $19 \%$ \\
\hline 19 & ALBERT ST \& BOOTH ST & 27,558 & 1.5 & 1.0 & -1.0673 & -0.01 & 1.6 & $-35 \%$ & $-36 \%$ \\
\hline 20 & AVIATION PKWY \& OGILVIE RD & 40,664 & 2.3 & 1.8 & -1.0674 & -0.01 & 2.3 & $-23 \%$ & $-24 \%$ \\
\hline 21 & BANK ST \& HERON RD & 56,511 & 2.9 & 2.3 & -1.0674 & -0.01 & 3.0 & $-23 \%$ & $-24 \%$ \\
\hline 22 & BERRIGAN DR \& GREENBANK RD & 25,292 & 4.7 & 6.8 & -1.0673 & -0.01 & 4.8 & $43 \%$ & $41 \%$ \\
\hline 23 & BLAIR RD \& BLAIR RD RAMP 36 & 30,969 & 8.5 & 11.3 & -1.0673 & -0.01 & 8.6 & $32 \%$ & $30 \%$ \\
\hline 24 & BRONSON AVE \& COMMISSIONER ST & 18,746 & 6.1 & 7.0 & -1.0673 & -0.01 & 6.2 & $15 \%$ & $14 \%$ \\
\hline 25 & CONROY RD \& LORRY GREENBERG DR & 22,615 & 0.5 & 1.3 & -1.0673 & -0.01 & 0.6 & $129 \%$ & $122 \%$ \\
\hline 26 & CYRVILLE RD \& INNES RD & 55,925 & 0.6 & 2.3 & -1.0674 & -0.01 & 0.7 & $254 \%$ & $244 \%$ \\
\hline 27 & DRIVEWAY (THE) \& ISLAND PARK DR & 19,583 & 8.0 & 9.3 & -1.0673 & -0.01 & 8.1 & $16 \%$ & $14 \%$ \\
\hline 28 & DUFORD DR \& ST. JOSEPH BLVD & 25,612 & 2.2 & 3.3 & -1.0673 & -0.01 & 2.2 & $49 \%$ & $47 \%$ \\
\hline 29 & FISHER AVE \& MEADOWLANDS DR & 23,176 & 3.5 & 3.3 & -1.0673 & -0.01 & 3.6 & $-8 \%$ & $-10 \%$ \\
\hline 30 & $\begin{array}{l}\text { HAWTHORNE AVE \& QUEEN ELIZABETH } \\
\text { DRWY }\end{array}$ & 27,065 & 0.7 & 1.5 & -1.0673 & -0.01 & 0.7 & $106 \%$ & $101 \%$ \\
\hline 31 & JOCKVALE RD \& STRANDHERD DR & 17,536 & 10.4 & 14.0 & -1.0673 & -0.01 & 10.5 & $35 \%$ & $34 \%$ \\
\hline 32 & KING EDWARD AVE \& ST. PATRICK ST & 33,216 & 6.9 & 3.0 & -1.0673 & -0.01 & 7.0 & $-57 \%$ & $-57 \%$ \\
\hline 33 & SAUNDERSON DR \& SMYTH RD & 49,546 & 0.8 & 3.0 & -1.0674 & -0.01 & 0.8 & $300 \%$ & $290 \%$ \\
\hline \multirow[t]{2}{*}{34} & KING EDWARD AVE \& ST. ANDREW ST & 47,702 & 2.9 & 1.0 & -1.0674 & -0.01 & 3.0 & $-66 \%$ & $-66 \%$ \\
\hline & Total & & 192 & 230 & & & 194 & $20 \%$ & $15.69 \%$ \\
\hline
\end{tabular}


ANGLE COLLISIONS

\begin{tabular}{|c|c|c|c|c|c|c|c|c|c|}
\hline & Intersection & $\mathrm{AADT}$ & $\begin{array}{l}\text { Average } \\
\text { observed } \\
\text { Angle } \\
\text { collisions/yr } \\
\text { BEFORE } \\
\text { Cam } \\
\end{array}$ & $\begin{array}{l}\text { Average } \\
\text { observed } \\
\text { Angle } \\
\text { AFTER cam } \\
\text { coll/yr } \\
\end{array}$ & $\begin{array}{l}\text { Predicted } \\
\text { w/o cam } \\
\text { using SPF } \\
\text { Model } \\
\end{array}$ & weight & $\begin{array}{l}\text { Expected } \\
\text { w/o cam } \\
\text { (EB } \\
\text { estimate) } \\
\end{array}$ & $\begin{array}{l}\text { Observed } \\
\% \text { change } \\
\end{array}$ & $\begin{array}{l}\text { \% change } \\
\text { (expected } \\
\& \\
\text { observed } \\
\text { after) } \\
\end{array}$ \\
\hline 1 & BRONSON AVE \& CARLING AVE & 43,684 & 4.0 & 0.6 & -0.3538 & -0.03 & 4.1 & $-85 \%$ & $-85 \%$ \\
\hline 2 & CARLING AVE \& RICHMOND RD & 46,299 & 3.0 & 3.5 & -0.3538 & -0.03 & 3.1 & $18 \%$ & $14 \%$ \\
\hline 3 & COVENTRY RD \& ST. LAURENT BLVD & 52,562 & 7.5 & 4.3 & -0.3538 & -0.03 & 7.8 & $-43 \%$ & $-45 \%$ \\
\hline 4 & EAGLESON RD \& HAZELDEAN RD & 37,775 & 2.5 & 1.6 & -0.3538 & -0.03 & 2.6 & $-37 \%$ & $-39 \%$ \\
\hline 5 & HERON RD \& RIVERSIDE DR & 58,882 & 7.0 & 2.8 & -0.3538 & -0.03 & 7.3 & $-60 \%$ & $-61 \%$ \\
\hline 6 & MEADOWLANDS DR \& MERIVALE RD & 47,966 & 3.5 & 1.3 & -0.3538 & -0.03 & 3.6 & $-62 \%$ & $-63 \%$ \\
\hline 7 & ALBERT ST \& KENT ST (OTTAWA) & 50,000 & 10.5 & 9.6 & -0.3538 & -0.03 & 10.9 & $-9 \%$ & $-12 \%$ \\
\hline 8 & BANK ST \& HUNT CLUB RD & 50,684 & 5.0 & 3.1 & -0.3538 & -0.03 & 5.2 & $-38 \%$ & $-40 \%$ \\
\hline 9 & BANK ST \& RIVERSIDE DR N & 41,598 & 3.3 & 3.9 & -0.3538 & -0.03 & 3.5 & $17 \%$ & $13 \%$ \\
\hline 10 & BASELINE RD \& MERIVALE RD & 46,821 & 5.1 & 3.1 & -0.3538 & -0.03 & 5.3 & $-39 \%$ & $-42 \%$ \\
\hline 11 & BELFAST RD \& ST. LAURENT BLVD & 38,197 & 3.3 & 3.4 & -0.3538 & -0.03 & 3.5 & $2 \%$ & $-2 \%$ \\
\hline 12 & BOOTH ST \& OTTAWA RIVER PKWY & 46,168 & 7.8 & 4.9 & -0.3538 & -0.03 & 8.1 & $-37 \%$ & $-39 \%$ \\
\hline 13 & CAMPEAU DR \& MARCH RD & 59,992 & 3.4 & 1.8 & -0.3538 & -0.03 & 3.6 & $-48 \%$ & $-50 \%$ \\
\hline 14 & CARLING AVE \& ISLAND PARK DR & 23,533 & 3.0 & 4.9 & -0.3538 & -0.03 & 3.1 & $63 \%$ & $57 \%$ \\
\hline 15 & CHARLEMAGNE BLVD N \& TENTH LINE RD & 35,827 & 3.8 & 2.5 & -0.3538 & -0.03 & 3.9 & $-34 \%$ & $-36 \%$ \\
\hline 16 & CATHERINE ST \& HWY 417 O'CONN & 50,000 & 11.8 & 5.4 & -0.3538 & -0.03 & 12.2 & $-54 \%$ & $-56 \%$ \\
\hline 17 & CATHERINE ST \& KENT ST & 50,000 & 5.8 & 7.3 & -0.3538 & -0.03 & 6.0 & $26 \%$ & $22 \%$ \\
\hline 18 & RUSSELL RD \& WALKLEY RD & 34,805 & 2.6 & 1.4 & -0.3538 & -0.03 & 2.7 & $-45 \%$ & $-47 \%$ \\
\hline 19 & ALBERT ST \& BOOTH ST & 27,558 & 5.4 & 4.5 & -0.3538 & -0.03 & 5.6 & $-16 \%$ & $-19 \%$ \\
\hline 20 & AVIATION PKWY \& OGILVIE RD & 40,664 & 3.7 & 1.8 & -0.3538 & -0.03 & 3.9 & $-53 \%$ & $-55 \%$ \\
\hline 21 & BANK ST \& HERON RD & 56,511 & 2.3 & 1.3 & -0.3538 & -0.03 & 2.4 & $-45 \%$ & $-47 \%$ \\
\hline
\end{tabular}




\begin{tabular}{|c|c|c|c|c|c|c|c|c|c|}
\hline 22 & BERRIGAN DR \& GREENBANK RD & 25,292 & 2.4 & 1.5 & -0.3538 & -0.03 & 2.5 & $-37 \%$ & $-39 \%$ \\
\hline 23 & BLAIR RD \& BLAIR RD RAMP 36 & 30,969 & 3.0 & 1.3 & -0.3538 & -0.03 & 3.1 & $-58 \%$ & $-60 \%$ \\
\hline 24 & BRONSON AVE \& COMMISSIONER ST & 18,746 & 1.7 & 1.5 & -0.3538 & -0.03 & 1.8 & $-13 \%$ & $-17 \%$ \\
\hline 25 & CONROY RD \& LORRY GREENBERG DR & 22,615 & 1.2 & 0.3 & -0.3538 & -0.03 & 1.2 & $-79 \%$ & $-80 \%$ \\
\hline 26 & CYRVILLE RD \& INNES RD & 55,925 & 1.5 & 1.0 & -0.3538 & -0.03 & 1.6 & $-35 \%$ & $-38 \%$ \\
\hline 27 & DRIVEWAY (THE) \& ISLAND PARK DR & 19,583 & 2.9 & 2.0 & -0.3538 & -0.03 & 3.0 & $-31 \%$ & $-34 \%$ \\
\hline 28 & DUFORD DR \& ST. JOSEPH BLVD & 25,612 & 3.6 & 4.3 & -0.3538 & -0.03 & 3.8 & $17 \%$ & $13 \%$ \\
\hline 29 & FISHER AVE \& MEADOWLANDS DR & 23,176 & 4.4 & 2.8 & -0.3538 & -0.03 & 4.5 & $-37 \%$ & $-39 \%$ \\
\hline 30 & $\begin{array}{l}\text { HAWTHORNE AVE \& QUEEN ELIZABETH } \\
\text { DRWY }\end{array}$ & 27,065 & 2.8 & 2.8 & -0.3538 & -0.03 & 2.9 & $-2 \%$ & $-6 \%$ \\
\hline 31 & JOCKVALE RD \& STRANDHERD DR & 17,536 & 3.7 & 3.8 & -0.3538 & -0.03 & 3.9 & $1 \%$ & $-3 \%$ \\
\hline 32 & KING EDWARD AVE \& ST. PATRICK ST & 33,216 & 8.0 & 1.0 & -0.3538 & -0.03 & 8.3 & $-88 \%$ & $-88 \%$ \\
\hline 33 & SAUNDERSON DR \& SMYTH RD & 49,546 & 1.7 & 0.5 & -0.3538 & -0.03 & 1.7 & $-70 \%$ & $-71 \%$ \\
\hline 34 & KING EDWARD AVE \& ST. ANDREW ST & 47,702 & 0.3 & 0.0 & -0.3538 & -0.03 & 0.4 & $-100 \%$ & $-100 \%$ \\
\hline & Total & & 142 & 95 & & & 147 & $-33 \%$ & $-53.83 \%$ \\
\hline
\end{tabular}

\begin{tabular}{|c|c|c|c|c|c|c|c|c|c|}
\hline & \multicolumn{9}{|c|}{ TURNING MOVEMENT COLLISIONS } \\
\hline & Intersection & AADT & $\begin{array}{l}\text { Average } \\
\text { observed } \\
\text { TurningMvmt } \\
\text { collisions/yr } \\
\text { BEFORE } \\
\text { Cam }\end{array}$ & $\begin{array}{l}\text { Average } \\
\text { observed } \\
\text { TurningMvmt } \\
\text { AFTER cam } \\
\text { coll/yr }\end{array}$ & $\begin{array}{l}\text { Predicted } \\
\text { w/o cam } \\
\text { using SPF } \\
\text { Model }\end{array}$ & weight & $\begin{array}{l}\text { Expected } \\
\text { w/o cam } \\
\text { (EB } \\
\text { estimate) }\end{array}$ & $\begin{array}{l}\text { Observed } \\
\% \text { change }\end{array}$ & $\begin{array}{l}\% \text { change } \\
\text { (expected } \\
\& \\
\text { observed } \\
\text { after) }\end{array}$ \\
\hline 1 & BRONSON AVE \& CARLING AVE & 43,684 & 6.0 & 5.4 & -0.4173 & -0.04 & 6.2 & $-10 \%$ & $-14 \%$ \\
\hline 2 & CARLING AVE \& RICHMOND RD & 46,299 & 1.0 & 0.6 & -0.4173 & -0.04 & 1.1 & $-38 \%$ & $-42 \%$ \\
\hline 3 & COVENTRY RD \& ST. LAURENT BLVD & 52,562 & 2.0 & 1.1 & -0.4173 & -0.04 & 2.1 & $-46 \%$ & $-48 \%$ \\
\hline 4 & EAGLESON RD \& HAZELDEAN RD & 37,775 & 2.0 & 0.8 & -0.4173 & -0.04 & 2.1 & $-58 \%$ & $-60 \%$ \\
\hline 5 & HERON RD \& RIVERSIDE DR & 58,882 & 1.5 & 1.8 & -0.4173 & -0.04 & 1.6 & $17 \%$ & $11 \%$ \\
\hline 6 & MEADOWLANDS DR \& MERIVALE RD & 47,966 & 5.0 & 8.0 & -0.4173 & -0.04 & 5.2 & $60 \%$ & $54 \%$ \\
\hline 7 & ALBERT ST \& KENT ST (OTTAWA) & 50,000 & 0.0 & 0.6 & -0.4173 & -0.04 & 0.0 & $0 \%$ & $3605 \%$ \\
\hline
\end{tabular}




\begin{tabular}{|c|c|c|c|c|c|c|c|c|c|}
\hline 8 & CARLING AVE \& ISLAND PARK DR & 23,533 & 1.3 & 3.2 & -0.4173 & -0.04 & 1.4 & $140 \%$ & $129 \%$ \\
\hline 9 & CHARLEMAGNE BLVD N \& TENTH LINE RD & 35,827 & 5.3 & 0.5 & -0.4173 & -0.04 & 5.6 & $-91 \%$ & $-91 \%$ \\
\hline 10 & BASELINE RD \& MERIVALE RD & 46,821 & 3.1 & 2.4 & -0.4173 & -0.04 & 3.2 & $-23 \%$ & $-26 \%$ \\
\hline 11 & BANK ST \& HUNT CLUB RD & 50,684 & 1.7 & 2.2 & -0.4173 & -0.04 & 1.7 & $32 \%$ & $26 \%$ \\
\hline 12 & BELFAST RD \& ST. LAURENT BLVD & 38,197 & 1.3 & 1.2 & -0.4173 & -0.04 & 1.4 & $-10 \%$ & $-14 \%$ \\
\hline 13 & BOOTH ST \& OTTAWA RIVER PKWY & 46,168 & 8.9 & 5.6 & -0.4173 & -0.04 & 9.2 & $-37 \%$ & $-39 \%$ \\
\hline 14 & CAMPEAU DR \& MARCH RD & 59,992 & 4.0 & 6.0 & -0.4173 & -0.04 & 4.2 & $50 \%$ & $44 \%$ \\
\hline 15 & BANK ST \& RIVERSIDE DR N & 41,598 & 1.8 & 1.9 & -0.4173 & -0.04 & 1.9 & $7 \%$ & $2 \%$ \\
\hline 16 & CATHERINE ST \& HWY 417 O'CONN & 50,000 & 0.1 & 0.6 & -0.4173 & -0.04 & 0.1 & $440 \%$ & $100 \%$ \\
\hline 17 & CATHERINE ST \& KENT ST & 50,000 & 9.4 & 4.7 & -0.4173 & -0.04 & 9.8 & $-50 \%$ & $-52 \%$ \\
\hline 18 & RUSSELL RD \& WALKLEY RD & 34,805 & 1.7 & 4.5 & -0.4173 & -0.04 & 1.7 & $170 \%$ & $158 \%$ \\
\hline 19 & $\begin{array}{l}\text { HAWTHORNE AVE \& QUEEN ELIZABETH } \\
\text { DRWY }\end{array}$ & 27,065 & 4.9 & 2.0 & -0.4173 & -0.04 & 5.1 & $-59 \%$ & $-61 \%$ \\
\hline 20 & JOCKVALE RD \& STRANDHERD DR & 17,536 & 5.4 & 5.0 & -0.4172 & -0.04 & 5.6 & $-7 \%$ & $-10 \%$ \\
\hline 21 & CYRVILLE RD \& INNES RD & 55,925 & 2.4 & 1.8 & -0.4173 & -0.04 & 2.5 & $-26 \%$ & $-29 \%$ \\
\hline 22 & ALBERT ST \& BOOTH ST & 27,558 & 7.5 & 3.5 & -0.4173 & -0.04 & 7.8 & $-54 \%$ & $-55 \%$ \\
\hline 23 & BRONSON AVE \& COMMISSIONER ST & 18,746 & 4.5 & 2.0 & -0.4172 & -0.04 & 4.6 & $-55 \%$ & $-57 \%$ \\
\hline 24 & CONROY RD \& LORRY GREENBERG DR & 22,615 & 0.9 & 2.3 & -0.4173 & -0.04 & 1.0 & $148 \%$ & $135 \%$ \\
\hline 25 & DRIVEWAY (THE) \& ISLAND PARK DR & 19,583 & 0.7 & 0.8 & -0.4172 & -0.04 & 0.8 & $3 \%$ & $-3 \%$ \\
\hline 26 & DUFORD DR \& ST. JOSEPH BLVD & 25,612 & 1.1 & 1.5 & -0.4173 & -0.04 & 1.1 & $38 \%$ & $31 \%$ \\
\hline 27 & FISHER AVE \& MEADOWLANDS DR & 23,176 & 0.9 & 1.3 & -0.4173 & -0.04 & 1.0 & $38 \%$ & $30 \%$ \\
\hline 28 & BANK ST \& HERON RD & 56,511 & 1.6 & 0.5 & -0.4173 & -0.04 & 1.7 & $-69 \%$ & $-71 \%$ \\
\hline 29 & BERRIGAN DR \& GREENBANK RD & 25,292 & 2.9 & 4.5 & -0.4173 & -0.04 & 3.0 & $55 \%$ & $48 \%$ \\
\hline 30 & BLAIR RD \& BLAIR RD RAMP 36 & 30,969 & 4.5 & 3.8 & -0.4173 & -0.04 & 4.6 & $-16 \%$ & $-19 \%$ \\
\hline 31 & AVIATION PKWY \& OGILVIE RD & 40,664 & 2.0 & 5.3 & -0.4173 & -0.04 & 2.1 & $163 \%$ & $151 \%$ \\
\hline 32 & SAUNDERSON DR \& SMYTH RD & 49,546 & 4.4 & 1.0 & -0.4173 & -0.04 & 4.6 & $-77 \%$ & $-78 \%$ \\
\hline 33 & KING EDWARD AVE \& ST. PATRICK ST & 33,216 & 1.4 & 0.5 & -0.4173 & -0.04 & 1.5 & $-65 \%$ & $-66 \%$ \\
\hline \multirow[t]{2}{*}{34} & KING EDWARD AVE \& ST. ANDREW ST & 47,702 & 0.2 & $\mathbf{0 . 0}$ & -0.4173 & -0.04 & 0.2 & $-100 \%$ & $-100 \%$ \\
\hline & Total & & 101 & 87 & & & 106 & $-15 \%$ & $-22.24 \%$ \\
\hline
\end{tabular}


SIDESWIPE COLLISIONS

\begin{tabular}{|c|c|c|c|c|c|c|c|c|c|}
\hline & Intersection & AADT & $\begin{array}{l}\text { Average } \\
\text { observed } \\
\text { Sideswipe } \\
\text { collisions/yr } \\
\text { BEFORE } \\
\text { Cam }\end{array}$ & $\begin{array}{l}\text { Average } \\
\text { observed } \\
\text { Sideswipe } \\
\text { AFTER cam } \\
\text { coll/yr }\end{array}$ & $\begin{array}{l}\text { Predicted } \\
\text { w/o cam } \\
\text { using SPF } \\
\text { Model }\end{array}$ & weight & $\begin{array}{l}\text { Expected } \\
\text { w/o cam } \\
\text { (EB } \\
\text { estimate) }\end{array}$ & $\begin{array}{l}\text { Observed } \\
\% \text { change }\end{array}$ & $\begin{array}{l}\text { \% change } \\
\text { (expected } \\
\& \\
\text { observed } \\
\text { after) }\end{array}$ \\
\hline 1 & BRONSON AVE \& CARLING AVE & 43,684 & 0.7 & 2.0 & -0.5916 & -0.05 & 0.8 & $175 \%$ & $153 \%$ \\
\hline 2 & CARLING AVE \& RICHMOND RD & 46,299 & 0.0 & 0.8 & -0.5916 & -0.05 & 0.0 & \#DIV/0! & $2533 \%$ \\
\hline 3 & COVENTRY RD \& ST. LAURENT BLVD & 52,562 & 0.8 & 0.5 & -0.5916 & -0.05 & 0.9 & $-39 \%$ & $-44 \%$ \\
\hline 4 & EAGLESON RD \& HAZELDEAN RD & 37,775 & 4.8 & 6.0 & -0.5916 & -0.05 & 5.1 & $25 \%$ & $18 \%$ \\
\hline 5 & HERON RD \& RIVERSIDE DR & 58,882 & 3.0 & 5.0 & -0.5916 & -0.05 & 3.2 & $67 \%$ & $58 \%$ \\
\hline 6 & MEADOWLANDS DR \& MERIVALE RD & 47,966 & 2.0 & 2.6 & -0.5916 & -0.05 & 2.1 & $30 \%$ & $22 \%$ \\
\hline 7 & ALBERT ST \& KENT ST (OTTAWA) & 50,000 & 2.4 & 1.4 & -0.5916 & -0.05 & 2.6 & $\mathbf{0 \%}$ & $-46 \%$ \\
\hline 8 & CARLING AVE \& ISLAND PARK DR & 23,533 & 2.0 & 3.8 & -0.5916 & -0.05 & 2.1 & $90 \%$ & $79 \%$ \\
\hline 9 & CHARLEMAGNE BLVD N \& TENTH LINE RD & 35,827 & 0.4 & 0.3 & -0.5916 & -0.05 & 0.4 & $-31 \%$ & $-39 \%$ \\
\hline 10 & BASELINE RD \& MERIVALE RD & 46,821 & 0.5 & 1.0 & -0.5916 & -0.05 & 0.6 & $83 \%$ & $67 \%$ \\
\hline 11 & BANK ST \& HUNT CLUB RD & 50,684 & 0.1 & 1.4 & -0.5916 & -0.05 & 0.1 & $1160 \%$ & $866 \%$ \\
\hline 12 & BELFAST RD \& ST. LAURENT BLVD & 38,197 & 2.0 & 2.9 & -0.5916 & -0.05 & 2.1 & $46 \%$ & $38 \%$ \\
\hline 13 & BOOTH ST \& OTTAWA RIVER PKWY & 46,168 & 0.9 & 0.8 & -0.5916 & -0.05 & 1.0 & $-18 \%$ & $-24 \%$ \\
\hline 14 & CAMPEAU DR \& MARCH RD & 59,992 & 1.2 & 0.6 & -0.5916 & -0.05 & 1.3 & $-51 \%$ & $-54 \%$ \\
\hline 15 & BANK ST \& RIVERSIDE DR N & 41,598 & 0.3 & 1.4 & -0.5916 & -0.05 & 0.4 & $320 \%$ & $271 \%$ \\
\hline 16 & CATHERINE ST \& HWY 417 O'CONN & 50,000 & 0.0 & 1.5 & -0.5916 & -0.05 & 0.0 & \#DIV/0! & $100 \%$ \\
\hline 17 & CATHERINE ST \& KENT ST & 50,000 & 2.2 & 1.8 & -0.5916 & -0.05 & 2.4 & $-19 \%$ & $-24 \%$ \\
\hline 18 & RUSSELL RD \& WALKLEY RD & 34,805 & 4.1 & 5.0 & -0.5916 & -0.05 & 4.3 & $22 \%$ & $15 \%$ \\
\hline 19 & $\begin{array}{l}\text { HAWTHORNE AVE \& QUEEN ELIZABETH } \\
\text { DRWY }\end{array}$ & 27,065 & 0.4 & 0.8 & -0.5916 & -0.05 & 0.5 & $80 \%$ & $62 \%$ \\
\hline 20 & JOCKVALE RD \& STRANDHERD DR & 17,536 & 0.3 & $\mathbf{0 . 0}$ & -0.5915 & -0.05 & 0.3 & $-100 \%$ & $-100 \%$ \\
\hline 21 & CYRVILLE RD \& INNES RD & 55,925 & 3.5 & 4.5 & -0.5916 & -0.05 & 3.7 & $29 \%$ & $22 \%$ \\
\hline 22 & ALBERT ST \& BOOTH ST & 27,558 & 1.6 & 1.3 & -0.5916 & -0.05 & 1.7 & $-24 \%$ & $-28 \%$ \\
\hline 23 & BRONSON AVE \& COMMISSIONER ST & 18,746 & 0.2 & 0.5 & -0.5915 & -0.05 & 0.2 & $175 \%$ & $128 \%$ \\
\hline 24 & CONROY RD \& LORRY GREENBERG DR & 22,615 & 0.3 & 0.5 & -0.5916 & -0.05 & 0.3 & $83 \%$ & $59 \%$ \\
\hline 25 & DRIVEWAY (THE) \& ISLAND PARK DR & 19,583 & 0.5 & 1.3 & -0.5915 & -0.05 & 0.6 & $150 \%$ & $126 \%$ \\
\hline
\end{tabular}




\begin{tabular}{|c|c|c|c|c|c|c|c|c|c|}
\hline 26 & DUFORD DR \& ST. JOSEPH BLVD & 25,612 & 0.7 & 1.5 & -0.5916 & -0.05 & 0.8 & $106 \%$ & $90 \%$ \\
\hline 27 & FISHER AVE \& MEADOWLANDS DR & 23,176 & 0.4 & 0.3 & -0.5916 & -0.05 & 0.4 & $-31 \%$ & $-39 \%$ \\
\hline 28 & BANK ST \& HERON RD & 56,511 & 1.0 & 1.8 & -0.5916 & -0.05 & 1.1 & $75 \%$ & $63 \%$ \\
\hline 29 & BERRIGAN DR \& GREENBANK RD & 25,292 & 0.1 & 0.0 & -0.5916 & -0.05 & 0.1 & $-100 \%$ & $-100 \%$ \\
\hline 30 & BLAIR RD \& BLAIR RD RAMP 36 & 30,969 & 0.2 & 0.0 & -0.5916 & -0.05 & 0.2 & $-100 \%$ & $-100 \%$ \\
\hline 31 & AVIATION PKWY \& OGILVIE RD & 40,664 & 5.3 & 2.5 & -0.5916 & -0.05 & 5.5 & $-52 \%$ & $-55 \%$ \\
\hline 32 & SAUNDERSON DR \& SMYTH RD & 49,546 & 1.0 & 2.0 & -0.5916 & -0.05 & 1.1 & $100 \%$ & $86 \%$ \\
\hline 33 & KING EDWARD AVE \& ST. PATRICK ST & 33,216 & 2.3 & 5.6 & -0.5916 & -0.05 & 2.5 & $140 \%$ & $126 \%$ \\
\hline 34 & KING EDWARD AVE \& ST. ANDREW ST & 47,702 & 0.2 & 0.5 & -0.5916 & -0.05 & 0.2 & $200 \%$ & $146 \%$ \\
\hline & Total & & 46 & 62 & & & 49 & $35 \%$ & $20.87 \%$ \\
\hline
\end{tabular}




\section{Appendix K - PSM Output}

\section{TOTAL COLLISIONS}

- teffects ipwra (totalcollisions lnaadt median channelization leftturn thrulanes fplt plt pplt speed1 speed2) (L1rlcinstalled2 L1rearend Llangle L1TM ), atet

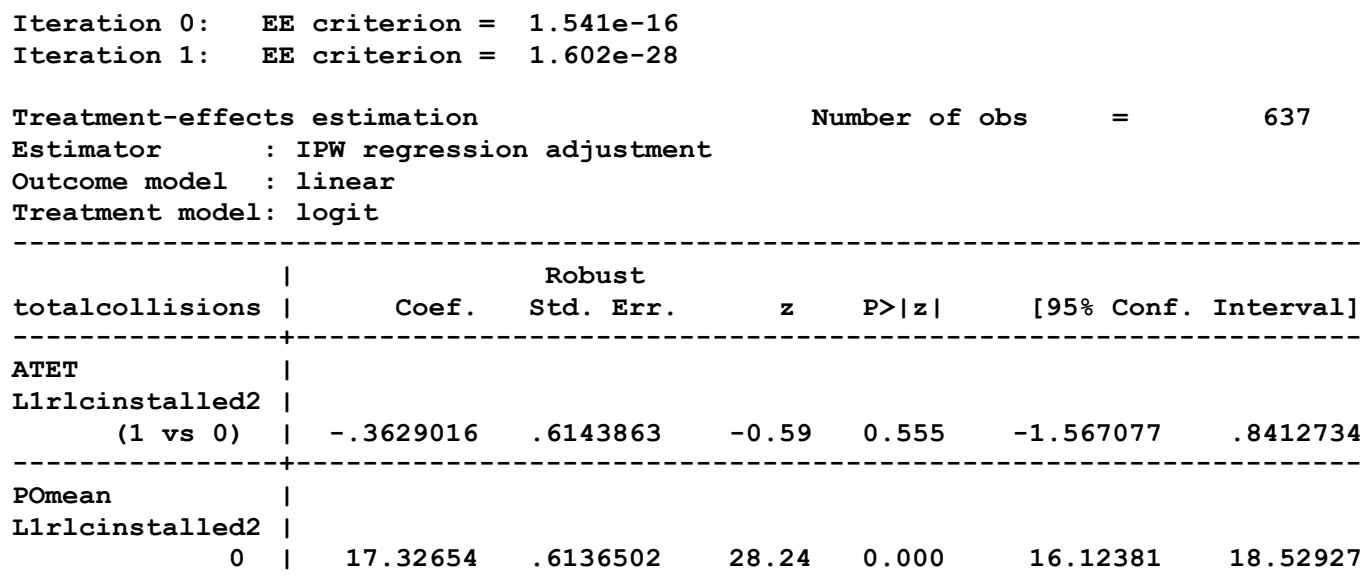

* *with lags - outcome NOT repeated in treamtment

- tebalance overid

Iteration 0: criterion $=.13620852$

Iteration 1: criterion $=.13748158$ (backed up)

Iteration 2: criterion $=.13755498$ (backed up)

Iteration 3: $\quad$ criterion $=.13758405$

Iteration $4:$ criterion $=.13816077$

Iteration 5: criterion $=.13838816$

Iteration $6:$ criterion $=.13838825$

Overidentification test for covariate balance

H0: Covariates are balanced:

$$
\begin{array}{llr}
\text { chi2 (4) } & =6.22605 \\
\text { Prob > chi2 } & 0.1829
\end{array}
$$

- psmatch2 rlcinstalled2 lnaadt median channelization leftturn thrulanes fplt plt pplt speed1

> speed2, logit common outcome (totalcollisions) ties

Logistic regression

$\begin{array}{llr}\text { Number of obs } & = & 685 \\ \text { LR chi2(10) } & = & 84.15 \\ \text { Prob > chi2 } & = & 0.0000 \\ \text { Pseudo R2 } & = & 0.1019\end{array}$

Log likelihood $=-370.71141$

Pseudo R2

\begin{tabular}{|c|c|c|c|c|c|c|c|}
\hline rlcinstalled2 & I & Coef. & Std. Err. & $\mathbf{z}$ & $P>|z|$ & [95\% Conf. & Interval] \\
\hline--- & + & --- & - & & -- & - & -- \\
\hline Inaadt & l & 5202112 & .2599367 & 2.00 & 0.045 & .0107447 & 1.029678 \\
\hline median & I & -1.045775 & .2579872 & -4.05 & 0.000 & -1.551421 & -.5401296 \\
\hline channelization & i & .4969581 & .2293971 & 2.17 & 0.030 & .0473481 & .946568 \\
\hline leftturn & i & .549823 & .1609388 & 3.42 & 0.001 & .2343887 & .8652573 \\
\hline thrulanes & i & .8612656 & .1567287 & 5.50 & 0.000 & .5540831 & 1. 168448 \\
\hline fplt & i & -1.328941 & .3086871 & -4.31 & 0.000 & -1.933957 & -.7239258 \\
\hline plt & i & -1.384477 & .31272 & -4.43 & 0.000 & -1.997397 & -.7715569 \\
\hline pplt & i & -1.390921 & .3041415 & -4.57 & 0.000 & -1.987028 & -.794815 \\
\hline speed1 & i & -.0124766 & .011701 & -1.07 & 0.286 & -.0354101 & .010457 \\
\hline speed2 & I & .0031362 & .015014 & 0.21 & 0.835 & -.0262907 & .0325631 \\
\hline
\end{tabular}

[95\% Conf. Interval] 


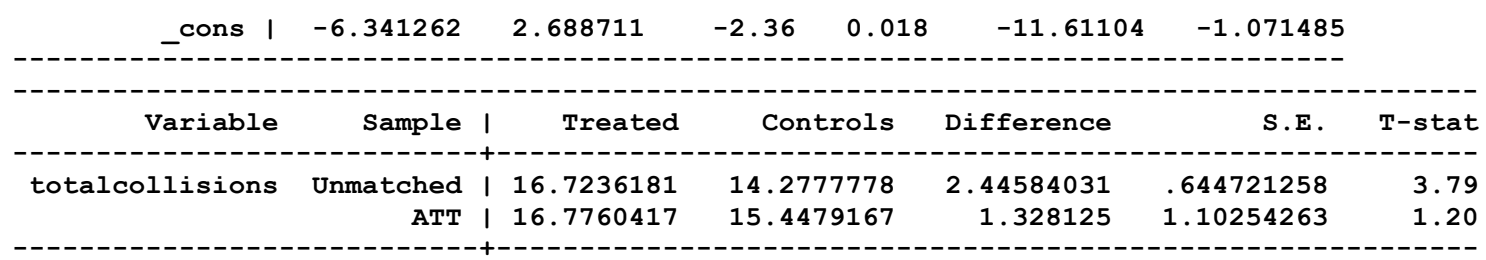

Note: S.E. does not take into account that the propensity score is estimated.

\begin{tabular}{r|rrr} 
psmatch2: | & psmatch2: Common \\
Treatment | & support & \\
assignment | Off suppo On suppor | & Total \\
\hline-0 & 0 & 486 & 486 \\
Untreated | & 7 & 192 & 199 \\
Treated | & 7 & 678 & 685
\end{tabular}

end of do-file

. do "C: \Users\Amal\AppData\Local\Temp\STD04000000. tmp"

- pstest lnaadt median channelization leftturn thrulanes fplt plt pplt speed1 speed2, both gra

$>\mathrm{ph}$

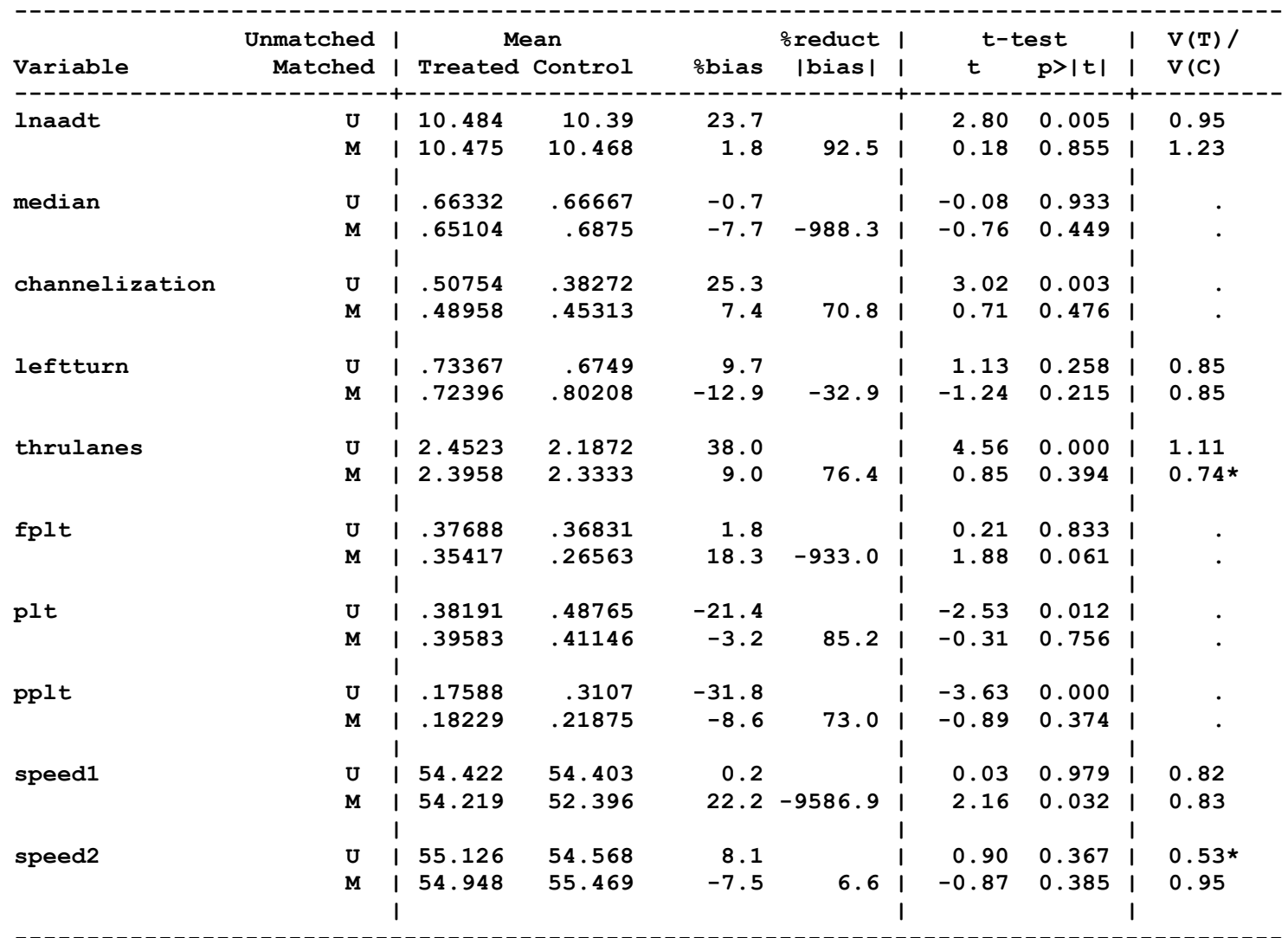

* if variance ratio outside $[0.76 ; 1.32]$ for $U$ and $[0.75 ; 1.33]$ for $M$

\begin{tabular}{|c|c|c|c|c|c|c|c|c|}
\hline Sample & I Ps R2 & LR chi2 & $p>\operatorname{chi} 2$ & MeanBias & MedBias & B & $\mathbf{R}$ & \%Var \\
\hline & & & & & & & & \\
\hline Unmatched & 10.105 & 86.28 & 0.000 & 16.1 & 15.6 & 79.4 * & 0.32 * & 20 \\
\hline Matched & 10.030 & 15.92 & 0.102 & 9.8 & 8.2 & $41.2 \star$ & 0.71 & 20 \\
\hline
\end{tabular}


* if $B>25 \%, R$ outside $[0.5 ; 2]$

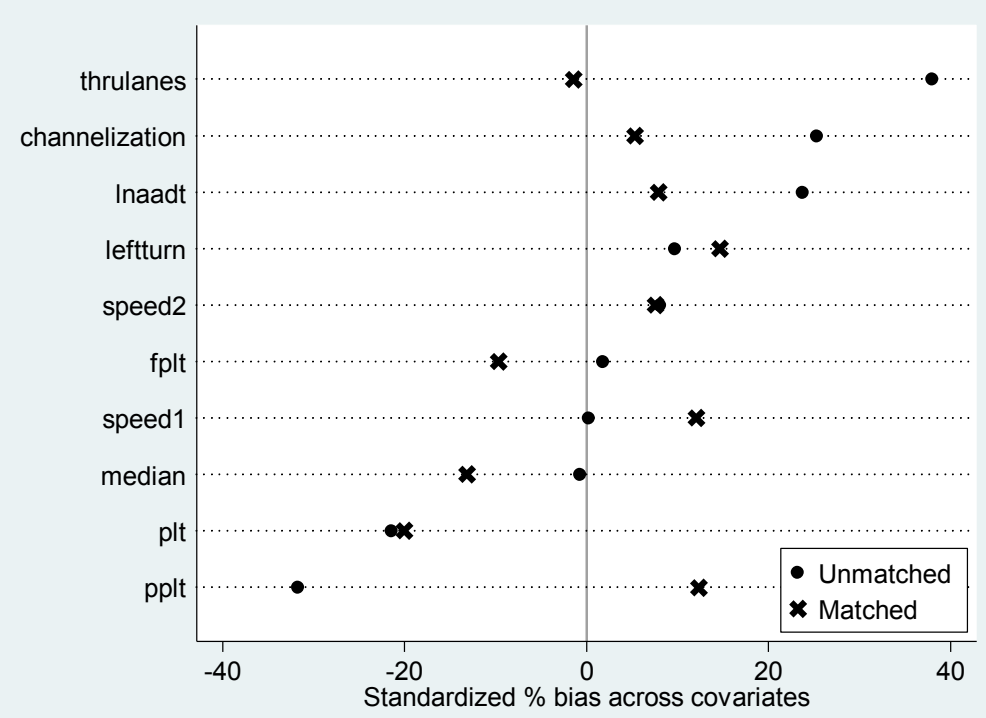

- pstest_pscore, density both

psmatch2: Propensity Score
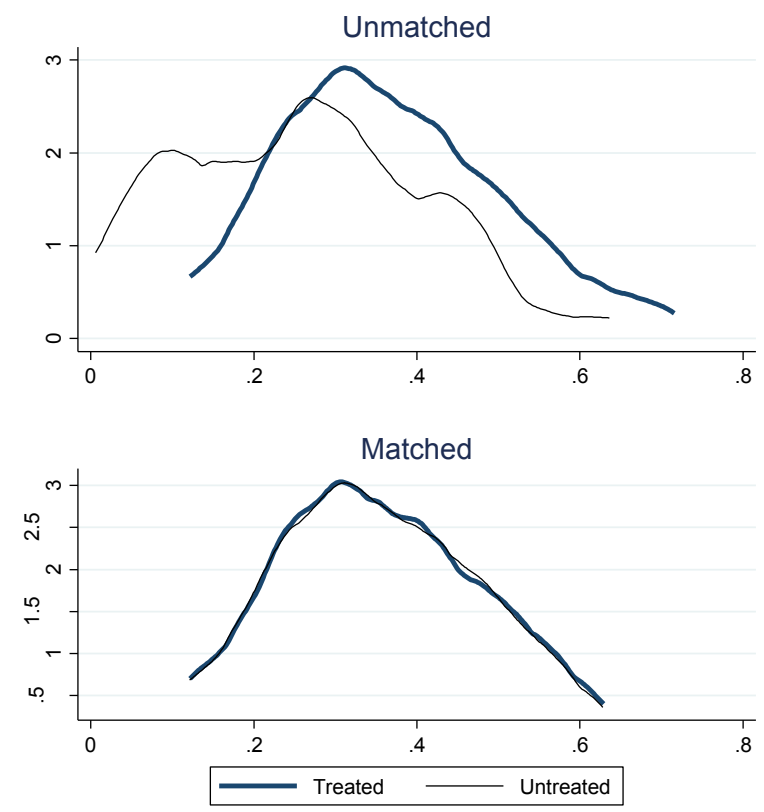

\section{TOTAL WITH SPILLOVER}

- psmatch2 rlcinstalled2 lnaadt median channelization leftturn thrulanes plt p $>$ plt closestrlc0to500 twoormorerlc0to500 threewithinrlc1000 numcams timehalo

$>$ yr timehalo timehalo2, logit common outcome (totalcollisions) ties 


\begin{tabular}{|c|c|c|c|c|c|c|}
\hline \multicolumn{4}{|c|}{ Log likelihood $=-195.89568$} & \multicolumn{2}{|c|}{$\begin{array}{l}\text { LR chi2 (14) } \\
\text { Prob > chi2 } \\
\text { Pseudo R2 }\end{array}$} & $\begin{array}{l}433.78 \\
0.0000 \\
0.5254\end{array}$ \\
\hline rlcinstall 2 | & Coef. & Std. Err. & $\mathbf{z}$ & $P>|z|$ & [95\% Conf. & Interval] \\
\hline \begin{tabular}{l|} 
lnaadt \\
median
\end{tabular} & $\begin{array}{r}.126625 \\
-1.601679\end{array}$ & $\begin{array}{l}.3708407 \\
.4001783\end{array}$ & $\begin{array}{r}0.34 \\
-4.00\end{array}$ & $\begin{array}{l}0.733 \\
0.000\end{array}$ & $\begin{array}{l}-.6002095 \\
-2.386014\end{array}$ & $\begin{array}{r}.8534594 \\
-.817344\end{array}$ \\
\hline channeliza n | & -.0455128 & .3338912 & -0.14 & 0.892 & -.6999275 & .6089019 \\
\hline leftturn 1 & -.2737637 & .2450188 & -1.12 & 0.264 & -.7539918 & .2064643 \\
\hline thrulanes 1 & 1.361598 & .2390263 & 5.70 & 0.000 & .8931152 & 1.830081 \\
\hline plt 1 & -.4718743 & .3284175 & -1.44 & 0.151 & -1.115561 & .1718123 \\
\hline pplt I & -2.604665 & 405621 & -6.42 & 0.000 & -3.399668 & -1.809663 \\
\hline coto500 I & -3.905904 & .471707 & -8.28 & 0.000 & -4.830433 & -2.981376 \\
\hline twoo c0to500 I & .8112562 & .70269 & 1.15 & 0.248 & -.5659909 & 2.188503 \\
\hline threewi 1000 | & -1.359991 & .6938614 & -1.96 & 0.050 & -2.719934 & -.0000476 \\
\hline numcams i & .1541738 & .0408894 & 3.77 & 0.000 & .074032 & .2343157 \\
\hline timehaloyr 1 & .1290936 & .103199 & 1.25 & 0.211 & -.0731727 & .3313599 \\
\hline timehalo I & 5903 & .6235035 & 0.95 & 0.344 & -.6317231 & 1.812366 \\
\hline timehalo2 & -.0979062 & .1450672 & -0.67 & 0.500 & -.3822327 & .1864203 \\
\hline _cons I & -5.500795 & 3.883157 & -1.42 & 0.157 & -13.11164 & 2.110053 \\
\hline
\end{tabular}

There are observations with identical propensity score values.

The sort order of the data could affect your results.

Make sure that the sort order is random before calling psmatch2.

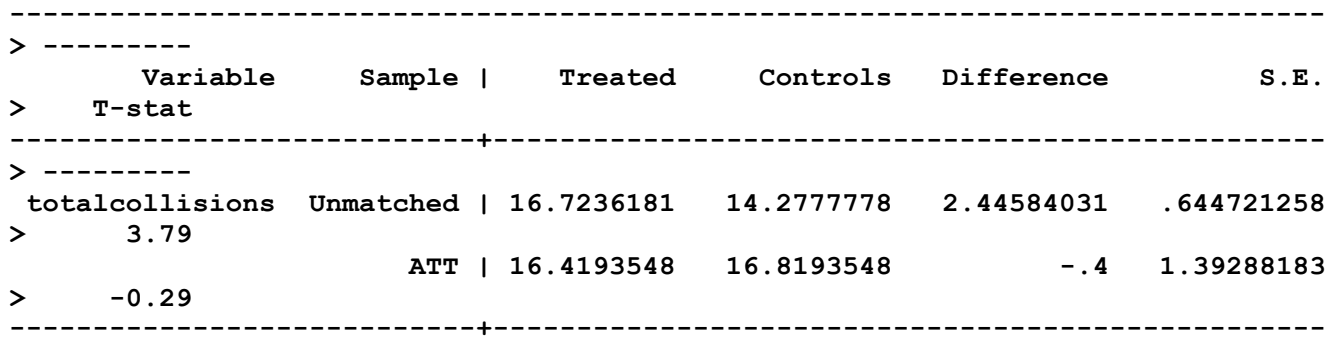

$>----1--$

Note: S.E. does not take into account that the propensity score is estimated.

\begin{tabular}{r|rrr} 
psmatch2: | & psmatch2: Common & \\
Treatment | & support & \\
assignment | Off suppo On suppor | & Total \\
\hline Untreated | & 0 & 486 & 486 \\
Treated | & 44 & 155 & 199 \\
\hline-20 & 44 & 641 & 685
\end{tabular}

- pstest lnaadt median channelization leftturn thrulanes plt pplt closestrlc0to $>500$ twoormorerlc0to500 threewithinrlc1000 numcams timehaloyr timehalo timeh $>$ alo2, both graph

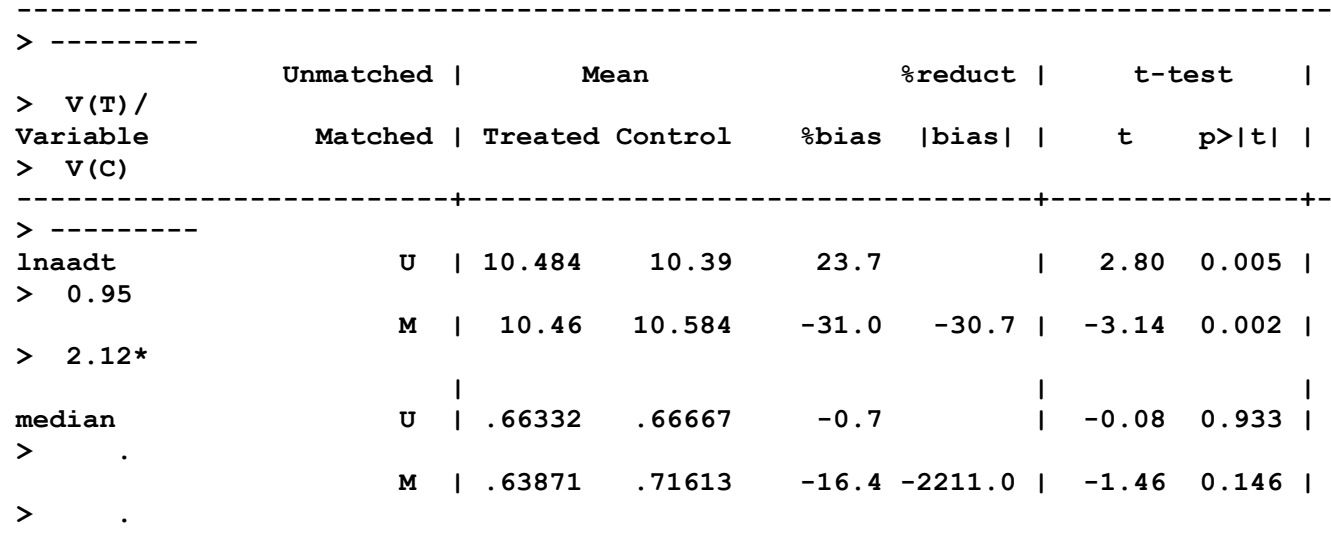




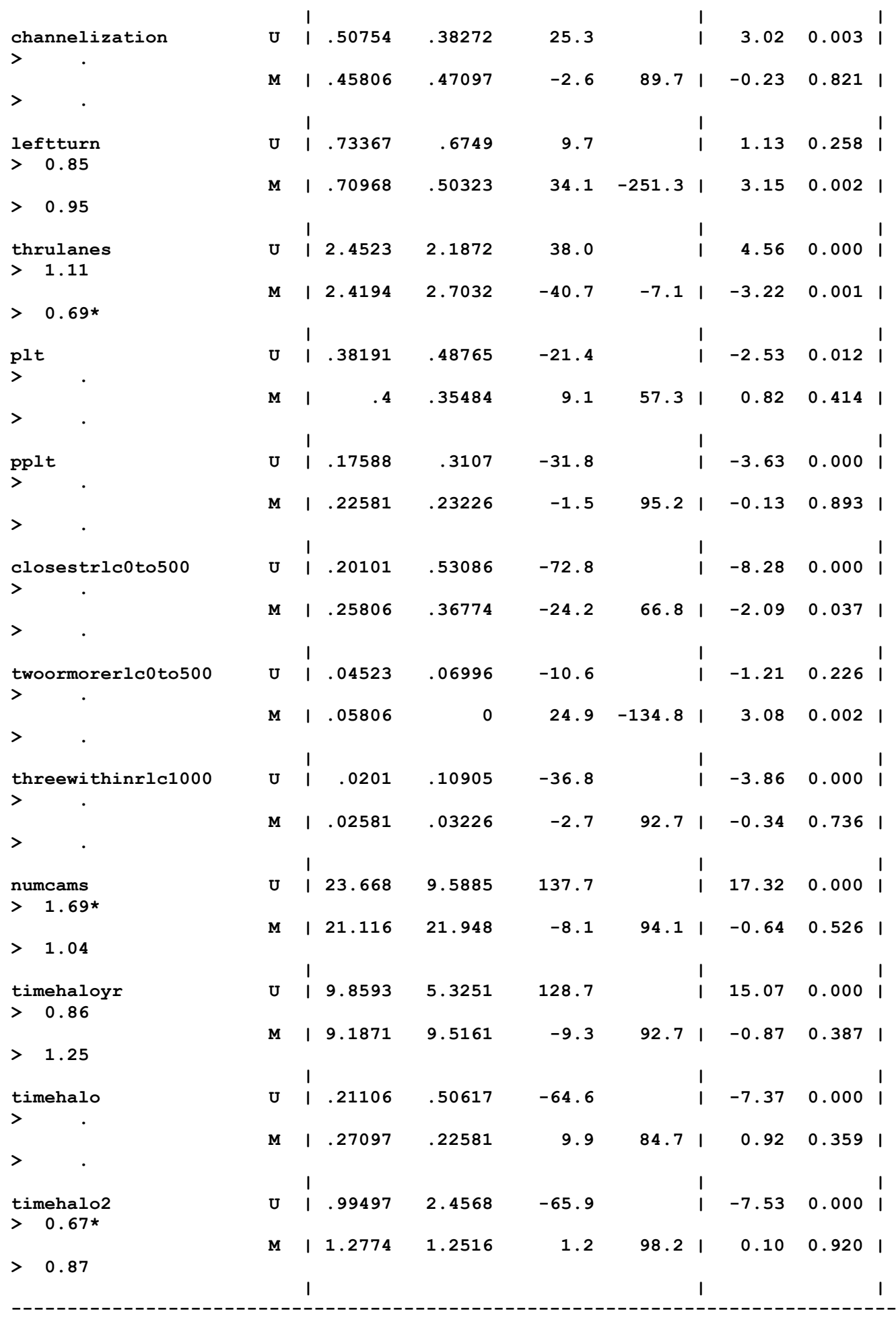

* if variance ratio outside $[0.76 ; 1.32]$ for $U$ and $[0.73 ; 1.37]$ for $M$

Sample I Ps R2 LR chi2 p>chi2 MeanBias MedBias $\quad$ B R R
$>$ ar
$>$
$>$




\begin{tabular}{|c|c|c|c|c|c|c|c|}
\hline $\begin{array}{l}\text { Unmatched } \\
>33\end{array}$ & I 0.527 & 435.40 & 0.000 & 47.7 & 34.3 & 212.2 * & 0.66 \\
\hline $\begin{array}{l}\text { Matched } \\
>33\end{array}$ & 10.084 & 35.17 & 0.001 & 15.4 & 9.6 & $70.9 *$ & 0.87 \\
\hline
\end{tabular}

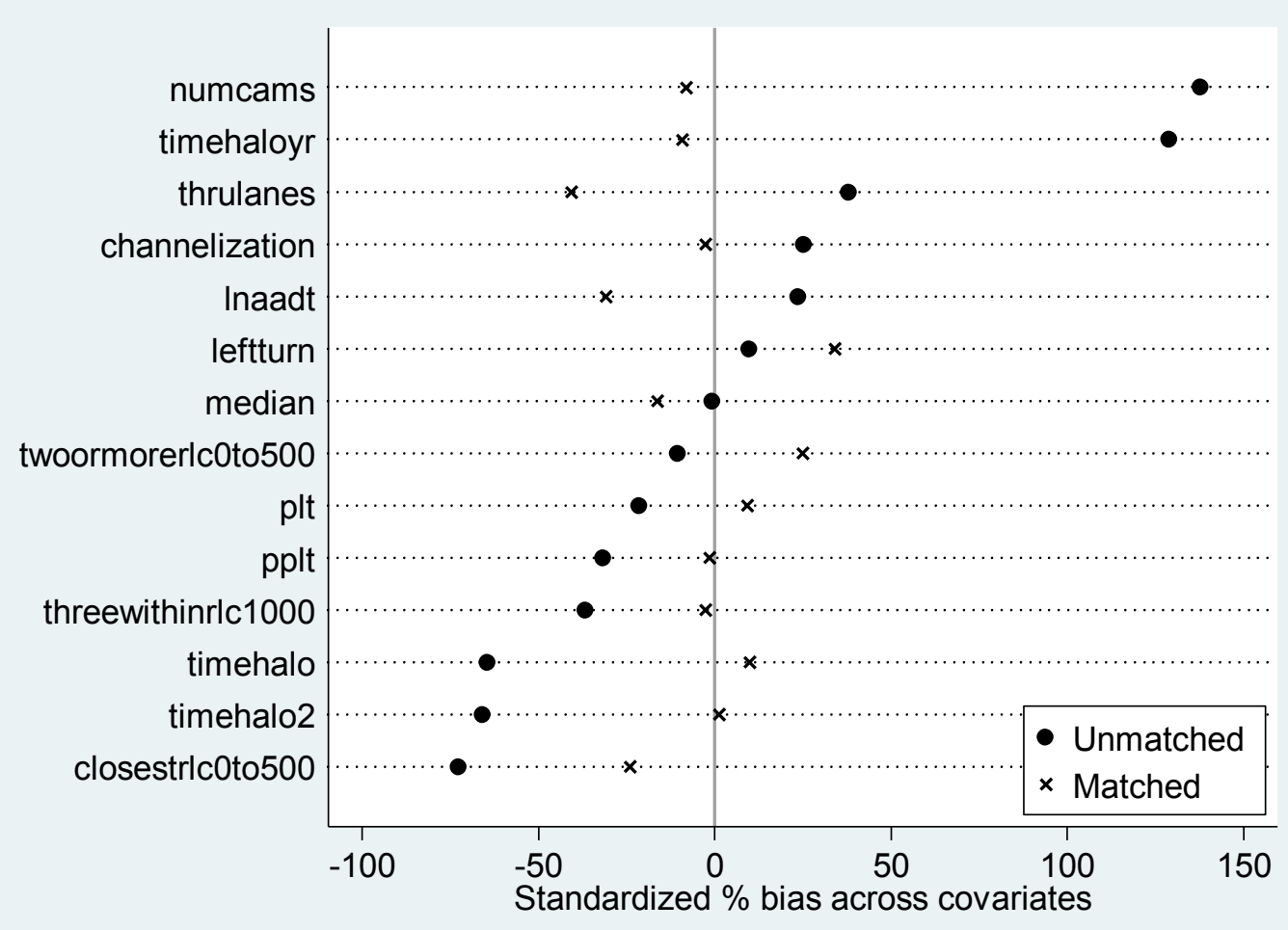

pstest pscore, density both 


\section{psmatch2: Propensity Score}
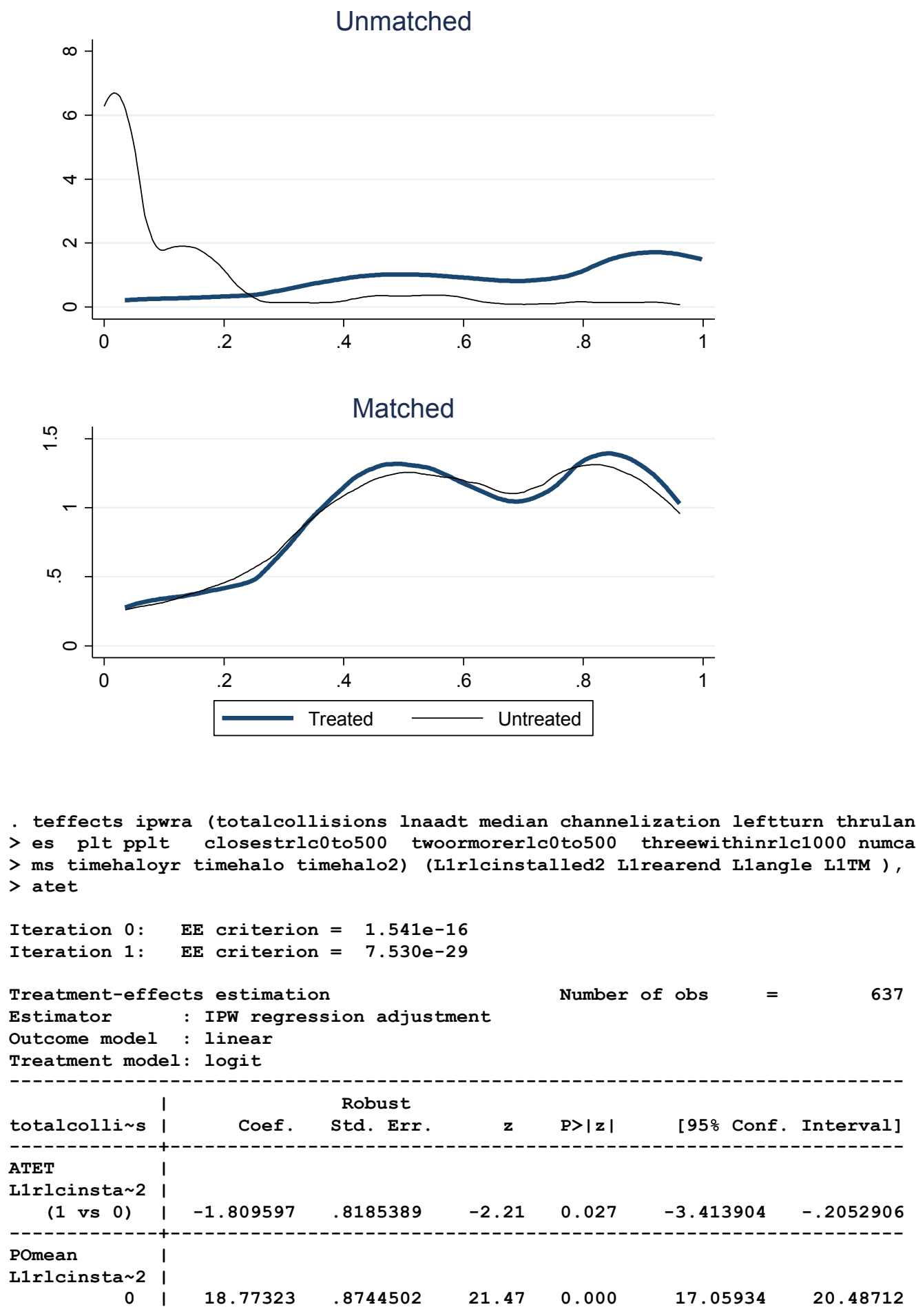

\section{. REAR-END}


- teffects ipwra (rearend lnaadt median channelization leftturn thrulanes fplt plt pplt speed1 speed2) (I1rlcinstalled2 L1total Llangle L1TM), atet

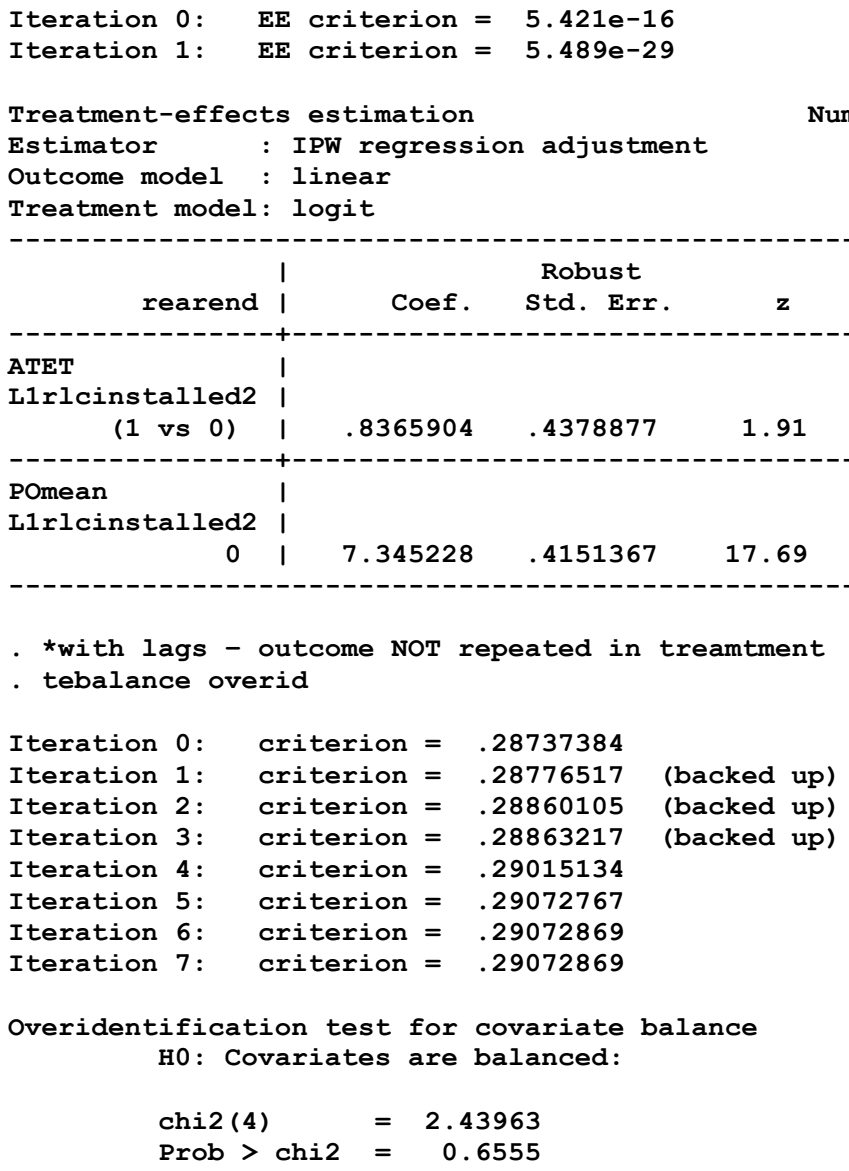

\section{ANGLE}

- teffects ipwra (angle lnaadt median channelization leftturn thrulanes fplt plt pplt speed1 speed2) (L1rlcinstalled2 L1total L1rearend L1TM), atet

Iteration 0: $\quad \mathrm{EE}$ criterion $=5.242 \mathrm{e}-16$

Iteration 1: $\quad \mathrm{EE}$ criterion $=3.217 \mathrm{e}-30$

$\begin{array}{lll}\text { Treatment-effects estimation } & \text { Number of obs } \quad=\quad 637\end{array}$

Estimator : IPW regression adjustment

Outcome model : linear

Treatment model: logit

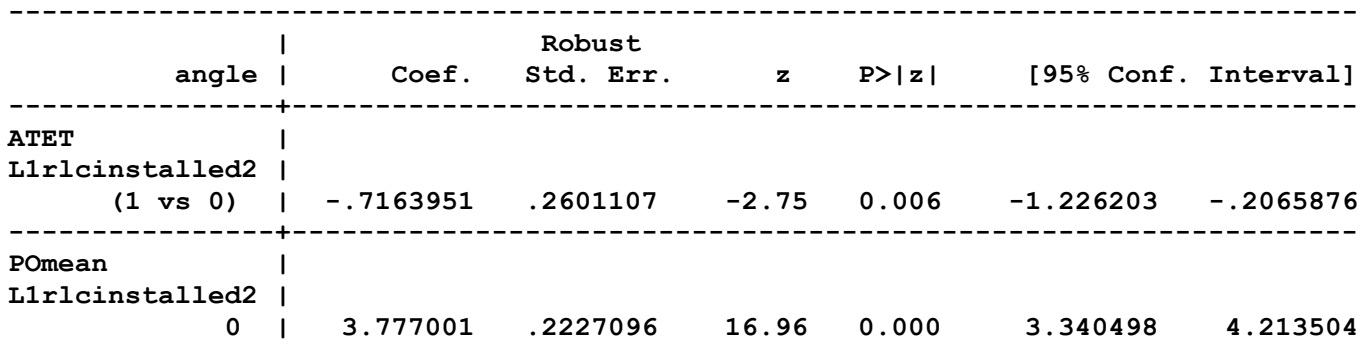

*with lags - outcome NOT repeated in treamtment

- tebalance overid 


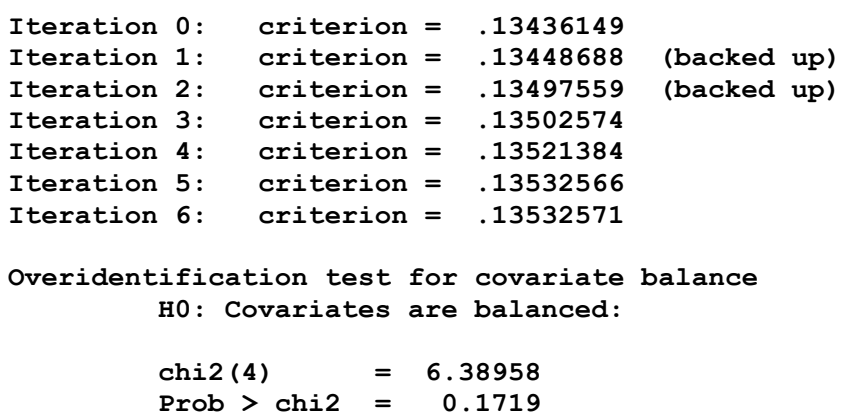

\section{TURNINGMOVEMENT}

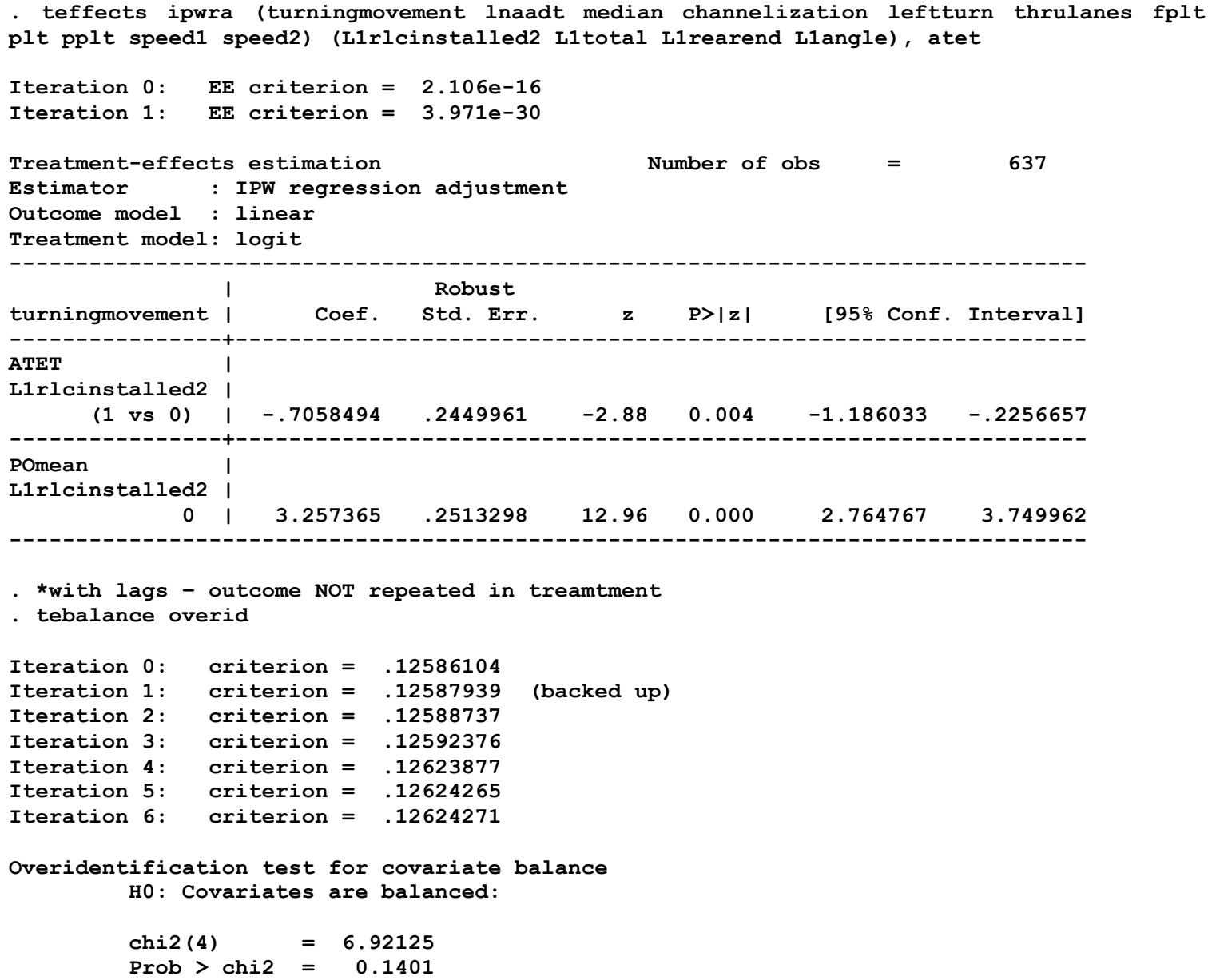

\section{SIDESWIPE}

teffects ipwra (sideswipe lnaadt median channelization leftturn thrulanes fplt plt pplt spee

> d1 speed2) (L1rlcinstalled2 L1total L1rearend Llangle L1TM ), atet

Iteration 0: $\mathrm{EE}$ criterion $=5.169 \mathrm{e}-16$

Iteration 1: $\quad \mathrm{EE}$ criterion $=7.580 \mathrm{e}-30$

Treatment-effects estimation $\quad$ Number of obs $=637$

Estimator : IPW regression adjustment 


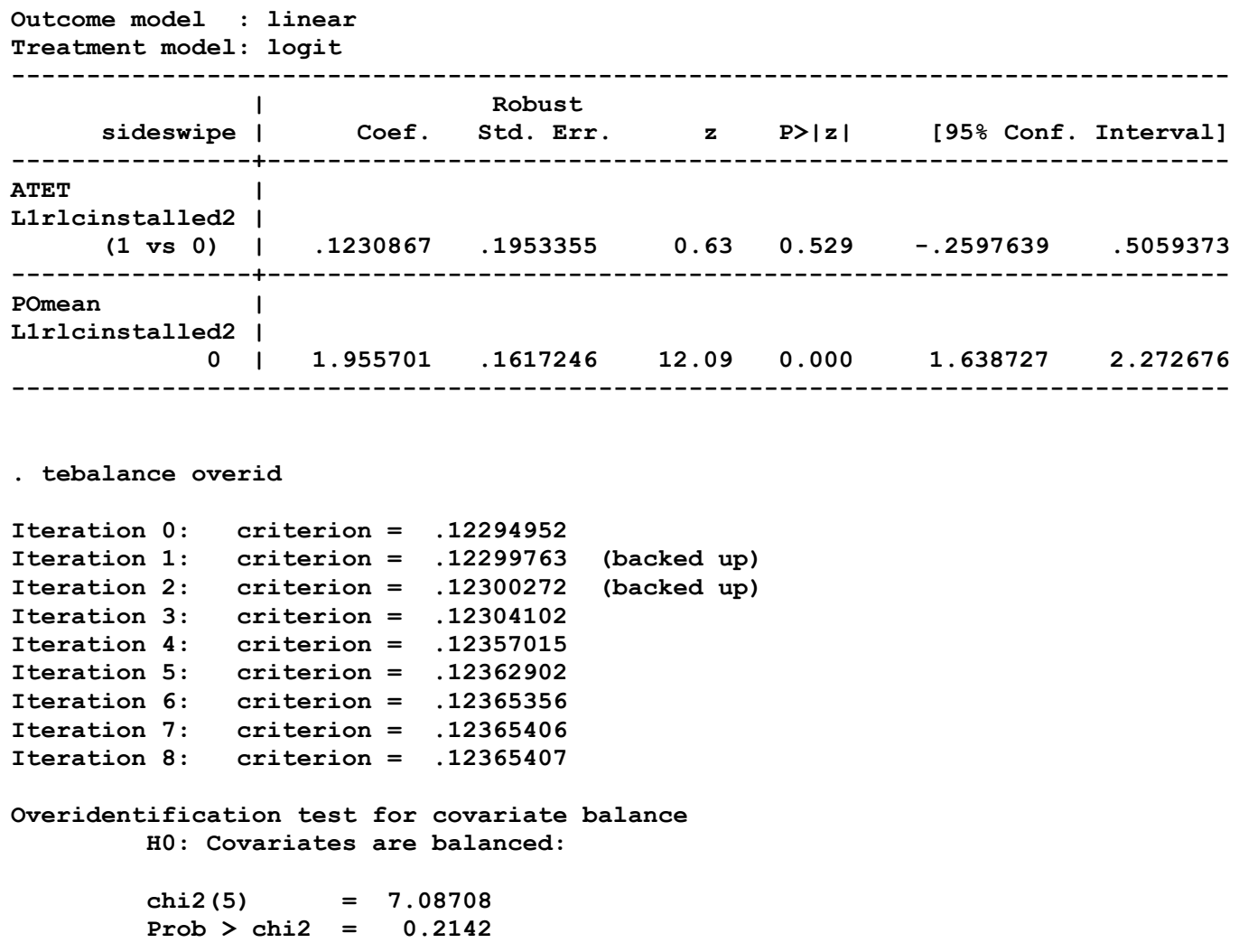


Appendix L - Sensitivity Analysis 


\section{HIGH COSTS}

\begin{tabular}{|c|c|c|c|c|c|c|c|c|}
\hline & & Annual & number of $\mathrm{c}$ & lisions & & 1ual cost of collis & ons & \\
\hline Collision Impact Type & $\begin{array}{l}\text { \# of RLC } \\
\text { sites }\end{array}$ & $\begin{array}{l}\text { Annual } \\
\text { number of } \\
\text { Fatal } \\
\text { collisions } \\
\text { after RLC }\end{array}$ & $\begin{array}{c}\text { Annual } \\
\text { number of } \\
\text { Injury } \\
\text { collisions } \\
\text { after RLC }\end{array}$ & $\begin{array}{c}\text { Annual } \\
\text { number of } \\
\text { PDO } \\
\text { collisions } \\
\text { after RLC }\end{array}$ & $\begin{array}{l}\text { Cost of } \\
\text { fatal } \\
\text { collisions }\end{array}$ & $\begin{array}{l}\text { Cost of injury } \\
\text { collisions }\end{array}$ & $\begin{array}{c}\text { Cost of PDO } \\
\text { collisions }\end{array}$ & $\begin{array}{l}\text { Total } \\
\text { Weighted } \\
\text { Cost }\end{array}$ \\
\hline Total collisions (observed) & 34 & 0.21 & 55.83 & 175.35 & $\begin{array}{l}\$ \\
5,058,282\end{array}$ & $\begin{array}{l}\$ \\
7,481,530\end{array}$ & $\begin{array}{l}\$ \\
1,928,892\end{array}$ & $\begin{array}{l}\$ \\
14,468,703\end{array}$ \\
\hline $\begin{array}{l}\text { Total collisions (PSM counterfactual } \\
\text { would have been } 2.1 \% \text { increase) }\end{array}$ & 34 & 0.21 & 57.02 & 179.08 & $\begin{array}{l}\$ \\
5,165,738\end{array}$ & $\begin{array}{l}\$ \\
7,640,464\end{array}$ & $\begin{array}{l}\$ \\
1,969,868\end{array}$ & $\begin{array}{l}\$ \\
14,776,070\end{array}$ \\
\hline $\begin{array}{l}\text { Difference between (observed - } \\
\text { counterfactual) }\end{array}$ & & 0.00 & -1.19 & -3.73 & $\begin{array}{l}-\$ \\
107,456\end{array}$ & $\begin{array}{l}-\$ \\
158,934\end{array}$ & $\begin{array}{l}-\$ \\
40,977\end{array}$ & $\begin{array}{l}-\$ \\
307,367\end{array}$ \\
\hline Rearend collisions (observed) & 34 & 0.00 & 23.92 & 85.10 & $\begin{array}{c}\$ \\
-\end{array}$ & $\begin{array}{l}\$ \\
3,205,039\end{array}$ & $\begin{array}{l}\$ \\
936,147\end{array}$ & $\begin{array}{l}\$ \\
4,141,186\end{array}$ \\
\hline $\begin{array}{l}\text { Rear-end collisions (PSM } \\
\text { counterfactual would have been } \\
11.9 \% \text { decrease) }\end{array}$ & 34 & 0.00 & 21.08 & 75.00 & $\begin{array}{c}\$ \\
- \\
\end{array}$ & 2824356.08 & 824955.10 & 3649311.17 \\
\hline $\begin{array}{l}\text { Difference between (observed - } \\
\text { counterfactual) }\end{array}$ & & 0.00 & 2.84 & 10.11 & $\begin{array}{c}\$ \\
-\end{array}$ & $\begin{array}{l}\$ \\
380,683 \\
\end{array}$ & $\begin{array}{l} \\
111,192 \\
\end{array}$ & $\begin{array}{l} \\
491,875 \\
\end{array}$ \\
\hline Angle collisions (observed) & 34 & 0.21 & 13.91 & 28.51 & $\begin{array}{l}\$ \\
5,058,282\end{array}$ & $\begin{array}{l}\$ \\
1,863,395\end{array}$ & $\begin{array}{l}\$ \\
313,579\end{array}$ & $\begin{array}{l}\$ \\
7,235,256\end{array}$ \\
\hline $\begin{array}{l}\text { Angle collisions (PSM counterfactual } \\
\text { would have been } 18.9 \% \text { increase) }\end{array}$ & 34 & 0.25 & 16.53 & 33.89 & $\begin{array}{l}\$ \\
6,012,801\end{array}$ & $\begin{array}{l}\$ \\
2,215,025\end{array}$ & $\begin{array}{l}\$ \\
372,752\end{array}$ & $\begin{array}{l}\$ \\
8,600,578\end{array}$ \\
\hline $\begin{array}{l}\text { Difference between (observed - } \\
\text { counterfactual) }\end{array}$ & & -0.04 & -2.62 & -5.38 & $\begin{array}{l}\$ \\
954,518\end{array}$ & $\begin{array}{l}-\$ \\
351,630\end{array}$ & $\begin{array}{l}\$ \\
59,174\end{array}$ & $\begin{array}{l}-\$ \\
1,365,322\end{array}$ \\
\hline
\end{tabular}




\begin{tabular}{|c|c|c|c|c|c|c|c|c|}
\hline $\begin{array}{l}\text { Turningmovement collisions } \\
\text { (observed) }\end{array}$ & 34 & 0.00 & 11.47 & 25.73 & $\begin{array}{c}\$ \\
- \\
\end{array}$ & $\begin{array}{l}\$ \\
1,537,301 \\
\end{array}$ & $\begin{array}{l}\$ \\
282,986 \\
\end{array}$ & $\begin{array}{l}\$ \\
1,820,286\end{array}$ \\
\hline $\begin{array}{l}\text { Turning movement collisions (PSM } \\
\text { counterfactual would have been } \\
20.8 \% \text { increase) }\end{array}$ & 34 & 0.00 & 13.86 & 31.07 & $\begin{array}{c}\$ \\
-\end{array}$ & $\begin{array}{l}\$ \\
1,856,839\end{array}$ & $\begin{array}{l}\$ \\
341,806\end{array}$ & $\begin{array}{l}\$ \\
2,198,645\end{array}$ \\
\hline $\begin{array}{l}\text { Difference between (observed - } \\
\text { counterfactual) }\end{array}$ & & 0.00 & -2.38 & -5.35 & $\begin{array}{c}\$ \\
-\end{array}$ & $\begin{array}{l}-\$ \\
319,538\end{array}$ & $\begin{array}{l}-\$ \\
58,820\end{array}$ & $\begin{array}{l}-\$ \\
378,358 \\
\end{array}$ \\
\hline Sideswipe collisions (observed) & 34 & 0.00 & 1.32 & 25.87 & $\begin{array}{c}\$ \\
-\end{array}$ & $\begin{array}{l}\$ \\
177,022\end{array}$ & $\begin{array}{l}\$ \\
284,515\end{array}$ & $\begin{array}{l}\$ \\
461,538\end{array}$ \\
\hline $\begin{array}{l}\text { Sideswipe collisions (PSM } \\
\text { counterfactual would have been } 6.7 \% \\
\text { decrease) }\end{array}$ & 34 & 0.00 & 1.23 & 24.13 & $\begin{array}{c}\$ \\
-\end{array}$ & $\begin{array}{l}\$ \\
165,161\end{array}$ & $\begin{array}{l}\$ \\
265,451\end{array}$ & $\begin{array}{l}\$ \\
430,611\end{array}$ \\
\hline $\begin{array}{l}\text { Difference between (observed - } \\
\text { counterfactual) }\end{array}$ & & 0.00 & 0.09 & 1.73 & $\begin{array}{c}\$ \\
-\end{array}$ & $\begin{array}{l}\$ \\
11,862\end{array}$ & $\begin{array}{l}\$ \\
19,065\end{array}$ & $\begin{array}{l}\$ \\
30,926\end{array}$ \\
\hline
\end{tabular}




\section{LOW COSTS}

\begin{tabular}{|c|c|c|c|c|c|c|c|c|}
\hline \multirow[b]{2}{*}{ Collision Impact Type } & \multirow[b]{2}{*}{$\begin{array}{l}\text { \# of RLC } \\
\text { sites }\end{array}$} & \multicolumn{3}{|c|}{ Annual number of collisions } & \multicolumn{3}{|c|}{ Annual cost of collisions } & \multirow[b]{2}{*}{$\begin{array}{l}\text { Total } \\
\text { Weighted } \\
\text { Cost }\end{array}$} \\
\hline & & $\begin{array}{c}\text { Annual } \\
\text { number of } \\
\text { Fatal } \\
\text { collisions } \\
\text { after RLC }\end{array}$ & $\begin{array}{c}\text { Annual } \\
\text { number of } \\
\text { Injury } \\
\text { collisions } \\
\text { after RLC }\end{array}$ & $\begin{array}{c}\text { Annual } \\
\text { number of } \\
\text { PDO } \\
\text { collisions } \\
\text { after RLC }\end{array}$ & $\begin{array}{l}\text { Cost of } \\
\text { fatal } \\
\text { collisions }\end{array}$ & $\begin{array}{l}\text { Cost of injury } \\
\text { collisions }\end{array}$ & $\begin{array}{c}\text { Cost of PDO } \\
\text { collisions }\end{array}$ & \\
\hline Total collisions (observed) & 34 & 0.21 & 55.83 & 175.35 & $\begin{array}{l}\$ \\
1,925,276\end{array}$ & $\begin{array}{l}\$, 398,525 \\
\end{array}$ & $\begin{array}{l}\$ \\
1,717,064\end{array}$ & $\begin{array}{l}\$ \\
8,040,866\end{array}$ \\
\hline $\begin{array}{l}\text { Total collisions (PSM counterfactual } \\
\text { would have been } 2.1 \% \text { increase) }\end{array}$ & 34 & 0.21 & 57.02 & 179.08 & $\begin{array}{l}\$ \\
1,966,176 \\
\end{array}$ & $\begin{array}{l}\$ \\
4,491,966 \\
\end{array}$ & $\begin{array}{l}\$ \\
1,753,541\end{array}$ & $\begin{array}{l}\$ \\
8,211,682 \\
\end{array}$ \\
\hline $\begin{array}{l}\text { Difference between (observed - } \\
\text { counterfactual) }\end{array}$ & & 0.00 & -1.19 & -3.73 & $\begin{array}{l}-\$ \\
40,900 \\
\end{array}$ & $\begin{array}{l}-\$ \\
93,440 \\
\end{array}$ & $\begin{array}{l}\$ \\
36,477 \\
\end{array}$ & $\begin{array}{l}-\$ \\
170,817 \\
\end{array}$ \\
\hline Rearend collisions (observed) & 34 & 0.00 & 23.92 & 85.10 & $\begin{array}{c}\$ \\
-\end{array}$ & $\begin{array}{l}\$ \\
1,884,300\end{array}$ & $\begin{array}{l}\$ \\
833,341\end{array}$ & $\begin{array}{l}\$ \\
2,717,641 \\
\end{array}$ \\
\hline $\begin{array}{l}\text { Rear-end collisions (PSM } \\
\text { counterfactual would have been } \\
11.9 \% \text { decrease) }\end{array}$ & 34 & 0.00 & 21.08 & 75.00 & $\begin{array}{c}\$ \\
- \\
\end{array}$ & 1660489.52 & 734360.03 & 2394849.55 \\
\hline $\begin{array}{l}\text { Difference between (observed - } \\
\text { counterfactual) }\end{array}$ & & 0.00 & 2.84 & 10.11 & $\begin{array}{l}\$ \\
-\end{array}$ & $\begin{array}{l}\$ \\
223,810 \\
\end{array}$ & $\begin{array}{l}\$ \\
98,981 \\
\end{array}$ & $\begin{array}{l}\$ \\
\$ 22,791 \\
\end{array}$ \\
\hline Angle collisions (observed) & 34 & 0.21 & 13.91 & 28.51 & $\begin{array}{l}\$ \\
1,925,276\end{array}$ & $\begin{array}{l}\$ \\
1,095,523\end{array}$ & $\begin{array}{l}\$ \\
279,142\end{array}$ & $\begin{array}{l}\$ \\
3,299,941 \\
\end{array}$ \\
\hline $\begin{array}{l}\text { Angle collisions (PSM counterfactual } \\
\text { would have been } 18.9 \% \text { increase) }\end{array}$ & 34 & 0.25 & 16.53 & 33.89 & $\begin{array}{l}\$ \\
2,288,583\end{array}$ & $\begin{array}{l}\$ \\
1,302,253\end{array}$ & $\begin{array}{l}\$ \\
331,817\end{array}$ & $\begin{array}{l}\$ \\
3,922,654\end{array}$ \\
\hline $\begin{array}{l}\text { Difference between (observed - } \\
\text { counterfactual) }\end{array}$ & & -0.04 & -2.62 & -5.38 & $\begin{array}{l}\$ \\
363,307\end{array}$ & $\begin{array}{l}\$ \\
206,730\end{array}$ & $\begin{array}{l}-\$ \\
52,675\end{array}$ & $\begin{array}{l}-\$ \\
622,712\end{array}$ \\
\hline
\end{tabular}




\begin{tabular}{|c|c|c|c|c|c|c|c|c|}
\hline $\begin{array}{l}\begin{array}{l}\text { Turningmovement collisions } \\
\text { (observed) }\end{array} \\
\end{array}$ & 34 & 0.00 & 11.47 & 25.73 & $\begin{array}{l}\$ \\
- \\
\end{array}$ & $\begin{array}{l}\$ \\
903,807 \\
\end{array}$ & $\begin{array}{l}\$ \\
251,909 \\
\end{array}$ & $\begin{array}{l}\$ \\
1,155,715\end{array}$ \\
\hline $\begin{array}{l}\text { Turning movement collisions (PSM } \\
\text { counterfactual would have been } \\
20.8 \% \text { increase) }\end{array}$ & 34 & 0.00 & 13.86 & 31.07 & $\begin{array}{l}\$ \\
-\end{array}$ & $\begin{array}{l}\$ \\
1,091,669\end{array}$ & $\begin{array}{l}\$ \\
304,270\end{array}$ & $\begin{array}{l}\$ \\
1,395,938\end{array}$ \\
\hline $\begin{array}{l}\text { Difference between (observed - } \\
\text { counterfactual) }\end{array}$ & & 0.00 & -2.38 & -5.35 & $\begin{array}{l}\$ \\
- \\
\end{array}$ & $\begin{array}{l}-\$ \\
187,862\end{array}$ & $\begin{array}{l}-\$ \\
52,361\end{array}$ & $\begin{array}{l}-\$ \\
240,223 \\
\end{array}$ \\
\hline Sideswipe collisions (observed) & 34 & 0.00 & 1.32 & 25.87 & $\$$ & $\begin{array}{l}\$ \\
104,075\end{array}$ & $\begin{array}{l}\$ \\
253,270\end{array}$ & $\begin{array}{l}\$ \\
357,345\end{array}$ \\
\hline $\begin{array}{l}\text { Sideswipe collisions (PSM } \\
\text { counterfactual would have been } 6.7 \% \\
\text { decrease) }\end{array}$ & 34 & 0.00 & 1.23 & 24.13 & $\begin{array}{l}\$ \\
- \\
\end{array}$ & $\begin{array}{l}\$ \\
97,101\end{array}$ & $\begin{array}{l}\$ \\
236,299 \\
\end{array}$ & $\begin{array}{l}\$ \\
333,400 \\
\end{array}$ \\
\hline $\begin{array}{l}\text { Difference between (observed - } \\
\text { counterfactual) }\end{array}$ & & 0.00 & 0.09 & 1.73 & $\$$ & $\begin{array}{l}\$ \\
6,974\end{array}$ & $\begin{array}{l}\$ \\
16,971\end{array}$ & $\begin{array}{l}\$ \\
23,945\end{array}$ \\
\hline
\end{tabular}




\section{HIGH RLC}

\begin{tabular}{|c|c|c|c|c|c|c|c|c|}
\hline & & Annual & number of $c$ & llisions & & ual cost of collis & ons & \\
\hline Collision Impact Type & $\begin{array}{l}\text { \# of RLC } \\
\text { sites }\end{array}$ & $\begin{array}{c}\text { Annual } \\
\text { number of } \\
\text { Fatal } \\
\text { collisions } \\
\text { after RLC }\end{array}$ & $\begin{array}{c}\text { Annual } \\
\text { number of } \\
\text { Injury } \\
\text { collisions } \\
\text { after RLC }\end{array}$ & $\begin{array}{c}\text { Annual } \\
\text { number of } \\
\text { PDO } \\
\text { collisions } \\
\text { after RLC }\end{array}$ & $\begin{array}{l}\text { Cost of } \\
\text { fatal } \\
\text { collisions }\end{array}$ & $\begin{array}{l}\text { Cost of injury } \\
\text { collisions }\end{array}$ & $\begin{array}{c}\text { Cost of PDO } \\
\text { collisions }\end{array}$ & $\begin{array}{c}\text { Total } \\
\text { Weighted } \\
\text { Cost }\end{array}$ \\
\hline Total collisions (observed) & 34 & 0.21 & 55.83 & 175.35 & $\begin{array}{l}\$ \\
4,046,626 \\
\end{array}$ & $\begin{array}{l}\$ \\
5,661,396 \\
\end{array}$ & $\begin{array}{l}\$ \\
1,753,538 \\
\end{array}$ & $\begin{array}{l}\$ \\
11,461,560 \\
\end{array}$ \\
\hline Total collisions (counterfactual) & 34 & 0.21 & 57.02 & 179.08 & $\begin{array}{l}\$ \\
4,132,590\end{array}$ & $\begin{array}{l}\$ \\
5,781,665\end{array}$ & $\begin{array}{l}\$ \\
1,790,789 \\
\end{array}$ & $\begin{array}{l}\$ \\
11,705,044 \\
\end{array}$ \\
\hline $\begin{array}{l}\text { Difference between (observed - } \\
\text { counterfactual) }\end{array}$ & & 0.00 & -1.19 & -3.73 & $\begin{array}{l}-\$ \\
85,965\end{array}$ & $\begin{array}{l}\$ \\
120,268\end{array}$ & $\begin{array}{l}-\$ \\
37,251\end{array}$ & $\begin{array}{l}-\$ \\
243,484 \\
\end{array}$ \\
\hline Rearend collisions (observed) & 34 & 0.00 & 23.92 & 85.10 & $\begin{array}{c}\$ \\
-\end{array}$ & $\begin{array}{l}\$ \\
2,425,306\end{array}$ & $\begin{array}{l}\$ \\
851,043\end{array}$ & $\begin{array}{l}\$ \\
3,276,348\end{array}$ \\
\hline Rear-end collisions (counterfactual) & 34 & 0.00 & 23.49 & 83.56 & $\$$ & 2381436.95 & 835649.40 & 3217086.35 \\
\hline $\begin{array}{l}\text { Difference between (observed - } \\
\text { counterfactual) }\end{array}$ & & 0.00 & 0.43 & 1.54 & $\$$ & $\begin{array}{l}\$ \\
43,869\end{array}$ & $\begin{array}{l}\$ \\
15,394 \\
\end{array}$ & $\begin{array}{l}\$ \\
59,262 \\
\end{array}$ \\
\hline Angle collisions (observed) & 34 & 0.21 & 13.91 & 28.51 & $\begin{array}{l}\$ \\
4,046,626\end{array}$ & $\begin{array}{l}\$ \\
1,410,061\end{array}$ & $\begin{array}{l}\$ \\
285,072\end{array}$ & $\begin{array}{l}\$ \\
5,741,759\end{array}$ \\
\hline Angle collisions (counterfactual) & 34 & 0.32 & 21.39 & 43.84 & $\begin{array}{l}\$ \\
6,223,710 \\
\end{array}$ & $\begin{array}{l}\$ \\
2,168,674\end{array}$ & $\begin{array}{l}\$ \\
438,440\end{array}$ & $\begin{array}{l}\$ \\
8,830,825\end{array}$ \\
\hline $\begin{array}{l}\text { Difference between (observed - } \\
\text { counterfactual) }\end{array}$ & & -0.11 & -7.48 & -15.34 & $\begin{array}{l}\$ \\
2,177,085\end{array}$ & $\begin{array}{l}-\$ \\
758,613\end{array}$ & $\begin{array}{l}-\$ \\
153,369\end{array}$ & $\begin{array}{l}-\$ \\
3,089,066\end{array}$ \\
\hline
\end{tabular}




\begin{tabular}{|c|c|c|c|c|c|c|c|c|}
\hline $\begin{array}{l}\text { Turningmovement collisions } \\
\text { (observed) }\end{array}$ & 34 & 0.00 & 11.47 & 25.73 & $\begin{array}{c}\$ \\
-\end{array}$ & $\begin{array}{l}\$ \\
1,163,301\end{array}$ & $\begin{array}{l}\$ \\
257,260\end{array}$ & $\begin{array}{l}\$ \\
1,420,560\end{array}$ \\
\hline $\begin{array}{l}\text { Turning movement collisions } \\
\text { (counterfactua) }\end{array}$ & 34 & 0.00 & 14.02 & 31.44 & $\begin{array}{l}\$ \\
-\end{array}$ & $\begin{array}{l}\$ \\
1,421,553\end{array}$ & $\begin{array}{l}\$ \\
314,371\end{array}$ & $\begin{array}{l}\$ \\
1,735,925\end{array}$ \\
\hline $\begin{array}{l}\text { Difference between (observed - } \\
\text { counterfactual) }\end{array}$ & & 0.00 & -2.55 & -5.71 & $\begin{array}{l}\$ \\
-\end{array}$ & $\begin{array}{l}-\$ \\
258,253\end{array}$ & $\begin{array}{l}-\$ \\
57,112\end{array}$ & $\begin{array}{l}-\$ \\
315,364\end{array}$ \\
\hline Sideswipe collisions (observed) & 34 & 0.00 & 1.32 & 25.87 & $\begin{array}{l}\$ \\
-\end{array}$ & $\begin{array}{l}\$ \\
133,956\end{array}$ & $\begin{array}{l}\$ \\
258,650\end{array}$ & $\begin{array}{l}\$ \\
392,606\end{array}$ \\
\hline Sideswipe collisions (counterfactual) & 34 & 0.00 & 1.23 & 24.13 & $\begin{array}{l}\$ \\
-\end{array}$ & $\begin{array}{l}\$ \\
124,980\end{array}$ & $\begin{array}{l}\$ \\
241,319\end{array}$ & $\begin{array}{l}\$ \\
366,299\end{array}$ \\
\hline $\begin{array}{l}\text { Difference between (observed - } \\
\text { counterfactual) }\end{array}$ & & 0.00 & 0.09 & 1.73 & $\$$ & $\begin{array}{l}\$ \\
8,976\end{array}$ & $\begin{array}{l}\$ \\
17,331\end{array}$ & $\begin{array}{l}\$ \\
26,307\end{array}$ \\
\hline
\end{tabular}




\section{LOW RLC}

\begin{tabular}{|c|c|c|c|c|c|c|c|c|}
\hline & & \multicolumn{3}{|c|}{ Annual number of collisions } & \multicolumn{3}{|c|}{ Annual cost of collisions } & \multirow[b]{2}{*}{$\begin{array}{c}\text { Total } \\
\text { Weighted } \\
\text { Cost }\end{array}$} \\
\hline Collision Impact Type & $\begin{array}{l}\text { \# of RLC } \\
\text { sites }\end{array}$ & $\begin{array}{c}\text { Annual } \\
\text { number of } \\
\text { Fatal } \\
\text { collisions } \\
\text { after RLC }\end{array}$ & $\begin{array}{c}\text { Annual } \\
\text { number of } \\
\text { Injury } \\
\text { collisions } \\
\text { after RLC }\end{array}$ & $\begin{array}{c}\text { Annual } \\
\text { number of } \\
\text { PDO } \\
\text { collisions } \\
\text { after RLC }\end{array}$ & $\begin{array}{c}\text { Cost of } \\
\text { fatal } \\
\text { collisions }\end{array}$ & $\begin{array}{l}\text { Cost of injury } \\
\text { collisions }\end{array}$ & $\begin{array}{l}\text { Cost of PDO } \\
\text { collisions }\end{array}$ & \\
\hline Total collisions (observed) & 34 & 0.21 & 55.83 & 175.35 & $\begin{array}{l}\$ \\
4,046,626 \\
\end{array}$ & $\begin{array}{l}\$ \\
5,661,396 \\
\end{array}$ & $\begin{array}{l}\$ \\
1,753,538 \\
\end{array}$ & $\begin{array}{l}\$ \\
11,461,560\end{array}$ \\
\hline Total collisions (counterfactual) & 34 & 0.20 & 52.71 & 165.55 & $\begin{array}{l}\$ \\
3,820,419\end{array}$ & $\begin{array}{l}\$ \\
5,344,924\end{array}$ & $\begin{array}{l}\$ \\
1,655,515\end{array}$ & $\begin{array}{l}\$ \\
10,820,859\end{array}$ \\
\hline $\begin{array}{l}\text { Difference between (observed - } \\
\text { counterfactual) }\end{array}$ & & 0.01 & 3.12 & 9.80 & $\begin{array}{l}\$ \\
226,206\end{array}$ & $\begin{array}{l}\$ \\
316,472\end{array}$ & $\begin{array}{l}\$ \\
98,023\end{array}$ & $\begin{array}{l}\$ \\
640,701 \\
\end{array}$ \\
\hline Rearend collisions (observed) & 34 & 0.00 & 23.92 & 85.10 & $\begin{array}{l}\$ \\
- \\
\end{array}$ & $\begin{array}{l}\$ \\
2,425,306 \\
\end{array}$ & $\begin{array}{l}\$ \\
851,043 \\
\end{array}$ & $\begin{array}{l}\$ \\
3,276,348 \\
\end{array}$ \\
\hline Rear-end collisions (counterfactual) & 34 & 0.00 & 18.23 & 64.85 & $\begin{array}{c}\$ \\
-\end{array}$ & 1848082.81 & 648494.72 & 2496577.53 \\
\hline $\begin{array}{l}\text { Difference between (observed - } \\
\text { counterfactual) }\end{array}$ & & 0.00 & 5.69 & 20.25 & $\$$ & $\begin{array}{l}\$ \\
577,223 \\
\end{array}$ & $\begin{array}{l}\$ \\
202,548\end{array}$ & $\begin{array}{l}\$ \\
779,771 \\
\end{array}$ \\
\hline Angle collisions (observed) & 34 & 0.21 & 13.91 & 28.51 & $\begin{array}{l}\$ \\
4,046,626\end{array}$ & $\begin{array}{l}\$ \\
1,410,061\end{array}$ & $\begin{array}{l}\$ \\
285,072\end{array}$ & $\begin{array}{l}\$ \\
5,741,759\end{array}$ \\
\hline Angle collisions (counterfactual) & 34 & 0.23 & 15.53 & 31.84 & $\begin{array}{l}\$ \\
4,520,081\end{array}$ & $\begin{array}{l}\$ \\
1,575,039\end{array}$ & $\begin{array}{l}\$ \\
318,425\end{array}$ & $\begin{array}{l}\$ \\
6,413,544 \\
\end{array}$ \\
\hline $\begin{array}{l}\text { Difference between (observed - } \\
\text { counterfactual) }\end{array}$ & & -0.02 & -1.63 & -3.34 & $\begin{array}{l}\$ \\
473,455\end{array}$ & $\begin{array}{l}\$ \\
164,977\end{array}$ & $\begin{array}{l}-\$ \\
33,353\end{array}$ & $\begin{array}{l}-\$ \\
671,786\end{array}$ \\
\hline
\end{tabular}




\begin{tabular}{|c|c|c|c|c|c|c|c|c|}
\hline $\begin{array}{l}\text { Turningmovement collisions } \\
\text { (observed) }\end{array}$ & 34 & 0.00 & 11.47 & 25.73 & $\begin{array}{l}\$ \\
- \\
\end{array}$ & $\begin{array}{l}\$ \\
1,163,301 \\
\end{array}$ & $\begin{array}{l}\$ \\
257,260\end{array}$ & $\begin{array}{l}\$ \\
1,420,560 \\
\end{array}$ \\
\hline $\begin{array}{l}\text { Turning movement collisions } \\
\text { (counterfactual) }\end{array}$ & 34 & 0.00 & 12.47 & 27.96 & $\begin{array}{c}\$ \\
-\end{array}$ & $\begin{array}{l}\$ \\
1,264,508\end{array}$ & $\begin{array}{l}\$ \\
279,641\end{array}$ & $\begin{array}{l}\$ \\
1,544,149\end{array}$ \\
\hline $\begin{array}{l}\text { Difference between (observed - } \\
\text { counterfactual) }\end{array}$ & & 0.00 & -1.00 & -2.24 & $\begin{array}{l}\$ \\
-\end{array}$ & $\begin{array}{l}-\$ \\
101,207\end{array}$ & $\begin{array}{l}-\$ \\
22,382\end{array}$ & $\begin{array}{l}-\$ \\
123,589\end{array}$ \\
\hline Sideswipe collisions (observed) & 34 & 0.00 & 1.32 & 25.87 & $\begin{array}{l}\$ \\
- \\
\end{array}$ & $\begin{array}{l}\$ \\
133,956 \\
\end{array}$ & $\begin{array}{l}\$ \\
258,650\end{array}$ & $\begin{array}{l}\$ \\
392,606 \\
\end{array}$ \\
\hline Sideswipe collisions (counterfactual) & 34 & 0.00 & 0.87 & 16.99 & $\begin{array}{l}\$ \\
-\end{array}$ & $\begin{array}{l}\$ \\
88,009\end{array}$ & $\begin{array}{l}\$ \\
169,933\end{array}$ & $\begin{array}{l}\$ \\
257,942\end{array}$ \\
\hline $\begin{array}{l}\text { Difference between (observed - } \\
\text { counterfactual) }\end{array}$ & & 0.00 & 0.45 & 8.87 & $\$$ & $\begin{array}{l}\$ \\
45,947\end{array}$ & $\begin{array}{l}\$ \\
88,717\end{array}$ & $\begin{array}{l}\text { \$ } \\
134,664\end{array}$ \\
\hline
\end{tabular}

